\title{
Mapping and Localisation for Mobile Robots
}

\author{
by \\ Tun Yong Yang \\ A thesis submitted to \\ The Faculty of Graduate Studies and Research \\ in partial fulfilment \\ of the requirements for the degree of \\ Master of Applied Science in Electrical Engineering \\ Ottawa-Carleton Institute of Electrical and Computer Engineering \\ Department of Systems and Computer Engineering \\ Carleton University \\ Ottawa, Ontario \\ August 6, 2004 \\ (C)Copyright \\ 2004 , Tun Yong Yang
}




$\begin{array}{ll}\begin{array}{l}\text { Library and } \\ \text { Archives Canada }\end{array} & \begin{array}{l}\text { Bibliothèque et } \\ \text { Archives Canada }\end{array} \\ \begin{array}{l}\text { Published Heritage } \\ \text { Branch }\end{array} & \begin{array}{l}\text { Direction du } \\ \text { Patrimoine de l'édition }\end{array} \\ \begin{array}{l}\text { 395 Wellington Street } \\ \text { Ottawa ON K1A 0N4 }\end{array} & \begin{array}{l}\text { 395, rue Wellington } \\ \text { Ottawa ON K1A ON4 } \\ \text { Canada }\end{array} \\ \end{array}$

Your file Votre référence ISBN: 0-612-97446-4

Ourfile Notre référence

ISBN: 0-612-97446-4

NOTICE:

The author has granted a nonexclusive license allowing Library and Archives Canada to reproduce, publish, archive, preserve, conserve, communicate to the public by telecommunication or on the Internet, loan, distribute and sell theses worldwide, for commercial or noncommercial purposes, in microform, paper, electronic and/or any other formats.

The author retains copyright ownership and moral rights in this thesis. Neither the thesis nor substantial extracts from it may be printed or otherwise reproduced without the author's permission.
AVIS:

L'auteur a accordé une licence non exclusive permettant à la Bibliothèque et Archives Canada de reproduire, publier, archiver, sauvegarder, conserver, transmettre au public par télécommunication ou par l'Internet, prêter, distribuer et vendre des thèses partout dans le monde, à des fins commerciales ou autres, sur support microforme, papier, électronique et/ou autres formats.

L'auteur conserve la propriété du droit d'auteur et des droits moraux qui protège cette thèse. $\mathrm{Ni}$ la thèse ni des extraits substantiels de celle-ci ne doivent être imprimés ou autrement reproduits sans son autorisation.
In compliance with the Canadian

Privacy Act some supporting forms may have been removed from this thesis.

While these forms may be included in the document page count, their removal does not represent any loss of content from the thesis.
Conformément à la loi canadienne sur la protection de la vie privée, quelques formulaires secondaires ont été enlevés de cette thèse.

Bien que ces formulaires aient inclus dans la pagination, il n'y aura aucun contenu manquant. 
pagIRATION ERROR.

IETI COLPLEIR.
ERBEUR DE PAGIRATION.

IE TEXIE EST CONPLET. 


\section{Abstract}

Autonomous mobile robots must act intelligently without external control by definition, and require fundamental capabilities such as the awareness of its environment, and of its location within the environment. These two problems are known respectively as mapping and localisation. Different solutions to the problems are surveyed, and selected techniques are examined using a software simulated robot equipped with laser range sensors. An occupancy grid mapping technique using Dempster-Shafer ev-

idential theory is implemented, and the validity and sensitivity to parameter changes examined using simulation results. Localisation techniques based on particle filters are also implemented, and simulation results are contrasted using different criteria. The localisation techniques are the Monte Carlo localisation (MCL) method using a weighted bootstrap particle filter, the clustered particle filtering MCL (CPF-MCL) method, and the uniform MCL (U-MCL) method. An extension to the CPF-MCL and U-MCL methods is proposed to create the uniform CPF-MCL (UCPF-MCL) method, and also evaluated. 


\section{Acknowledgements}

After years of learning and higher learning, a research thesis is a wonderful piece of work. It requires dilligence, ingenuity, and plain simple hard work. The thesis process also reveals the enlightening effect of knowledge and research that cannot be copied from others, and the task eternal. This thesis builds on top of research performed by others, but is a product of myself alone with the influence and guidance from the many years. For this, I wish to acknowledge my family, for giving me the life I have gone through, good times and bad, for I would be a different person without them; Carleton University, and all the faculty and students, for though they may not know me, I have learned a great deal from them; Prof. Majumdar, Prof. L. Briand, and C. Chean for their support; Prof. V. Aitken, for the support and guidance in the numerous aspects of this thesis; the National Science and Engineering Research Council, without whose financial support, this thesis would not be possible; and finally, W. W. Chean, whose entrance in my life, is a blessing never to be forgotten. 


\section{Contents}

Contents $v$

List of Tables $\quad$ x

List of Figures $\quad$ Xv

1 Introduction 1

1.1 Robot Architecture . . . . . . . . . . . . . . . . . 3

1.2 Contemporary Robot Mapping and Localisation . . . . . . . . . . . . 7

1.3 Thesis Statement . . . . . . . . . . . . . . . . 9

1.4 Thesis Overview . . . . . . . . . . . . . . . . 11

2 Background $\quad 13$

2.1 Issues in Mapping and Localisation . . . . . . . . . . . . . . . 15

2.2 Mapping . . . . . . . . . . . . . . . . . . 19

2.2.1 Grid Based Map . . . . . . . . . . . . . . 20

2.2.2 Topological Based Map . . . . . . . . . . . . . . 26

2.2 .3 Combined Maps . . . . . . . . . . . . . . . . . 32

2.3 Localisation . . . . . . . . . . . . . . . . . 33 
2.3.1 Kalman Filter . . . . . . . . . . . . . 36

2.3.2 Markov Localisation . . . . . . . . . . . . . . . 38

2.3.3 Monte Carlo Localisation . . . . . . . . . . . . . . . 43

2.3.4 Alternative Localisation . . . . . . . . . . . . . 46

2.4 Simultaneous Localisation and Mapping . . . . . . . . . . . . . . 47

2.4.1 Kalman Filter Solution . . . . . . . . . . . . . . . 48

2.4.2 Expectation Maximization Solution . . . . . . . . . . . 50

2.4.3 Incremental Solution . . . . . . . . . . . . . 51

2.5 Chapter Summary . . . . . . . . . . . . . . . 53

3 Theoretical Mapping and Localisation $\quad 54$

3.1 Mapping ........................... 56

3.1.1 Meaning of Probabilities . . . . . . . . . . . . . . 56

3.1 .2 Bayesian Theory ........................ 58

3.1.3 Bayesian Environment Mapping . . . . . . . . . . . . . 61

3.1.4 Dempster-Shafer Theory of Evidence . . . . . . . . . . 63

3.1.5 Evidential Environment Mapping . . . . . . . . . . 72

3.1.6 Brief Comparison of Bayesian and Evidential Mapping . . . . 74

3.2 Localisation . . . . . . . . . . . . . . . . . 79

3.2.1 Ideal Monte Carlo Sampling . . . . . . . . . . . . . . . 80

3.2.2 Importance Sampling . . . . . . . . . . . . . . 83

3.2.3 Sequential Importance Sampling . . . . . . . . . . . . . . 87

3.2.4 Monte Carlo Localisation . . . . . . . . . . . . . . . 90

3.3 Chapter Summary . . . . . . . . . . . . . 100 
4 Simulator Implementation

4.1 Component Overview . . . . . . . . . . . . . 106

4.2 Implementation Tools . . . . . . . . . . . . . . . . 107

4.3 General Component Implementation . . . . . . . . . . . . . . 108

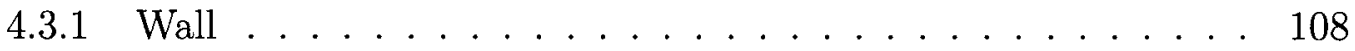

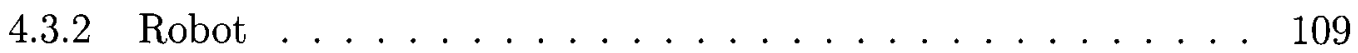

4.3 .3 Driving System . . . . . . . . . . . . . . . . 110

4.3 .4 Sensor System . . . . . . . . . . . . . . 111

4.3.5 Mapping System . . . . . . . . . . . . . 113

4.3 .6 Localisation System . . . . . . . . . . . . . . 113

4.3 .7 Planning System . . . . . . . . . . . . . 113

4.4 Mapping Component Implementation . . . . . . . . . . . . . . 114

4.4 Laser Sensor . . . . . . . . . . . . . . . . . . 114

4.4 .2 EvidentialMapping .................... 115

4.5 Localisation Component Implementation . . . . . . . . . . . . . . . . 117

4.5.1 Odometry Sensor . . . . . . . . . . . . . 117

4.5 .2 Laser Sensor . . . . . . . . . . . . . . . . . . . . 119

4.5.3 Monte Carlo Localisation . . . . . . . . . . . . . . . . 121

4.5.4 Clustered Particle Filtering MCL . . . . . . . . . . . . . 124

4.5 .5 Uniform MCL . . . . . . . . . . . . . 126

4.5.6 Uniform Clustered Particle Filtering MCL . . . . . . . . . . 127

4.6 Chapter Summary . . . . . . . . . . . . . . . 128

5 Simulation Results $\quad 129$

5.1 Simulation Settings . . . . . . . . . . . . . . . . 129 
5.1.1 Robot Configuration ................ 129

5.1 .2 Test Environments . . . . . . . . . . . . . . 131

5.2 Mapping . . . . . . . . . . . . . . . . . 132

5.2.1 Symmetric Map Test Cases . . . . . . . . . . . . . . 132

5.2 .2 Office Map Test Cases . . . . . . . . . . . . . . . . . 145

5.2 .3 Discussion ........................ 151

5.3 Localisation . . . . . . . . . . . . . . . . . . . 152

5.3 .1 Symmetric Map Test Cases . . . . . . . . . . . . 153

5.3 .2 Office Map Test Cases . . . . . . . . . . . . . . . . . 162

5.3 .3 Discussion . . . . . . . . . . . . . . . 172

5.4 Chapter Summary . . . . . . . . . . . . . . . 177

6 Conclusions and Future Research $\quad 179$

6.1 Further Work . . . . . . . . . . . . . . 181

6.1 .1 Evidential Mapping Discussion . . . . . . . . . . . . 182

6.1 .2 MCL Discussion . . . . . . . . . . . . . . . . 182

6.1.3 CPF-MCL Discussion and Improvements . . . . . . . . . . . . 183

6.1.4 UCPF-MCL Discussion and Improvements . . . . . . . . . . . 184

6.2 Future Research . . . . . . . . . . . . . . . . . 184

$\begin{array}{ll}\text { References } & 186\end{array}$

$\begin{array}{ll}\text { Appendix A: Simulator Software } & 202\end{array}$

A.1 Graphical Interface . . . . . . . . . . . . . 204

A.2 Configuration .................... 207

A.2.1 Configuration File . . . . . . . . . . . . . . . 208

viii 
Appendix B: Simulation Results

B.1 Symmetric Map Results . . . . . . . . . . . . . . . . . . . . . 214

B.1.1 MCL . . . . . . . . . . . . . . . . . . 214

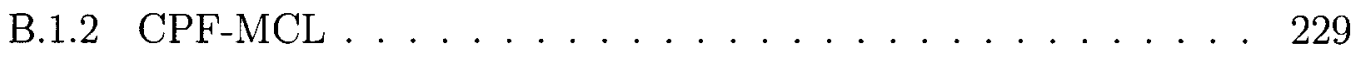

B.1.3 UCPF-MCL . . . . . . . . . . . . . . . . . . . . . 234

B.2 Office Map Results . . . . . . . . . . . . . . . . . . . . . 239

B.2.1 MCL . . . . . . . . . . . . . . . . . . . . . . . . 239

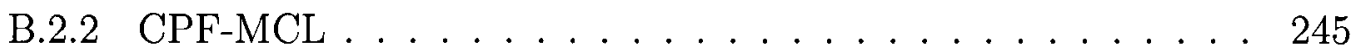

B.2.3 UCPF-MCL . . . . . . . . . . . . . . . . . . . . . . . 251 


\section{List of Tables}

2.1 Advantages and disadvantages of grid based and topological map rep-

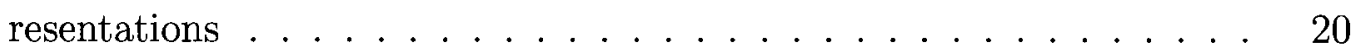

3.1 Dempster-Shafer Example: Summary of Technicians' Opinions . . . . 69

3.2 Dempster-Shafer Example: Table of Calculations . . . . . . . . . 69

3.3 Dempster-Shafer Example: Table of Results for Disjunctive Data In-

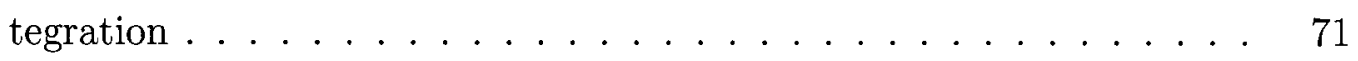

3.4 Dempster-Shafer Example: Table of Results for Trade Off Data Inte-

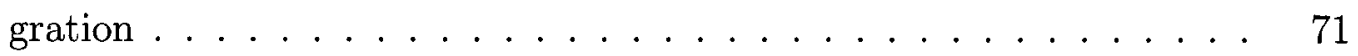

3.5 Results for Sensor Data Merge Using Bayesian and Dempster-Shafer

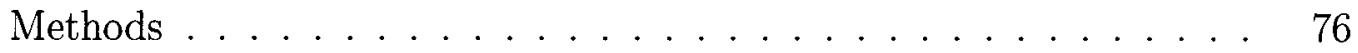

3.6 Results for Data Merge Using Revised Dempster-Shafer Methods . . . 78

3.7 Results for Data Merge Using Revised Dempster-Shafer Methods (Case

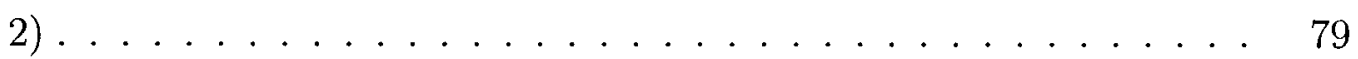

3.8 Summary of Monte Carlo Approximations to Simple Integral . . . . . 83

3.9 Summary of Importance Sampling Approximations to Simple Integral 85

3.10 Weighted Bootstrap Filter Algorithm for Use in MCL . . . . . . . . 93

3.11 Algorithm for a Clustered Particle Filter . . . . . . . . . . . . . 97 
3.12 Algorithm for a Uniform MCL Particle Filter . . . . . . . . . . . . 99

3.13 Algorithm for a Uniform Clustered Particle Filter . . . . . . . . . . . 100

5.1 Mapping Test Cases for Varying Parameters of Laser Sensor . . . . . 133

5.2 Localisation Test Cases and Corresponding Results for Varying Pa-

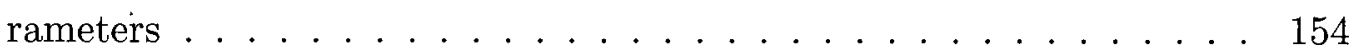

5.3 Summarised MCL1 Symmetric Map Test Case Results . . . . . . . 160

5.4 Summarised MCL2 Symmetric Map Test Case Results . . . . . . . . 160

5.5 Summarised MCL3 Symmetric Map Test Case Results . . . . . . . 161

5.6 Summarised CPF Symmetric Map Test Case Results . . . . . . . . . 161

5.7 Summarised UCPF Symmetric Map Test Case Results . . . . . . . . 162

5.8 Localisation Office Map Test Cases for Varying Parameters . . . . . . 163

5.9 Summarised Office Map Single Run Test Case Results . . . . . . . . . 166

5.10 Test Case Settings for Repeated Runs . . . . . . . . . . . . . . . 169

5.11 Summarised Office Map Test Case R1-1 Results . . . . . . . . . . . . 171

5.12 Summarised Office Map Test Case R1-2 Results . . . . . . . . . . . 171

5.13 Summarised Office Map Test Case R2-1 Results . . . . . . . . . . . . 172

5.14 Summarised Office Map Test Case R2-2 Results . . . . . . . . . . . 172

5.15 Summarised Office Map Test Case R3-1 Results . . . . . . . . . . . 172

5.16 Summarised Office Map Test Case R3-2 Results . . . . . . . . . . . . 173

B-1 Error for Localisation Symmetric Map (MCL) Case 1.1 . . . . . . 215

B-2 Error for Localisation Symmetric Map (MCL) Case 1.2 . . . . . . . 215

B-3 Error for Localisation Symmetric Map (MCL) Case 1.3 . . . . . . . 216

B-4 Error for Localisation Symmetric Map (MCL) Case 1.4 . . . . . . . . 216

B-5 Error for Localisation Symmetric Map (MCL) Case 1.5 . . . . . . . 217 
B-6 Error for Localisation Symmetric Map (MCL) Case 1.6 . . . . . . . . 217

B-7 Error for Localisation Symmetric Map (MCL) Case 1.7 . . . . . . . . 218

B-8 Error for Localisation Symmetric Map (MCL) Case 1.8 . . . . . . . 218

B-9 Error for Localisation Symmetric Map (MCL) Case 1.9 . . . . . . . . 219

B-10 Error for Localisation Symmetric Map (MCL) Case 2.1 . . . . . . . . 219

B-11 Error for Localisation Symmetric Map (MCL) Case 2.2 . . . . . . . . 220

B-12 Error for Localisation Symmetric Map (MCL) Case 2.3 . . . . . . . . 220

B-13 Error for Localisation Symmetric Map (MCL) Case 2.4 . . . . . . . . 221

B-14 Error for Localisation Symmetric Map (MCL) Case 2.5 . . . . . . . 221

B-15 Error for Localisation Symmetric Map (MCL) Case 2.6 . . . . . . . . 222

B-16 Error for Localisation Symmetric Map (MCL) Case 2.7 . . . . . . . . 222

B-17 Error for Localisation Symmetric Map (MCL) Case 2.8 . . . . . . . 223

B-18 Error for Localisation Symmetric Map (MCL) Case 2.9 . . . . . . . . 223

B-19 Error for Localisation Symmetric Map (MCL) Case 3.1 . . . . . . . . 224

B-20 Error for Localisation Symmetric Map (MCL) Case 3.2 . . . . . . . . 224

B-21 Error for Localisation Symmetric Map (MCL) Case 3.3 . . . . . . . 225

B-22 Error for Localisation Symmetric Map (MCL) Case 3.4 . . . . . . . 225

B-23 Error for Localisation Symmetric Map (MCL) Case 3.5 . . . . . . . . 226

B-24 Error for Localisation Symmetric Map (MCL) Case 3.6 . . . . . . . 226

B-25 Error for Localisation Symmetric Map (MCL) Case 3.7 . . . . . . . 227

B-26 Error for Localisation Symmetric Map (MCL) Case 3.8 . . . . . . 227

B-27 Error for Localisation Symmetric Map (MCL) Case 3.9 . . . . . . . . 228

B-28 Error for Localisation Symmetric Map (CPF-MCL) Case 1 . . . . . . 229

B-29 Error for Localisation Symmetric Map (CPF-MCL) Case 2 . . . . . . 229

B-30 Error for Localisation Symmetric Map (CPF-MCL) Case 3 . . . . . . 230 
B-31 Error for Localisation Symmetric Map (CPF-MCL) Case 4 . . . . . . 230

B-32 Error for Localisation Symmetric Map (CPF-MCL) Case 5 . . . . . 231

B-33 Error for Localisation Symmetric Map (CPF-MCL) Case 6 . . . . . . 231

B-34 Error for Localisation Symmetric Map (CPF-MCL) Case 7 . . . . . 232

B-35 Error for Localisation Symmetric Map (CPF-MCL) Case 8 . . . . . . 232

B-36 Error for Localisation Symmetric Map (CPF-MCL) Case 9 . . . . . 233

B-37 Error for Localisation Symmetric Map (UCPF-MCL) Case 1 . . . . . 234

B-38 Error for Localisation Symmetric Map (UCPF-MCL) Case 2 . . . . . 234

B-39 Error for Localisation Symmetric Map (UCPF-MCL) Case 3 . . . . . 235

B-40 Error for Localisation Symmetric Map (UCPF-MCL) Case 4 . . . . . 235

B-41 Error for Localisation Symmetric Map (UCPF-MCL) Case 5 . . . . . 236

B-42 Error for Localisation Symmetric Map (UCPF-MCL) Case 6 . . . . . 236

B-43 Error for Localisation Symmetric Map (UCPF-MCL) Case 7 . . . . . 237

B-44 Error for Localisation Symmetric Map (UCPF-MCL) Case 8 . . . . 237

B-45 Error for Localisation Symmetric Map (UCPF-MCL) Case 9 . . . . 238

B-46 Error for Localisation Office Map (MCL) Case 1 . . . . . . . . . . 239

B-47 Error for Localisation Office Map (MCL) Case $2 \ldots$. . . . . . . . . 240

B-48 Error for Localisation Office Map (MCL) Case 3 . . . . . . . . . . 241

B-49 Error for Localisation Office Map (MCL) Case 4 . . . . . . . . . . . . 242

B-50 Error for Localisation Office Map (MCL) Case 5 . . . . . . . . . 243

B-51 Error for Localisation Office Map (MCL) Case 6 . . . . . . . . . . . 244

B-52 Error for Localisation Office Map (CPF-MCL) Case 1 . . . . . . . 245

B-53 Error for Localisation Office Map (CPF-MCL) Case 2 . . . . . . . . . 246

B-54 Error for Localisation Office Map (CPF-MCL) Case 3 . . . . . . . . . 247

B-55 Error for Localisation Office Map (CPF-MCL) Case 4 . . . . . . . . . 248 
B-56 Error for Localisation Office Map (CPF-MCL) Case 5 . . . . . . . . 249

B-57 Error for Localisation Office Map (CPF-MCL) Case 6 . . . . . . . 250

B-58 Error for Localisation Office Map (UCPF-MCL) Case 1 . . . . . . 251

B-59 Error for Localisation Office Map (UCPF-MCL) Case $2 \ldots \ldots$. . . 252

B-60 Error for Localisation Office Map (UCPF-MCL) Case 3 . . . . . . . 253

B-61 Error for Localisation Office Map (UCPF-MCL) Case 4 . . . . . . . 254

B-62 Error for Localisation Office Map (UCPF-MCL) Case $5 \ldots \ldots . .255$

B-63 Error for Localisation Office Map (UCPF-MCL) Case $6 \ldots \ldots$. . . . 256 


\section{List of Figures}

1.1 Traditional Robot Architecture . . . . . . . . . . . . . 3

1.2 Environments Used in Mapping and Localisation Experiments . . . . 11

1.2a Symmetric Environment . . . . . . . . . . . . . . . . 11

$1.2 \mathrm{~b}$ Office Environment . . . . . . . . . . . . . . . . . 11

2.1 Module Interaction . . . . . . . . . . . . . . . . . . . . . . . 15

2.1a Inaccurate Model of Mobile Robot . . . . . . . . . . . 15

2.1b Possible Model of a Mapping Mobile Robot . . . . . . . . . . 15

2.1c Possible Model of a Localising Mobile Robot . . . . . . . . . 15

2.1d Possible Model of a SLAM Mobile Robot . . . . . . . . . . 15

2.2 Maps Created Using Dead Reckoning Without Accounting for Motion Error . . . . . . . . . . . . . . . . . . 16

2.2a Map Created After Small Amounts of Motion . . . . . . . . 16

2.2b Map Created After First Turning Motion . . . . . . . . . . 16

2.2c Map Created After Full Robot Motion . . . . . . . . . . 16

2.2d Actual Environment After Robot Motion . . . . . . . . . . 16

2.3 Low resolution map with large grid cell size . . . . . . . . . . . 23

2.3a Environment with grid cell overlay . . . . . . . . . . . . 23 
2.3b Possible map occupancy with path to destination . . . . . 23

2.3c Possible map occupancy without path to destination . . . . . 23

2.3d Higher resolution map with collision free path to destination . . 23

2.4 Multiresolution map using quadtree representation from [24] . . . . 24

2.5 Examples of maps created with noisy sensors and ideal motion . . . . 24

2.5a Simple environment with grid overlay . . . . . . . . . . . 24

2.5b Possible map created with noisy range sensors . . . . . . . . 24

2.6 Examples of maps created with ideal sensors and noisy motion . . . . 25

2.6a Possible states after uncertain rightwards motion $\ldots \ldots \ldots 25$

2.6b Corresponding maps created after motion . . . . . . . 25

2.6c Possible map state after combination . . . . . . . . 25

2.7 Environment Division Using Landmark Beacons . . . . . . . . . . 30

2.7a Environment with 3 beacons, different topological areas distinguished by shading . . . . . . . . . . . . . 30

2.7b Environment with 1 beacon occluded . . . . . . . . . . 30

2.8 Environment Division Using Area Configuration . . . . . . . . . . . 31

2.8a Environment division using area shape . . . . . . . . . . 31

2.8b Environment with indistinct areas . . . . . . . . . . . . . 31

2.8c Asymmetrical environment with robot entering from bottom . . 31

2.8d Asymmetrical environment with robot entering from top . . . . 31

2.9 Topological Markov Model for Markov Localisation . . . . . . . . . 42

2.9a Model of a sewage network [57] . . . . . . . . . . . 42

$2.9 \mathrm{~b}$ Model of a corridor junction [119] . . . . . . . . . . 42

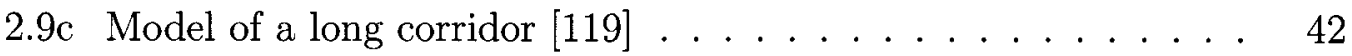

2.10 Symmetric environment illustrating importance of CPF-MCL . . . . 46 
3.1 Example of an Average Mobile Robot . . . . . . . . . . . . . 102

3.1a Blueprint of Realistic Robot . . . . . . . . . . . . . . 102

3.1b Picture of Realistic Robot . . . . . . . . . . . . . . . . 102

3.2 Effect of Sampling Probability Distribution . . . . . . . . . . . 103

3.2a Gaussian Distribution ... . . . . . . . . . . 103

3.2b 100 Sample Approximation (0.5 bin size) . . . . . . . . . . 103

3.2c 1000 Sample Approximation (0.5 bin size) . . . . . . . . . 103

3.2d 10000 Sample Approximation (0.5 bin size) . . . . . . . . . . 103

3.3 Initial Seed Distributions for Localisation . . . . . . . . . . . . . . . . 104

3.3a Uniform Distribution Seed for Localisation . . . . . . . . . . . . 104

3.3b Gaussian Distribution Seed for Localisation . . . . . . . . . . 104

4.1 Simulator Component Diagram . . . . . . . . . . . 106

4.2 Laser Range Measurement Scenario . . . . . . . . . . . . . . . . . 113

4.3 Illustration of Laser Sensor Model for Mapping . . . . . . . . . . . . . 116

4.4 Odometry Measurement Scenario for Localisation . . . . . . . . . . 119

4.4 a Actual odometry measurement scenario . . . . . . . . . . . . . 119

4.4b Action model particle propagation . . . . . . . . . . . 119

4.5 Laser Range Measurement Scenario for Localisation . . . . . . . . . . 120

4.5a Possible candidate state given high weight . . . . . . . . . 120

4.5b Possible candidate state given low weight . . . . . . . . . 120

4.6 Uniform Laser Range Sensor Model for Localisation . . . . . . . . . . 121

5.1 Blueprint of Simulated Robot . . . . . . . . . . . . . . 131

5.2 Test Environments . . . . . . . . . . . . . . . . 132

5.2a Symmetric Environment . . . . . . . . . . . . . 132

xvii 
5.2b Office-like Environment . . . . . . . . . . . . . 132

5.3 Emptiness Plausibility Maps Created With No Sensor Noise . . . . . 135

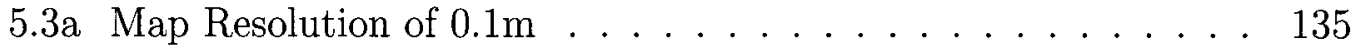

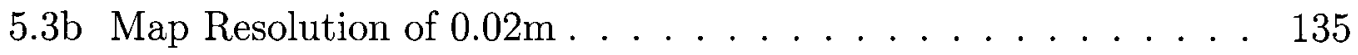

5.4 Maps Created by Test Case 1-1 . . . . . . . . . . . . . . . . 137

$5.4 \mathrm{a}$ Occupancy Support . . . . . . . . . . . . . 137

5.4b Emptiness Support . . . . . . . . . . . . . . . . 137

5.4c Occupancy Plausibility . . . . . . . . . . . . . 137

5.4d Emptiness Plausibility . . . . . . . . . . . . 137

5.5 Maps Created by Test Case 1-2 . . . . . . . . . . . . . . . 138

$5.5 \mathrm{a}$ Occupancy Support . . . . . . . . . . . . . 138

5.5b Emptiness Support . . . . . . . . . . . . . . 138

5.5c Occupancy Plausibility . . . . . . . . . . . . . . 138

5.5d Emptiness Plausibility . . . . . . . . . . . . . . 138

5.6 Maps Created by Test Case 1-3 . . . . . . . . . . . . . . . 138

$5.6 \mathrm{a}$ Occupancy Support . . . . . . . . . . . . 138

5.6b Emptiness Support . . . . . . . . . . . . . 138

5.6c Occupancy Plausibility .................... 138

5.6d Emptiness Plausibility . . . . . . . . . . . . . 138

5.7 Maps Created by Test Case $2-1 \ldots \ldots$. . . . . . . . . . . 138

5.7a Occupancy Support . . . . . . . . . . . . . 138

5.7b Emptiness Support . . . . . . . . . . . . . 138

5.7c Occupancy Plausibility . . . . . . . . . . . . . 138

5.7d Emptiness Plausibility . . . . . . . . . . . . . 138

5.8 Maps Created by Test Case 2-2 f . . . . . . . . . . . . . . . . . . 139 
5.8 a Occupancy Support . . . . . . . . . . . . . . . . . 139

$5.8 \mathrm{~b}$ Emptiness Support . . . . . . . . . . . . . . . . 139

$5.8 \mathrm{c}$ Occupancy Plausibility . . . . . . . . . . . . . . . . 139

5.8d Emptiness Plausibility . . . . . . . . . . . . . . . 139

5.9 Maps Created by Test Case $2-3 \ldots \ldots$. . . . . . . . . . . . 139

5.9 a Occupancy Support . . . . . . . . . . . . . . . . 139

5.9b Emptiness Support . . . . . . . . . . . . . . . . . . 139

5.9 c Occupancy Plausibility . . . . . . . . . . . . . . . . . 139

5.9d Emptiness Plausibility . . . . . . . . . . . . . . . 139

5.10 Maps Created by Test Case $3-1 \ldots \ldots$. . . . . . . . . . . . . . 141

5.10a Occupancy Support . . . . . . . . . . . . . . . 141

$5.10 \mathrm{~b}$ Emptiness Support . . . . . . . . . . . . . . . . . 141

5.10c Occupancy Plausibility . . . . . . . . . . . . . . . . . 141

5.10d Emptiness Plausibility . . . . . . . . . . . . . . . . . 141

5.11 Maps Created by Test Case 3-2 . . . . . . . . . . . . . . . . 141

5.11a Occupancy Support . . . . . . . . . . . . . . . 141

$5.11 \mathrm{~b}$ Emptiness Support . . . . . . . . . . . . . . . . . 141

5.11c Occupancy Plausibility . . . . . . . . . . . . . . . . . 141

$5.11 \mathrm{~d}$ Emptiness Plausibility . . . . . . . . . . . . . . . . 141

5.12 Maps Created by Test Case 3-3 . . . . . . . . . . . . . . . 141

5.12a Occupancy Support . . . . . . . . . . . . . . . . . . 141

$5.12 \mathrm{~b}$ Emptiness Support . . . . . . . . . . . . . . . . . 141

5.12c Occupancy Plausibility . . . . . . . . . . . . . . . 141

5.12d Emptiness Plausibility . . . . . . . . . . . . . . . . . 141

5.13 Maps Created by Test Case $4-1 \ldots \ldots$. . . . . . . . . 142 
5.13a Occupancy Support . . . . . . . . . . . . . . . . . . . . 142

$5.13 \mathrm{~b}$ Emptiness Support . . . . . . . . . . . . . . . . . . . 142

5.13c Occupancy Plausibility . . . . . . . . . . . . . . . . . 142

5.13d Emptiness Plausibility . . . . . . . . . . . . . . . . . . 142

5.14 Maps Created by Test Case $4-2 \ldots \ldots$. . . . . . . . . . . . 142

5.14 a Occupancy Support . . . . . . . . . . . . . . . . . 142

5.14b Emptiness Support . . . . . . . . . . . . . . . . . . 142

5.14 c Occupancy Plausibility . . . . . . . . . . . . . . . . 142

5.14d Emptiness Plausibility . . . . . . . . . . . . . . . . 142

5.15 Maps Created by Test Case $4-3 \ldots \ldots$. . . . . . . . . . . . 142

5.15a Occupancy Support . . . . . . . . . . . . . . . . . 142

5.15b Emptiness Support . . . . . . . . . . . . . . . . . . 142

5.15c Occupancy Plausibility . . . . . . . . . . . . . . . . 142

5.15d Emptiness Plausibility . . . . . . . . . . . . . . . . . 142

5.16 Maps Created by Test Case $5-1 \ldots \ldots \ldots$

5.16 a Occupancy Support . . . . . . . . . . . . . . . . 143

$5.16 \mathrm{~b}$ Emptiness Support . . . . . . . . . . . . . . . . . . 143

5.16c Occupancy Plausibility . . . . . . . . . . . . . . . . . 143

$5.16 \mathrm{~d}$ Emptiness Plausibility . . . . . . . . . . . . . . 143

5.17 Maps Created by Test Case $5-2 \ldots \ldots \ldots \ldots$

5.17a Occupancy Support . . . . . . . . . . . . . . . . . 143

5.17b Emptiness Support . . . . . . . . . . . . . . . . . . . 143

5.17 c Occupancy Plausibility . . . . . . . . . . . . . . . 143

5.17d Emptiness Plausibility . . . . . . . . . . . . . . . . 143

5.18 Maps Created by Test Case $5-3 \ldots \ldots \ldots \ldots$ 
5.18a Occupancy Support . . . . . . . . . . . . . . . . 143

5.18b Emptiness Support . . . . . . . . . . . . . . . . . . . . . 143

5.18c Occupancy Plausibility . . . . . . . . . . . . . . . . 143

5.18d Emptiness Plausibility . . . . . . . . . . . . . . . . 143

5.19 Maps Created by Test Case $6-1 \ldots \ldots$. . . . . . . . . . . 146

5.19 a Occupancy Support . . . . . . . . . . . . . . . . 146

5.19b Emptiness Support . . . . . . . . . . . . . . . . . . . . 146

5.19 c Occupancy Plausibility . . . . . . . . . . . . . . 146

5.19d Emptiness Plausibility . . . . . . . . . . . . . . 146

5.20 Maps Created by Test Case 6-2 . . . . . . . . . . . . . . . . 146

$5.20 \mathrm{a}$ Occupancy Support . . . . . . . . . . . . . . . 146

5.20b Emptiness Support . . . . . . . . . . . . . . . . . . . . . . 146

5.20c Occupancy Plausibility . . . . . . . . . . . . . . 146

5.20d Emptiness Plausibility . . . . . . . . . . . . . . . . 146

5.21 Maps Created by Test Case 6-3 . . . . . . . . . . . . . . 146

5.21a Occupancy Support . . . . . . . . . . . . . . . 146

5.21b Emptiness Support . . . . . . . . . . . . . . . . . 146

5.21c Occupancy Plausibility . . . . . . . . . . . . . 146

5.21d Emptiness Plausibility . . . . . . . . . . . . . . . . 146

5.22 Occupancy Support Map Created Following Office Mapping Test Case 1147

5.23 Emptiness Support Map Created Following Office Mapping Test Case 1148

5.24 Occupancy Plausibility Map Created Following Office Mapping Test Case $1 \ldots \ldots \ldots \ldots \ldots \ldots$

5.25 Emptiness Plausibility Map Created Following Office Mapping Test Case $1 \ldots \ldots \ldots \ldots \ldots$. . . . . . . . . . . . . . . . . . . 
5.26 Illustration of Similar Locations and Distinguished Similar Locations 156 5.26 a Similar Locations . . . . . . . . . . . . . . . 156

$5.26 \mathrm{~b}$ Distinguished Similar Locations . . . . . . . . . . . . . 156

5.27 Example of MCL Settling on One Possible Location from Test Case

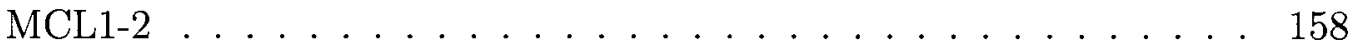

$5.27 \mathrm{a}$ Step $1 \ldots \ldots \ldots \ldots$

$5.27 \mathrm{~b}$ Step $4 \ldots \ldots \ldots \ldots \ldots$

5.28 Example of CPF-MCL Keeping Symmetric Locations from Test Case $\mathrm{CPF}-1 \ldots \ldots \ldots \ldots \ldots \ldots$

$5.28 \mathrm{a}$ Step $5 \ldots \ldots \ldots \ldots \ldots$

5.28 bStep $10 \ldots \ldots \ldots \ldots$

5.29 Example of U-MCL Failing in a General Environment . . . . . . . . . 159

5.30 Chart Summarising Single Run Success . . . . . . . . . . . . . 167

5.31 Summary Charts for Repeated Runs Test Cases . . . . . . . . . . . 170

5.31a Effect of Sample Size on Success of Repeated Runs . . . . . . . 170

5.31b Effect of Sample Size on Relocalisation . . . . . . . . . . . . . 170

A-1 Detailed Software Architecture . . . . . . . . . . . . . . 205

A-2 Simulator Screenshot . . . . . . . . . . . . . . 206 


\section{Chapter 1}

\section{Introduction}

The idea of mechanized entities to perform all types of work has become popular in recent times. The word "robot" was coined in 1923 to describe such entities, while serious research in topics of this field has been noted as far back as the 60's [79]. Early robots were arm manipulators restricted to a fixed location, typically doing repetitive mechanical assembly. Present time robots, however, are typically complex autonomous mobile robots that interact and learn within their environment. Commercially developed robots include Honda's ASIMO humanoid robot and Sony's Aibo robot dog. Robots developed by the academic community are typically showcased in various conferences and competitions, such as the Robocup competition, whose eventual goal is to "...develop a team of fully autonomous humanoid robots that can win against the human world champion team in soccer..." [105].

The completion of that goal may appear to be a frivilous affair; however, the challenges involved will result in the advancement of many fields, such as development of autonomous agents, multi-agent collaboration, real-time reasoning, robotics and sensor fusion in a dynamic environment. Indeed, various aspects of an autonomous 
mobile robot, such as the mechanical, the electrical, and the intelligence, require involvement from all disciplines of engineering, computer science, and even cognitive sciences. Advances in these fields can lead to robots that have a wider impact on important social issues such as automated mine detection and removal systems for mine fields around the world.

A typical autonomous mobile robot is equipped with sensors, actuators, and some processing capability. Sensors such as sonar or laser collect pertinent observations about the environment for the processing unit of the robot. Actuators such as motors, and arm joints then act upon the environment on the command of the processing unit. The processing unit has to independently reason, plan, and execute actions to complete its tasks based on the collected sensor data, and the capabilities of its actuators. As the robot is mobile, most of its designed task goals involve movement in its environment. Mail delivery is a possible goal of a mobile robot, where the robot must start from one area, and move intelligently in the environment to deliver mail to another area.

Hence, robot localisation is a very important part of the reasoning process for most mobile robots, referred to as "the most fundamental problem to providing a mobile robot with autonomous capabilities" by Cox [30]. Intuitively, a robot whose location is not fixed cannot plan a path to its destination if it does not first know its location within the environment. The idea of localisation leads to the idea of mapping, another fundamental problem in mobile robotics. It is not possible for a robot to localise itself if it does not initially have a map of the environment. For a truly autonomous mobile robot, it must be deployable in a completely new and unknown environment. The robot must therefore create a map of its environment, and localise itself within that map. In such a situation, both problems are exacerbated, creating a "chicken and 
egg" situation. A robot cannot map without knowing where it is, but a robot also cannot localise without first having a map. There have been many approaches to these problems, and they are linked to the robot architecture. The different ways in which different robot architectures deal with these problems are introduced in the next section.

\subsection{Robot Architecture}

The traditional robot architecture is similar to the Von-neumann architecture using the sense-model-plan-act paradigm, shown in Figure 1.1, as adapted from [137]. The robot must first collect sensory data, interpret the observations and merge them into an internal world model. The planning module then works to infer different information, such as robot location, and plan the next action for the action module to execute. Different abilities of the robot are distinctly separated into different modules, and the modules run in a serial fashion one after another.

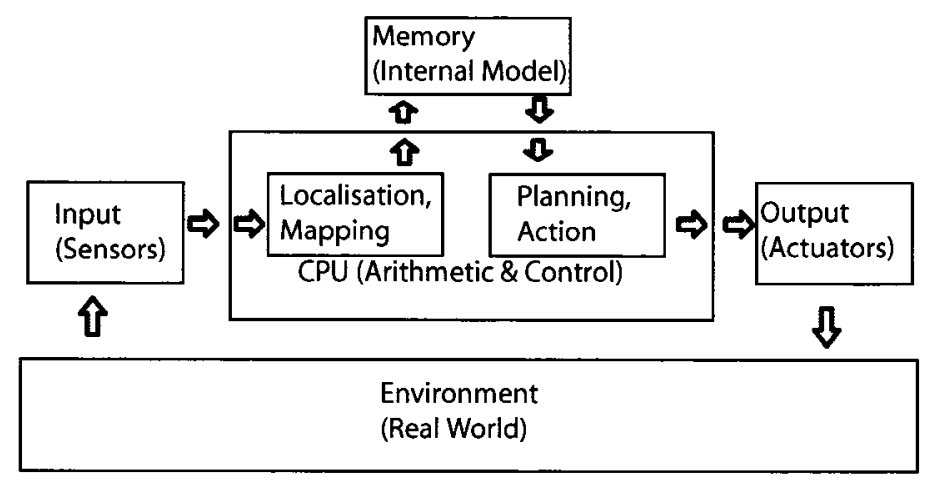

Figure 1.1: Traditional Robot Architecture

This is a top-down architecture that tries to optimise system-level goals and works using a detailed internal world model represented by logic-based formalism. Due to 
this model representation, such robots have a highly deliberative component and offer a more direct path to robots with high level cognitive abilities such as logical reasoning. A robot that is representational of this architecture is Shakey, the robot developed at the Stanford Robotics Institute in the early 80's [94].

The key problem with this approach is the assumption of a perfect world model. By using a logic-based representation, it is implicitly assumed that the model reflects the environment accurately in its entirety using logical assertions that are either true or false, with no other alternatives. Due to the logical nature of the model, it is implicitly assumed that the model of the environment is a perfect model where all necessary details are known with absolute certainty. As critiqued by Brooks, [12], this architecture is viable only in a highly engineered static environment due to the need for both the modelling and planning stage to be completed before sensory observations have any effect on robot motors. This "Von Neumann bottleneck" prevents the robot from making plans in a timely manner. While the processing unit is busy creating a plan, the robot is unable to use its sensor units to detect changes to the environment due to external forces. This prevents the robot from keeping an updated model of the environment, and the plans created by the robot are possibly based on an outdated model, thus invalidating the plan. Suppose the robot plans to move straight because its internal model indicates that the path is collision free. In the time the robot plans that path, a separate robot may move in the way of the planned path thereby invalidating the originally collision free path. This problem of having an inconsistent model prevents consistent success for mapping and localisation in a dynamic environment. Other problems include the complexities of inferring mapping and localisation information from a logic-based model, as well as the problem of using sparse, noisy sensor data to create a "perfect model" of the environment. 
With the perceived failure of the traditional architecture, various forms of reactive, highly parallel architectures were proposed. Reactive architectures promote the decomposition of the robot's overall system behaviour into smaller sub-behaviours. These sub-behaviours are typically independent of, or loosely coupled with each other, and are easily distributed among multiple processors. Interaction between execution of these prioritized sub-behaviours work together to produce complex emergent behaviour that is equivalent to the system level goal. Since sub-behaviours are optimised without regard to the overall system behaviour, this is therefore a bottom-up architecture.

The subsumption architecture proposed by Brooks [13] was the most well known of such architectures. "He who deliberates fully before taking a step will spend his entire life on one foot" is an old Chinese proverb that is representative of the architecture. The subsumption architecture addresses various problems with the classic approach. With the assertion that "the world is its own best model," [11] no model of the world is kept by the robot. Robots designed using this approach attempt to sense all the information they need directly from the environment, rather than keep an inaccurate model of the world.

Though there exists no central integrated model of the real world, there has been success in creating and localisation using a distributed map representation of the environment [81], a feat previously considered by many to be impossible for a reactive architecture with no central model. Though such complex emergent behaviour is demonstrably possible with a reactive architecture, it also shows that successful reactive robots require careful behavioural decomposition with many domain specific assumptions. They are designed for a specific type of environment, and are not easily adaptable. 
While the failing of the traditional architecture is its assumption of a perfect world model with a slow deliberative planning process, the failing of the reactive architecture is its assumption of perfect perception. In many cases, the environment is not fully accessible to the types of sensors a reactive robot may have, and the issue of inaccurate sensors and actuators still exists.

Contemporary architectures typically mix features from both architectures. Simple behaviours that need to respond in a timely manner are developed using a reactive architecture, such as collision avoidance. Complex behaviours are developed and planned using the traditional sense-model-plan-act paradigm using a centralised model. A major departure from the traditional architecture, however, is movement away from the use of logic-based model representations for the model.

This may reflect habits from a recent time when analytic approaches were more feasible and seemed more elegant because computer memories were too small to easily handle numerical arrays of a few thousand to a million cells. I think the reluctance is no longer appropriate. - Moravec, [89]

Contemporary robots typically make use of probabilistic world models that explicitly provide mechanisms to deal with uncertainty. This accounts for the impossibility of perfect models, and perfect perception in real world dynamic environments.

In many applications of robotics, such as industrial automation, and autonomous mobility, there is a need to represent and reason about spatial uncertainty. In the past, this need has been circumvented by special purpose methods such as precision engineering, very accurate sensors and the use of fixtures and calibration points. While these methods sometimes 
supply sufficient accuracy to avoid the need to represent uncertainty explicitly, they are usually costly. An alternative approach is to use multiple, overlapping, lower resolution sensors and to combine the spatial information (including the uncertainty) from all sources to obtain the best spatial estimate. This integrated information can often supply sufficient accuracy to avoid the need for the hard engineered approach - Smith, Self, and Cheeseman (1990) [120]

\subsection{Contemporary Robot Mapping and Localisa- tion}

Mapping is the act of creating an internal model of the environment with data pertinent to environment layout and structure; this specific type of model is known as a map. Two general classes of maps in popular use are topological based maps, and grid based maps. Most maps incorporate different manners to handle uncertainty to create a map that is tolerant of sensor errors, such as Bayesian reasoning, evidential reasoning, and fuzzy methods.

Though maps can account for sensor error, it is difficult to account for motion error directly in the model. Motion error accumulates over time, and invalidates the model. To minimize motion error, the robot needs to localise itself within the map.

Localisation allows a mobile robot to situate itself in its environment, given that it has a map of its surroundings a priori. The robot may know its starting position, which makes localisation a tracking issue. If the robot did not know its starting position, it is considered global localisation whereby the robot has to localise itself somewhere in its map before being able to track its movement through it. 
Certainly, with technological advances such as the Global Positioning System (GPS) that makes use of external means to determine location anywhere on earth, one might wonder if other techniques for localisation are necessary. In fact, a military grade GPS sensor can localise itself anywhere in the world with sub-meter accuracy [77]. GPS, however, only works well with a clear line-of-sight with satellites providing localisation information; thus, GPS does not function as accurately where this direct connectivity does not exist.

As mentioned before, localisation is an integral part of a mobile robot's planning process as it strives to complete its tasks. While there are countless methods published on robot localisation, most convenient methods that do not require any modification to the environment which have found success are variants of Kalman filtering, Markov localisation, and Monte Carlo Localisation (MCL). In fact, not only does MCL present good localisation characteristics, able to solve the problem of global localisation in particular, but it is also computationally tractable and easy to implement. Upon deeper analysis, it can be shown that all three methods have foundations stemming from Bayesian theory.

Generally, the mapping and localisation techniques that are successful have techniques to model uncertainty in the environment, the sensors, and the actuators. Using a contemporary architecture with a good blend of advantages from both traditional and reactive robot architectures, current robots are increasingly successful in dynamic unknown environments. There is not a complete lack of an internal environment model, yet the model is not completely trusted. This enables the robot to remember information that remains useful, yet realise which parts may not be reliable, and plan around it. 


\subsection{Thesis Statement}

It is clear that mapping and localisation are very important for a mobile robot. There are many solutions for these two problems whose performance may vary according to the complexity of the robot's environment. For example, the standard Kalman filter based solution to localisation can track the position of a robot in the environment well, given that the filter is seeded with the initial position of the robot, and that the environment does not change. If those two conditions do not hold, then the Kalman filter based solution will not successfully localise the robot.

This thesis investigates the performance of popular mapping and localisation techniques by evaluating simulation results. The mapping and localisation problems are considered separately. With mapping, it is assumed that the localisation problem is solved and mapping occurs as the simulated robot undergoes ideal motion. With localisation, it is assumed that the mapping problem is solved, and a good map of the environment is known a priori.

For mapping, the evidential method to mapping described in [97] is implemented and analysed in test environments shown in Figure 1.2. The following are results of interest:

- The theoretical comparison between the evidential mapping method and the Bayesian mapping method;

- The reformulation of the evidential method in [97] to work with laser range sensors, as opposed to sonars;

- The performance analysis of the reformulated evidential method under different parameter settings for the laser sensor model, such as noise level in sensor readings. 
For localisation, the standard Monte Carlo Localisation (MCL) [34] technique is implemented, as well as the following variants: Clustered Particle Filtering MCL (CPF-MCL) [86], and the Uniform MCL (U-MCL) [130]. Initial impressions of the U-MCL method are that it does not work in general environments in which symmetry and similarities exist in different parts of the environment. Similarity in the environment causes multimodal distributions of robot location that the U-MCL cannot deal with. This is due to the re-sampling process specified for U-MCL.

CPF-MCL groups spatially similar particles into clusters of particles, and evolves each cluster using MCL. Since each cluster represents the robot's belief that it is in a particular location in the environment, this suggests that each cluster may be updated using U-MCL without complications since the method now only deals with a single location in the environment.

In this thesis, a new variant is proposed, the Uniform Clustered Particle Filtering MCL (UCPF-MCL). The UCPF-MCL uses ideas from the CPF-MCL so that the U-MCL may be applied in a general environment.

The aforementioned techniques are simulated and compared in a small symmetric map (Figure 1.2a), as well as in a larger environment modeling that of a more realistic office-like map (Figure 1.2b). The following are results of interest:

- The effect of using an increased number of particles;

- The effect of environment size, symmetry and similarity;

- The effect of sensor and motion noise;

As well, a qualitative comparison of technique complexity is also given. 


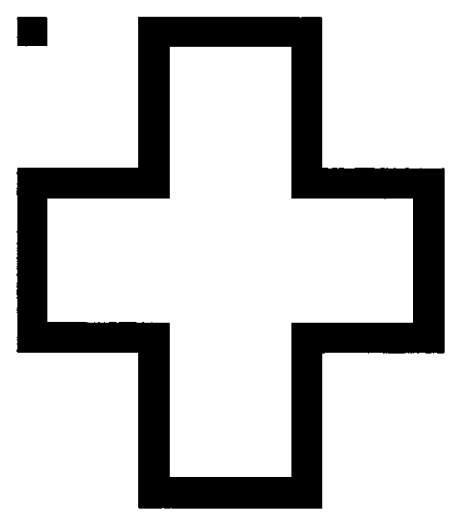

1.2a) Symmetric Environment

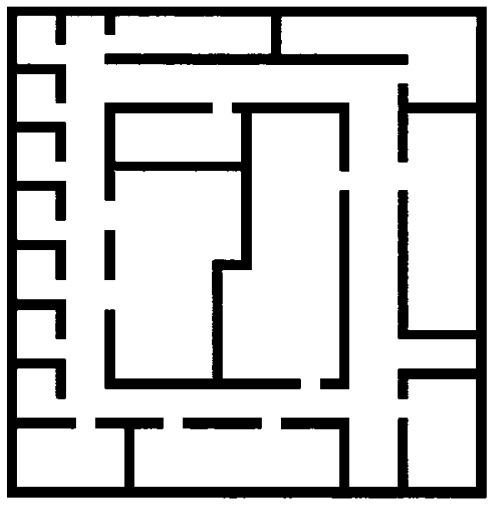

1.2b) Office Environment

Figure 1.2: Environments Used in Mapping and Localisation Experiments

\subsection{Thesis Overview}

This chapter introduced the applicability, and the challenges, of mobile robotics. The two fundamental problems in mobile robotics were briefly described, and the basics given. A brief history of mapping and localisation solutions was given, and popular contemporary solutions to these two problems were briefly mentioned.

The remainder of this thesis is organised as follows:

Chapter 2 surveys existing solutions to both the mapping and localisation problems.

General overview and discussion is given for different types of maps created by mobile robots such as the grid based, topological based, and combined maps. General overview and discussion is also given for different methods for localisation, such as Kalman filter based, Markov Localisation, and Monte Carlo Localisation.

Chapter 3 provides theoretical developments to the mapping and localisation techniques of interest. For mapping, the rationale for choosing a grid based map is given, followed by a brief review of Bayesian and Dempster-Shafer theory. The 
use of these two methods in grid based mapping is developed, and contrasted with an example.

The theory behind the localisation techniques of interest, namely MCL, CPFMCL, U-MCL, and UCPF-MCL, is then given. The development of these techniques is presented, starting with their roots in Monte Carlo sampling, importance sampling, sequential importance sampling, and sampling importance resampling. Further theoretical details are then given on MCL, CPF-MCL, U-MCL, and the new localisation technique contributed by this thesis called UCPF-MCL is finally proposed.

Chapter 4 gives details on the architecture and implementation of the simulator used to simulate the test results.

Chapter 5 develops mapping and localisation experiments that are run in the simulator to generate simulation results. The results are subsequently presented and discussed. The evidential mapping method is shown to produce good maps. The localisation methods are shown to produce good localisation results, but the UCPF-MCL method performs noticeably better.

Chapter 6 provides general discussions, ideas for future research, and concludes the thesis work.

Some detailed results are available in the attached appendices. 


\section{Chapter 2}

\section{Background}

A fully autonomous mobile robot is one that should be able to function, without human control, for a lengthy period of time in a completely unknown environment. Though there are a multitude of issues involved in the creation of such a robot, mapping and localisation are considered by many to be the fundamental issues.

To perform any useful task that exploits the mobile capabilities of an autonomous mobile robot, the robot must be aware of various pertinent details about the environment in which it is deployed. Such awareness about the environment is encoded in a map, the medium between low level physical sensors, and higher level processes such as planning and localisation.

At a higher level, localisation is also an essential component to an autonomous mobile robot. Since the location of a mobile robot is not fixed, the robot must be able to determine its location before it can plan a path to its destination. Given a map of the surrounding environment, the robot can select probable locations of where it is situated according to sensor observations. Given enough observations, it should be possible for the robot to locate itself within the map. 
With this description, one might interpret the overall system to be as depicted in Figure 2.1a, which is inaccurate. Practical actuators do not move the robot exactly as commanded, and introduce noise into its motion. Dead reckoning cannot reliably track the robot's location and cumulative error will grow without bounds. An inaccurate map generated with such a naïve approach is shown in Figure 2.2a. Figure 2.2a shows that the map initially created with small amounts of dead reckoning error is relatively accurate, but the cumulative nature of the error results in the map shown in Figure 2.2c that barely resembles the actual environment. Figure 2.1b illustrates a possible configuration for a pure mapping robot. With ideal actuators, dead reckoning can localise the robot at all times, and an accurate map can be built with practical sensors. An alternative is to have ideal sensors, and practical actuators. Localisation can be accomplished using ideal sensor observations, leading to the construction of an accurate map. Clearly, localisation is an important component of the mapping process.

Pure localisation, on the other hand, assumes that a relatively accurate map is already available to the robot, which may be true in known environments. The structure for such a case is illustrated in Figure 2.1c.

Clearly, both the pure mapping and localising robot are not the practical situations for a realistic mobile robot that may be deployed in an unknown environment. Both robot models assume certain information to be accurate, an impossible feat in the real world. Figure $2.1 \mathrm{~d}$ illustrates the structure of a robot that recognises the necessary interaction between localisation and mapping for a robot that can be deployed in a completely unknown environment. Localisation and mapping are treated as a single process to be solved, and is commonly known as simultaneous localisation and mapping (SLAM). 


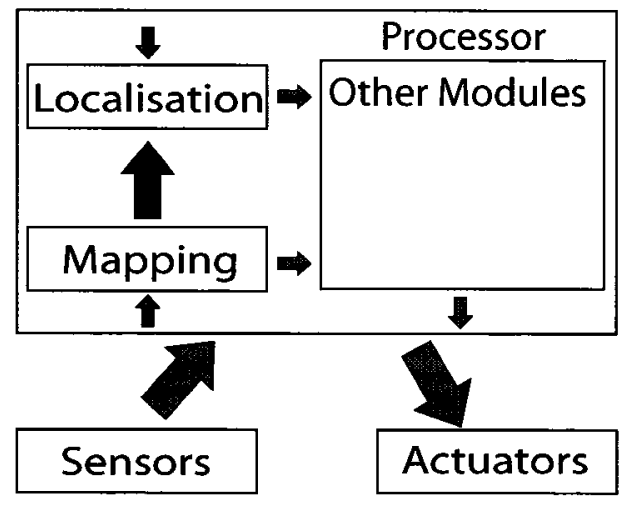

2.1a) Inaccurate Model of Mobile Robot

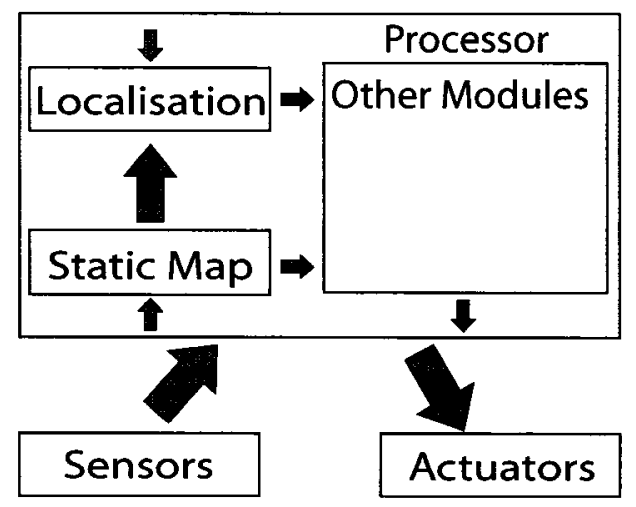

2.1c) Possible Model of a Localising Mobile Robot

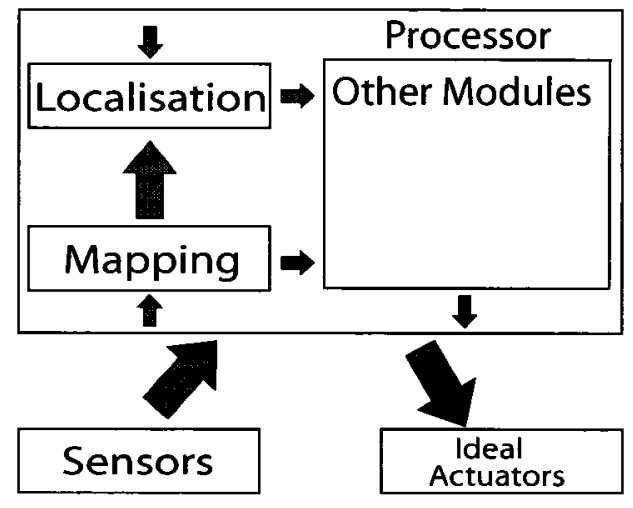

2.1b) Possible Model of a Mapping Mobile Robot

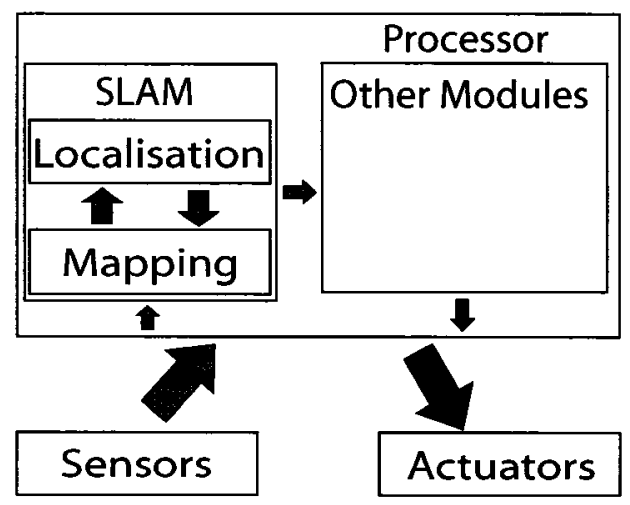

2.1d) Possible Model of a SLAM Mobile Robot

Figure 2.1: Module Interaction

\subsection{Issues in Mapping and Localisation}

Issues that complicate mapping and localisation arise from the environment itself, as well as the use of noisy sensors, and noisy actuators.

The type of environment that a robot deals with can be classified with the following criteria [108]:

Accessibility: The robot's accessibility to pertinent information about the environment to make its course of action. If the robot's sensors can sense the state of 


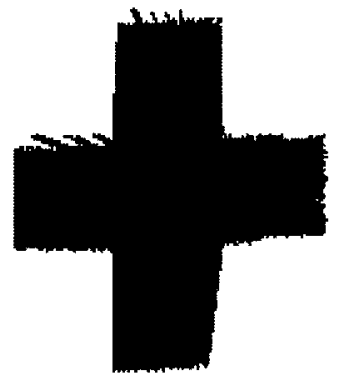

2.2a) Map Created After Small Amounts of Motion

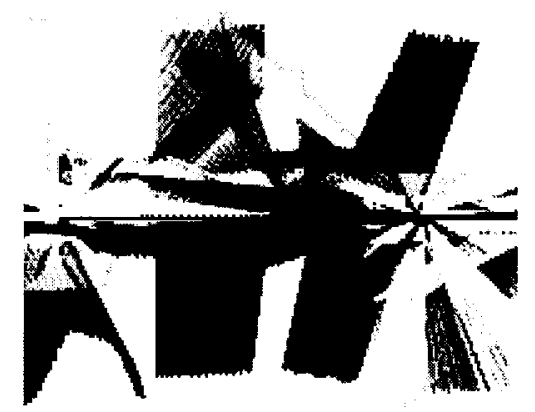

2.2c) Map Created After Full Robot Motion

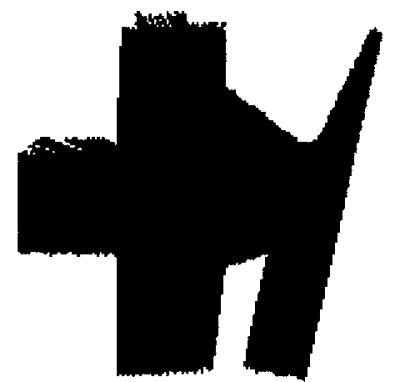

2.2b) Map Created After First Turning Motion

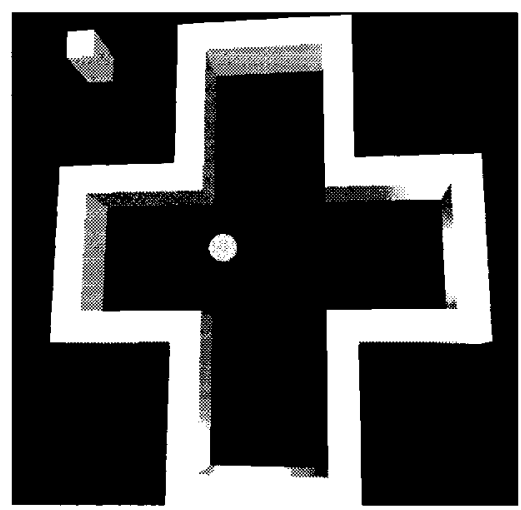

2.2d) Actual Environment After Robot Motion

Figure 2.2: Maps Created Using Dead Reckoning Without Accounting for Motion Error

the world in its entirety, then the environment is accessible. With an accessible environment, it is implied that perfect perception exists, and that no internal world model needs to be kept since required quantities can be gotten from sensor readings instead. The typical environment dealt with is inaccessible; the robot is not omniscient of required information with perfect confidence due to sensor limitations. 
Determinism: The quality of the environment that specifies whether actions, observations, and results are affected by chance. A real world environment is non-deterministic, affected by inconsistent actions, and observations whose results vary from one time instant to the next.

Episodic: Specifies whether the quality of future actions will be affected by current actions. Episodic environments are such that the task to be performed by the mobile robot can be split into smaller "episodes", or tasks, to be solved, and a poor solution to a specific episode does not adversely affect any other episode. Typical real world environments are non-episodic, in which the overall task of the mobile robot cannot be split into unrelated episodes. A poor action for a specific episode will decrease the overall quality of the solution.

Dynamicism: Specifies whether the environment changes due to forces other than from the robot itself. A typical environment is dynamic with external forces such as people wandering around the environment changing the state of various objects, such as doors.

Continuity: Specifies whether the environment is fundamentally of discrete or continuous valued. Real world environments are continous valued.

The typical real world environment an autonomous mobile robot deals with is inaccessible, non-deterministic, non-episodic, dynamic, and continuous. The robot's reliance on imperfect sensors and actuators introduces many factors that contribute to the various characteristics of the environment such as:

Sensor capability: Sensors may provide sparse or dense observations about the environment, and may provide inadequate or excessive information, respectively, 
to the robot. For example, laser range sensors return the distance to a single point in space, whereas cameras return the color, brightness, and saturation of light visible through the viewable area of the camera. Also, sensor capability will be limited in range, or may be occluded.

Sensor and actuator noise: Sensor observations are normally contaminated by noise due to imperfect sensors. Actuators are also unable to execute with absolute certainty. For example, drift and slippage of motors and wheels of the robot will introduce differences between commanded robot motion and actual robot motion, contributing to dead reckoning error. These errors contribute to cumulative error of the environmental model created by the robot if not accounted for.

Data interpretation: The observations returned by the sensors will need to be interpreted into a form that is useful for the robot. For example, a single camera image may not yield any conclusive information about the environment, but the combination of many camera images of the same location from different view points can create a 3-D model through the use of stereoscopy.

Data association: The robot needs to be able to determine whether the observations coming in from the sensors have been seen before, or whether the observations are new. Viewing this from a high level of abstraction, if the robot sees a door, it needs to determine whether it is a door it has seen before, or whether it is a new door that needs to be incorporated into the map model.

Approximative nature: Mobile robots and the environments they work in possess complex dynamical properties, and can only be modeled approximately, which introduces another source of error into the model. 
Real-time requirements: Mobile robots have hard real-time requirements since they operate in a dynamic world.

These are important factors to be accounted for in the mapping and localisation process. The robot must collect observations about the environment under these constraints, and create its models of the environment accordingly. These models should contain the necessary information for the robot to make decisions based on its observations, and are the basis for being truly autonomous without external intervention.

\subsection{Mapping}

Models that contain the necessary information for navigational issues, such as environment layout and structure, are called maps. Maps for structured indoor environments are typically 2-D overhead views of the building structure, much like building blueprints. Such environments are assumed to be vertically indistinct, meaning that features, such as walls, are flat and textureless. Therefore, the distance measured to the feature is the same, regardless of the height at which the distance is measured at. Therefore, a 3-D environment model is not needed, and collapseable into a 2-D overhead map. In this section, pure mapping will be considered with little consideration towards interaction with the localisation component, even though it has been emphasised that mapping is intrinsically linked to localisation. It is expected that a pure mapping strategy can be augmented later to include a localisation component, thus, solve SLAM as a single process. Pure mapping methods need only account for environmental and sensory issues without regards to motion error.

The choice of map to create determines the types of applicable mapping methods. Most maps may be classified as either grid based, or topological based representa- 
tions [122]. Both methods have different advantages and disadvantages (Table 2.1 as extracted from [122]), and are virtually complements (direct opposites) of each other.

\begin{tabular}{|c|c|}
\hline Grid based & Topological based \\
\hline $\begin{array}{l}+ \text { easy to build, represent, and maintain } \\
+ \text { recognition of places (based on geome- } \\
\text { try) is non-ambiguous and view point } \\
\text { independent } \\
+ \text { facilitates computation of shortest } \\
\text { paths } \\
\text { - planning inefficient, space-consuming } \\
\text { (resolution does not depend on the } \\
\text { complexity of the environment) } \\
\text { - requires accurate determination of the } \\
\text { robot's position } \\
\text { - poor interface for most symbolic prob- } \\
\text { lem solvers }\end{array}$ & $\begin{array}{l}+ \text { permits efficient planning, low space } \\
\text { complexity (resolution depends on the } \\
\text { complexity of the environment) } \\
+ \text { does not require accurate determina- } \\
\text { tion of the robot's position } \\
+ \text { convenient representation for symbolic } \\
\text { planner/problem solver, natural lan- } \\
\text { guage } \\
\text { - difficult to construct and maintain in } \\
\text { large-scale environments if sensor infor- } \\
\text { mation is ambiguous } \\
\text { - recognition of places often difficult, sen- } \\
\text { sitive to the point of view } \\
\text { - may yield suboptimal paths }\end{array}$ \\
\hline
\end{tabular}

Table 2.1: Advantages and disadvantages of grid based and topological map representations

\subsubsection{Grid Based Map}

A grid based map represents the environment by splitting the environment into small cells. These cells are typically uniformly sized, and are laid out in a grid pattern. Each cell corresponds to an area in the physical environment, and maintains a numerical value that represents the "occupancy" of that area. These types of map models are therefore called occupancy grids, and papers by Elfes and Moravec [41, 89] are representative of the approach. The ideas have since been used and modified by many researchers $[10,14,15,46,56,82,97,124,134]$.

This type of model also presents a common platform for data fusion from a wide 
variety of sensors that can sense the occupancy of the environment, and map it into the model. This platform works not only with common sensors such as lasers and sonars, but also exotic sensor configurations. For example, [109] even uses a single monocular camera to perform its mapping. A grid based map is a simple direct representation of the environment, and there is a one-to-one mapping between each cell and an area of the environment. This mapping makes it easy to update the occupancy of cells when sensor observations of their mapped areas are collected. There are, however, some issues regarding this type of model such as model parameters and model updates.

\section{Map Parameters}

There are a few different properties to be chosen for a grid based map. These include cell shape, cell size, and cell layout.

Most implemented grid based maps follow Elfes and Moravec [41, 89] in choosing square cells to split the environment, in reference to a typical grid and is similar to how digital images are composed of tiny picture elements (pixels). Though the popular shape of cells for grid based maps is normally rectangular in shape, there has been some proposed grid based maps that use alternative cell shapes. In [25], for example, Chatila et al. used sets of polyhedra to divide the environment.

The cell size is another parameter of the grid based model that must be chosen with care. Once chosen, it is not changeable online in typical implementations. A large cell size will alleviate memory and processing concerns, since it will require less cells to represent a particular area when compared to smaller cell sizes. The resolution of the map, however, degrades with increases in cell size. This idea is emphasised using the example shown in Figure 2.3a that has an exaggerated cell size; the various cells are much too large for the area, and cannot be classified with definity to be 
occupied or unoccupied since the cell technically has qualities of both states. Should the cell be classified as unoccupied, the robot will encounter unforeseen collisions if it plans a course through that cell (Figure 2.3b). Should the cell be classified as occupied, the robot will regard the free space in that cell as occupied, and not be able to use the free space in its movement plan. This may lead to a situation whereby the robot will believe there is no path to the destination (Figure 2.3c), when in fact a path may exist if the model is of higher resolution (Figure 2.3d). Thus, a small cell size is advantageous in that it can represent environment occupancy at a higher resolution. This will, however, require more memory and processing power to retain and update information about the increased number of cells. As mentioned earlier, typical implementations of the grid based method make use of a single cell size that is not changeable online. There are, however, implementations such as $[24,84]$ that make use of variable sized cells as part of their model. In [24] for example, they make use of a quadtree structure to maintain a multi-resolution grid-based map of the environment. With such an implementation, memory usage is minimized such that large areas of free-space and occupied space are represented with a single large cell, whereas smaller sized cells are used for areas that require that level of accuracy.

Depending on the shape and size of the cell, there may exist different ways to lay out the cells of the model. With a typical uniformly sized square cell model, the only logical arrangement possible is a standard uniform array grid based model. With the variably sized rectangular cell quadtree implementation of the model mentioned previously, the cell layout will be different for different environment configurations. An example of this effect is depicted in Figure 2.4. 


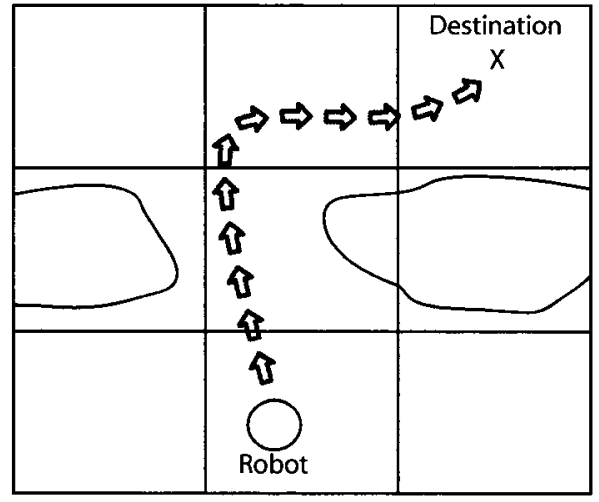

2.3a) Environment with grid cell overlay

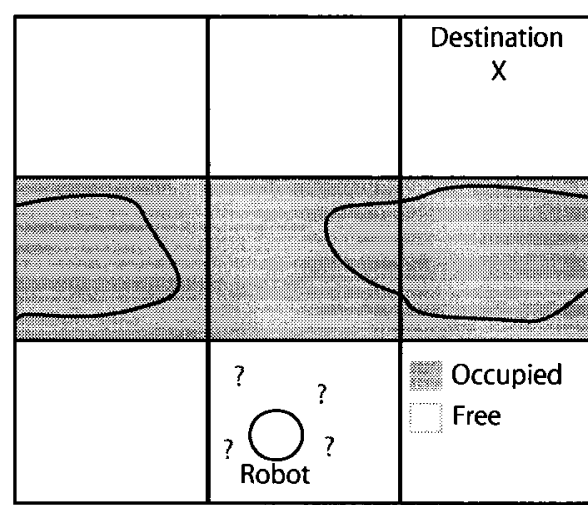

2.3c) Possible map occupancy without path to destination

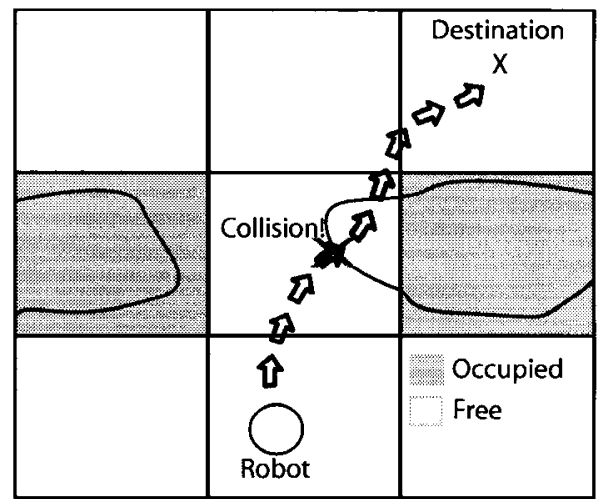

2.3b) Possible map occupancy with path to destination

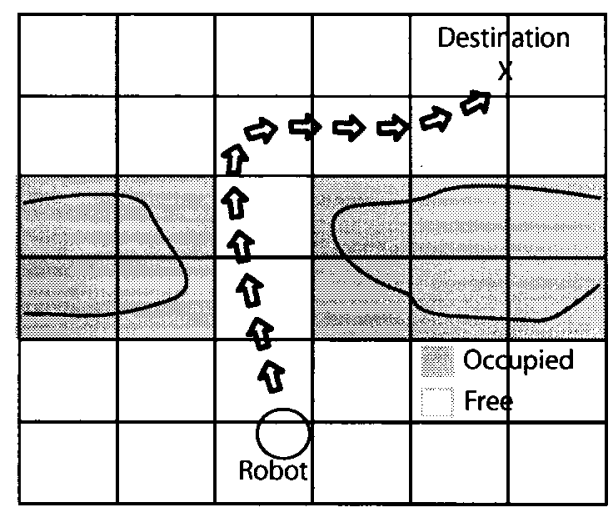

2.3d) Higher resolution map with collision free path to destination

Figure 2.3: Low resolution map with large grid cell size

\section{Map Updates}

Once the map and its general structure has been created, the map will be updated upon the arrival of sensor observations. Two major issues relating to map updates are the issues of correspondence and data fusion.

Determination of the mapping between the model cells and the area of the environment they represent is a correspondence issue. Though there is ideally a one-to-one mapping between the model cells and the environment, it is impossible to find the 


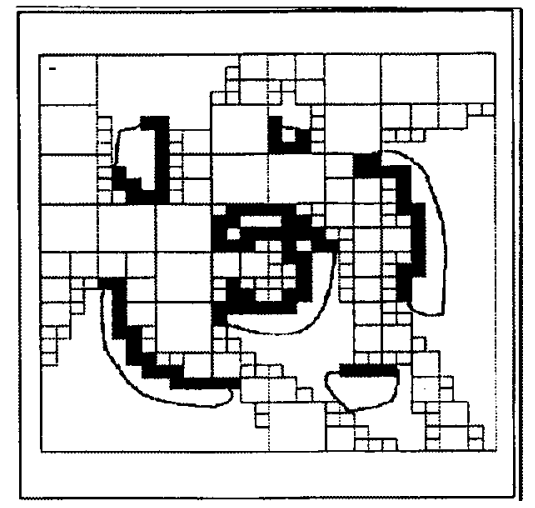

Figure 2.4: Multiresolution map using quadtree representation from [24]

exact mapping given inaccurate observations and motion, and can only be estimated. Uncertainty in observations and motion will be considered separately, although they will both result in the same problem. Essentially, it is impossible to find the exact cell that corresponds to a sensor observation.

With uncertainty in the sensor observations, assuming absolute certainty in the robot motion, the robot will only be able to select a group of cells that may be described by the observations. A simplified example is shown in Figure 2.5a whereby the robot occupies a single cell, and there is an occupied cell three cells away. Suppose the robot has a sensor that can detect the range to an object with an uncertainty of one grid cell, then Figure 2.5b shows a possible grid based model the robot might build. The robot cannot know whether the sensor information is overestimated, underestimated, or is perfectly accurate.

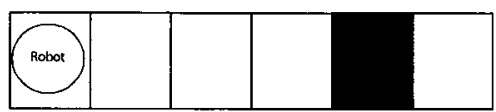

2.5a) Simple environment with grid overlay

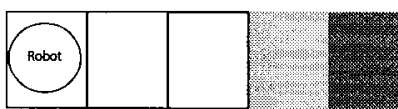

2.5b) Possible map created with noisy range sensors

Figure 2.5: Examples of maps created with noisy sensors and ideal motion 
With uncertainty in the robot motion, assuming ideal sensor observations, the robot will again be unable to select the proper cells corresponding to the ideal observations. This can be shown using the same situation as with the previous example (Figure 2.5a) whereby the robot occupies a single cell and starts with absolute certainty in the illustrated cell. If rightwards motion had an uncertainty of one grid cell, then Figure 2.6a, shows possible actual states of the robot after a commanded one grid cell movement to the right. Figure $2.6 \mathrm{~b}$ shows corresponding maps that might be created.

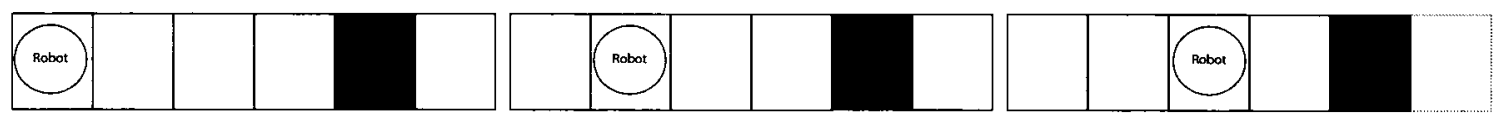

2.6a) Possible states after uncertain rightwards motion

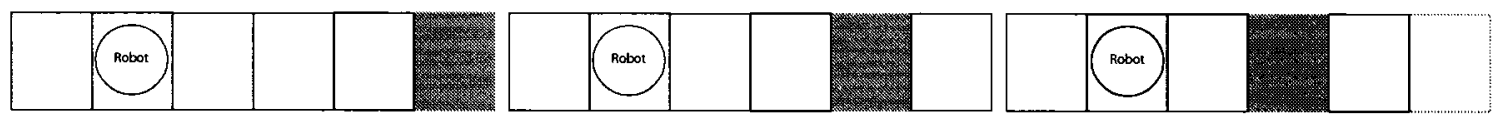

2.6b) Corresponding maps created after motion

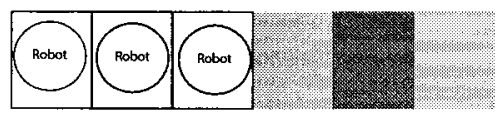

2.6c) Possible map state after combination

Figure 2.6: Examples of maps created with ideal sensors and noisy motion

Combining the different possible maps, as shown in Figure 2.6c, both the occupied space and robot location is unknown. This is the beginnings of the SLAM problem formulation, since both motion and location are unknown, and the mapping problem is exacerbated. Due to the assumption of ideal sensors, a perfect map can be reconstructed through calibration with the ideal observations after the noisy motion, so long as a perfect map of the environment prior to the noisy motion is available.

As has been shown, uncertainty in both sensor observations and robot motion both result in an uncertain mapping correspondence, and a number of candidate cells 
which may correspond to the sensor observations collected. The latter scenario with the assumption of ideal sensors is rarely considered, since such sensors do not exist, and no practical experiment can be created. The former scenario that assumes ideal motion is possible, since robots, or simply sensors, can be manually localised through manual surveying techniques so that a map can be built. Due to the ambiguity in correspondence caused by noisy sensors, cell occupancies are typically modeled using probability values, and require a method of merging sensor observations for best results.

The method used to merge sensor observations into the map has to implicitly handle issues such as multiple observations and conflicting observations to create an accurate map. The end result of the merging method is a measure of confidence in the occupancy of the cell under examination. There are many methods to merge sensor observations into a single grid based map to deal with the uncertainty described, and include ad-hoc [9], fuzzy [45], Bayesian [89], and evidential [97] methods. These methods all present different ways to deal with uncertain, contradictory, and spurious observations. The Bayesian method of updating the cell occupancy values remains the most popular method due to its mathematical basis, good performance, and early introduction.

\subsubsection{Topological Based Map}

A topological based map represents the environment by dividing it into significant areas. In general, these areas may have a distinguishing quality about them, such as a specific landmark, or be a large expanse of free space. In a typical indoor environment, such areas might be the various rooms of the building, and the corridors connecting them. The typical topological map is a graph based model, each node in the graph 
represents an area, and arcs connect areas to one another. The arcs may encode within them the information required to move between different areas, such as a direction to move towards, or metric information. Due to the freedom in choosing the manner of dividing the environment into topological sections, there is no selection of literature that would be representative of this approach. Some early work using topological maps include work by Matarić [81], and Kuipers et al. [68]. There has also been much work using this type of map [29, 42, 67, 101, 116, 133, 140].

A topological map views the environment at a higher level of abstraction which is more consistent with how a person would view an environment. Many cognitive scientists who have studied humans' robust performance in navigating through complex environments have observed that peoples' thoughts are organised in a hierarchical fashion with different layers of detail. This suggests that a topological description of a large scale environment is the most useful and powerful component for robust navigation $[80,100,117]$. If a person was to navigate based on landmarks, they might be given directions like "drive past the gas station, turn right when you see the church, my house is number eight." If a person was to navigate based on large expanse of free space, or rooms in an indoor environment, they might be directed to the room numbered eight. In all cases, a topological approach simplifies the planning process. Dividing the environment into large significant areas also allows low memory complexity, since a single node can represent that whole area.

The strength of the topological map is also its weakness; the model has poor spatial resolution. Since each node in the model represents a large expanse of free space, the robot can determine the node it is at due to landmarks or environment configuration, but it cannot determine its position more precisely than the spatial resolution afforded by the node representation. The robot will know it is somewhere 
in the large expanse of space, but not exactly where within that space.

Like the grid based map, there are similar issues regarding this type of map. The issues that occur, and their solutions, however, are linked closely with the method selected to split up the environment into topological nodes. Two major methods to divide environments into a graph based topological model are by using landmarks, and by area configurations.

With the landmark approach to environment division, landmarks are associated with different areas of the environment, represented by nodes of the topological graph. The arcs of the topological graph specifies connectivity between different areas of the environment.

With the area configuration approach to environment division, the shape of freespace in a certain area of the environment is associated with nodes of the topological graph. The arcs again represent connectivity between different areas of the environment.

\section{Landmark Method to Environment Division}

Associating different areas to different landmarks is the simplest method to arriving at a good topological map that can be maintained and updated with ease. If a particular landmark is detected, the robot is in the node that is associated with that landmark. If that landmark has not been seen before, it must be a new area where the robot has never been before. However, unless the landmarks were planted in the environment due to third party intervention (ie: RF beacons, modulated light beacons, bar codes, spot marks, infrared diodes, corner cubes as surveyed in [128] for industrial automated guided vehicles), landmark detection and selection becomes a serious problem. The robot would require a method to detect a natural landmark that can be repeatedly 
detected, selected, and be resolved to the same landmark in the various detections. This delves into a separate research area concerning repeatability, trackability, and selection of landmarks through various means, such as the method published in [131] that selects landmarks from images of the environment based on affine trackability. When automatically extracting natural landmarks to track, it is also an issue if the landmarks in the environment are not unique. If more than one node is associated with the same kind of landmark, then there is a correspondence issue. The robot cannot determine with certainty which node it is in without extra information, such as the robot's previous state.

Often, landmarks may be visible in more than one area, may not always be consistently chosen, or may not always be visible. A situation where landmarks are visible in more than one area is shown in Figure 2.7a, with different areas distinguished by the different combinations of beacons that is in line-of-sight. Figure 2.7b shows the same situation with one of the beacons obscured by obstacles. In this situation, most of the environment can still be correctly classified into the proper topological areas even with the occluded beacon. More advanced techniques to topological mapping may involve metrics, such as distances to beacons, pattern matching or searching techniques, and filtering techniques. The problem difficulty is increased if natural landmarks are used.

The previous example to environment division that associated landmark combinations to a topological node implicitly assumes that such landmarks are artificially inserted into the environment before hand. Research in this area concentrates on the case where human intervention is not required, and artificial landmarks are used. In [81] for example, the topological nodes correspond to natural landmarks like combinations of pre-defined straight wall segments. In [68], topological nodes correspond 


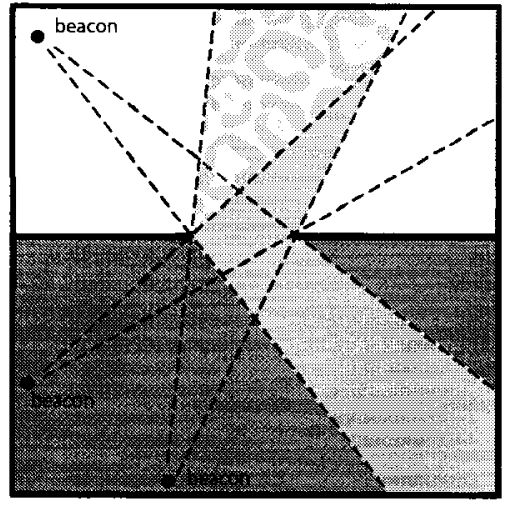

2.7a) Environment with 3 beacons, different topological areas distinguished by shading

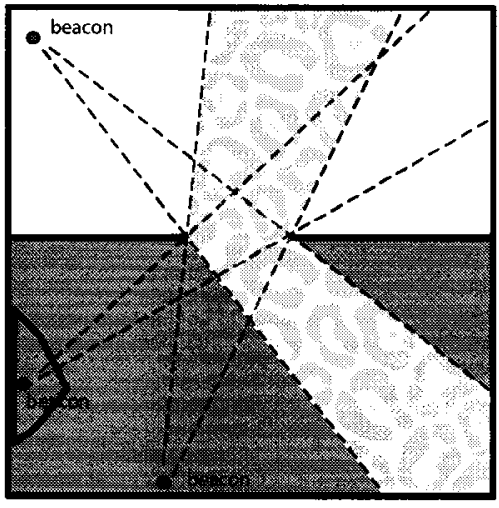

2.7b) Environment with 1 beacon occluded

Figure 2.7: Environment Division Using Landmark Beacons

to a single point that maximizes the distances to all obstacles in an area.

\section{Area Configuration Method to Environment Division}

In contrast to the landmark method, the area configuration method requires the robot to acquire a dense scan of its surrounding area to detect free-space, and associates that particular free-space configuration (ie: wall layout, shapes) with a specific node in its topological map. When the robot wishes to determine which node of the model it is in, it would acquire another dense scan of its surroundings, and attempt to match it to the different area configurations associated with every node that is in the model. The node that matches best will be the node that the robot is in. This idea is shown in Figure 2.8a. If none of the area configurations match sufficiently well, then it must be considered a new area that the robot has never been to, and will be added to the model.

A correspondence issue will arise if there are multiple areas in the environment 


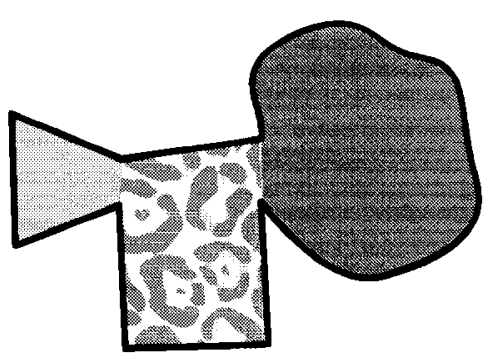

2.8a) Environment division using area shape

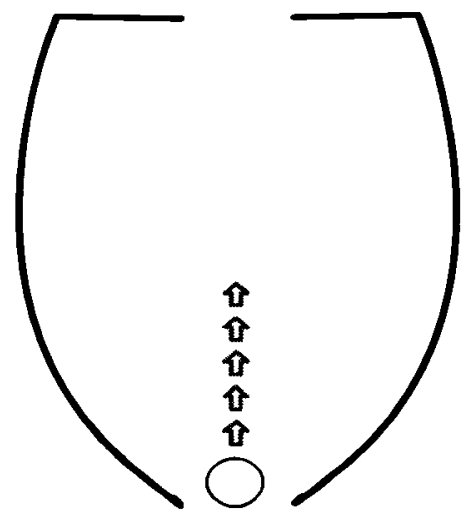

2.8c) Asymmetrical environment with robot entering from bottom

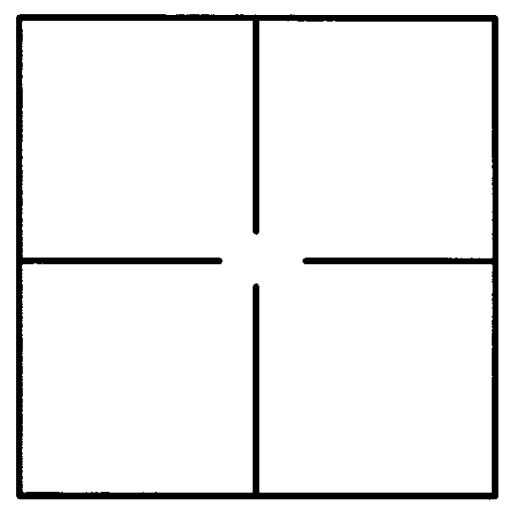

2.8b) Environment with indistinct areas
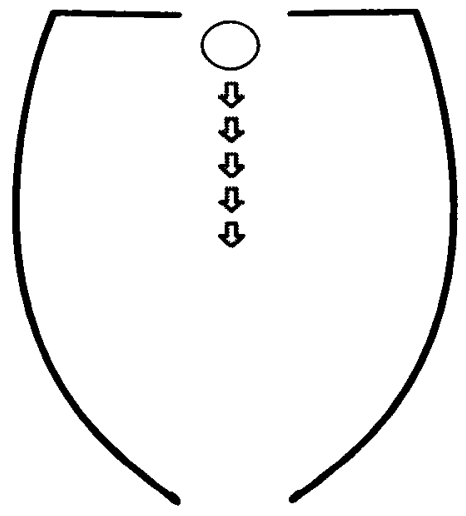

2.8d) Asymmetrical environment with robot entering from top

Figure 2.8: Environment Division Using Area Configuration

with the same approximate configuration, as in Figure $2.8 \mathrm{~b}$. The robot will be unable to determine with certainty which node it is in. Another correspondence issue is due to dense scans of the surroundings under different viewpoints. A robot entering an asymmetric room from the bottom (Figure 2.8c) will not acquire the same scan as when it enters the room from the top (Figure 2.8d). This will require a complex pattern matching algorithm to resolve the different scans under a logical rotation.

Thus, area configuration to environment division will be computationally expen- 
sive, and possibly not implementable under hard real-time constraints depending on the sensors and environment involved. Prime examples of this approach can be found in [136] and [133].

\subsubsection{Combined Maps}

Grid based and topological based maps have their respective strengths and weaknesses, but are essentially direct opposites of each other. By combining both models, it is advantageous to acquire the strengths of both models, so that the different models can complement each other. This idea has been explored by various researchers, and some results can be seen in $[2,6,122,135]$.

In [122], Thrun creates and maintains a grid based map by following the ideas of Section 2.2.1. With a grid based map, a topological map is then created from the grid based map by running algorithms to divide the environment using critical lines. Critical lines correspond to small passageways such as open doors, thus natural passageways used by people to get between logical places (ie: rooms) are extracted and used to partition the grid based map into the different areas. In combining the two maps, Thrun achieves a model that is easy to create and maintain, while deriving a topological map from the lower level grid based map that allows for ease of planning. As indicated in [122], the combined model, however, is more computationally expensive in terms of both time and space.

In [6], Bandera et al. follow essentially the same approach as Thrun [122], but they have a different method in generating the topological map online from the grid based map. The approach is hierarchical, and has a pyramid-like structure in the division of the environment. Its output is similar to the quadtree approach [27].

The combined grid based and topological map provides strengths of both types 
of maps, and offsets each individual map's weaknesses. The only weakness of the combined map is that of increased computational complexity, since more computation is necessary to derive the topological map from the grid map. Since computational power is constantly increasing, however, the advantages presented by a combined map far outweighs its weaknesses.

\subsection{Localisation}

Robot localisation is a problem in state estimation; estimating the state of the robot given measurements in the system's environment. For localisation, the state is the position and orientation of the robot in a given map of the environment, while the measurements used to estimate this state are given by the limited amount of observations provided by sensors mounted on the robot. This is an exercise in estimation due to many factors mentioned previously, the biggest of which include sparse sensor data, and noisy sensor data.

Sensors operating on mobile robots typically give sparse data about the environment. There is comparatively large volumes of the environment that are not sensed by any sensor mounted on the robot. Real sensor data are also subject to noises due to many factors such as different sensor response under different temperatures, or even the environment itself. For example, a CMOS based camera and a CCD based camera will provide different colour readings even when used in the same environment due to the different technology underlying the camera. The environment might itself contribute natural noise processes to affect the image taken by the camera, such as fog or haze.

Bayesian state estimation is the most popular method used for robot localisation. 
Being a probabilistic approach to state estimation, the Bayesian approach estimates the posterior probability density distribution over the possible states of the robot based on a prior probability density distribution, as well as the actions of the robot. This section reviews the fundamentals of the Bayesian approach.

To localise the robot, the quantities $x, y$, and $\theta$ are of interest since they describe the position and orientation of the robot in the environment. Let the state of the robot at time $t$ be $s_{t}=\left[\begin{array}{lll}x & y & \theta\end{array}\right]$. Then, the probability density distribution of the state of the robot at time $t$ is indicated by $\operatorname{Bel}\left(s_{t}\right)$. Shown in $(2.1), \operatorname{Bel}\left(s_{t}\right)$ is the conditional probability distribution refined based on the occurrence of past events $d_{0 . \ldots .}$. Therefore, the notation $\operatorname{Bel}\left(s_{t}\right)$ emphasises that the probability density distribution is the robot's belief of its location. This conditional probability distribution is fundamental to all Bayesian filters. This is a general form of the conditional distribution. Specific to robot localisation, the events of interest are the observations $o$ and the actions $a$. Following the paradigm of a sense-plan-act architecture, the observations $o$, and the actions $a$ will occur in an alternating order as in (2.2). Note that the sequence ends in an observation reading due to the fact that localisation occurs as part of the planning process in the robot architecture where $o_{t}$ is available, but $a_{t}$ has not yet occurred.

$$
\begin{aligned}
& \operatorname{Bel}\left(s_{t}\right)=p\left(s_{t} \mid d_{0 \ldots t}\right) \\
& d_{0 \ldots t}=o_{0} a_{0} o_{1} a_{1} \cdots a_{t-1} o_{t}
\end{aligned}
$$

For an autonomous mobile robot, it would be computationally beneficial to have a recursive update equation for state estimation. This update equation is derived in (2.6). Initially, the theorem of total probability (2.3) is substituted into Bayes' rule (2.4), and that is subsequently used in (2.6a). The Markov assumption (2.5), that 
the future state is independent of the past states and observations given the current state, is used in (2.6b) and (2.6c).

$$
\begin{aligned}
& p(x)=\int p(x \mid y) p(y) d y \\
& p(x \mid y)=\frac{p(y \mid x) p(x)}{p(y)}=\frac{p(y \mid x) p(x)}{\int p\left(y \mid x_{1}\right) p\left(x_{1}\right) d x_{1}} \\
& p\left(o_{t} \mid d_{0 . . t} s_{t}\right)=p\left(o_{t} \mid s_{t}\right) \\
& \operatorname{Bel}\left(s_{t}\right)=p\left(s_{t} \mid d_{0 \ldots t}\right) \\
& =p\left(s_{t} \mid o_{0} a_{0} o_{1} a_{1} \cdots a_{t-1} o_{t}\right) \\
& =\frac{p\left(o_{t} \mid o_{0} \cdots a_{t-1} s_{t}\right) p\left(s_{t} \mid o_{0} \cdots a_{t-1}\right)}{\int p\left(o_{t} \mid o_{0} \cdots a_{t-1} s_{t}^{\prime}\right) p\left(s_{t}^{\prime} \mid o_{0} \cdots a_{t-1}\right) d s_{t}^{\prime}} \\
& =\eta p\left(o_{t} \mid o_{0} \cdots a_{t-1} s_{t}\right) p\left(s_{t} \mid o_{0} \cdots a_{t-1}\right) \\
& =\eta p\left(o_{t} \mid s_{t}\right) p\left(s_{t} \mid o_{0} \cdots a_{t-1}\right) \\
& =\eta p\left(o_{t} \mid s_{t}\right) \int p\left(s_{t} \mid o_{0} \cdots a_{t-1} s_{t-1}\right) p\left(s_{t-1} \mid o_{0} \cdots o_{t-1}\right) d s_{t-1} \\
& =\eta p\left(o_{t} \mid s_{t}\right) \int p\left(s_{t} \mid a_{t-1} s_{t-1}\right) p\left(s_{t-1} \mid o_{0} \cdots o_{t-1}\right) d s_{t-1} \\
& =\eta p\left(o_{t} \mid s_{t}\right) \int p\left(s_{t} \mid a_{t-1} s_{t-1}\right) \operatorname{Bel}\left(s_{t-1}\right) d s_{t-1}
\end{aligned}
$$

where

$$
\eta^{-1}=\int p\left(o_{t} \mid o_{0} \cdots a_{t-1} s_{t}^{\prime}\right) p\left(s_{t}^{\prime} \mid o_{0} \cdots a_{t-1}\right) d s_{t}^{\prime}
$$

The recursive relationship (2.6d) is the basis behind all Bayesian state estimation filters. $p\left(o_{t} \mid s_{t}\right)$ is called the sensor model, and $p\left(s_{t} \mid a_{t-1} s_{t-1}\right)$ is called the action model. Popular Bayesian state estimation techniques are the Kalman filter, Markov localisation, and Monte Carlo localisation. 


\subsubsection{Kalman Filter}

The Kalman filter is an "optimal recursive data processing algorithm" [83] that estimates the current state of the system of interest. The Kalman filter is based on a linear system model, measurement noise, and system noise when developing its state estimates. The filter works with Gaussian distributed variables, and as such, can concisely describe state estimates by keeping track of their mean and covariance. Since the filter propagates both the mean and covariance in its process, it therefore does not lose any information during execution.

Many natural processes are Gaussian distributed. Non-Gaussian, independent processes may also be regarded to approach a Gaussian distribution due to the Central Limit Theorem. Therefore, the Kalman filter is a useful and efficient method for linear state estimation. When a process can be expressed as, or be approximated reasonably well, with a linear process, Kalman filters may be applied. The discretetime Kalman filter equations are presented in (2.8), and are used to estimate the model shown in (2.7). For a nonlinear process, Extended Kalman filters (EKF) may be used instead. Essentially, the EKF makes a locally linear approximation to the nonlinear process through the use of partial derivatives, making use of a truncated Taylor series expansion. An example is described in [5] where a nonlinear process is estimated using the EKF.

$$
\begin{array}{lr}
x_{k}=A x_{k-1}+B u_{k-1}+w_{k-1} & \text { Dynamics } \\
y_{k}=C x_{k}+v_{k} & \text { Measurements, }
\end{array}
$$


where $v_{k}, w_{k-1}$ are noise components, and

$$
\begin{aligned}
Q_{k} & =E\left[w_{k} w_{k}^{T}\right] \\
R_{k} & =E\left[v_{k} v_{k}^{T}\right],
\end{aligned}
$$

where $E$ is the expectation operator.

$$
\begin{array}{rr}
\hat{x}_{k}^{-}=A \hat{x}_{k-1}^{+}+B u_{k-1} & \text { Propagate state } \\
P_{k}^{-}=A P_{k-1} A^{T}+Q & \text { Propagate error covariance } \\
K_{k}=P_{k}^{-} C^{T}\left(C P_{k}^{-} C^{T}+R\right)^{-1} & \text { Kalman Gain } \\
\hat{x}_{k}^{+}=\hat{x}_{k}^{-}+K_{k}\left(y_{k}-C \hat{x}_{k}^{-}\right) & \text {Update estimate } \\
P_{k}=\left(I-K_{k} C\right) P_{k}^{-} & \text {Update error covariance }
\end{array}
$$

EKFs have been used for robot localisation, like in [72]. In that case, EKFs were used for determining state of the robot given a map containing landmark locations. This map is a combination of grid based and topological maps in that it maintains important landmarks that exist in the environment, like a topological map, but also maintains relative distances between the landmarks, acting like a grid based map that models distances. Associating detected landmarks with the proper landmarks that exist in the map, the robot then attempts to localise itself by using the EKF to calculate its position, and variance. The initial robot location must be known, otherwise the association between detected landmarks and mapped landmarks will not be successful. Therefore, this is a form of tracking.

Although the Kalman filter is an efficient and effective method for state estimation, its unimodal Gaussian assumptions restrict its applicability in global localisation where the robot might be unable to distinguish between locations of similar architecture. Work in $[3,62]$ has been done to address such situations using multiple hypothesis Kalman filters. The method allows for a series of Gaussian distributions 
to support multimodality. A hypothesis is created for each likely location, and is removed when it is determined to be unlikely due to new observations. Each hypothesis represents a Gaussian distribution around that location, and is predicted and corrected following [72].

Kalman filters perform weighted averaging. Kalman filters can also be viewed as a specific case of the Bayes filter with the following restrictions:

- $\operatorname{Bel}\left(s_{0}\right)$, the initial belief, is a Gaussian distribution

- $s_{t+1}$ is linear with respect to $s_{t}$

$$
s_{t+1}=A s_{t}+B a_{t}
$$

where $A, B$ are matrices.

- $o_{t}$ is linear combination of the state

$$
o_{t}=C s_{t}
$$

These constraints ensure that the belief propagates as a Gaussian distribution.

\subsubsection{Markov Localisation}

Markov localisation is named for its use of partially observable Markov decision process (POMDP) models. A Markov decision process has a finite set of states, a finite set of actions for each state, and transitional probabilities between states. When used for localisation, the states of the model represent possible locations of the robot in the environment, and the map given a priori to the robot is decomposed into a Markov decision process model. The distinction of being partially observable indicates that 
the robot cannot determine with certainty which state it is in given the available sensor observations. This is due to the inaccessibility of the environment given practical sensors. Therefore, a discrete probability distribution is maintained for all possible states of the robot at instants in time based on sensory data.

\section{Topological Markov Localisation}

A robot that pioneered the use of Markov localisation is Dervish, the winner of the 1994 office delivery event that was part of the 13th National Conference on Artificial Intelligence [95]. The robot's localisation technique was not yet named, but it introduced key concepts of Markov localisation as it is known today. Dervish maintained a set of possible states that it was in, and "progressed" the state set as new observations came in. Subsequent uses of Markov localisation in [21, 57, 119] were also successful, and formalised many concepts of the localisation technique used in Dervish.

The initial implementations of Markov localisation are topological approaches. The map of the environment is a topological decomposition into a Markov model with different states and transitions. The transitions are the actions possible in the state, and each action may cause the robot to end up in more than one end state due to uncertainty in actuator motion, and are governed by probabilities. The robot may reduce the uncertainty of being in a certain state by using sensor observations. If an observation supports a state, then its uncertainty is decreased. Conversely, the uncertainty is increased if an observation contradicts a state.

Due to the nature of a topological approach, even if the robot knows its state in the Markov model with relative certainty, it does not know its location within the state. The precision of the localisation technique depends on the granularity of the topological decomposition, which is typically quite large. In [57] where the robot 
traverses sewage pipes, a state of the robot may be in a pipe section between two sewage junctions (Figure 2.9a). When the robot traverses between state S11 and state $\mathrm{S} 10$, the robot has no way of knowing where it is in the long pipe until it determines that it has reached state S10; it knows, however, that it is somewhere in that pipe. In [119], however, approximate odometry data is used as part of their Markov model. In [119], Simmons and Koenig experimented with their robot Xavier by implementing a variant of the Markov localisation framework. The robot also localised based on a topological Markov model that was built manually and given to the robot prior to the experiment. Figure $2.9 \mathrm{~b}$ shows a Markov model for a corridor junction. At the corridor junction, a number of probabilities are defined for various state transitions given an action such as move forwards. As can be seen, a robot moving north from the south would move from state A to B with certainty. If in moving from A to $\mathrm{B}$, the robot decides instead to turn right, it has equal chances of ending up in state $\mathrm{C}$ or $\mathrm{D}$ due to the equal probability assignments of 0.5 . This probability assignment is due to the assumption of a non-zero turn radius, and the fact the robot might get into the wrong state due to noisy motion. The robot then maintains two possible states it might be in, allowing for a discrete multimodal distribution of robot state. It is up to subsequent sensor observations and actuation actions to reduce the certainty of unlikely states, and eventually return the robot state to a unimodal distribution. The equal probability values assigned may not seem intuitive, since the direction the robot is turning should at least receive a higher probability. However, the probability values are adjustable parameters.

Thus, the Markov model used in this work differed from other implementations in that it included metric information about distances between significant nodes in the map. A long corridor is decomposed into meter long sections, each section being 
its own state. Doing so, the robot can determine approximately where it is within the long corridor down to a 1 meter resolution. In their model (Figure 2.9c), A is the beginning of a long corridor, while $\mathrm{C}$ is the end of the corridor. For the robot to make the transition between $\mathrm{A}$ and $\mathrm{C}$, there are three nodes in between that the robot can move through before reaching $\mathrm{C}$. The transition between these three nodes are triggered by odometry measurements, thus the robot has a better idea of where it might be in the long corridor. To provide for the fact that dead reckoning is unreliable for navigation purposes, each of these three transitional nodes has a direct transition to the final node $\mathrm{C}$. This ensures that if the robot's odometry data is unreliable, but the robot detects that it has reached the end of the corridor, it can end up in the proper end state.

With this example, Markov localisation should also be seen as a form of a Bayes filter with the following characteristics:

- Discretized robot states $s_{t}$

- Existence of sensor model $p\left(o \mid s_{t}\right)$

- Existence of action model $p\left(s_{t} \mid s_{t-1}, a_{t-1}\right)$

\section{Grid Based Markov Localisation}

To extend upon the idea of better localisation resolution, Burgard et al. implemented grid based Markov localisation in [17].

With grid based Markov Localisation, occupancy grids [89, 90] are used directly as the Markov decision process model. Occupancy grids split the environment into regularly sized cells, and retain the probability that the cell is occupied. These grids are traditionally used for mapping and planning purposes as described in Section 2.2.1. 


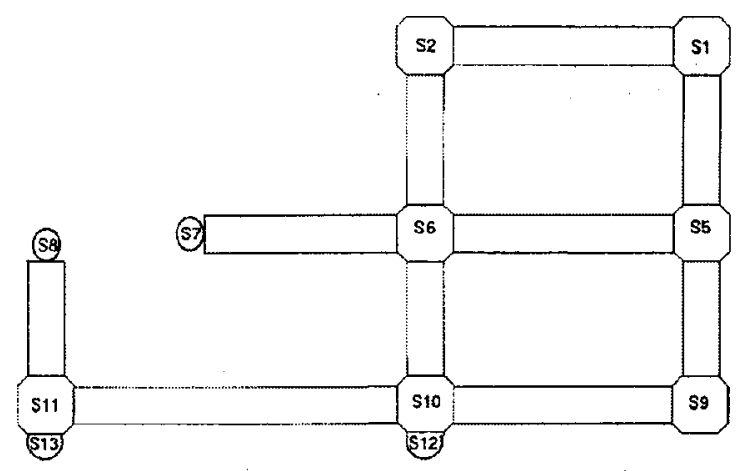

2.9a) Model of a sewage network [57]

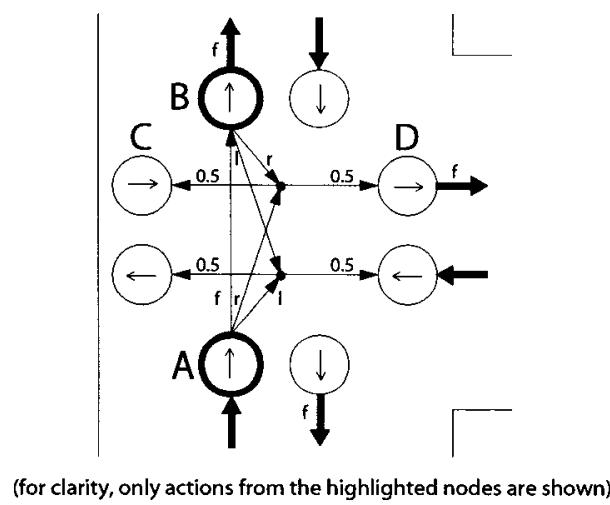

$2.9 \mathrm{~b})$ Model of a corridor junction [119]

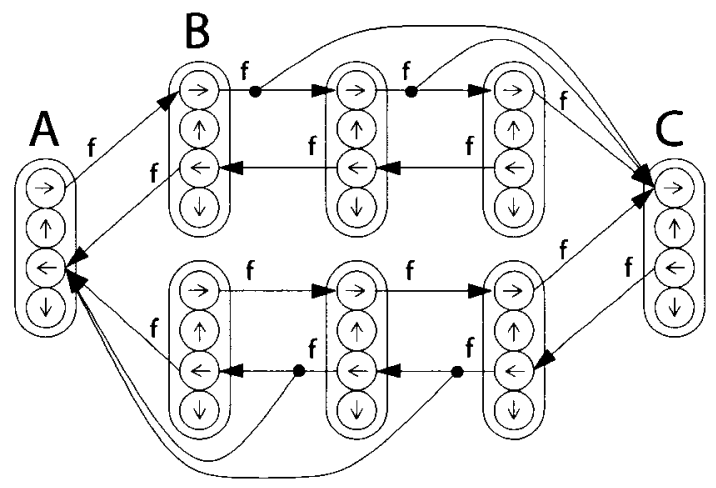

2.9c) Model of a long corridor [119]

Figure 2.9: Topological Markov Model for Markov Localisation

For localisation, however, each cell maintains the probability of the robot occupying the cell. Thus, they are called positional probability grids instead. In [17], each cell in the grid is considered a Markov state, and represents $15 \times 15 \mathrm{~cm}^{2}$. Upon reception of new sensory data, each state of the Markov model would normally need to be reprocessed to determine the likelihood of the robot being in that state. With such a decomposition of the environment, "The real world is so finely grained that any attempt to plan using a highly detailed model of reality is doomed because of enormous computational complexity" [95]. With such high resolution, the number of states grows at $O\left(n^{2}\right)$, where $n$ is the number of cells for a square map. Localisation 
becomes intractable for larger sized environments due to time and space concerns. Burgard et al. [17] makes use of two optimizations to make this problem less computationally complex, as described in [43]: a fast model for sensors, and a selective update of Markov states. The selective update provides a major computational speedup by only processing the most likely states that the robot is in. Further work was done in this area to address space concerns using an octree representation for the position probability grid [16].

\subsubsection{Monte Carlo Localisation}

Monte Carlo Localisation (MCL) was first proposed in [34] by Dellaert et al. . It is so named for its representation of probability density distributions through discrete sampling, and with this abililty, MCL directly implements the recursive update equation derived in (2.6) for state estimation. Though this was the first application towards robot localisation, the same approach has been applied to other fields and other names for the Monte Carlo approach include the bootstrap filter [50], survival-of-the-fittest [63], and the Condensation algorithm [60]. These are generally called particle filters, since each discrete sample of a probability density distribution is regarded as a particle that belongs to a probability distribution.

There exists plenty of work that makes use of the MCL process. In [52], an omnidirectional camera was successfully used to localise a robot using the MCL process even though the robot was in a very uniform, regularly structured maze-like crowded environment. Another camera vision based technique was implemented on Robocup robots in [106] using MCL. In [4], particle filters are integrated with conditional binary Bayes filters to localise the robot, and to estimate the state of doors. This modification allows the robot to estimate the state of a dynamic environment, and 
localise itself within it. Another unique application of MCL is described in [69] where the signal strength of WiFi (802.11b) wireless network cards is used as sensor observations to localise the robots. This is very useful, since $\mathrm{WiFi}$ is already used as a networking medium for communication purposes.

MCL enjoys widespread popularity due to its relative ease of implementation, accuracy, and efficiency. In fact, it is widely regarded as the de facto standard for localisation, since it is able to solve most aspects of localisation including global localisation, and tracking. Furthermore, it is also able to localise using a slightly inaccurate static map of a semi-dynamic environment due to its probabilistic approach.

The standard MCL process has been used by, and extended by, many universities and corporate laboratories. The following are some resulting variants and extensions of the standard MCL process:

- Sensor Resetting Localisation (SRL) [70]

- Mixture MCL (Mix-MCL) [127]

- Adaptive MCL (A-MCL) [54]

- Adaptive Sampling MCL (AS-MCL) [44]

- Uniform MCL (U-MCL) [130]

- Clustered Particle Filtering (CPF-MCL) [86]

There is a comparison between some of these methods in [54].

All the different particle filters make different enhancements to the general algorithm to improve efficiency or accuracy under different situations. SRL and AS-MCL are particularly interesting. SRL makes changes to the MCL process that allows a 
robot with extremely imprecise odometry to localise successfully, and was used to localise a legged Aibo robot in Robocup. The uniqueness of SRL lies in the idea of injecting highly probable particles into the particle filter according to sensor observations, an idea called sensor based resampling. Sensor based resampling, however, is not usually applicable to general environments since sensor observations cannot easily determine possible locations of the robot. AS-MCL makes modifications that allows the use of a variable number of particles to represent state distributions. A large number of particles can be used when the possible statespace of the robot is relatively large, and a small number of particles can be used when the possible statespace of the robot is relatively small. This saves computation time when a robot is well localised since there are less particles to process, and when more particles provide no advantage to the localisation process.

The variant MCL processes of interest in this thesis are U-MCL, and CPF-MCL.

U-MCL makes simplifying assumptions that the motion model and the sensor model may both be represented by uniform distributions, thereby reducing computational complexity. As shown in [130], U-MCL presents localisation results that are as good as those obtained from SRL.

CPF-MCL introduces the idea of grouping spatially similar particles together and maintaining a separate MCL process for each cluster of particles. This is due to the observation that if different clusters remain equally likely for an extended period of time, the standard MCL process tends to favour one cluster $[86,132]$. The favoured cluster tends to survive, while the other clusters are eliminated. This is a non-issue if the favoured cluster is the correct cluster, but that is not guaranteed. By maintaining a separate MCL process for each cluster, there is no premature elimination of equally likely clusters. This idea is particularly important in a symmetric environment such 
as in Figure 2.10. When a robot is at any of the locations marked with a rectangle, it should not be able to distinguish itself from any of the other marked locations due to the symmetry of the environment.

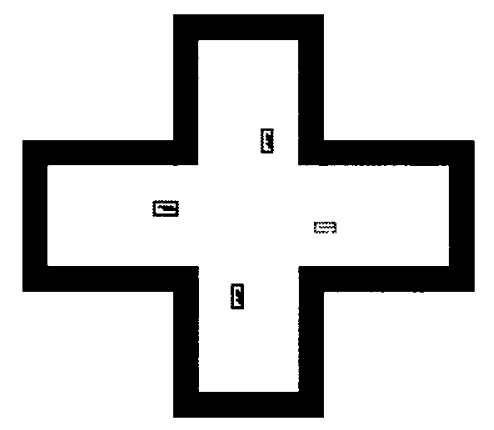

Figure 2.10: Symmetric environment illustrating importance of CPF-MCL

\subsubsection{Alternative Localisation}

Though Bayesian methods to localisation are generally the most successful methods to robot localisation available today, there exist other localisation techniques. One general category of such techniques is scan matching (model matching), while others exist that do not follow a general popularised approach.

There is an abundance of literature that document the use of scan matching techniques $[46,96,109,110]$. Essentially, the robot has a global map of the environment. While traversing through the environment, the robot creates a "local" map of its surrounding environment. Using methods related to the hill-climbing algorithm to match the local map to the global map, the robot can recover the shift and rotation required to match the maps, and use those values to correct dead reckoning error. In the literature, this model matching technique is used for "continuous localisation", 
or tracking. It is possible to modify such methods to solve global localisation, but the computational cost might be prohibitive, and will require algorithmic changes for success in a highly homogeneous environment (contains areas of similar structure). Another interesting type of model matching is explored by Iocchi et al. in the Hough domain $[51,58,59]$. Camera vision is used to extract points that lie on possible line segments, and line segments are extracted in the Hough domain. These line segments are then matched to a line segment model of the environment for localisation purposes.

An example of a localisation technique that does not follow popular methods is described by Sim [118]. The method involves camera vision as its primary sensor, and a map that is a database of decomposed visual landmarks. Landmarks with distinctive visual characteristics are chosen, and subsequently decomposed using principal components analysis $[92,99,129]$ before inclusion into the database. To localise the robot, landmarks are re-extracted from camera images, matched to the database, and a series of estimated positions are generated based on comparison between the database templates and the extracted landmarks. A Kalman filter is then used to combine the estimates to obtain a single estimate of the robot state.

\subsection{Simultaneous Localisation and Mapping}

The problems that plague mapping and localisation are combined in the simultaneous localisation and mapping (SLAM) problem. Due to data association uncertainty, environment dynamics, and computational complexity due to correlations, SLAM remains an open problem [73].

Mapping and localisation are both integral components to an autonomous mo- 
bile robot. In mapping, relatively accurate robot localisation is required to build a consistent model of the environment. In localisation, a map of the environment is needed a priori to localise the robot accurately when using noisy real world sensors and actuators. This leads to what Leonard and Durrant-Whyte call "the chicken and egg" [72] problem; which came first, the motion or the map? SLAM treats localisation and mapping as a single problem, and attempts to solve it with a single process. The formulation of the SLAM problem recognises that localisation and mapping are intrinsically linked since sensor observations must be used to infer both robot location and the model of the environment. This problem is also known as concurrent mapping and localisation (CML) [74], and was first formally introduced by Cheeseman, Smith, and Self [121].

There are distinct types of solutions to the SLAM problem, and they are Kalman filter based, expectation maximization based, and incremental based solutions.

\subsubsection{Kalman Filter Solution}

Early research and theoretical solutions to the SLAM problem began in the late 80's with seminal work performed by Smith, Self, and Cheeseman [26, 120, 121]. The mathematical formulation of the SLAM problem and initial Kalman filter based solution by Smith et al. is still in popular use today [22, 23, 36, 40, 74]. In such solutions, an Extended Kalman Filter (EKF) is used for localisation, approximating the nonlinear nature of localisation with linearization, and expressing uncertainties due to sensing and motion error with Gaussian distributions. This approach is popular due to both technical merit, and familiarity. The Kalman filter approach provides a recursive solution to problem, and is able to compute consistent estimates for both robot and landmark locations based on statistical models for robot motion, and relative 
landmark sensor observations. This approach has a sense of familiarity since it has been used extensively in many other branches of engineering.

The key to a Kalman filter based solution for SLAM is to include the state of each landmark found in the environment in the state vector. This was based on the observation that the location of every landmark in the system has to be correlated with every other landmark in the system, as well as with the location of the robot's location. Most state of the art EKF based solutions select landmarks and adjust the state vector dynamically.

In recent years, there has also been various enhancements and interesting results augmenting the original solution. In [36], Dissanayake et al. developed a proof that the Kalman filter estimated map will converge monotonically to a relative map with zero uncertainty, and that the absolute accuracy of the map and location has a lower bound dependent on the initial robot uncertainty.

Others [31, 53, 71, 73, 74, 78, 93] focused on issues related to efficient, reliable, and scaleable implementations of the solution to large scale unstructured environments. The most recent improvement to the standard Kalman based solution is the FastSLAM algorithm described by Montemerlo et al. in [87, 88]. FastSLAM decomposes the SLAM problem such that it is composed of a localisation problem, and a series of landmark estimation problems using conditional independence assumptions. In doing so, they show that it is possible to achieve a significant speed up, and improved ability to handle a magnitude more landmarks. This technique of marginalizing out some of the variables involved reduces the size of the state space, and is called RaoBlackwellisation [91]. These developments support the use of the Kalman filter based solution to the SLAM problem, in terms of mathematical rigour, and implementation efficiency. 
The weakness of this solution, is due to its formulation, and its reliance on landmarks. The need to include landmark states as part of the state vector causes the algorithm to have $O\left(n^{3}\right)$ complexity, where $n$ is the number of landmarks in the environment. Although this complexity is easily reduceable to $O\left(n^{2}\right)$, and there are modified algorithms such as the FastSLAM algorithm that reduces the complexity even more, the reliance on landmarks is itself a major issue with respects to selectability, repeatability, and occlusion of landmarks.

\subsubsection{Expectation Maximization Solution}

The Expectation Maximization (EM) solution to the SLAM problem is a relatively recent alternative to the previously discussed methods. The EM algorithm proposed in the seminal paper by Dempster et al. [35] solves maximum likelihood problems with missing data. With respect to SLAM, the model of the environment is to be determined from noisy observations and motion. In $[18,126]$, Thrun et al. reformulated the SLAM problem as a maximum likelihood problem and solved it with the Baum-Welch algorithm [7, 103].

SLAM as a maximum likelihood problem is

$$
m^{*}=\underset{m}{\operatorname{argmax}} P(m \mid d)
$$

where $m$ is a map, $d$ are collected data (ie: observations and motion controls), and $m^{*}$ is the map that is most likely as a result of the collected data. Manipulating the equation, the problem may be expressed as

$$
m^{*}=\underset{m}{\operatorname{argmax}} \int \ldots \int \prod_{t=1}^{T} P\left(o_{t} \mid m, s_{t}\right) \prod_{t=1}^{T-1} P\left(s_{t+1} \mid a_{t}, s_{t}\right) d s_{1}, \cdots, d s_{T},
$$

where $o$ are observations, $a$ are commanded actions of the robot, and $s$ are states of the robot. 
Maximizing (2.10) will give the most likely map for the collected data, however, the naïve method of maximization will require an exhaustive search in the whole set of possible maps. Fortunately, the "hill-climbing" Baum-Welch algorithm is an efficient method of finding the maximum in the likelihood space. The algorithm divides the EM algorithm into two steps: the E-step, and the M-step. In the E-step, probabilistic estimates for the robot locations are determined from the current most likely map. In the M-step, the most likely map is selected based on the refined robot location estimates. In alternating between these two steps, both the robot location and the most likely map are iteratively refined.

The EM solution thus gives the optimum solution with all given data. If new data comes in to change the optimum map, the EM algorithm is able to retract previous decisions, and make better decisions to re-maximize the likelihood. This however, carries a large computational cost, and the solution is not implementable in real time.

\subsubsection{Incremental Solution}

Incremental solutions create the environment map as sensor observations and robot motion data come in. They retain elements of mathematical computation without the rigourous Kalman filter, or statistical formalism. Most of the mapping methods described in Section 2.2 may be regarded as solutions to SLAM that do not have a localisation component, while some of them have a vague imprecise sense of location due largely to the limitation of a topological approach, such as [68, 133]. As such, uncertainty of robot location is not modeled, and a single map is created based on the current robot location, and collected data. Should the robot location be inaccurate, the inaccuracy will propagate over to the created map. It is difficult to create a 
topologically correct map in a large scale environment, especially in environments that contain "cycles". These methods are incremental maximum likelihood methods since only incoming sensor observations and robot motion are used to create the most likely map. This is a special case of the EM solution, where the expectation step is nonexistant, and the maximization occurs only for new data. Maximizations made in previous timesteps may not be retracted once they have been made, unlike with EM.

There exist incremental solutions that include a localisation component, and the ability to retract previous mapping decisions that are no longer optimum. These are hybrid approaches, since they possess properties of both incremental maximum likelihood methods, and the EM method. Such is the case in [55, 123, 125], where they create the map using the incremental maximum likelihood approach, but also maintain a posterior distribution of robot location using a particle filter. When it is detected that the incremental best guess differs sufficiently from the best guess using the full posterior distribution, the map is corrected so that the difference is minimal. The method of correcting the map "backwards in time" may be considered to be a crude approximation of the EM algorithm that is selectively performed. This approach was created as a solution to mapping cyclical environments.

Yamauchi et al. [136] make use of matching short term grid maps to the global (long term) grid maps. The rationale is that the short term grid maps describe the entire local environment of the robot, and the location of the robot within the short term grids is relatively well known since cumulative error due to noisy motion will be low. By matching this short term grid map to the global grid map, the cumulative error in the global grid map is not allowed to accumulate since the robot is constantly relocalised. Others that use a similar approach include [46]. 


\subsection{Chapter Summary}

The state of the art solutions to mapping and localisation are surveyed in this chapter.

There are generally two popular types of maps employed by contemporary mobile robots: grid based, and topological based. Each type has their own strengths and weaknesses, and are generally complementary to each other. There is research in recent times to develop combined maps that combine the strengths of the maps at the cost of computation. Such efforts derive a topological map from a grid map. A combined map is desirable, therefore a grid based map is the focus of this thesis. Chapter 3 goes into more details about grid based mapping methods. Future work can introduce a component to create topological maps from the grid map, useful for high level planning.

Many solutions to the robot localisation problem are available. The most popular ones, however, are based on Bayesian theory. Such methods perform well under uncertain sensor readings and uncertain motion without requiring third-party insertion of artificial landmarks into the environment. The de facto standard to localisation in recent times is the MCL method. The MCL method solves the standard localisation, or tracking problem, whereby the filter is able to localise a robot given that it already knows the approximate location of the robot. The method also solves the global localisation problem, where the filter is able to localise a robot without any information a priori. The theory behind the MCL method, and its variants are examined in Chapter 3 .

The simultaneous localisation and mapping problem and its various solutions are surveyed in this chapter, but are not developed further in this thesis. 


\section{Chapter 3}

\section{Theoretical Mapping and Localisation}

The importance of mapping, and localisation for mobile robotics is introduced in Chapter 1. A survey of popular mapping and localisation techniques is subsequently given in Chapter 2. In the current chapter, some interesting techniques to mapping and localisation are chosen, and their theoretical foundations are developed.

Consider an average mobile robot that may be deployed in a typical indoor structured environment. Such a robot has limited sensory capabilities to remain cost effective. It is also unable to use external localisation devices such as the Global Positioning System (GPS) for mapping and localisation since GPS is generally unavailable in an indoor environment. It is for such a robot that mapping and localisation techniques are fundamental for deployment. An example of such a robot is illustrated in Figure 3.1, adapted from [137]. As can be seen, the robot has a limited number of sensors and actuators. The robot is a differential drive robot with two drive wheels. The sensors shown are the bump sensors, and the laser range sensors. 
For the purposes of mapping and localisation, however, only the laser range sensors are of interest. Also important for mapping and localisation is the use of odometry information. Odometry is calculated according to commanded wheel speeds, and is not provided by a physical sensor that can be mounted on the robot; it is therefore not illustrated on the robot's diagram.

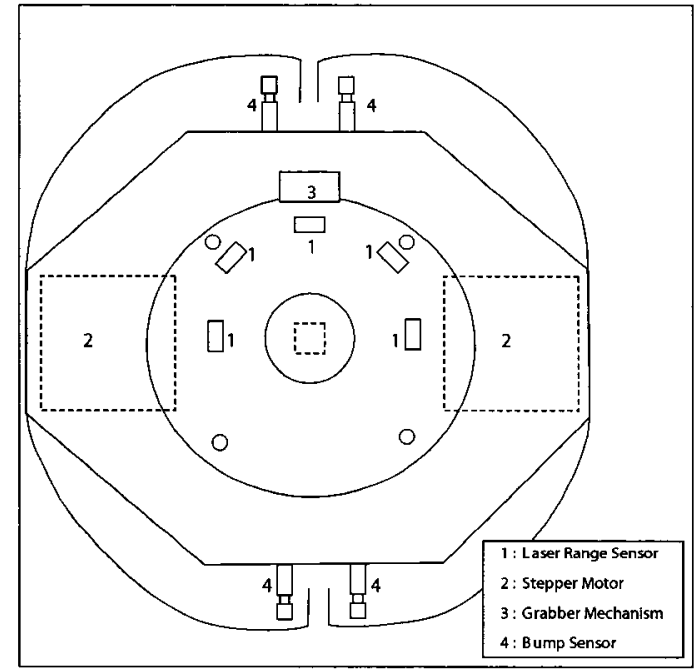

3.1a) Blueprint of Realistic Robot

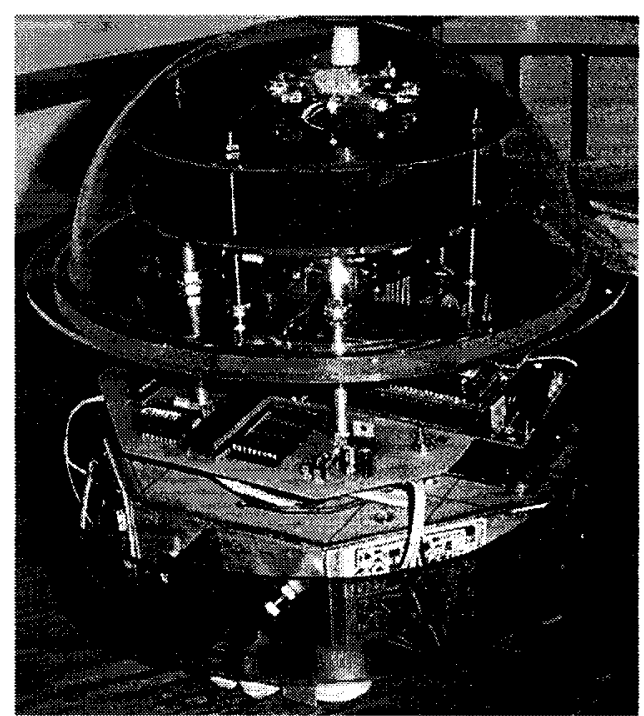

3.1b) Picture of Realistic Robot

Figure 3.1: Example of an Average Mobile Robot

Mapping and localisation techniques to be developed in this chapter are chosen with the example robot in mind, when it is deployed in an indoor structured environment.

The types of maps that are used in robotic mapping are described in Section 2.2. The two types of maps are grid based, and topological based, and they present complementary strengths and weaknesses. A grid based map, presented in Section 2.2.1, is chosen for many reasons:

- Explicit modeling of free-space allows for collision free path planning; 
- Cell size parameter of map allows for adjustable map resolution;

- Grid based map is usable with popular localisation techniques;

- Grid based map can be transformed into a combined map.

The grid based mapping method is developed in Section 3.1.

As mentioned in Section 2.3, Monte Carlo Localisation (MCL) and its variants are the de facto standard to localisation, used by many robots in recent time. MCL is popular due to its comparative effectiveness, and ease of implementation, thus, MCL and its variants are chosen for examination in Section 3.2.

\subsection{Mapping}

The map of the environment created by the robot is chosen to be based upon occupancy grids. From Section 2.2, grid based maps divide the environment into arrays of cells. Each cell has an associated numerical occupancy value, or probability of occupancy. These occupancy values need to be updated according to sensor observations, and various methods exist such as Bayesian theory, evidential theory, and fuzzy theory. The Bayesian method is by far the most popular method of updating the occupancy values, due to its mathematical basis, and early introduction by Elfes and Moravec. Though it is popular, there are some characteristics of the Bayesian method that are not desirable. The evidential methodology, on the other hand, appears to present the same advantages as the Bayesian method, but removes those undesirable characteristics.

This section first presents different interpretations of probability before developing the Bayesian and evidential methodology, which both prescribe the use of subjective 
probability. The two mapping methods are compared and contrasted, and the evidential method is ultimately the method of choice due to its advantages.

\subsubsection{Meaning of Probabilities}

There is ongoing debate as to the true meaning of, and applicability of, probability [115]; historical analysis into the development of probability theory as is known today is itself an involved topic $[113,114]$ with different popular interpretations during different time periods over the course of three centuries. The two major camps are of frequentism, and of subjectivism. Frequentists believe probability to be the chance of occurrence of an event determined by long term averages. Subjectivists believe probability to be a degree of belief, that may be purely qualitative, or be quantitatively warranted. One commonality between the two schools of thought is that both views share the same mathematical theory, such as Kolmogorov's Axioms.

Indeed, the standard interpretation of probability is a frequentist approach. As defined in standard probability texts [98], probability is a numerical value defined as $P(A) \approx \frac{n_{A}}{n}$, where $A$ is the event under observation, $n_{A}$ is the number of times the event occurs, and $n$ is the number of total times the experiment is performed. A classical definition of probability may also be given, where $P(A)=\frac{N_{A}}{N}$, where $N_{A}$ is the number of possible favourable occurrences of the event $A$, and $N$ is the total number of possible occurrences. The classical definition removes the need for experimentation. For some types of questions, however, it is more suited to adopt a subjective view to probability such as with the question "what is the probability that the Ottawa Senators will win against the Toronto Maple Leafs in the upcoming hockey game?" Such an experiment is not repeatable in a controlled manner, and to derive a probability of 0.5 using the classical definition would be incorrect. Such 
probability is best described to be the odds to a fair bet, derived using honest personal intuition. This is also known as a coherent bet [32], a bet to which a person will take either side of the bet knowing that they will break even in the long run. Therefore, the odds of the bet are set subjectively by a person, based on their honest knowledge of team skill, players involved, etc.. This is related to the concept of fair prices [113], studied by Pascal and Fermat before it was grouped with the idea of probability.

Though subjectively assigned probabilities may not reflect true probabilities one might observe following the frequentist methodology after large numbers of repeated experimental trials, it may be just as effective. Consider the classic experiment of a fair coin toss. Using the principle of insufficient reason, one would assert that the probability of getting a heads result is necessarily equal to the probability of getting a tails result at a value of 0.5. Experimental results would also seem to support that probability value [64]. Recent research [111], however, suggests that the coin toss is biased towards the side that the coin starts on before the toss. Thus, the assertion that heads is equally probable as tails is, in essence, a subjective probability value as to what odds are reasonable. Though it does not reflect the true probability, it is an effective value for a finite number of trials since there is no noticeable difference.

Subjective probability is sometimes known as the degree of belief in the occurrence of an event, and typically, this degree of belief is supported by some form of evidence. In mapping, the occupancy state may be estimated using the degree of belief that an area may or may not be occupied. Evidence that contributes to the degree of belief may come from a variety of sensor observations. Each sensor provides their own belief of map occupancy, and various sensors may agree, agree partially, or be completely contradictory to results of other sensors, since the sensors are not ideal. A method to merge these independent beliefs is needed to arrive at a single plausible 
result based on the evidence presented. Both Bayesian and Dempster-Shafer theory are viable methods for merging independent beliefs.

\subsubsection{Bayesian Theory}

Bayesian theory treats probability as a subjective degree of belief for the event of interest, and is so named for the use of Bayes' theorem for integrating new information to update the probability. Bayes' theorem (3.2) is derived by equating the two forms of the product rule (3.1), where $A$ and $B$ are events.

$$
\begin{gathered}
P(A B)=P(A \mid B) P(B) \\
P(A B)=P(B \mid A) P(A) \\
P(B \mid A)=\frac{P(A \mid B) P(B)}{P(A)}
\end{gathered}
$$

Although Bayes' theorem appears simple, it is very useful for integrating new information to determine the probability of an event. This is best illustrated with the following example.

Example: A doctor is interested in whether or not a patient has a flu $(\mathrm{F})$. The cold sickness has typical symptoms such as runny nose (RN), and high fever (HF). It 
is assumed that the doctor has access to the following probabilities:

$$
\begin{array}{rlrl}
P(F) & =0.2 & \text { Chance of the flu } \\
P(R N \mid F) & =0.15 \text { Chance of runny nose given that the patient has the flu } \\
P(H F \mid F) & =0.3 \quad \text { Chance of high fever given that the patient has the flu } \\
P(H F) & =0.1 \\
P(R N) & =0.1 \\
P(H F, R N) & =0.01 & \text { Chance of a high fever }
\end{array}
$$

(RN independent of $\mathrm{HF}$ )

The probabilities given are subjective probabilities prescribed by expert opinion (the doctor). For example, $P(F)=0.2$ means that for every hundred people, twenty will probably be sick from the flu. This may be due to the doctor's experience that there is a flu epidemic going around. Should the flu epidemic be contained, the doctor may revise $P(F)$ to a lower value.

Suppose the patient visits the doctor, and complains of a runny nose. To determine whether the patient has the flu, the doctor is interested in the quantity $P(F \mid R N)$ :

$$
\begin{aligned}
P(F \mid R N) & =\frac{P(R N \mid F) P(F)}{P(R N)} \\
& =\frac{(0.15)(0.2)}{0.1} \\
& =0.3 .
\end{aligned}
$$

With the single piece of information, there is a low probability that the patient has the flu. The runny nose symptom may correspond to other sicknesses the patient might have, like the common cold.

Suppose the patient complains of a high fever instead of a runny nose. The doctor 
is then interested in the quantity $P(F \mid H F)$ :

$$
\begin{aligned}
P(F \mid H F) & =\frac{P(H F \mid F) P(F)}{P(H F)} \\
& =\frac{(0.3)(0.2)}{0.1} \\
& =0.6 .
\end{aligned}
$$

With this piece of information, the probability of having a flu has increased, compared to the other symptom. The high fever symptom corresponds more closely to the flu, and the patient has a higher probability of having the flu.

If the patient visits the doctor reports that he suffers from both the runny nose and high fever symptom, the doctor can include the consideration of both symptoms to calculate the quantity $P(F \mid R N, H F)$, the chance of having the flu given that the patient has both symptoms:

$$
\begin{aligned}
P(F \mid R N, H F) & =\frac{P(H F \mid F, R N) P(F \mid R N)}{P(H F \mid R N)} \\
& =\frac{P(H F \mid F, R N)}{P(H F \mid R N)}\left(\frac{P(R N \mid F) P(F)}{P(R N)}\right) \quad \text { Sub in (3.3) } \\
& =\frac{P(H F \mid F)}{P(H F \mid R N)}\left(\frac{P(R N \mid F) P(F)}{P(R N)}\right) \quad \text { Since RN independent of HF } \\
& =\frac{P(H F \mid F) P(R N \mid F) P(F)}{P(H F, R N)} \\
& =\frac{P(H F \mid F) P(R N \mid F) P(F)}{P(H F) P(R N)} \\
& =\frac{(0.3)(0.15)(0.2)}{0.01} \\
& =0.9
\end{aligned}
$$

Now, both symptoms provide evidence to reinforce the probability that the patient has the flu, and the probability is much higher than with a single symptom.

This is the idea of the Bayesian method. Evidence that supports an event conditions the probability so that it becomes higher. Evidence that contradicts an event 
likewise reduces the probability so that it is lower.

\subsubsection{Bayesian Environment Mapping}

From the previous section, the Bayesian method provides a viable method from a probabilistic standpoint of merging evidence from different sources. For environment mapping, the event of interest is the occupancy of each grid cell that makes up a grid based map. The probability of each grid cell being occupied is conditioned upon sensory data that arrives periodically from the robot's sensors.

In a grid based map, assume that each cell is given a label $[i, j]$ indicating its position in the grid map. Let $O_{i, j}$ represent the event that the specific cell of the grid map is occupied, then $\overline{O_{i, j}}$ represents the event that the cell is free. These two events are mutually exclusive, and exhausts the sample space. For the remainder of the section, the $[i, j]$ labels will be omitted, as the results derived apply to all cells of the grid map.

The probability that the cell is occupied at time $t$ given a sensor reading $r$ is given in $(3.5)$.

$$
\begin{array}{rlr}
P\left(O_{t} \mid r_{t}\right) & =\frac{P\left(r_{t} \mid O_{t}\right) P\left(O_{t}\right)}{P\left(r_{t}\right)} & \text { Bayes rule } \\
& =\frac{P\left(r_{t} \mid O_{t}\right) P\left(O_{t}\right)}{P\left(r_{t} \mid O_{t}\right) P\left(O_{t}\right)+P\left(r_{t} \mid \overline{O_{t}}\right) P\left(\overline{O_{t}}\right)} & \text { theorem of total probability }
\end{array}
$$

The expression (3.5) is updated recursively by setting $P\left(O_{t}\right)=P\left(O_{t-1} \mid r_{t-1}\right)$.

With a Bayesian mapping framework, each cell maintains the probability $P(O)$ that it is occupied given sensor information. The inverse event $P(\bar{O})$ can be calculated by $P(\bar{O})=1-P(O)$. To model the initially unknown state of a cell when it is first initialised, the probability of occupancy for each cell is set to 0.5 . Given good sensory evidence about the occupancy of each grid cell, the cells will be updated over time 
to have a probability that approaches one for an occupied cell, and a probability that approaches zero for an empty cell. Though this framework works over time to

yield the correct results, it has an undesirable interpretation of probability, and of the unknown. With the use of $P(O)=P(\bar{O})=0.5$ to represent the unknown state of the grid cell, one cannot determine whether or not that value is due to contradicting sensor observations, or whether it is due to lack of sensor observations. From a linguistic point of view, this initialisation prescribes the idea that a cell's state has a $50 \%$ chance of being either occupied or empty, even without any sensor evidence to support either event. Thus, is not the best representation of the unknown.

An example of updating cell occupancy using the Bayesian method is given in Section 3.1.6.

\subsubsection{Dempster-Shafer Theory of Evidence}

In 1976, Shafer published ideas, terminology, and notations about the theory of belief functions in his book, A Mathematical Theory of Evidence [112]. Due to early work in the area by Dempster, and developments Shafer contributed in the area, this theory is often known as the Dempster-Shafer Theory of Evidence.

Dempster-Shafer theory is widely regarded as a statistical method of uncertain reasoning. It deals with weights of evidence, and numerical degrees of support for various propositions based on evidence. It also presents a method of combining distinct bodies of evidence supporting different propositions, leading to probable reasoning since no distribution is needed a priori.

With Dempster-Shafer theory, a Frame of Discernment $\theta$ (also known as the Universe of Discourse) is defined. The frame of discernment is comprised of all mutually exclusive propositions of interest, and is a finite discrete set. These basic propositions 
are called singleton propositions. With the frame of discernment, there are $2^{|\theta|}$ possible subsets, where $|\theta|$ is the cardinality of the set. Denote $2^{\theta}$ as the power set, the set that contains all the possible subsets. Each subset is also regarded as a possible proposition, and in this manner exhaustively proposes other more general propositions based on interactions between the singleton propositions. When a proposition exists in the power set $2^{\theta}$, it is said that the frame discerns that proposition.

Example: Let $\theta$ represent the set of all possible illnesses that can affect a person. Suppose there are only three diseases that can affect a person, and they are "the common cold" (CC), "skin cancer" (SC), and "breast cancer" (BC). Then,

$$
\theta=\{C C, S C, B C\}
$$

The power set $2^{\theta}$ that contains all possible sets of $\theta$ is

$$
2^{\theta}=\{\{C C, S C\},\{C C, B C\},\{S C, B C\},\{C C\},\{S C\},\{B C\}, \theta, \phi\},
$$

where $\phi$ is the empty set. Each set in $2^{\theta}$ is a proposition discerned by the frame of discernment, including singleton propositions as well as general propositions (sets with cardinality greater than one). The set $\{S C, B C\}$, for example, is a general proposition proposing that the person is sick with cancer, which may be either SC, or BC. General propositions may or may not have convenient interpretations, or may not even be valid combinations of singleton propositions. This depends on the definition of $\theta$.

\section{Representations of Chance}

There are multiple ways to represent chance in Dempster-Shafer formalism. 
A function $m$ is called a basic probability assignment (BPA) if it maps each proposition in the power set $2^{\theta}$ to a value such that it satisfies the following conditions:

$$
\begin{array}{ll}
m: 2^{\theta} \rightarrow[0,1] & \\
\text { where } & m(\phi)=0, \\
& \sum_{A \subseteq \theta} m(A)=1
\end{array}
$$

BPAs differ from standard probability assignments in that they assign probability values to general propositions in $2^{\theta}$, as opposed to just simply specific singleton propositions in $\theta$.

A function $\mathrm{Bel}$ is a belief function if it provides mappings for general propositions in $2^{\theta}$ such that they satisfy the following:

$$
\begin{aligned}
\operatorname{Bel}: 2^{\theta} \rightarrow[0,1] & \\
\text { where } & \operatorname{Bel}(\phi)=0 \\
& \operatorname{Bel}(\theta)=1 \\
& \operatorname{Bel}\left(A_{1} \cup \ldots \cup A_{n}\right) \geq \sum_{\substack{I \subseteq\{1 \ldots n\} \\
I \neq \phi}}(-1)^{|I|+1} \operatorname{Bel}\left(\bigcap_{i \in I} A_{i}\right)
\end{aligned}
$$

A belief function assigns the degree of belief in a proposition represented by a subset in the power set. A belief function is called a Bayesian belief function when the following conditions are satisfied:

$$
\begin{aligned}
& \operatorname{Bel}(\phi)=0 \\
& \operatorname{Bel}(\theta)=1 \\
& \operatorname{Bel}(A \cup B)=\operatorname{Bel}(A)+B e l(B), \forall A, B \subseteq \theta, \text { and } A \cap B=\phi \\
& m: \theta \rightarrow[0,1] \\
& \text { where } \quad \sum_{A \in \theta} m(A)=1 \\
& \qquad B e l(B)=\sum_{A \in B} m(A)
\end{aligned}
$$


This special case of the belief function is what makes Dempster-Shafer's theory of evidence appealing to many people; under these conditions, the Bayesian belief function produces the same results as Bayes rule, and occurs when complete knowledge of the chances involved are known. Dempster-Shafer theory is thus considered a generalisation of Bayesian theory, sharing the same theoretical mathematical framework. This idea is illustrated in Section 3.1.6 in the example comparing the Bayesian method to the Dempster-Shafer method with respect to a grid based mapping example.

BPAs and belief functions are equivalent in that each BPA corresponds to one belief function, and each belief function also corresponds to one BPA. They are related by the following equations:

$$
\begin{aligned}
& \operatorname{Bel}(A)=\sum_{B \subseteq A} m(B), \quad \forall A \subseteq \theta \\
& m(A)=\sum_{B \subseteq A}(-1)^{|A-B|} \operatorname{Bel}(B)
\end{aligned}
$$

BPAs assign the degree of belief for a general proposition $A \subseteq \theta$, however, that degree of belief may not yet be committed to a more specific proposition that exists in $B \subseteq 2^{A}$, a subset of $A$.

Two useful functions are the doubt function, $\operatorname{Dou}(A)=\operatorname{Bel}(\bar{A})$, and the upper probability function, $P^{*}(A)=1-\operatorname{Dou}(A)$. Doubt measures the degree of belief committed to other propositions that do not involve proposition $A$. The upper probability function measures the extent to which one does not doubt proposition $A$.

With these measures, it is expected that the true probability of a proposition $A$ will lie between $\left[\operatorname{Bel}(A), P^{*}(A)\right]$. Some refer to the lower bound $\operatorname{Bel}(A)$ as supportability, the degree of belief that directly supports a specific proposition $A$, and the higher bound $P^{*}(A)$ as plausibility, the degree of belief that does not directly contradict the proposition $A$. 


\section{Evidence Combination}

To be a viable theory for probable reasoning, there needs to be a method to merge distinct bodies of evidence. Though the original theory of evidence as originally presented by Shafer promoted the use of Dempster's rule of combination, other types of combinations are available as mentioned in [102]. Typical combination methods include:

1. Conjunctive integration

2. Disjunctive integration

3. Trade off integration

Conjunctive integration, represented by the operator $\oplus$, is the standard method of combining a new independent source of evidence. It is widely known as Dempster's rule of combination, and is specified as:

$$
\begin{aligned}
m_{1,2}(A) & =m_{1} \oplus m_{2}(A)=K \sum_{\substack{\forall B, C \in 2^{\theta} \\
B \cap C=A}} m_{1}(B) m_{2}(C), \\
K^{-1} & =1-\sum_{\substack{\forall B, C \in 2^{\theta} \\
B \cap C=\phi}} m_{1}(B) m_{2}(C) \\
m_{1,2}(\phi) & =m_{1} \oplus m_{2}(\phi)=0 .
\end{aligned}
$$

If $K^{-1}=0$, then the combination does not exist; $m_{\mathbf{1}}$ is said to be totally contradictory to $m_{2}$.

Disjunctive integration combines independent sources of evidence in the same manner as the union operation in set theory. It is specified as:

$$
m_{1,2}(C)=\sum_{A \cup B=C} m_{1}(A) \cdot m_{2}(B) .
$$


Introduced by Dubois and Prade [39], this method of evidence combination does not introduce any conflict, and does not require normalisation like in conjunctive integration. This approach may, however, lead to less precise reasoning since it does not deal with conflicting evidence.

Trade off integration combines $n$ independent sources of evidence by giving different weights $\alpha$ to different sources. The different weights are to be determined according to the reliability of the different sources so that highly conflicting evidence can be resolved. This alternative integration method is simply a weighted sum, and was proposed by Shafer in his original presentation of the theory of evidence [112] as he recognized the fallacy of Dempster's rule of combination amidst highly conflicting bodies of evidence. This integration method is specified by:

$$
\operatorname{Bel}(A)=\sum_{i=1}^{n} \alpha_{i} \operatorname{Bel}\left(A_{i}\right)
$$

where

$$
\sum_{i=1}^{n} \alpha_{i}=1, \quad \alpha_{1}, \ldots, \alpha_{n} \geq 0 .
$$

Example: (Adapted from [138]) A machine malfunctions that has three major components. A technician examines the machine and indicates that there is a $99 \%$ chance that component $A$ caused the failure, and there is a $1 \%$ chance that component $B$ caused the failure. Meanwhile, a second technician agrees that there is a $1 \%$ chance that component $B$ is the cause of the failure, but expresses his belief that there is a $99 \%$ chance component $C$ caused the failure. This scenario is summarised in Table 3.1.

Dempster-Shafer theory is used in this example to analyse which component is most likely to have caused the failure. The frame of discernment is $\theta=\{A, B, C\}$, and the power set is $2^{\theta}=\{A, B, C,\{A, B\},\{A, C\},\{B, C\}, \theta, \phi\}$. The use of conjunctive, 
disjunctive, and trade off data integration is examined in this example. For the purposes of both conjunctive and disjunctive data integration, no further information is required from the scenario, since conjunctive integration treats both technicians as equally reliable, while disjunctive integration analyses the situation from the point of view that at least one technician is reliable. Trade off integration, however, requires the weight of each technician's analysis. This implies that there is a third party that assigns weights $\alpha_{1}, \alpha_{2}$ to each technician's analysis, perhaps based on the technicians' experience. Let $\alpha_{1}=0.1$, and $\alpha_{2}=0.9$, due to the fact the first technician is a newhire, while the second technician has been on the job for many years. Intermediary calculations useful for conjunctive and disjunctive data integration are summarised in Table 3.2. The table contains $m_{1}(\cdot) m_{2}(\cdot)$ for the different propositions involved.

Table 3.1: Dempster-Shafer Example: Summary of Technicians' Opinions

\begin{tabular}{|c||c|c|c|c|c|c|c|c|}
\hline & $m(A)$ & $m(B)$ & $m(C)$ & $m(A B)$ & $m(A C)$ & $m(B C)$ & $m(\theta)$ & $m(\phi)$ \\
\hline \hline First opinion $\left(\alpha_{1}=0.1\right)$ & 0.99 & 0.01 & 0 & 0 & 0 & 0 & 0 & 0 \\
\hline Second opinion $\left(\alpha_{2}=0.9\right)$ & 0 & 0.01 & 0.99 & 0 & 0 & 0 & 0 & 0 \\
\hline
\end{tabular}

Table 3.2: Dempster-Shafer Example: Table of Calculations

\begin{tabular}{|c||c|c|c|}
\hline & $m_{2}(A)=0$ & $m_{2}(B)=0.01$ & $m_{2}(C)=0.99$ \\
\hline \hline$m_{1}(A)=0.99$ & 0 & 0.0099 & 0.9801 \\
\hline$m_{1}(B)=0.01$ & 0 & 0.0001 & 0.0099 \\
\hline$m_{1}(C)=0$ & 0 & 0 & 0 \\
\hline \hline
\end{tabular}

Non-singleton propositions are not included in this table (their values are 0 )

Using (3.6), Dempster's rule of combination is used to perform conjunctive integration on the two data sources. 
- Calculate $K^{-1}$ which is used for all other calculations

$$
\begin{aligned}
K^{-1}= & 1-m_{1}(A) m_{2}(B)-m_{1}(A) m_{2}(C)-m_{1}(B) m_{2}(C) \\
& -m_{1}(A) m_{2}(B C)-m_{1}(B) m_{2}(A C)-m_{1}(C) m_{2}(A B) \\
= & 1-0.0099-0.9801-0.0099 \\
= & 0.0001
\end{aligned}
$$

- Calculate $m_{1} \oplus m_{2}(A)$

$$
\begin{aligned}
m_{1} \oplus m_{2}(A)= & K\left(m_{1}(A) m_{2}(A)+m_{1}(A) m_{2}(A B)+m_{1}(A) m_{2}(A C)\right. \\
& \left.+m_{1}(A) m_{2}(A B C)\right) \\
= & K(0) \\
= & 0
\end{aligned}
$$

- Calculate $m_{1} \oplus m_{2}(B)$

$$
\begin{aligned}
m_{1} \oplus m_{2}(B)= & K\left(m_{1}(B) m_{2}(B)+m_{1}(B) m_{2}(A B)+m_{1}(B) m_{2}(B C)\right. \\
& \left.+m_{1}(B) m_{2}(A B C)\right) \\
= & K(0.0001) \\
= & 1.0
\end{aligned}
$$

- Calculate $m_{1} \oplus m_{2}(C)$

$$
\begin{aligned}
m_{1} \oplus m_{2}(C)= & K\left(m_{1}(C) m_{2}(C)+m_{1}(C) m_{2}(B C)+m_{1}(C) m_{2}(A C)\right. \\
& \left.+m_{1}(C) m_{2}(A B C)\right) \\
= & K(0) \\
= & 0
\end{aligned}
$$

- Non-singleton propositions are not expanded here, ie: $m_{1} \oplus m_{2}(A B)$, since they will all yield a value of zero

As can be seen from this example, $K^{-1} \approx 0$, which indicates that the beliefs expressed by the two technicians are almost flatly contradictory with respect to each other. This 
example given by Zadeh [138] accentuates the fallacy of Dempster's Rule when beliefs are highly contradictory. In this case, although both technicians express low support for component $B$ causing the machine failure, it appears certain that component $B$ is the cause of the failure after using Dempster's Rule as the method of data integration, since $m_{1} \oplus m_{2}(B)=1.0$.

Using (3.7), disjunctive data integration is applied to combine the two data sources. Table 3.3 summarises the data integration. As can be seen, disjunctive data integration works better than conjunctive data integration in this example; after data integration, it can be seen that either component $A$ or component $C$ may be broken, in accordance to the opinions of the two technicians. An example calculation for $m_{1} m_{2}(A C)$, that component $A$ or $C$ is broken, is shown below:

$$
\begin{aligned}
m_{1} m_{2}(A C) & =m_{1}(A) m_{2}(C) \\
& =0.9801
\end{aligned}
$$

Table 3.3: Dempster-Shafer Example: Table of Results for Disjunctive Data Integration

\begin{tabular}{|l||c|}
\hline Component A broken, $m_{1} m_{2}(A)$ & 0 \\
\hline Component B broken, $m_{1} m_{2}(B)$ & 0.0001 \\
\hline Component C broken, $m_{1} m_{2}(C)$ & 0 \\
\hline Component A or B broken, $m_{1} m_{2}(A B)$ & 0.0099 \\
\hline Component A or C broken, $m_{1} m_{2}(A C)$ & 0.9801 \\
\hline Component B or C broken, $m_{1} m_{2}(B C)$ & 0.0099 \\
\hline Component A or B or C broken, $m_{1} m_{2}(A B C)$ & 0 \\
\hline
\end{tabular}

To use trade off integration, the weight values $\alpha_{1}, \alpha_{2}$ are used. The weights satisfy (3.9), and (3.8) is used to calculate the integrated BPA. Table 3.4 summarises the integrated BPA. As can be seen, this integration method simply considers the more experienced technician's opinion more heavily than the first, less experienced technician. 
Table 3.4: Dempster-Shafer Example: Table of Results for Trade Off Data Integration

\begin{tabular}{|c||c|}
\hline Component A broken, $m_{1} m_{2}(A)$ & $\alpha_{1} m_{1}(A)+\alpha_{2} m_{2}(A)=0.099$ \\
\hline Component B broken, $m_{1} m_{2}(B)$ & $\alpha_{1} m_{1}(B)+\alpha_{2} m_{2}(B)=0.01$ \\
\hline Component C broken, $m_{1} m_{2}(C)$ & $\alpha_{1} m_{1}(C)+\alpha_{2} m_{2}(C)=0.891$ \\
\hline \hline
\end{tabular}

Non-singleton propositions are not included since they are zero. ie: Component $\mathrm{A}$ or $\mathrm{B}$ broken, $m_{1} m_{2}(A B)=0$

As can be seen, Dempster-Shafer not only models ignorance, but also allows general propositions to be assigned probability values. In the previous example, suppose the newly hired technician could determine with an $80 \%$ chance that either component $A$ or component $B$ was broken, but could not narrow his or her hypothesis to either component. The Dempster-Shafer framework allows this belief to be expressed and integrated by assigning the probability to $m_{1}(A B)=0.8$, rather than the singleton propositions. This is not possible in other frameworks, such as the Bayesian framework.

\subsubsection{Evidential Environment Mapping}

Evidential environment mapping makes use of Dempster-Shafer theory described in the previous section to combine independent sources of evidence from sensors to provide the numerical occupancy value.

As described in Section 3.1.3, although the Bayesian method is a viable method to merge different sources of evidence, it does not have a good mechanism to represent the unknown. As well, all probabilities are presumed to be known. That is to say, if the sensor data provides a 0.7 numerical support for a grid cell to be occupied, it is implicitly providing 0.3 numerical support for the inverse event, that the grid cell is empty.

As such, the greatest advantage with using an evidential framework is the ability 
to model the unknown. With the evidential method, the map encodes within it many interesting measures such as the degree of belief for occupancy, emptiness, and the lack of knowledge. These measures allow distinction between contradictory sensor observations, and the lack of information which are possible in a practical environment with noisy and error prone sensors.

Using Dempster-Shafer theory to model the occupancy of a grid cell, following [97], a frame of discernment is defined for each cell. The two relevant propositions that belong in the frame are that the cell is occupied, and that the cell is empty. The frame of discernment, and its power set are therefore

$$
\begin{aligned}
& \theta=\{O, E\} \\
& 2^{\theta}=\{\phi, O, E,\{O, E\}\}
\end{aligned}
$$

Each set in the power set $2^{\theta}$ is a general proposition in the domain which also expresses the interaction between singleton propositions in the frame of discernment. $O$ indicates occupancy; $E$ indicates emptiness; $\{O, E\}$ indicates uncommitted belief for any proposition; $\phi$ indicates that no other proposition is applicable to the cell. Due to the small size of the frame of discernment, the power set does not present any interaction between the singleton propositions.

The following BPA is used to initialise the state of the grid cells:

$$
\begin{aligned}
m(O) & =0 \\
m(E) & =0 \\
m(O, E) & =1 \\
m(\phi) & =0
\end{aligned}
$$

As discussed earlier, conjunctive integration does not give intuitive results when presented with highly contradictory evidence in certain cases. This, however, is not 
expected to occur in its application towards mapping. Therefore, conjunctive integration is used, and Dempster-Shafer theory as originally defined in [112] is used.

The various measures for each proposition give useful information about that proposition. To determine the degree of belief for the occupancy of a cell, the support $\operatorname{Bel}(O)$, and the plausibility $P^{*}(O)$ can be calculated. For a freshly initialised cell, it is calculated that

$$
\begin{aligned}
& \operatorname{Bel}(O)=0 \\
& P^{*}(O)=1 .
\end{aligned}
$$

As can be seen, there no reason to believe the cell is occupied with the $\operatorname{Bel}(O)$ measure, but there is also no evidence to say the cell is not occupied with the plausibility $P^{*}(O)$ measure. The same can be done for $\operatorname{Bel}(E)$ and $P^{*}(E)$. Compared to the initialisation for the Bayesian mapping method, the evidential method far better represents the unknown.

The evidential framework was therefore chosen for the mapping operation for its advantages. An example is given in Section 3.1.6.

\subsubsection{Brief Comparison of Bayesian and Evidential Mapping}

In the sections preceding, both the Bayesian method and the evidential method are presented as viable methods to merge separate sources of evidences, from robot sensors in particular, to arrive at a single belief of the occupancy of map grid cells. The evidential method is promoted as a better method due to its ability to model the unknown. In this section, concrete examples of mapping using the Bayesian method and the evidential method are given to contrast and compare the methods. 


\section{Bayesian Example}

This example uses the Bayesian formulation to mapping given in Section 3.1.3. $P(O)$ gives the probability that a cell is occupied, and the inverse event $P(\bar{O})=1-P(O)$ gives the probability that a cell is empty. The initial state of all cells is initialised by setting $P\left(O_{0}\right)=0.5$. Given sensor readings $r_{t},(3.5)$ is used to recursively condition the occupancy of the cell, reproduced below:

$$
\begin{aligned}
& P\left(O_{t} \mid r_{t}\right)=\frac{P\left(r_{t} \mid O_{t}\right) P\left(O_{t}\right)}{P\left(r_{t}\right)} \quad \text { Bayes rule } \\
& =\frac{P\left(r_{t} \mid O_{t}\right) P\left(O_{t}\right)}{P\left(r_{t} \mid O_{t}\right) P\left(O_{t}\right)+P\left(r_{t} \mid \overline{O_{t}}\right) P\left(\overline{O_{t}}\right)} \text { theorem of total probability }
\end{aligned}
$$

The expression is updated recursively by setting $P\left(O_{t}\right)=P\left(O_{t-1} \mid r_{t-1}\right)$.

Suppose a series of sensor data arrive to update the state of the grid cell, and assume that $P\left(r_{i} \mid O_{i}\right)=0.7$ therefore implying that $P\left(r_{i} \mid \overline{O_{i}}\right)=1-P\left(r_{i} \mid O_{i}\right)=0.3$ for $i=0 \ldots 3$. These are three separate sensor measurements that yield the same observations, unrealistic, but they are sufficient for the sensor data merge example. Using (3.5), the results of the sensor data merge are summarised in Table 3.5.

\section{Evidential Example}

This example uses the Dempster-Shafer approach to mapping developed in Section 3.1.5. In the formulation, the Frame of Discernment is $\theta=\{O, E\}$, where $O$ represents cell occupancy, and $E$ represents cell emptiness, and the corresponding power set is $2^{\theta}=\{O, E,\{O, E\}, \phi\}$. Conjunctive data integration (3.6) is used to integrate sensor data in this example.

Initially, the state of the cell is again unknown. In the Dempster-Shafer approach, 
the initial belief for the different states of the cell is:

$$
\begin{aligned}
\operatorname{Bel}(O) & =0 \\
\operatorname{Bel}(E) & =0 \\
\operatorname{Bel}(O E) & =1 \\
\operatorname{Bel}(\phi) & =0
\end{aligned}
$$

Suppose that a series of sensor data arrives to update the state of the grid cell like in the Bayesian example. The sensor data is interpreted to provide the following BPA:

$$
\begin{aligned}
m(O) & =0.7 \\
m(E) & =0.3 \\
m(O E) & =0 \\
m(\phi) & =0 .
\end{aligned}
$$

Integrating the sensor data, the results of the sensor data merge are summarised in Table 3.5.

Table 3.5: Results for Sensor Data Merge Using Bayesian and Dempster-Shafer Methods

\begin{tabular}{|c||c||c|c|c|}
\hline \multicolumn{1}{|c||}{ Time $(t)$} & \multirow{2}{*}{ Bayesian Merge } & \multicolumn{3}{c|}{ Dempster-Shafer Theory } \\
& & $m(O)$ & $m(E)$ & $m(O E)$ \\
\hline 0 & 0.5 & 0 & 0 & 1 \\
\hline 1 & 0.7 & 0.7 & 0.3 & 0 \\
\hline 2 & 0.8448 & 0.8448 & 0.1552 & 0 \\
\hline 3 & 0.9270 & 0.9270 & 0.0730 & 0 \\
\hline \multicolumn{5}{|c|}{$\vdots$} \\
\hline
\end{tabular}

\section{Comparison of Bayesian and Evidential Results}

With the results of the data merge from both the Bayesian method and DempsterShafer method in Table 3.5, it can be seen that the two approaches yield the same 
results as briefly mentioned in Section 3.1.4. However, as previously discussed in Section 3.1.5, it is already clear that the Dempster-Shafer approach is more expressive in its representation of the unknown. Dempster-Shafer method explicitly models ignorance of the state of the cell due to lack of sensor evidence. Also, sensor data indicating $70 \%$ chance of cell occupancy with the Bayesian method implies that the sensor is also saying that there is a $30 \%$ chance of cell emptiness. It is reasonable that this implicit assumption is true, but the evidential approach provides a different viewpoint. The sensor data prescribes 0.7 support, or evidence, that the cell is occupied. The sensor, however, does not prescribe any explicit support that the cell is empty! This ignorance of cell emptiness is expressible through the following BPA, hereby called occupancy support:

$$
\begin{aligned}
m(O) & =0.7 \\
m(E) & =0 \\
m(O E) & =0.3 \\
m(\phi) & =0
\end{aligned}
$$

With this interpretation of sensor data, a similar BPA must be defined to explicitly provide support for cell emptiness, hereby called emptiness support:

$$
\begin{aligned}
m(O) & =0 \\
m(E) & =0.2 \\
m(O E) & =0.8 \\
m(\phi) & =0
\end{aligned}
$$

The revised Dempster-Shafer examples in the next section illustrates characteristics of using the differently defined BPAs. 


\section{Revised Evidential Examples}

There are two examples in this section that illustrate various characteristics of how the evidential approach behaves under different conditions.

Example 1: Suppose the initial belief of the cell state is initialised using the BPA in (3.10), and 19 pieces of sensor data arrive to provide empty support (ES), represented with the BPA in (3.12). A 20th piece of sensor data follows soon after to provide occupancy support (OS), represented with the BPA in (3.11). Table 3.6 summarises the results of the sensor data merge using conjunctive integration shown in (3.6).

As seen in the results, by time 19, the large amount of ES causes the belief that the cell is empty to be a high value of 0.9885 . Because there has been no evidence to contradict the claim that the cell is empty, the plausibility measure is $P^{*}(E)=1$.

In contrast, at time $19, \operatorname{Bel}(O)=0$ indicates that there is no support indicating that the cell is occupied, and the low plausibility measure indicates that there is little chance that the cell is occupied due to the large amount of contradictory evidence.

With the 20th piece of sensor evidence, the measures still indicate that there is overwhelming support for the cell to be empty, but the measures for the occupancy of the cell is revised in a manner that indicates there is some support for occupancy. This shows that spurious sensor measurements do not affect the state of the cell, but if there is further support for the cell to be occupied, the state of the cell may be updated to reflect the change.

Example 2: In previous examples, the initial belief of the cell state is initialised 
Table 3.6: Results for Data Merge Using Revised Dempster-Shafer Methods

\begin{tabular}{|c||c|c|c|c|c|}
\hline Time $(t)$ & $m(O)=\operatorname{Dou}(E)$ & $m(E)=\operatorname{Dou}(O)$ & $m(O E)$ & $P^{*}(O)$ & $P^{*}(E)$ \\
\hline \hline 0 & 0 & 0 & 1 & 1 & 1 \\
\hline 1 & 0 & 0.2 & 0.8 & 0.8 & 1 \\
\hline 2 & 0 & 0.36 & 0.64 & 0.64 & 1 \\
\hline 3 & 0 & 0.488 & 0.512 & 0.512 & 1 \\
\hline 4 & 0 & 0.5904 & 0.4096 & 0.4096 & 1 \\
\hline 5 & 0 & 0.6723 & 0.3277 & 0.3277 & 1 \\
\hline \multicolumn{7}{|c|}{} \\
\hline 19 & 0 & 0.9885 & 0.0115 & 0.0115 & 1 \\
\hline 20 & 0.0262 & 0.9625 & 0.0113 & 0.0375 & 0.9738 \\
\hline
\end{tabular}

such that $m(O E) \neq 0$. Suppose the belief of the cell state is initialised to

$$
\begin{aligned}
m(O) & =0.8 \\
m(E) & =0.2 \\
m(O E) & =0 \\
m(\phi) & =0
\end{aligned}
$$

and a piece of sensor data arrives to provide ES. Table 3.7 summarises the results of the sensor data merge. As can be seen, Dempster-Shafer theory adjusts the support accordingly.

Table 3.7: Results for Data Merge Using Revised Dempster-Shafer Methods (Case 2)

\begin{tabular}{|c||c|c|c|c|c|}
\hline Time $(t)$ & $m(O)=\operatorname{Dou}(E)$ & $m(E)=\operatorname{Dou}(O)$ & $m(O E)$ & $P^{*}(O)$ & $P^{*}(E)$ \\
\hline \hline 0 & 0.8 & 0.2 & 0 & 0.8 & 0.2 \\
\hline 1 & 0.7619 & 0.2381 & 0 & 0.7619 & 0.2381 \\
\hline
\end{tabular}

\subsection{Localisation}

The Monte Carlo Localisation (MCL) method and its variants introduced in Section 2.3.3 are examined in this section. As mentioned previously, MCL is a sample 
based implementation of the standard Bayesian recursive equation that underlies all Bayesian filters. With $s_{t}$ being the state of the robot, $o_{t}$ a sensor observation, and $a_{t}$ an action by the robot, the Bayesian recursive equation (2.6) that estimates the state of the robot is reproduced below:

$$
\operatorname{Bel}\left(s_{t}\right)=\eta P\left(o_{t} \mid s_{t}\right) \int P\left(s_{t} \mid a_{t-1} s_{t-1}\right) \operatorname{Bel}\left(s_{t-1}\right) d s_{t-1}
$$

The integral in (2.6), in its most general sense, does not have an analytic closed form solution. The multidimensional, nonlinear, continuous nature of the quantities involved also makes the recursive equation impossible to compute. Implementing any naïve method of calculating the equation will definitely fail as a viable solution for mobile robotics, where computation power is at a premium.

In MCL, the continous nature of the probability density functions (pdf) involved are approximated with samples, also known as particles. This changes the continous nature of (2.6) into a discrete nature, and makes the recursive equation a tractable one to compute.

The evolution of particle filters, the main component of MCL, is discussed in the following. The development of particle filters involves ideal Monte Carlo sampling, importance sampling, sequential importance sampling, and sampling importance resampling.

\subsubsection{Ideal Monte Carlo Sampling}

At the heart of particle filters is the application of Monte Carlo approximation. The robot state estimation problem is formulated such that the calculus that is difficult to solve analytically can be approximated by algebraic summations. The appropriateness of using Monte Carlo approximation to solve such calculations is demonstrable 
through ideal Monte Carlo sampling. The general Monte Carlo method of approximation is first described. A concrete example of using Monte Carlo approximation to solve a single variable integration is then detailed, followed by a description of how to use Monte Carlo approximation for robot localisation.

Given a random variable $X$ that has a probability density function $f_{X}(x)$, the expected value of a function $g$ of $X$ is

$$
E(g(X))=\int g(x) \cdot f_{X}(x) d x
$$

Assuming that $N$ independent and identically distributed (i.i.d.) samples are drawable from $X,\left(x_{1}, x_{2}, \ldots, x_{N}\right)$, then an empirical approximation to the probability density function is specified by

$$
f_{N}(x)=\frac{1}{N} \sum_{i=1}^{N} \delta\left(x-x_{i}\right)
$$

where $\delta\left(x-x_{i}\right)$ is the dirac delta distribution.

Note that the approximate density function in (3.13) is not to be confused with a sampling function, since the samples $x_{i}$ are distributed according to $X$. Given the Gaussian distribution depicted in Figure 3.2a, the rest of the figures in Figure 3.2 show empirical density approximations using varying numbers of samples. It can be seen that an increased number of samples produces a better approximation.

With the empirical probability density function approximation specified in (3.13), the sample average, also known as the Monte Carlo estimate, is sufficient to estimate $E[g(X)]$. The Monte Carlo estimator is a form of the Monte Carlo estimate that is a function of the random variable $X$. 


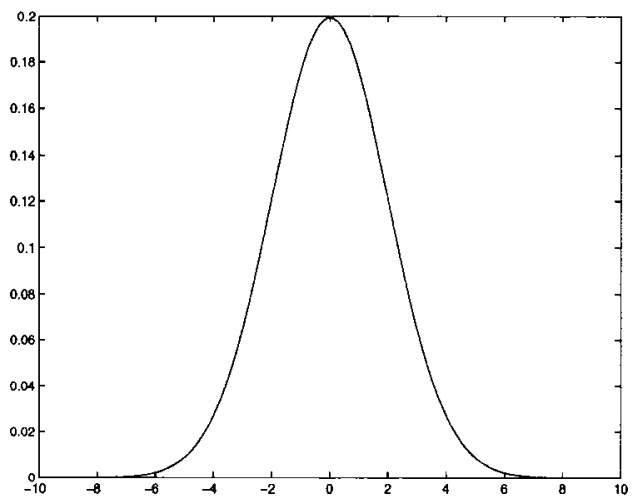

3.2a) Gaussian Distribution

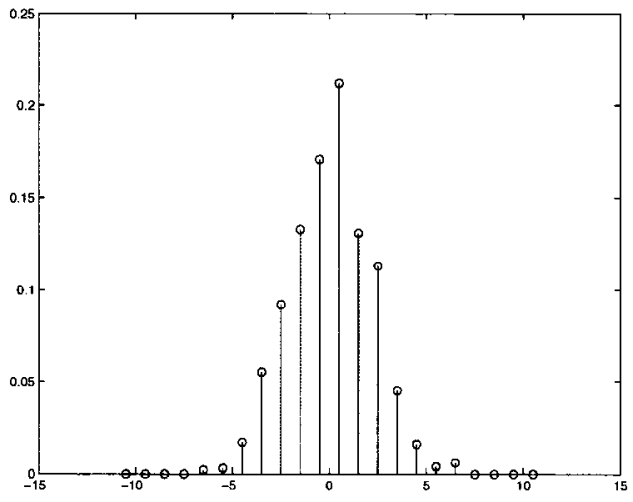

3.2c) 1000 Sample Approximation (0.5 bin size)

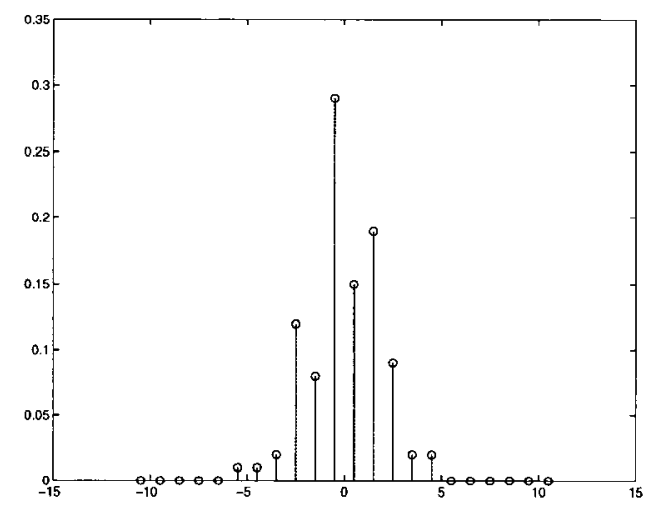

3.2b) 100 Sample Approximation ( 0.5 bin size)

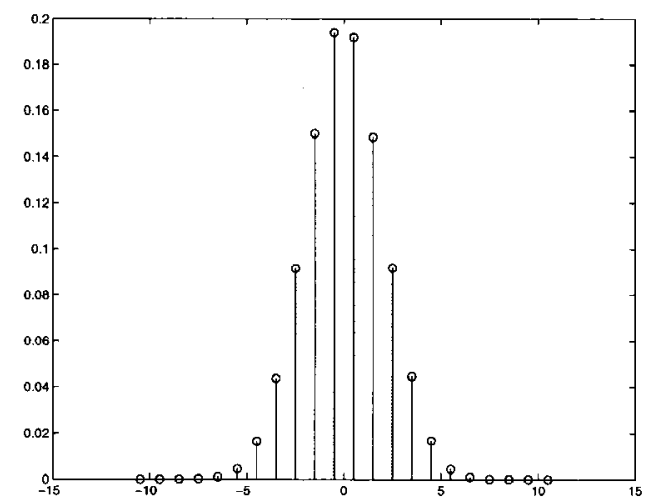

3.2d) 10000 Sample Approximation (0.5 bin size)

Figure 3.2: Effect of Sampling Probability Distribution

$$
\begin{aligned}
\tilde{g}_{N}(x)=\frac{1}{N} \sum_{i=1}^{N} g\left(x_{i}\right) & \text { Monte Carlo Estimate } \\
\tilde{g}_{N}(X)=\frac{1}{N} \sum_{i=1}^{N} g(X) & \text { Monte Carlo Estimator }
\end{aligned}
$$

Both the Monte Carlo estimate and estimator are sufficient to estimate $E[g(X)]$ 
since

$$
\begin{aligned}
E\left[\tilde{g}_{N}(X)\right] & =E\left[\frac{1}{N} \sum_{i=1}^{N} g(X)\right] \\
& =\frac{1}{N} \sum_{i=1}^{N} E[g(X)] \\
& =E[g(X)]
\end{aligned}
$$

Due to the weak law of large numbers, $\lim _{N \rightarrow \infty} P\left[\left|\tilde{g}_{N}(X)-\mu\right|<\epsilon\right]=1$; for a sufficient number of samples $N$, the estimator will have a mean value that is within an $\epsilon$ distance to the true mean value $\mu$. In this situation, the strong law of large numbers also applies such that $P\left[\lim _{N \rightarrow \infty} f_{N}(X)=\mu\right]=1$. As the number of samples approaches infinity, the sample mean will approach the true mean value $\mu$. With these characteristics, the estimator is consistent and unbiased.

The idea of using repeated random sampling from the probability density function to solve functions in a numerical manner is best seen in an example. Given the simple integral function in (3.14), it is possible to reformulate the function so that it can be solved using Monte Carlo approximation. This is done by transforming the function such that it is a function $g$ of a random variable $X$ (3.15). Since the function of interest is a simple bounded integral, the probability density function for $X$ is chosen to be a uniform distribution from 0 to 1 . The expectation shown in (3.16) can then be approximated using $N$ random samples distributed according to the uniform distribution, like in (3.17). The approximated expectation is then the estimated solution to the function.

$$
f(x)=\int_{0}^{1} e^{-x} d x
$$




$$
\begin{aligned}
g(X)= & e^{-X} \\
E[f(x)] & =\int_{-\infty}^{\infty} f(x) p(x) d x, \quad \text { where } p(x) \sim U(0,1) \\
E[f(x)] & \approx \frac{1}{N} \sum_{i=0}^{N} e^{-x} \delta\left(x-x^{(i)}\right) \\
& \approx \frac{1}{N} \sum_{i=0}^{N} e^{-x^{(i)}}
\end{aligned}
$$

The approximate expectation was evaluated using Monte Carlo sampling with different numbers of samples, and the results are shown in Table 3.8 . It can be seen that the estimate gets increasingly accurate as the number of samples used is increased.

Table 3.8: Summary of Monte Carlo Approximations to Simple Integral

\begin{tabular}{|c|c|c|}
\hline Samples & Analytic Solution & Estimated Solution \\
\hline \hline 10 & \multirow{3}{*}{0.6321} & 0.6961 \\
\cline { 1 - 1 } 100 & & 0.6731 \\
& & 0.6366 \\
\hline 1,000 & & 0.6320 \\
\hline 10,000 & &
\end{tabular}

In applying the Monte Carlo sampling method to robot localisation, the distribution of interest is the joint density function $\operatorname{Bel}\left(s_{t}\right)$, or $p\left(s_{t} \mid d_{0 \ldots t}\right)$ (instead of $f_{X}(x)$ ). In essence, samples are to be drawn from possible states of the robot in the environment, and the actual state of the robot is desired. It is, however, not easy to sample from the posterior distribution due to reasons such as:

- distribution is known only approximately from recursive calculations, and usually only up to a proportionality constant

- distribution is a non standard distribution, and is difficult to generate i.i.d. samples from a simple equation 
- distribution is a multivariate quantity

\subsubsection{Importance Sampling}

Importance sampling attempts to address the issues with ideal Monte Carlo sampling and is described in greater detail in [33, 38, 47]. Since the sample space is created from a multivariate quantity, the sample space is necessarily large for a typical environment a robot might be deployed within. The key idea of importance sampling is to sample from important areas of the sample space, so that less computation is wasted in areas of the sample space that contain unlikely locations of the robot. In this section, a general treatment of importance sampling is given, along with a simple example illustrating the usefulness of importance sampling to alleviate computational requirements. Then, the application of importance sampling with respect to robot localisation is discussed.

Importance sampling assumes that an alternative distribution (importance function) $\pi\left(x_{1 \ldots t} \mid y_{0 \ldots t}\right)$ is available, and is related to the distribution of interest, $p\left(x_{1 \ldots t} \mid y_{0 \ldots t}\right)$. The importance function $\pi(\cdot)$ is expected to produce likely samples for $p(\cdot)$, and be easier to sample from. Mathematically, importance sampling using the alternative distribution $\pi(\cdot)$ is equivalent to ideal Monte Carlo sampling as seen in (3.18).

$$
\begin{aligned}
E\left[f\left(x_{0 \ldots t}\right)\right] & =\int f\left(x_{0 \ldots t}\right) p\left(x_{0 \ldots t} \mid y_{0 \ldots t}\right) d x_{0 \ldots t} \\
& =\int f\left(x_{0 \ldots t}\right) p\left(x_{0 \ldots t} \mid y_{0 \ldots t}\right) \frac{\pi\left(x_{0 \ldots t} \mid y_{0 \ldots t}\right)}{\pi\left(x_{0 \ldots t} \mid y_{0 \ldots t}\right)} d x_{0 \ldots t} \\
& =\int f\left(x_{0 \ldots t}\right) \frac{p\left(x_{0 \ldots t} \mid y_{0 \ldots t}\right)}{\pi\left(x_{0 \ldots t} \mid y_{0 \ldots t}\right)} \pi\left(x_{0 \ldots t} \mid y_{0 \ldots t}\right) d x_{0 \ldots t} \\
& =\int f\left(x_{0 \ldots t}\right) w\left(x_{0 \ldots t}\right) \pi\left(x_{0 \ldots t} \mid y_{0 \ldots t}\right) d x_{0 \ldots t}, \quad \text { where } w\left(x_{0 \ldots t}\right)=\frac{p\left(x_{0 \ldots t} \mid y_{0 \ldots t}\right)}{\pi\left(x_{0 \ldots t} \mid y_{0 . . t}\right)}
\end{aligned}
$$


With importance sampling, the samples are now sampled from $\pi(\cdot)$. Due to the fact the samples being drawn are from a different distribution, those samples are weighted differently according to the $w(\cdot)$ factor according to their importance in the original distribution.

Suppose i.i.d. samples $x_{0 \ldots t}^{(i)}=\left\{x_{0 \ldots t}^{(1)}, \ldots, x_{0 . . t}^{(N)}\right\}$ are simulated from $\pi\left(x_{0 \ldots t} \mid y_{0 \ldots t}\right)$, then the approximation $\Pi_{N}\left(x_{0 \ldots t} \mid y_{0 . . t}\right)=\frac{1}{N} \sum_{i=1}^{N} \delta\left(x_{0 \ldots t}-x_{0 \ldots t}^{(i)}\right)$ can be substituted into (3.18) to yield

$$
\begin{aligned}
E\left[f\left(x_{0 . \ldots t}\right)\right] & =\int f\left(x_{0 \ldots t}\right) w\left(x_{0 \ldots t}\right) \Pi_{N}\left(x_{0 \ldots t} \mid y_{0 \ldots t}\right) d x_{0 \ldots t} \\
& \approx \frac{1}{N} \sum_{i=1}^{N} f\left(x_{0 \ldots t}^{(i)}\right) w\left(x_{0 . \ldots t}^{(i)}\right)
\end{aligned}
$$

A simple example illustrating the concept of importance sampling is in the integration of $g(x)$ in (3.20). Essentially, the integration finds the probability of picking a number $x$ greater than 0 , but less than 1 from the exponential distribution. Performing the integration analytically, the solution is found to be $1-e^{\frac{-1}{100}}=0.00995$. With the analytical solution, it can be seen that if normal Monte Carlo sampling is used to solve this integration, only $1 \%$ of the samples selected would be relevant to the integration, implying a large waste of computation.

With the alternative distribution in (3.21), importance sampling can be used to reduce computation wastage. With the alternate exponential distribution, the probability of picking a number $x$ within the desired range is calculated to be $1-e^{-1}=$ 0.6321 , or $63.21 \%$ of the time. 


$$
\begin{aligned}
& g(x)=\int_{0}^{1} p(x) d x \\
& p(x)=\frac{1}{100} e^{\frac{-x}{100}} \\
& \pi(x)=e^{-x}
\end{aligned}
$$

To use importance sampling to solve the integration, set $f\left(x_{0 \ldots t}\right)=1$, and use (3.19) with i.i.d. samples drawn from $\pi(\cdot)$. The approximation is run with differing numbers of samples, and the results are displayed in Table 3.9. As can be seen, the estimates offer good results even though a completely different distribution is used.

Table 3.9: Summary of Importance Sampling Approximations to Simple Integral

\begin{tabular}{|c|c|c|}
\hline Samples & Analytic Solution & Estimated Solution \\
\hline \hline 10 & \multirow{3}{*}{0.009950166} & 0.009949984 \\
& & 0.009956032 \\
& & 0.009957363 \\
& & 0.009958402 \\
\hline 10,000 & &
\end{tabular}

In this example, the alternate distribution is used to reduce computation requirements, but other aforementioned reasons, such as ease of sampling, are also good reasons for choosing a suitable alternate distribution.

Different forms of importance sampling are possible; another formulation is given in (3.22) as taken from [37]. Given a function $f\left(s_{0 \ldots t}\right)$ to be estimated,

$$
E\left[f\left(s_{0 \ldots t}\right)\right]=\frac{\int f\left(s_{0 \ldots t}\right) w\left(s_{0 \ldots t}\right) \pi\left(s_{0 \ldots t} \mid d_{0 \ldots t}\right) d s_{0 \ldots t}}{\int w\left(s_{0 \ldots t}\right) \pi\left(s_{0 \ldots t} \mid d_{0 \ldots t}\right) d s_{0 \ldots t}}
$$

where

$$
w\left(s_{0 \ldots t}\right)=\frac{p\left(s_{0 \ldots t} \mid d_{0 \ldots t}\right)}{\pi\left(s_{0 \ldots t} \mid d_{0 \ldots t}\right)} .
$$


This formulation is the same as the one shown in (3.18), except it has a denominator portion. The denominator serves as a normalisation factor, as is seen later in (3.23).

Given that i.i.d. samples $s_{0 \ldots t}^{(i)}=\left\{s_{0 \ldots t}^{(1)}, \ldots, s_{0 \ldots t}^{(N)}\right\}$ are again simulated from $\pi(\cdot)$ to give $\Pi_{N}(\cdot)$, then

$$
\begin{aligned}
E\left[f\left(s_{0 \ldots t}\right)\right] & =\frac{\int f\left(s_{0 \ldots t}\right) w\left(s_{0 \ldots t}\right) \Pi_{N}\left(s_{0 \ldots t} \mid d_{0 \ldots t}\right) d s_{0 \ldots t}}{\int w\left(s_{0 \ldots t}\right) \Pi_{N}\left(s_{0 \ldots t} \mid d_{0 \ldots t}\right) d s_{0 \ldots t}} \\
& =\frac{\sum_{i=1}^{N} f\left(s_{0 \ldots t}\right) w\left(s_{0 \ldots t}\right) \delta\left(s-s_{0 \ldots t}^{(i)}\right)}{\int w\left(s_{0 \ldots t}\right) \delta\left(s-s_{0 \ldots t}^{(i)}\right) d s_{0 \ldots t}} \\
& =\frac{\sum_{i=1}^{N} f\left(s_{0 \ldots t}^{(i)}\right) w\left(s_{0 \ldots t}^{(i)}\right)}{\sum_{i=1}^{N} w\left(s_{0 \ldots t}^{(i)}\right)}
\end{aligned}
$$

the expression in (3.23) is exactly the same form as in (3.19) after substituting $\Pi_{N}(\cdot)$. The state $x_{0 \ldots t}$ is analogous to $s_{0 \ldots t}$, while $y_{0 \ldots t}$ is analogous to $d_{0 \ldots t}$. The only difference between these two formulations is their weight functions. The latter formulation has a normalisation factor that is due to the denominator in the approximation, and rescales the weights so that they sum up to one.

The latter formulation is used in [37] for robot localisation. For robot localisation, $s_{0 \ldots t}$ corresponds to the state of the robot at time $t$. The function $f\left(s_{0 \ldots t}\right)$ is $s_{t}$, since the state of the robot is the object of interest, while $d_{0 \ldots t}$ corresponds to actions $a_{0 \ldots t}$ and observations $o_{0 \ldots t}$ of the robot like in (2.2). When importance sampling is used for robot localisation, the alternate distribution is

$$
\pi\left(s_{t} \mid d_{0 \ldots t}\right)=p\left(s_{t} \mid s_{t-1}, a_{t-1}\right) \operatorname{Bel}\left(x_{t-1}\right) .
$$

The actual distribution of interest is

$$
p\left(s_{t} \mid d_{0 \ldots t}\right)=\eta p\left(o_{t} \mid s_{t}^{(i)}\right) p\left(s_{t}^{(i)} \mid s_{t-1}^{(i)}, a_{t-1}\right) \operatorname{Bel}\left(s_{t-1}^{(i)}\right)
$$


Therefore, the weights are calculated by:

$$
\begin{aligned}
w^{(i)} & =\frac{\text { target distribution }}{\text { proposal distribution }} \\
& =\frac{\eta p\left(o_{t} \mid s_{t}^{(i)}\right) p\left(s_{t}^{(i)} \mid a_{t-1} s_{t-1}\right) \operatorname{Bel}\left(s_{t-1}\right)}{p\left(s_{t}^{(i)} \mid a_{t-1} s_{t-1}\right) \operatorname{Bel}\left(s_{t-1}\right)} \\
& =\eta p\left(o_{t} \mid s_{t}^{(i)}\right)
\end{aligned}
$$

Importance sampling, however, is not a satisfactory solution since it does not accomodate a recursive solution. All data $d_{0 \ldots t}$ needs to be available for the procedure, and the complexity of the operation grows without bounds as time goes on. Sequential importance sampling is described in the next section that provides a satisfactory recursive solution.

\subsubsection{Sequential Importance Sampling}

Sequential Importance Sampling (SIS) provides for a satisfactory recursive solution by building on the method of importance sampling. The alternate distribution is limited to the following factorized form

$$
\begin{aligned}
\pi\left(s_{0 \ldots t} \mid d_{0 \ldots t}\right) & =\pi\left(s_{0 \ldots t-1} \mid d_{0 \ldots t-1}\right) \pi\left(s_{n} \mid s_{0 \ldots t-1}, d_{0 \ldots t}\right) \\
& =\pi\left(s_{0}\right) \prod_{k=1}^{n} \pi\left(s_{k} \mid s_{0 \ldots k-1}, d_{0 \ldots k}\right)
\end{aligned}
$$


With this constraint, the weights derived in importance sampling can then be estimated with the following recursive relationship after some manipulation:

$$
\begin{aligned}
w_{t}^{(i)} & \propto \frac{p\left(s_{0 \ldots t}^{(i)} \mid d_{0 \ldots t}\right)}{\pi\left(s_{0 \ldots t}^{(i)} \mid d_{0 \ldots t}\right)} \\
& \propto \frac{p\left(d_{t} \mid s_{0 \ldots t}^{(i)}, d_{0 \ldots t-1}\right) p\left(s_{0 \ldots . t}^{(i)} \mid d_{0 \ldots t-1}\right)}{p\left(d_{t} \mid d_{0 \ldots t-1}\right)}\left(\frac{1}{\pi\left(s_{0 \ldots t}^{(i)} \mid d_{0 \ldots t}\right)}\right) \\
& \propto \frac{p\left(d_{t} \mid s_{t}^{(i)}\right) p\left(s_{t}^{(i)} \mid s_{0 \ldots t-1}^{(i)}\right) p\left(s_{0 \ldots t-1}^{(i)} \mid d_{0 \ldots t-1}\right)}{p\left(d_{t} \mid d_{0 \ldots t-1}\right)}\left(\frac{1}{\pi\left(s_{t}^{(i)} \mid s_{0 \ldots t-1}^{(i)}, d_{0 \ldots t-1}\right) \pi\left(s_{0 \ldots t-1}^{(i)} \mid d_{0 \ldots t-1}\right)}\right) \\
& \propto w_{t-1}^{(i)} \frac{p\left(d_{t} \mid s_{t}^{(i)}\right) p\left(s_{t}^{(i)} \mid s_{0 \ldots t-1}^{(i)}\right)}{p\left(d_{t} \mid d_{0 \ldots t-1}\right) \pi\left(s_{t}^{(i)} \mid s_{0 \ldots t-1}^{(i)}, d_{0 \ldots t-1}\right)}
\end{aligned}
$$

With the recursive weight update relationship, there is no longer any requirement of having all data $d_{0 . . . t}$ to recalculate the weights. The weights may be updated as data are sequentially received. SIS filters belong to the family of particle filters, and the samples that approximate continuous distributions in such filters are also known as particles. There is, however, a degeneracy problem that is associated with SIS filters.

It is known that plain SIS particle filters are affected by degenerate particles whereby most of the particles in the filter have low weights that do not affect the quantity estimated by the particle filter. As indicated in [38], the variance of the importance weights can only increase over time, causing this problem. As such, the majority of computation time is spent on degenerate particles that do not contribute much to the final estimation. There are two possible techniques to avoid this degeneracy: proper selection of the importance function, and resampling. The type of particle filter used in the Monte Carlo localisation process is usually one that uses the resampling technique. This is described in Section 3.2.4. 


\section{Selection of Importance Function}

Through proper selection of the importance function $\pi(\cdot)$, the variance of the importance weights $w(\cdot)$ can be minimized, thus avoiding the issue of degenerate particles. This is to say, the spread of the weight values would not be large, and there would not be many particles that have low weight values that do not contribute to the answer. The ideal importance function would be the true joint density function $p(\cdot)$, then the weight of all particles would be a value of one. However, an alternate importance function might have been chosen for efficiency, or availability reasons, thus the selection of the importance function may not be a tunable parameter. There has been some work and applications of optimal importance functions in $[1,28,66,75,139]$.

\section{Filter Resampling}

In resampling the particles $s_{0 \ldots t}^{(i)}=\left\{s_{0 \ldots t}^{(1)}, \ldots, s_{0 \ldots t}^{(N)}\right\}$, particles with low weights are removed, thus eliminating the degenerate particle problem. There are a variety of resampling techniques such as algorithms based on order statistics [20, 104], stratified sampling [76], systematic resampling [65], among others [19, 48, 49]. These all belong to the family of particle filters.

The most popular of resampling techniques is the Sampling Importance Resampling (SIR) filters introduced by Rubin in [107] and popularised by Gordon et al. in [50] with their weighted bootstrap filter. As described, the standard bootstrap SIR filter continuously iterates through the following steps:

Prediction: particles passed through system model to get the prior

Update: particles evaluated and weights recalculated upon receipt of measurements 
Resampling: resample with replacement from the discrete distribution specified by the filter particles

This algorithm, introduces the problem of sample impoverishment because there is a lack of diversity amongst the resampled particles. The problem is enhanced if the true posterior density lies in a region of low prior density. A possible solution is to use large numbers of particles, thereby approximating the continuous prior density better, and preventing a "collapse" of the approximation to a few highly weighted particles. This, however, is not computationally tractable, and alternate suggestions include introducing jitter to "roughen" particles and spread them out. Most recent resampling techniques previously mentioned take this problem into account. This method of dealing with degenerate particles is typically used in particle filters for Monte Carlo localisation.

\subsubsection{Monte Carlo Localisation}

Monte Carlo localisation (MCL) was first introduced by Burgard, Fox, Dellaert, and Thrun in a series of papers, [127] being a key paper. MCL could be regarded as a definition of the localisation problem for use with particle filters to solve the global localisation, and tracking problem. However, there are two distinct stages to the MCL

filter: initialisation (sampling), and particle filtering (recursive update). Initialisation seeds the particle filter with initial hypotheses for the state of the robot, while particle filtering refines the hypotheses to estimate the true state of the robot. Typically, a weighted bootstrap filter is used for the particle filtering process. 


\section{Initialisation Stage}

In the initialisation stage of the MCL process, the particle filter is seeded with particles sampled from an initial continuous probability density distribution for the state of the robot. Any distribution may be used, but common ones are the uniform and the Gaussian distributions.

The uniform distribution is typically used when no knowledge of the robot's initial state in the environment is known, and it may be anywhere in the known free-space with equal probability (Figure 3.3a). With a grid-based map, however, the uniform distribution can be expressed as in (3.27). Every position in the map is equally likely, however, this does not account for the third dimension of robot state, the orientation angle $\theta$ of the robot at a given position. The angle $\theta$ must also be chosen for a particular location followng (3.28). All possible angles are equally likely.

$$
\begin{aligned}
& p(\operatorname{Cell}(i, j))=\frac{1}{(N * \vartheta)^{2}}, \quad \text { for } \mathrm{N} \text { empty cells } \\
& \qquad \text { is the size of each square cell } \\
& p(\theta)=\frac{1}{2 \pi}
\end{aligned}
$$

The Gaussian distribution is typically used when an initial starting state for the robot is known, with some amount of uncertainty (Figure 3.3b). The implemented MCL filter is seeded with a Gaussian distribution. To approximate the effect of a uniform distribution, one can specify a Gaussian distribution with large variance to spread out the particles sampled from the known freespace. Particles are then sampled from this distribution, so that the particles are i.i.d.. 


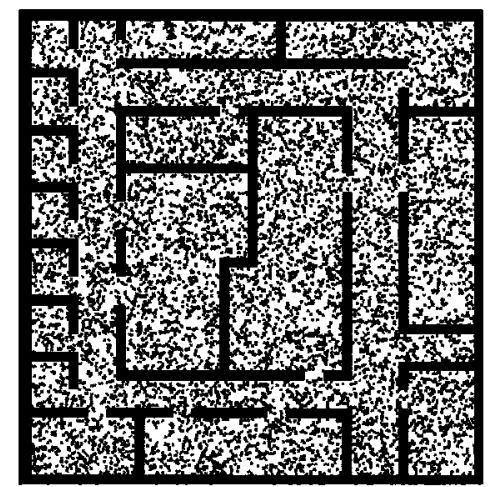

3.3a) Uniform Distribution Seed for Localisation

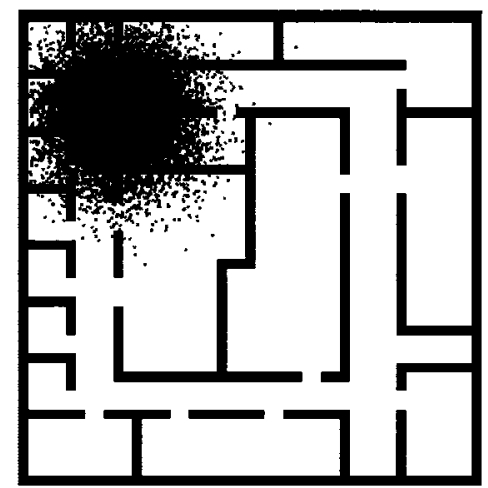

3.3b) Gaussian Distribution Seed for Localisation

Figure 3.3: Initial Seed Distributions for Localisation

\section{Particle Filtering Stage}

The particle filtering stage is essential to arrive at an updated posterior position distribution after integrating the actions and sensor observations of the robot. It is the main component of the MCL method, and depends on the type of particle filter used. Many variant particle filters are available, and the following particle filters are described in the following sections:

- Weighted Bootstrap Filter

- Clustered Particle Filter

- Uniform Particle Filter

- Clustered Uniform Particle Filter

\section{Weighted Bootstrap Filter}

The weighted bootstrap filter $[50,107]$ is the standard particle filter that is used for MCL. The algorithm for the bootstrap filter is shown in Table 3.10, with specific 
reference for use in robot localisation. More details about the operation and implementation of the algorithm is given in Section 4.5.3. The bootstrap filter is an SIR filter that is described in Section 3.2.2.

Table 3.10: Weighted Bootstrap Filter Algorithm for Use in MCL

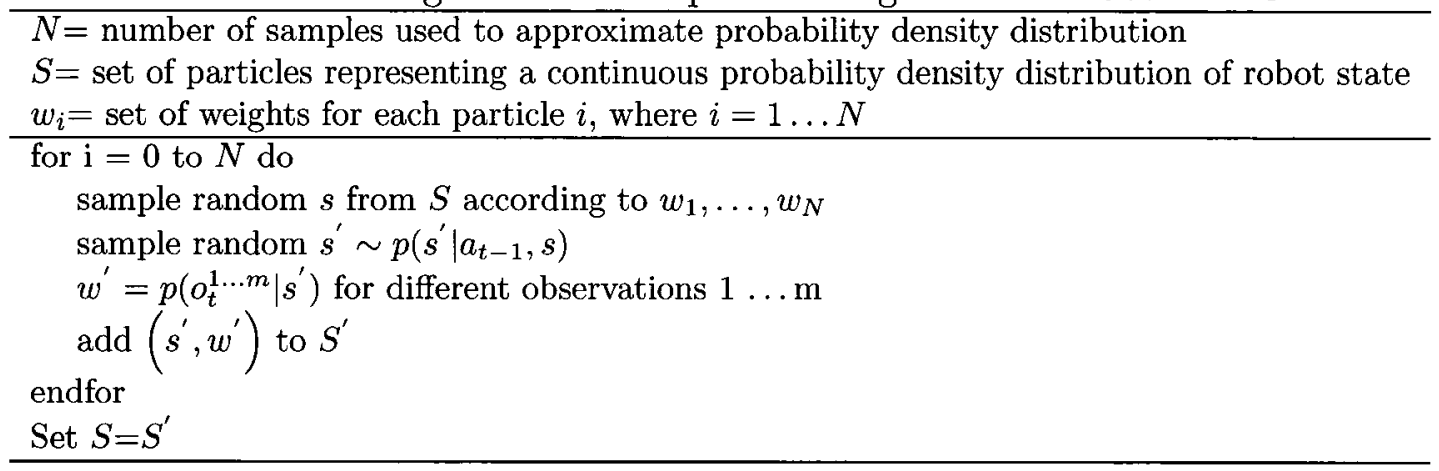

\section{Clustered Particle Filter}

From Section 3.2.2, the importance factor for samples in a particle filter is calculated by (3.26), reproduced in the following for convenience:

$$
\begin{aligned}
w^{(i)} & =\frac{\text { target distribution }}{\text { proposal distribution }} \\
& =\frac{\eta p\left(o_{t} \mid s_{t}^{(i)}\right) p\left(s_{t}^{(i)} \mid a_{t-1} s_{t-1}\right) \operatorname{Bel}\left(s_{t-1}\right)}{p\left(s_{t}^{(i)} \mid a_{t-1} s_{t-1}\right) \operatorname{Bel}\left(s_{t-1}\right)} \\
& =\eta p\left(o_{t} \mid s_{t}^{(i)}\right)
\end{aligned}
$$

In [86], Milstein, Sánchez, and Williamson take a slightly modified view of the normal particle filtering process. Milstein et al. included the use of $f_{t}\left(s_{t}\right)$, a positive function over the state space, in their calculations for a more general weight update equation. Given that the particle filter is already properly initialised using a seed distribution, then $S_{t}$ is the set of samples selected from the seed distribution. There are two steps to refine the estimation of the robot's state: 
1. $N$ random particles $s_{t-1}=\left\{s_{t-1}^{(1)} \ldots s_{t-1}^{(N)}\right\}$ are selected from $S_{t-1}$. These samples are distributed according to $f_{t-1}\left(s_{t-1}\right) p\left(s_{t-1} \mid d_{0 \ldots t}\right)$.

2. Update the particles $s_{t-1}$ to become $s_{t} \sim p\left(s_{t} \mid a_{t}, s_{t-1}\right)$.

The modified weight update equation is then:

$$
\begin{aligned}
w^{(i)} & =\frac{\text { target distribution }}{\text { proposal distribution }} \\
& =\frac{f_{t}\left(s_{t}^{(i)}\right) p\left(s_{t}^{(i)} \mid d_{0 \ldots t}\right)}{f_{t-1}\left(s_{t-1}^{(i)}\right) p\left(s_{t}^{(i)} \mid a_{t}, s_{t-1}\right) p\left(s_{t-1} \mid d_{0 \ldots t-1}\right)} \\
& =\frac{f_{t}\left(s_{t}^{(i)}\right) \eta p\left(o_{t} \mid s_{t}^{(i)}\right) p\left(s_{t} \mid a_{t-1} s_{t-1}\right) B e l\left(s_{t-1}\right)}{f_{t-1}\left(s_{t-1}^{(i)}\right) p\left(s_{t}^{(i)} \mid a_{t}, s_{t-1}\right) p\left(s_{t-1} \mid d_{0 \ldots t-1}\right)} \\
& =\eta p\left(o_{t} \mid s_{t}^{(i)}\right) \frac{f_{t}\left(s_{t}^{(i)}\right)}{f_{t-1}\left(s_{t-1}^{(i)}\right)}
\end{aligned}
$$

As can be seen, this result is similar to the normal weight equation, but includes the positive function $f\left(s_{t}\right)$ that normally would be included in the normalisation factor $\eta$. With this analysis, Milstein et al. asserts that modified proposal distributions may be used.

In designing the function $f\left(s_{t}\right)$ for use in the modified proposal distribution, a number of assumptions are made:

- there are $K$ clusters

- every particle is assigned to a single cluster. Let $c\left(s_{t}^{(i)}\right)$ be a function that returns the cluster number that a particle $s_{t}^{(i)}$ belongs to

- $f\left(s_{t}\right)$ gives the same value for each particle that is in the same cluster

- the cumulative weight over all particles in a cluster is equal to the cumulative 
weight in all other clusters:

$$
\sum_{\substack{(i) \in s_{t}^{(i)} \\ s_{t} \\ c\left(s_{t}^{(i)}\right)=k}} f\left(s_{t}^{(i)}\right) p\left(s_{t}^{(i)} \mid d_{0 . \ldots t}\right)=\sum_{\substack{(i) \\ s_{t}^{(i)} \in S_{t} \\ c\left(s_{t}^{(i)}\right)=k^{\prime}}} f\left(s_{t}^{(i)}\right) p\left(s_{t}^{(i)} \mid d_{0 . . t}\right)
$$

- a particle, once assigned to a cluster, cannot change to another cluster

A function that satisfies these criteria is $f\left(s_{t}^{(i)}\right)=\frac{1}{B_{k, t}} \cdot B_{k, t}$ is the probability that a cluster $k$ contains the actual robot state at time $t$.

$$
\begin{aligned}
B_{k, t} & =p\left(k_{t} \mid d_{0 . \ldots t}\right) \\
& =\eta \int p\left(o_{t} \mid k_{t}\right) p\left(k_{t} \mid a_{t} k_{t-1}\right) p\left(k_{t-1} \mid d_{0 \ldots t-1}\right) d k_{t-1}
\end{aligned}
$$

The form of $B_{k, t}$ shown in (3.32) is similar in form to $\operatorname{Bel}\left(s_{t}\right)$, the probability distribution that $s_{t}$ is the actual robot state as derived in Section 2.3. Due to the use of a finite number of samples to approximate the distribution, $B_{k, t}$ is actually

$$
B_{k, t}=\frac{\eta}{m_{k}} \sum_{k=1 \ldots K} p\left(o_{t} \mid k_{t}\right) p\left(k_{t} \mid a_{t} s_{t-1}\right) p\left(k_{t-1} \mid d_{0 \ldots t}\right),
$$

for $m_{k}$ samples in cluster $k$.

The assumption that particles cannot change clusters after assignment gives rise to the following:

$$
\begin{array}{rlrl}
p\left(k_{t} \mid a_{t} k_{t-1}\right) & =0, & & k_{t} \neq k_{t-1} \\
& =1, & k_{t}=k_{t-1} .
\end{array}
$$

With (3.34), it can be seen from (3.33) that $B_{k, t} \propto p\left(o_{t} \mid k_{t}\right) p\left(k_{t-1} \mid d_{0 \ldots t-1}\right)$. Since a cluster $k$ is composed of samples $s_{t}^{(i)}, p\left(o_{t} \mid k_{t}\right)$ is necessarily related to $p\left(o_{t} \mid s_{t}\right)$ with the relation

$$
p\left(o_{t} \mid k_{t}\right) \propto p\left(k_{t-1} \mid d_{0 \ldots t-1}\right) \sum_{\substack{\left.s_{t}^{(i)} \in S_{t} \\ \text { cos } \\ c s_{t}^{(i)}\right)=k}} p\left(o_{t} \mid s_{t}^{(i)}\right)
$$


Using (3.35) and (3.32),

$$
\begin{aligned}
b_{k, t} & \propto p\left(o_{t} \mid k_{t}\right) p\left(k_{t-1} \mid d_{0 . \ldots t}\right) \\
& \propto p\left(k_{t-1} \mid d_{0 \ldots t-1}\right) \sum_{\substack{s_{t}^{(i)} \in S_{t} \\
c\left(s_{t}^{(i)}\right)=k}} p\left(o_{t} \mid s_{t}^{(i)}\right) \\
& \propto B_{k, t-1}^{2} \sum_{\substack{s_{t}^{(i)} \in S_{t} \\
c\left(s_{t}^{(i)}\right)=k}} p\left(o_{t} \mid s_{t}^{(i)}\right)
\end{aligned}
$$

Therefore, the function $f\left(s_{t}\right)=\frac{1}{B_{k, t}}$ satisfies all the assumptions originally defined, and also has a recursive relationship to $B_{k, t-1}$. The idea of clustering particles is thus mathematically proven from the perspective of importance sampling.

As shown, the modified proposal distribution, namely, the inclusion of $f\left(s_{t}\right)$ changes the particle filter so that weights for samples in the filter are clustered, and weights modified so that each cluster's cumulative weight is equal to all other clusters. That is to say, each cluster is equally likely from the perspective of the particle filter. So, even if a cluster is unlikely to contain the true robot state, the particle filter will not remove the cluster. The distribution $B_{k, t}$ must be looked at to determine the likelihood of the actual robot state belonging in any of the clusters $k=1 \ldots K$ instead. The cluster with the most likely $B_{k, t}$ is the most likely cluster, while clusters with unlikely $B_{k, t}$ must be removed.

Rather than maintain a single particle filter with a complicated weight update procedure, it is equally valid to maintain a series of standard weighted bootstrap particle filters, one for each cluster. This is valid due to the aforementioned assumptions. Table 3.11 gives an implementation of a clustered particle filter using a series of weighted bootstrap filters, as suggested by Milstein et al. . 
Table 3.11: Algorithm for a Clustered Particle Filter

Initialisation

1. seed a bootstrap particle filter with initial seed distribution

2. iterate several steps through the particle filter

3. cluster particles based on spatial similarity

4. create a weighted bootstrap particle filter for each cluster

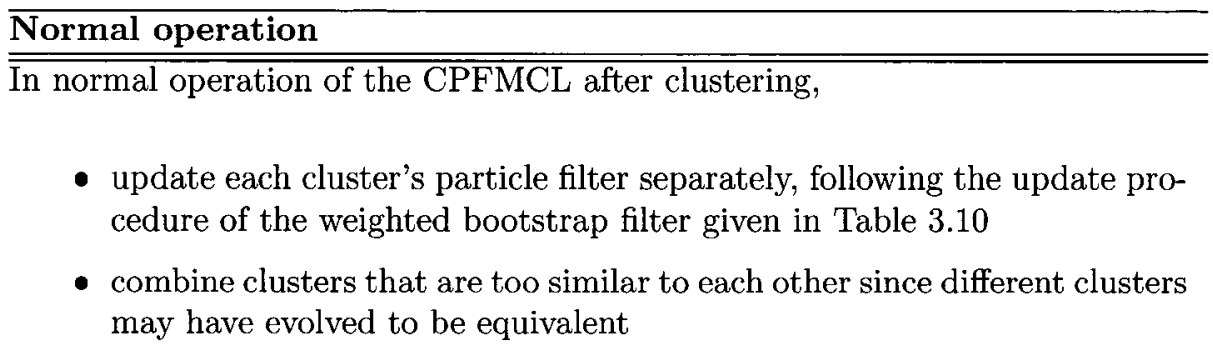

\begin{tabular}{l} 
Additional \\
\hline A separate particle filter is run occasionally in the background to globally \\
relocalise the robot. This allows the method to find likely clusters of particles \\
that may not have been chosen by the initial seeding process due to the use of \\
insufficient particles, or use of an inappropriate seeding process. It also allows \\
for the solution of the kidnapped robot problem.
\end{tabular}

\section{Uniform Particle Filter}

In the normal procedure for MCL, the sensor model, $p\left(o_{t} \mid s_{t}\right)$, and the action model, $p\left(s_{t} \mid a_{t} s_{t-1}\right)$, need to be defined. These distributions are typically assumed Gaussian due to its simplicity and widespread applicability due to theorems like the Central Limit Theorem. For example, the use of a Gaussian distribution for the sensor model of a range sensor may seem reasonable. If a distance $r$ is the ideal range to be sensed by the range sensor, the reported range $\hat{r}$ may be distributed according to a Gaussian due to noise. Due to the unpredictability of the environment, however, objects might get in the way of the range sensor, and the resulting range reported by the range sensor will resemble that of a uniform distribution. Ueda et al. make 
this argument in [130] to use a uniform distribution for the sensor and action model. The application of a uniform distribution to the action model is justifiable since the robot described in [130] is a quadruped robot. In such a robot, the motion is highly variable. Repeated motion of the robot under the same input control results in different travelled distances. Including the possibility of unsensed collisions, a uniform distribution is more accurate as the motion model for the quadruped robot. If the expected motion is a distance $d$, the actual distance travelled follows a uniform distribution defined by $f(x)=\frac{1}{d}$; having not moved at all is just as likely as having moved a distance $d$.

Since this variant MCL process is dependent on uniform distributions, the variant particle filter is called Uniform MCL (U-MCL). The algorithm is shown in Table 3.12. Ueda et al. implemented U-MCL for their robot dog that was entered into the Robocup competition, and compared the U-MCL localisation results to Sensor Resetting Localisation (SRL) [70], another MCL variant. Their results indicated that U-MCL performed as well as SRL did. More details on the implementation of this algorithm is given in Section 4.5.3.

\section{Uniform Clustered Particle Filter}

Combining the ideas of both CPF-MCL, and U-MCL, a new variant particle filter named Uniform Clustered Particle Filter MCL (UCPF-MCL) is proposed in this section. As developed in Section 3.2.4, clustering particles into separate individual particle filters is mathematically plausible. Each cluster of particles has a separate weighted bootstrap filter that evolves the particles of the cluster. With UCPF-MCL, U-MCL filters are used to evolve each cluster of particles, rather than a weighted bootstrap filter like in CPF-MCL. The algorithm is shown in Table 3.13, and is very 
Table 3.12: Algorithm for a Uniform MCL Particle Filter

Algorithm follows the standard weighted bootstrap filter algorithm in Table 3.10 with the following changes

Sensor update

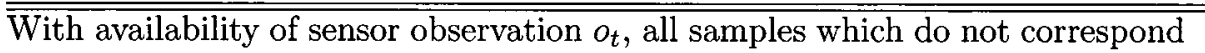
to $p\left(o_{t} \mid s_{t}\right)$ are removed from the filter. ie: robot states for which the observation is unlikely are removed. The sensor model $p\left(o_{t} \mid s_{t}\right)$ is based on a uniform distribution.

Action update

If $d$ is the expected distance travelled, then each sample $s_{t-1}^{(i)}$ is updated with a distance $d^{\prime}$. The quantity $d^{\prime}$ is selected from a uniform distribution specified by $f(x)=\frac{1}{d}$.

Resampling

If the number of samples $n$ in the U-MCL filter is less than the max number of particles $N$ allowed in the U-MCL filter, then new samples are added through the resampling process. Two samples are selected at random, and a new sample is added at the midpoint of the two samples. Repeat the process until $n=N$.

similar to the CPF-MCL algorithm.

This clustering extension to the normal U-MCL is necessary due to the normal U-MCL resampling process. The normal resampling process selects two particles at random, and a new particle is created at the midpoint of the two particles. This resampling strategy is not expected to give good results in a general environment, even though it gave good results as published in [130]. In [130], the robot is placed in a Robocup soccer field environment. Its sensors are able to determine its approximate location on the soccer field, and results only in a unimodal belief distribution. In a general environment, multimodal distributions are possible to describe the state of the robot due to environmental symmetry.

By clustering, each cluster in UCPF-MCL is evolved using a U-MCL filter that is unimodal, and therefore the resampling process works as expected. Without clustering, a different resampling process is needed, as suggested in [130]. 
Table 3.13: Algorithm for a Uniform Clustered Particle Filter Initialisation

1. seed a U-MCL particle filter with initial seed distribution

2. iterate a single step through the U-MCL filter

3. cluster particles based on spatial similarity

4. create a U-MCL particle filter for each cluster

\section{Normal operation \\ In normal operation of the UCPF-MCL after clustering, \\ - update each cluster's U-MCL particle filter separately \\ - combine clusters that are too similar to each other since different clusters may have evolved to be equivalent}

\section{Additional}

Relocalise the filter if all clusters are considered improbable, and eliminated. First attempt to locally resample from the most likely cluster to attempt and recover from losing track of the robot location. If the local resampling fails, then the filter is re-initialised with the uniform distribution.

\subsection{Chapter Summary}

Detailed theoretical examinations of various mapping and localisation methods are given in this chapter.

Grid based mapping methods are examined in this chapter. Grid based maps consists of arrays of cells. Each cell is mappable to an area in the real environment, and numerical values are associated with each cell to give an occupancy confidence level. These occupancy values need to be updated according to sensor observations over time as the robot moves through the environment. These sensor observations are prone to error, and are potentially contradictory. Two methods are considered for use in such a merge: the Bayesian method, and the evidential method. The evidential method, based on Dempster-Shafer evidential theory, is shown to be more robust and 
powerful in that it is able to represent the unknown, and reason about uncertainty. Furthermore, it is mathematically equivalent to the Bayesian method under certain constraints, therefore, evidential theory is more general than Bayesian theory, and encompasses it. The evidential grid based mapping method is implemented in the simulator described in Chapter 4, and examined using simulated results in Chapter 5.

The theory behind MCL methods is given in this chapter, starting with a fundamental look at ideal Monte Carlo sampling. MCL is essentially a process to solve the robot localisation problem using particle filters. Specific particle filters described in this chapter are the weighted bootstrap filter, the clustered particle filter, the uniform particle filter, and the newly proposed uniform clustered particle filter. These localisation methods are implemented in the simulator of Chapter 4, and their simulated results examined in Chapter 5. 


\section{Chapter 4}

\section{Simulator Implementation}

Mapping deals with the problem of creating a map of the environment given noisy sensor observations, and ideal motion. Mapping assumes that the localisation problem is solved prior to the mapping sequence. Localisation deals with the problem of locating a robot's location in an environment, given that a map of the environment is available beforehand. Therefore, localisation assumes that the mapping problem is solved prior to the localisation process. To experiment with the mapping and localisation techniques developed in Chapter 3, a software ulator is designed and created. The simulator is created with the following goals:

- ease of simulation creation, and control

- ease of virtual world creation

- ease of simulation capability enhancement

This chapter describes the implementation details of the simulator. 


\subsection{Component Overview}

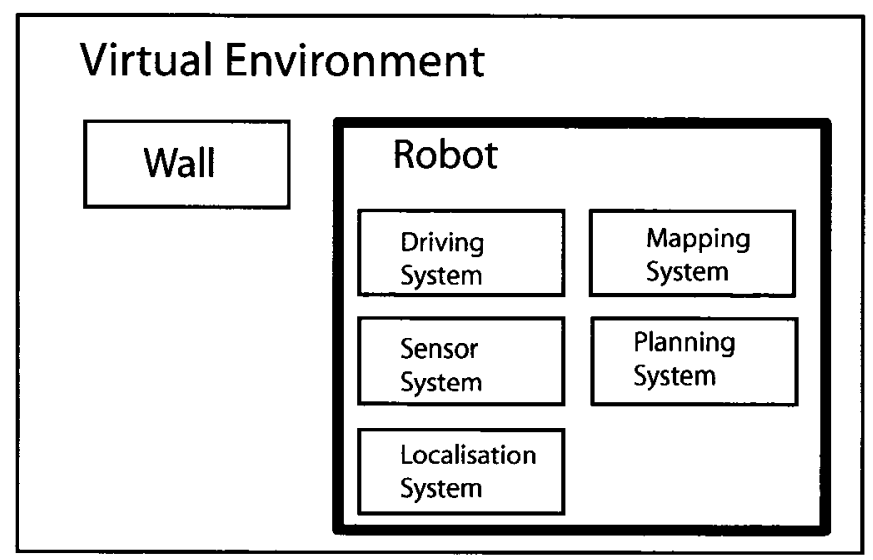

Figure 4.1: Simulator Component Diagram

The simulator is composed of three main interesting components: simulator components, mapping components, and localisation components. Figure 4.1 illustrates the components that constitutes the simulator as a whole.

The simulator components simulate a minimal number of details about the real world required to experiment with different mapping and localisation techniques. There are only two types of objects in the world, the walls, and the robots. Many robots may be placed within the environment, and the robot itself is composed of many different components. Other details of the real world are considered extraneous for this simulation, such as floors, and doors. The addition of flooring, for example, allows simulation of scenarios where the robot moves through different terrain conditions. This is described in Section 4.3.

Other auxiliary components exist as part of the simulator that deal with the graphical user interface and configuration purposes, but are not discussed in detail 
here. Extra details about the simulator are available in Appendix A.

Mapping components deal directly with the mapping process. The grid based evidential mapping method described in Section 3.1 is implemented here. The components of interest are the mapping system itself, and the sensor model that converts simulated sensor observations into a form usable by the mapping system. These are described in Section 4.4.

Localisation components deal directly with the localisation process. The standard Monte Carlo localisation (MCL) technique is implemented, along with the variants, namely, Clustered Particle Filtering MCL (CPF-MCL), Uniform MCL (U-MCL), and Uniform Clustered Particle Filtering MCL (UCPF-MCL). The components of interest are the localisation systems themselves, and sensor and action models that convert observations and actions of the robot into a form usable by the localisation systems. These are described in Section 4.5.

\subsection{Implementation Tools}

Various major technologies were used in the implementation of the simulator:

Java3D: Java3D is the platform for simulator implementation to take advantage of Java3D's rendering features for conveying the state of the virtual world to the user. Other similar technologies such as OpenGL were considered, but Java3D easily provided the required capabilities.

JFC/Swing: Java Foundation Classes/Swing is the graphical user interface toolkit used to create the user interface.

JNI: Java Native Interface is the Java technology used to allow mixed language 
development. Using JNI allows interfacing $\mathrm{C} / \mathrm{C}++$ to the Java program.

$\mathrm{C} / \mathrm{C}++: \mathrm{C} / \mathrm{C}++$ provides advantages such as speed, and use of existing libraries of code without the use of which would otherwise require re-implementation from scratch in Java.

GSL: The GNU Scientific Library (GSL) is a $\mathrm{C} / \mathrm{C}++$ library that provides functionality commonly required in scientific $\mathrm{C} / \mathrm{C}++$ programs. The scope of the library is immense, but some of the functionality used in the simulator include random number generation, random number distributions, matrix manipulation, vector manipulation, and matrix algebra.

JavaCC: JavaCC is known as the Java Compiler Compiler, a parser and lexical analyser generator. JavaCC takes meta-language describing language grammar, and generates Java code that is used to parse a file using that language construct. JavaCC is used to parse the simulator configuration file, as well as $\log$ file processing to generate statistics.

\subsection{General Component Implementation}

This section describes the general framework and implementation of components that simulate the virtual environment for the robot. The components of Figure 4.1 are discussed in this section.

\subsubsection{Wall}

The wall is simply a rectangular block in the virtual environment that obstructs a robot's movement. Multiple walls may be created and combined to form a more 
complex environment. Once created in the virtual environment, walls may not be destroyed or modified online.

\subsubsection{Robot}

The Robot is the general class of components which model virtual robots that may move around in the virtual world. A Robot is represented by an avatar in the virtual world, and controls various aspects of itself such as its heading direction, or its speed. It is also composed of four helper subsystems: the driving system, the sensor system, the localization method, and the planning system. Embedding different subsystems in the Robot gives the robot with different capabilities, since each of these subsystems control various properties and behaviour of the robot in the virtual world.

\section{SimpleRobot}

The SimpleRobot is a specific implementation that belongs to the Robot class of components. Asthetically, the robot is represented by a cylinder shaped avatar. Technically, the SimpleRobot models a robot with two drive wheels that can be independently controlled for a wide range of motion, such as the robot depicted in Figure 3.1. Since there are two wheels independently driven to achieve motion, the robot maintains the wheel speeds for each of the drive wheels to simulate a differential drive system. Rather than maintaining the actual wheel rotation speeds, however, SimpleRobot maintains the ideal ground speed of each point on the robot where a drive wheel is mounted. Since the rotational speed of a motor cannot instantaneously change in real life, neither can the speeds of the robot change instantaneously. As such, the individual wheel speeds are modeled as a state space discrete linear system with specifiable order and parameters. The linear system used is a second order system. 
Other qualities of the robot are controlled by its subsystems.

\subsubsection{Driving System}

The driving system is a general class of components that is responsible for simulating realistic movement dynamics of the robot in the virtual world. It is well known that although a real robot may command a certain amount of movement, many external factors such as wheel slip, collisions, and motor skippage will affect the actual movement. In many cases, it will cause the movement to be less than expected, or in some cases, be completely different from expected. This system emulates such details of the real world to control the movement of the robot in the virtual environment such that it remains realistic.

\section{SimpleDrive}

The SimpleDrive is an implementation of a driving system that may be embedded within a Robot. It injects noise based on a differential drive system into its actual simulated motion. In a real environment, there are many sources of noise that can enter into the actions of the robot. For example, though the robot may execute its wheel motion exactly to move a certain distance, it may not be able to achieve that distance due to wheel slippage or collisions with external objects. Noise in a differential drive system will arise from wheel slip from either of the drive wheels, thus causing the robot to displace itself less than expected. If the wheel slippage from the drive wheels is unequal, the robot will also undergo unexpected rotation. SimpleDrive injects Gaussian noise of specifiable variance to the expected ideal speed of the wheels so that the actual ground speed of the robot where the drive wheels are mounted are corrupted with noise. 


\subsubsection{Sensor System}

The sensor system is a collection of various sensors that may be mounted on the robot.

Sensors are the only method for the robot to gather information about its environment. There are two simulated sensors: the odometry sensor, and the laser range sensor.

\section{Odometry Sensor}

Dead reckoning allows a robot to guess its position relative to its starting location based on previous motion. Thus, keeping track of odometry is essential for dead

reckoning. Equipment such as wheel encoders may be necessary for odometry calculations, but the odometry sensor is an abstraction of all the equipment necessary to calculate odometry.

The implementation of the odometry sensor projects the distance travelled based on the commanded speeds of the robot. This ideal distance may not correspond with the actual distance travelled due to the noise injected by the driving system.

\section{Laser Range Sensor}

Laser range sensors are used to measure the distance to the closest object in the direction that the laser is aimed.

There are many types of lasers available that work to sense range using different operating principles such as triangulation, time-of-flight, interferometry, or pattern projection [8]. Pattern projection, for example, works well to model a $3-\mathrm{D}$ representation of an object with distinctive textures, but does not present similar advantages when scanning flat textureless walls. 
It is assumed that the robot is to work in an indoor environment. Indoor environments are characterised by vertically indistinct structures. It is due to the assumption that indoor environments are vertically indistinct that the map is collapseable into a 2-D occupancy grid model, without need to encode vertical information.

Point or scanning lasers would be most useful to map the environment. Working based on triangulation or time-of-flight, these lasers provide sparse range information restricted to the direction they are sensing. Therefore, a good model of a point laser is a finite length ray. Assuming the state of the robot within the map is known, the finite length ray can be traced within the map. This is illustrated in Figure 4.2. Though real laser range sensors typically make use of triangulation techniques with sample averaging, the software model does not include such intricacies. The laser sensor software model returns the distance to the closest object in the direction in which it is aimed through use of ray tracing.

The distance returned from the simulator is the true distance. As with practical sensors, the measured distance is typically noisy. There are many factors which contribute to the noisiness, such as multi-path reflections, texture of object, angle of incidence, etc. Therefore, the measured range may be expressed as

$$
\hat{r}=r+\nu,
$$

where $\hat{r}$ is the measured range, $r$ is the true range, and $\nu$ is a noise process. Due to the many noise factors, $\nu$ is assumed to be Gaussian distributed. Also, a valid method of obtaining a range measurement is the averaging of many laser range samples; another justification for the Gaussian distributed noise due to the Central Limit Theorem. For a noisy sensor, the $\nu$ process will have a large variance, whereas an accurate sensor will have a small variance for the $\nu$ process. Therefore the software laser sensor 
model corrupts the true range with a Gaussian noise process to give a simulated noisy measured range.

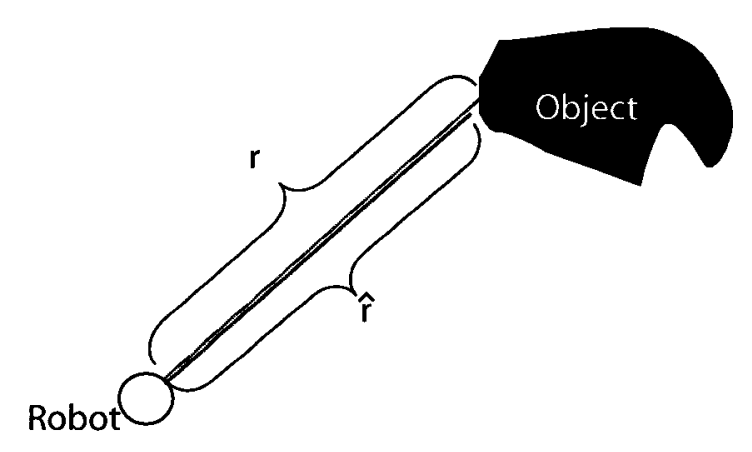

Figure 4.2: Laser Range Measurement Scenario

\subsubsection{Mapping System}

The mapping system allows the robot to map its environment based on sensor observations. The specific implementation is described in Section 4.4.

\subsubsection{Localisation System}

The localisation system performs data fusion upon gathered sensory input so as to estimate the robot's pose within the virtual world. This embeds within the robot a certain amount of intelligence about its location so that it may plan its future actions. Implemented localisation systems are described in Section 4.5.

\subsubsection{Planning System}

The planning system provides the robot with the capability to create and follow trajectories through the environment so that manual control is not needed. 


\section{SimplePathPlanner}

The SimplePathPlanner is an implementation of the planning system that gives the robot the capability to execute predetermined absolute paths through the environment. The paths are provided by the user and is akin to manually controlling the robot through use of remote control.

\subsection{Mapping Component Implementation}

This section discusses components specific to mapping capability for the robot in the virtual environment.

\subsubsection{Laser Sensor}

The software laser sensor model described in Section 4.3.4 simulates $\hat{r}$, noisy range measurements typical of the real world. This noisy range measurement needs to be translated to a form usable by the evidential mapping unit of the robot. Since the mapping unit creates a grid based map, the range information is decomposed as seen in Figure 4.3. It is assumed that the robot's position in the map is known, so that the path of the laser can be ray traced through the map. The cell at the end of the ray is updated with occupied evidence support, while all other cells that intersect with the ray's path are updated using empty evidence support. The basic probability 
assignment (BPA) that gives occupied evidence support is

$$
\begin{aligned}
m_{i, j}(O) & =\lambda_{1} \\
m_{i, j}(E) & =0 \\
m_{i, j}(O E) & =1-\lambda_{1} \\
m_{i, j}(\phi) & =0 .
\end{aligned}
$$

The BPA that gives empty evidence support is

$$
\begin{aligned}
m_{i, j}(O) & =0 \\
m_{i, j}(E) & =\lambda_{2} \\
m_{i, j}(O E) & =1-\lambda_{2} \\
m_{i, j}(\phi) & =0 .
\end{aligned}
$$

$\lambda_{1}$ represents the numerical degree of belief that the laser sensor believes the cell to be occupied. Likewise, $\lambda_{2}$ represents the belief for a cell to be empty. For an ideal laser sensor, the values are chosen to be $\left(\lambda_{1}, \lambda_{2}\right)=(1.0,1.0)$. For a noisy sensor, the values should not be such extreme values, since that will create contradictory beliefs, and the state of the cell will not converge to a stable value. Though the distance $\hat{r}$ is a noisy measurement, it is expected that the evidential mapping method will merge the noisy measurements so as to create a good grid map of the environment. $\lambda_{1}, \lambda_{2}$ are adjustable parameters of the laser range sensor model that are specified for test cases in Section 5.2 to observe the effect of varying these parameters.

\subsubsection{EvidentialMapping}

The EvidentialMapping component is an implementation of the mapping system that can be added to a robot. It provides mapping capabilities to the robot, and is based 


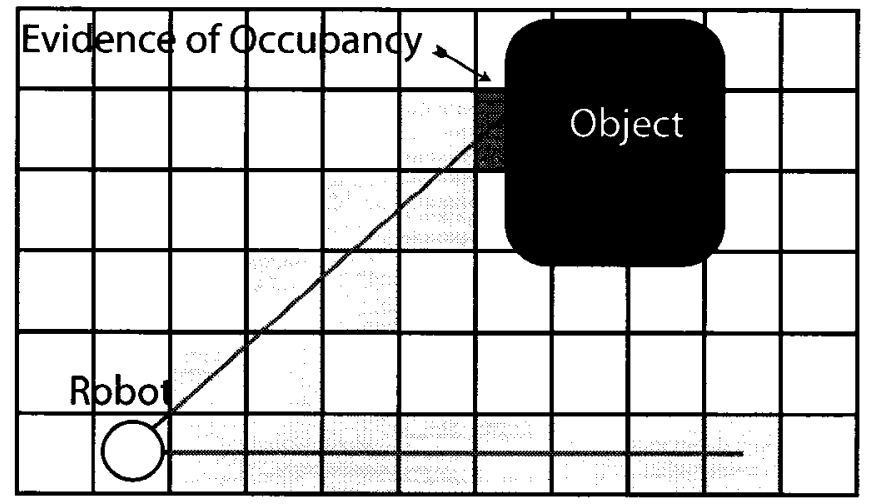

Shaded rectangles are provided with evidence of emptiness

Figure 4.3: Illustration of Laser Sensor Model for Mapping

on a grid map discussed in Section 3.1. The grid based map uses Dempster-Shafer theory to integrate independent sources of sensor observations into the map, and is called evidential mapping.

Briefly, the evidential mapping module divides the environment into arrays of small cells. Each cell models the state of the environment at a particular location, and is based on Dempster-Shafer theory. The model used is developed in Section 3.1.5, and due to the structure of the model, two values need to be kept for each cell: the amount of occupancy support, and the amount of emptiness support for each cell. The two support values are updated according to new observations that are integrated into the map. It is not necessary to keep the amount of unassigned support since it can be calculated from the two values that are kept due to the small scale of the problem.

Given that the evidential mapping method considers solely the mapping problem, the mapping method integrates new sensor observations into the map with the assumption that the robot's location is known. The laser sensor model for evidential mapping described in Section 4.4.1 provides occupancy or emptiness support for cells 
in the grid map, and these observations are integrated using conjunctive integration. There are concrete examples provided in Section 3.1.6 that give detailed information on the procedure of evidential mapping.

\subsection{Localisation Component Implementation}

This section gives details on components specific to the localisation process for the robot in the simulated environment.

\subsubsection{Odometry Sensor}

The odometry sensor component described in Section 4.3.4 simulates an odometry sensor in the virtual environment. The odometry sensor returns the projected ideal distance travelled by the robot according to commanded motion. This ideal distance does not coincide with the actual distance travelled due to motion noise. An action and sensor model is defined so that this estimated distance can be used for localisation purposes, as mentioned in Section 2.3 and Section 3.2.4.

The action model is $p\left(s_{t} \mid a_{t-1} s_{t-1}\right)$. That is a continuous distribution, but since the MCL method deals with sample based particle filters, a sample based action model is desired. For each particle $s_{t-1}^{(i)}$, a new particle $s_{t}^{(i)}$ is proposed based on the action taken in the previous time step. The new particles are distributed according to the distribution $p\left(s_{t} \mid s_{t-1} a_{t-1}\right) \operatorname{Bel}\left(s_{t-1}\right)$.

Suppose $\Delta \hat{d}=(\delta x, \delta y, \delta \theta)$ is the ideal estimated distance travelled, as reported by the odometry sensor's measurements. It is expected that the actual distance travelled is given by $\Delta d=\left(\delta x+\nu_{1}, \delta y+\nu_{2}, \delta \theta+\nu_{3}\right)$, where $\nu$ are Gaussian noise processes. The Gaussian noise models the effect of inaccurate motion due to practical actuators. 
The action model has to update the particles in the particle filter with the motion estimate. Since the actual distance travelled is expected to be a noisy version of the ideal distance reported by the odometry sensor, instances of odometry readings are sampled from $\Delta d$ for each particle, and the particle updated with that odometry reading.

This is illustrated with a 1-D example: suppose the initial state of the robot that can only move in one dimension is known exactly to be $s_{0}=\left(x_{0}\right)$. The particle filter is initialised with $N$ particles $s_{0}^{(i)}=s_{0}, \forall i=1 \ldots N$. Given that the robot moves an actual distance of $\Delta d$, and that the odometry sensor of the robot registers an odometry of $\Delta \hat{d}$, the particles in the filter need to be updated in such a way that the filter correctly proposes a particle that is the true state $s_{1}$. Each particle is updated with a different instance of $\Delta d$, distributed according to the noise distribution $\nu$. This scenario is illustrated in Figure 4.4. As the number of particles $N \rightarrow \infty$, the continuous distribution $\Delta d$ is represented exactly after the particle update, and the true state $s_{1}$ is definitely included within it. The irrelevant particles will be eliminated with subsequent sensor observations.

A sensor model is also defined for the odometry sensor. After proposing new particles based on odometry, the proposed particles are checked. The sensor model is $p\left(o_{t} \mid s_{t}\right)$, the probability of having observation $o$ with given states $s$. Once again, due to the sample based particle filter, a sample based sensor model is implemented. Given the particles $s_{t}^{(i)}$, each particle is checked for the possiblity of the action. If a particle ends up being in occupied space according to the known map, then it is assigned zero probability, while a particle that remains in free-space is assigned a high probability. 


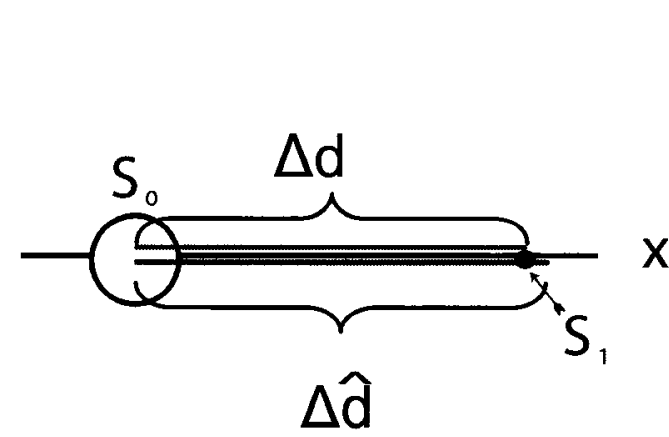

4.4a) Actual odometry measurement scenario

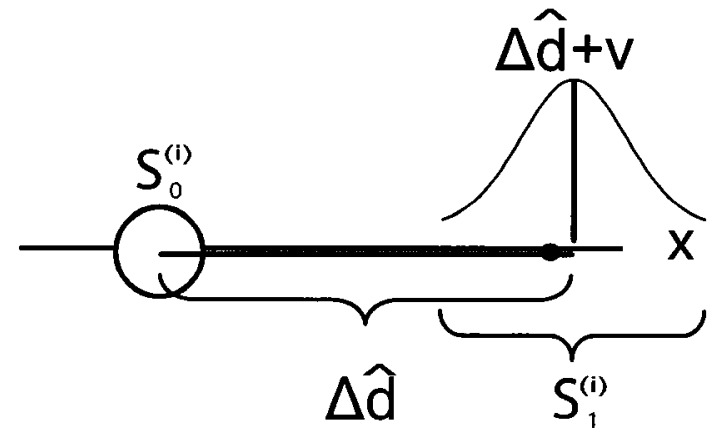

4.4b) Action model particle propagation

Figure 4.4: Odometry Measurement Scenario for Localisation

\subsubsection{Laser Sensor}

The software laser sensor model described in Section 4.3 .4 simulates $\hat{r}$, noisy range measurements typical of the real world. This noisy range measurement needs to be translated to a form usable by the localisation component of the robot. Two such laser sensor models exist in the simulator used by different localisation methods. One is based on the Gaussian distribution, and the other is based on the uniform distribution. This sensor model is mentioned in Section 2.3, and is the distribution associated with $p\left(o_{t} \mid s_{t}\right)$, the probability of an observation given a state distribution. There is no action model associated with the laser range sensor.

Since particle filters represent continuous distributions with samples, a sample based sensor model is implemented. Given a state $s_{t}^{(i)}$, and the noisy range measurement $\hat{r}, p\left(o_{t} \mid s_{t}^{(i)}\right)$ is given.

\section{Gaussian Model}

For a candidate robot state $s_{t}^{(i)}$, the robot position can be pinpointed on the map $m$. Based on the robot position, ranges are determined by ray tracing the finite length 
laser ray according to the map to get $\tilde{r}$, the expected range if $s_{t}^{(i)}$ is the actual robot state. A possible scenario is illustrated in Figure 4.5.

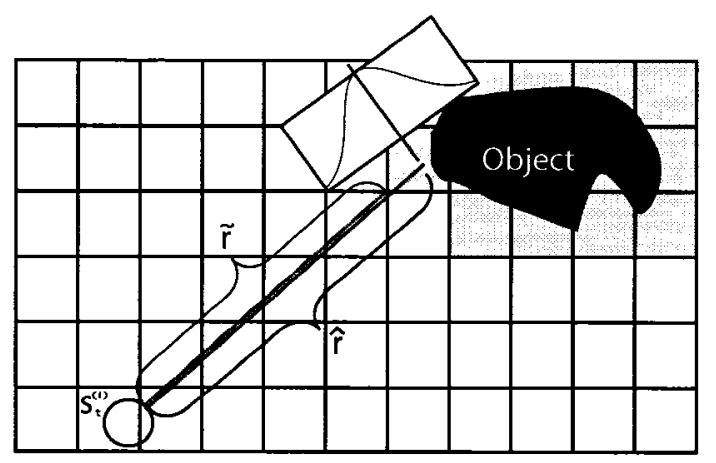

4.5a) Possible candidate state given high weight

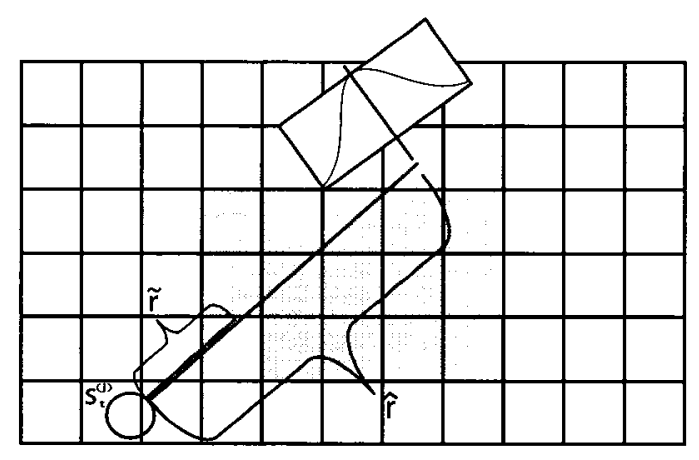

4.5b) Possible candidate state given low weight

Figure 4.5: Laser Range Measurement Scenario for Localisation

The probability of measuring $\hat{r}$ given that the true value is to be $\tilde{r}$ is given by their difference shown in (4.3). The probability from the sensor model is used in the weighting process for resampling in the weighted bootstrap filter described in Section 4.5.3.

$$
\begin{aligned}
& \Delta r=\hat{r}-\tilde{r} \\
& p\left(o_{t} \mid s_{t}\right)=\frac{1}{\sqrt{2 \pi \sigma^{2}}} e^{-\frac{\Delta r^{2}}{2 \sigma^{2}}}
\end{aligned}
$$

\section{Uniform Model}

The uniform model to the sensor model $p\left(o_{t} \mid s_{t}\right)$ is implemented in a similar fashion to the Gaussian model. This laser model is illustrated in Figure 4.6. If the robot's position is well represented by a given particle, then the robot should observe a distance $\tilde{r}$ from a given mounted laser range sensor. The distance $\tilde{r}$ is calculated through comparison with the environment map given to the localisation method prior to placing the robot in the environment. If the measured distance $\hat{r}$ of that given 
laser range sensor is within a distance $\epsilon$ of the expected distance $\tilde{r}$, then it has the same probability of occuring as compared to any other distance within an $\epsilon$ distance. Therefore, the sensor model is

$$
p\left(o_{t} \mid s_{t}^{(i)}\right)=\left\{\begin{array}{lc}
\frac{1}{2 \epsilon} & |\tilde{r}-\hat{r}|<\epsilon \\
0 & \text { otherwise }
\end{array}\right.
$$

Since the same uniform model is used for all particles, if observations lie within $\epsilon$ of $\tilde{r}$, then the weight of all particles that remain probable according to this model is assigned to be $\frac{1}{2 \epsilon}$. The weights become $\frac{1}{N}$ upon normalisation, where $N$ is the total number of particles in the filter. The uniform laser range sensor model is, in essence, removing all particles whose range measurements $\hat{r}$ do not lie within an $\epsilon$ of the expected range measurement $\tilde{r}$. In this thesis, $\epsilon$ is set to 0.5 .

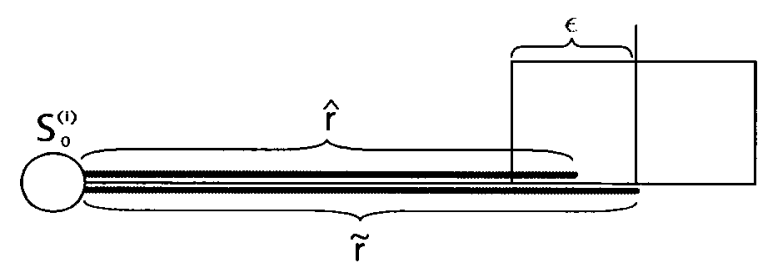

Figure 4.6: Uniform Laser Range Sensor Model for Localisation

\subsubsection{Monte Carlo Localisation}

The Monte Carlo localisation (MCL) system is an implementation of a localisation method. MCL is detailed in Section 3.2.4, and is composed of two stages: initialisation (sampling), and particle filtering (recursive update). 


\section{Initialisation}

In the initialisation stage of the MCL filter, the filter needs to be seeded with particles sampled from an initial continuous probability density distribution for the pose of the robot. Any distribution may be used, but common ones are the Gaussian, or the uniform distribution. The initialisation portion of the MCL process is implemented as described in Section 3.2.4.

\section{Particle Filtering}

The particle filter used in the standard MCL process is the weighted bootstrap filter, and is implemented following the algorithm shown in Table 3.10.

\section{Example}

This section illustrates a concrete example of the MCL localisation method using a weighted bootstrap filter. In the standard MCL process, the robot is given a static grid map of the environment that it is placed within. Upon start up, the robot does not have any indication of its location, thus it must perform global localisation. The bootstrap filter is thus initialised using particles sampled from a 3-D uniform distribution over the free space of the environment using (3.27) and (3.28) as described in Section 3.2.4. Each particle is also assigned equal weight since they are all equally probable. This is illustrated by Figure 3.3a, a screen shot of the initialisation process taken from the simulator.

After the initialisation stage, the recursive update stage begins. For each localisation attempt, the weighted bootstrap algorithm detailed in Table 3.10 is executed. The update may be regarded as a series of steps to integrate new action and sensor 
information into the particle filter, and more detail about each step is given in the following:

1. Sample a random particle $s$ from $S$, the set of total particles that represents the weighted hypotheses of robot state. This is a discrete distribution, and more heavily weighted particles are more probable robot states, thus more likely to be selected and updated in the process. The selected particle belongs to the distribution $\operatorname{Bel}\left(s_{t-1}\right)$.

2. Sample a random $s^{\prime}$ given the selected particle $s$, and previous action $a_{t-1}$ executed by the robot. $s^{\prime}$ is a predicted particle that may exist in the posterior position distribution of the robot, and depends on the action model of the actions that the robot executed (ie: odometry sensor's action model). This particle belongs to the proposal distribution for $p\left(s_{t} \mid s_{t-1} a_{t-1}\right) \operatorname{Bel}\left(s_{t-1}\right)$ that is part of the prediction phase of the recursive Bayesian update equation shown in $(2.6 \mathrm{~d})$.

3. Generate $w^{\prime}$, the probability of $s^{\prime}$ being the pose of the robot conditioned on the observations of the sensors. This probability depends on the sensor model of the sensors mounted on the robot, such as the laser range sensor Section 4.5.2, or the odometry sensor Section 4.5.1. This probability is used as the importance weight associated with the sampled particle so that we can use the proposal distribution to arrive at the desired distribution $\eta p\left(o_{t} \mid s_{t}^{(i)}\right) p\left(s_{t}^{(i)} \mid s_{t-1}^{(i)} a_{t-1}\right) \operatorname{Bel}\left(s_{t-1}\right)$ following the ideas of sequential importance sampling, and samples importance re-sampling given in Section 3.2.3.

4. Repeat steps 1 to 3 until enough particles are sampled, according to the number of particles to be kept by the filter. 
5. Normalise weights for all the updated particles so that they form a discrete probability distribution, the desired distribution.

After this process is executed, only initial particles drawn from the uniform distribution that approximate the robot's location well according to sensor and action data remains. These steps are executed for every localisation attempt made by the robot. It should be noted that particle filters make use of a large number of particles to approximate the continuous distributions that exist in the recursive Bayesian update equation in (2.6d), and implements that equation directly by using the particle approximations.

\subsubsection{Clustered Particle Filtering MCL}

The CPF-MCL is a variant of the MCL process, and like MCL, is composed of two stages: initialisation, and particle filtering. The algorithm given in Table 3.11 details both stages, and the implementation closely follows that algorithm. Since the CPF-MCL algorithm closely parallels the standard bootstrap filter used in MCL, no example execution of the CPF-MCL algorithm is given. While the CPF-MCL filter is in the initialisation stage, it should be regarded as the regular MCL process with additional steps to eventually cluster the initial particles. Once the CPF-MCL filter is in the particle filtering stage, it should be regarded as having multiple MCL processes running together. Each MCL process is responsible for updating clusters of particles at different spatial locations. 


\section{Initialisation}

The robot's initial location is unknown, so the particle filter is seeded with particles sampled from a 3-D uniform distribution spread over the whole environment. The particle filter used to evolve the initial seed particles is the standard weighted bootstrap filter, therefore, the CPF-MCL method is currently equivalent to the standard MCL process described in Section 4.5.3.

According to $[85,86]$, the CPF-MCL algorithm specifies that the initial particles are to be updated using the bootstrap filter for several iterations before clustering the remaining particles into groups according to their spatial proximity to each other in all three dimensions. It is important to execute the bootstrap filter for several iterations to ensure that the particles approximate a belief distribution that has distinct modes. This indicates that the filter has decided upon likely locations of the robot that cannot be eliminated using the current observations of the environment. If the particles are not clustered at this point, valid modes of the belief distribution may eventually be removed from the bootstrap filter. This effect is related to the idea of sample impoverishment described in Section 3.2.3, and the validity of clustering is shown mathematically in Section 3.2.4. The actual number of iterations depends on the environment [86], but experiments indicate that the particles settle to a belief distribution with distinct modes even after the first localisation iteration. Thus, the CPF-MCL method implemented for the experiments in this thesis cluster after only a single iteration of the bootstrap filter.

The clustering algorithm follows [85], but any type of clustering algorithm that clusters on basis of particle proximity should work in-principle. A survey of clustering algorithms is available in [61]. 


\section{Particle Filtering}

After the particles in the initialisation process are clustered, each cluster of particles is updated using their own individual bootstrap filter. Each bootstrap filter continues to follow the process described in Section 4.5.3.

At this stage, the CPF-MCL algorithm also indicates that global localisation should occasionally take place in the background to search for potential clusters of particles which represent the robot's location. If the potential clusters found do not already exist in the CPF-MCL filter, then a bootstrap filter should be created for the cluster and inserted into the CPF-MCL filter. This separate global localisation process should take place periodically after several localisation attempts to conserve computational power. The simulator allows the user to specify the number of localisation attempts before starting a global localisation process, but in the experiments of the thesis, the global localisation process is constantly running in the background.

\subsubsection{Uniform MCL}

The U-MCL is implemented as a variant of the MCL process. It uses a uniform distribution for its sensor and action models with slight changes to the particle filtering portion of the process. It is implemented following the algorithm in Table 3.12. The initialisation stage and the particle filtering stage are detailed in this section.

\section{Initialisation}

U-MCL is initialised following the same procedure as the MCL process described in Section 4.5.3. After this is complete, U-MCL enters the particle filtering stage. 


\section{Particle Filtering}

The particle filtering stage for U-MCL is again similar to the MCL process. The algorithm at this stage is given as a series of steps in the following for clarity:

1. For each particle in the filter, update the particle to account for the action of the robot by predicting new particles based on odometry. The updated particles belong to the distribution $p\left(s_{t} \mid s_{t-1} a_{t-1}\right) \operatorname{Bel}\left(s_{t-1}\right)$ that is the prediction portion of the recursive Bayesian update equation given in (2.6d).

2. For each particle in the filter, use the uniform sensor model described in Section 4.5.2 to decide unlikely particles to be removed from the filter. The remaining particles are distributed according to (2.6d).

3. If the number of remaining particles is less than desired, new particles are sampled from the existing particles. Two particles are chosen at random, and a new particle injected into the filter that occurs at the midpoint of the chosen particles. This resampling process attempts to inject new particles that improves the approximation of the belief distribution around the estimated location of the robot. This resampling process, however, does not work for multimodal belief distributions. Particles from radically different spatial locations may be chosen, and the new particle proposed will be at an incorrect location.

\subsubsection{Uniform Clustered Particle Filtering MCL}

The UCPF-MCL is a variant of both the MCL and the CPF-MCL method. It is very similar to the algorithm of CPF-MCL with two major differences. The UCPF-MCL only uses a bootstrap filter at initialisation, and during global relocalisation. In the 
normal particle filtering stage, for example, the UCPF-MCL spawns a U-MCL filter for each cluster instead. The other major difference is that UCPF-MCL does not have a global localisation task occasionally running in the background to propose new clusters. The UCPF-MCL is implemented following the algorithm Table 3.12, which is very closely related to the algorithm of CPF-MCL given in Table 3.11. Since UCPF-MCL is closely related to CPF-MCL, a concrete example for UCPF-MCL is not given for brevity.

\subsection{Chapter Summary}

This chapter describes the implementation of the simulator developed to simulate various mapping and localisation methods. The general architecture is given, along with the softwares used to create the simulator. Further details are given on how the simulator simulates realistic sensor and motion for the robot. Extra details about sensor and action models for various sensors with respects to mapping, and localisation are also given. This simulator is used to generate the results examined in Chapter 5 . 


\section{Chapter 5}

\section{Simulation Results}

Theoretical fundamentals of various mapping and localisation techniques are outlined in Chapter 3. Using the software simulator developed in Chapter 4, the mapping and localisation techniques are simulated, and the results are presented in this chapter.

\subsection{Simulation Settings}

There are a multitude of settings for the simulator to simulate different scenarios. Some settings that remain constant for all results acquired are the robot configuration, and the test environments that the virtual robot is placed in.

\subsubsection{Robot Configuration}

A realistic robot configuration is used in all simulations, and is based on the robot shown in Figure 3.1. Laser range sensors, stepper motors, grabber mechanisms, and bump sensors are shown on the robot. The items of interest, however, are the laser range sensors, which provide range observations, and the stepper motors, which is a 
key part of the differential drive system. A modified version of the robot's blueprint is shown in Figure 5.1, illustrating the range sensing capabilities of the robot.

In the simulations, some settings for the various subsystems are available. Most of the settings are set through the simulator's configuration file, which is described in more detail in Appendix A. Various settings are described in the following:

Laser Range Sensors: the laser range sensors are assumed to be accurate up to $10 \mathrm{~m}$. Therefore, any distance reading that is further than $10 \mathrm{~m}$ is truncated. The range readings are corrupted with zero mean Gaussian noise of specifiable standard deviation, which varies according to test case settings.

Odometry Sensor: this sensor implements a differential drive noise model. Since the robot is a differential drive robot, it calculates the ideal distance travelled by each of the drive wheels according to the current wheel speeds. These ideal distances are corruptd with a zero mean Gaussian noise whose standard deviation is specified as a percentage of the ideal distance travelled. This noise parameter is specified in the configuration file, and varies according to test case settings.

Mapping Unit: in test cases dealing with evidential mapping, a grid map is kept and updated by the evidential mapping unit. Maps created in this thesis vary in size depending on the size of the environment, but the size of the grid cell is $0.1 \mathrm{~m}$ unless otherwise specified.

Planning Unit: the only path planning system is the SimplePathPlanner that allows the robot to follow absolute paths through the environment. More complex planning units are possible, but are not the focus of this thesis. 


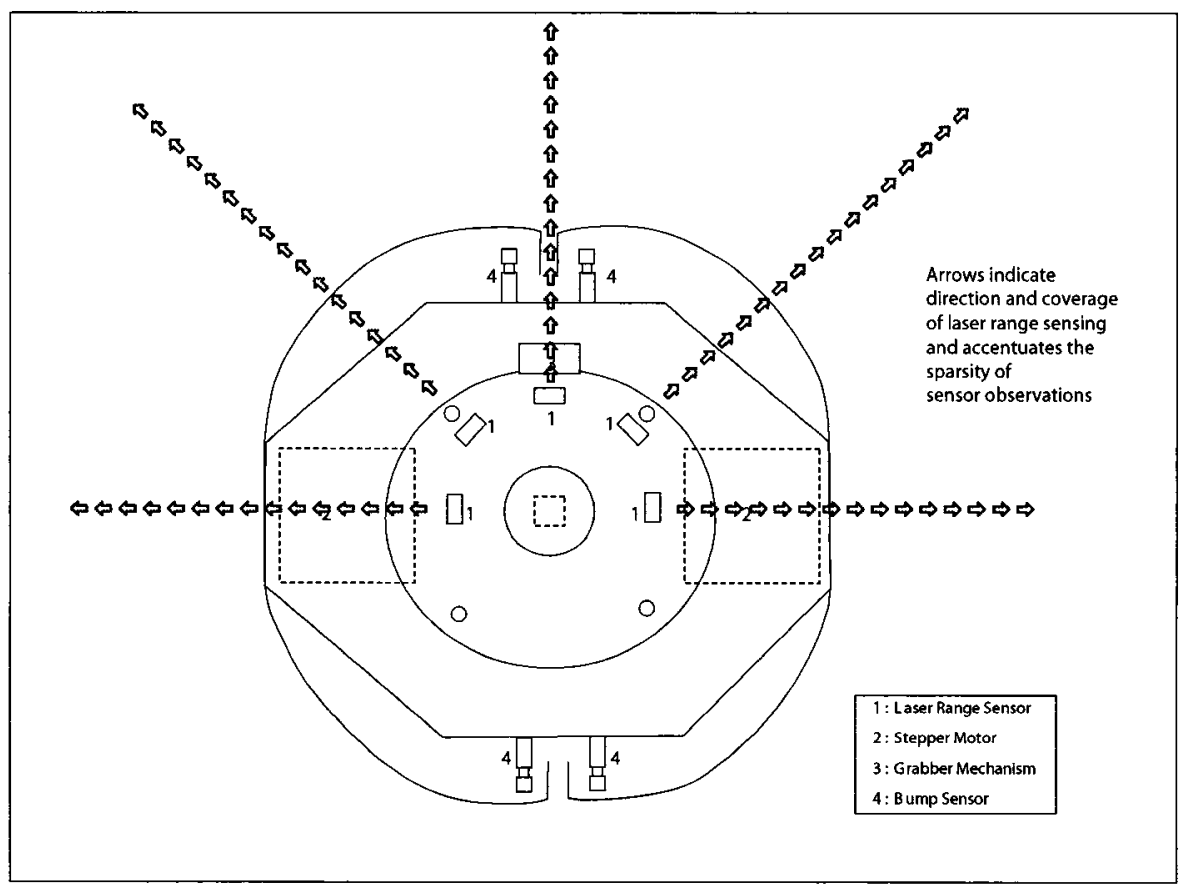

Figure 5.1: Blueprint of Simulated Robot

\subsubsection{Test Environments}

There are two test environments that are used for the remainder of the experiments in this section. The first environment is a simple, small scale symmetric environment whose size is $17 \mathrm{~m}^{2}$. The environment, and the corresponding path that the robot takes through the environment are shown in Figure 5.2a. The symmetric environment is composed of four corridors labeled as left, right, top, and bottom corridors. It should be noted that the top and bottom corridors are longer than the left and right corridors. The second environment is a larger scale office-like environment whose size is $51 \mathrm{~m}^{2}$. Shown in Figure 5.2b is the environment with the corresponding path the robot takes through the environment. It should be noted that the environment has many similar areas, such as the square partitions on the left hand edge of the environment. 


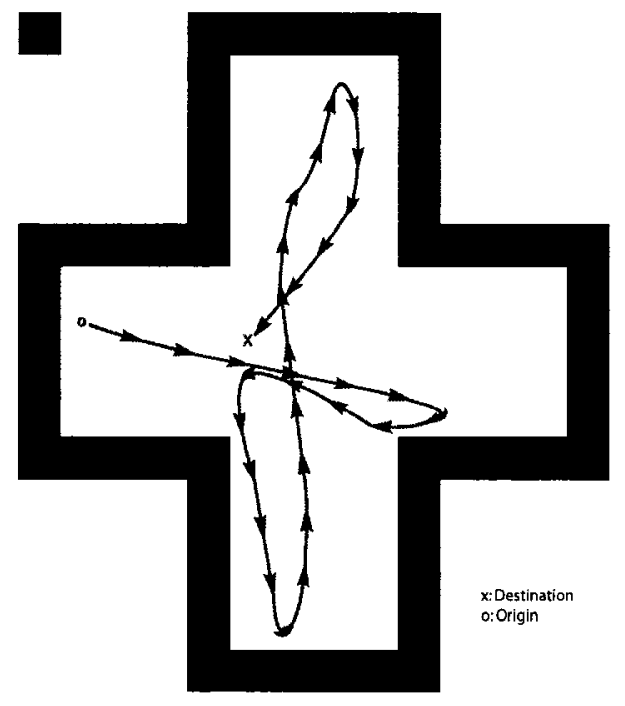

5.2a) Symmetric Environment

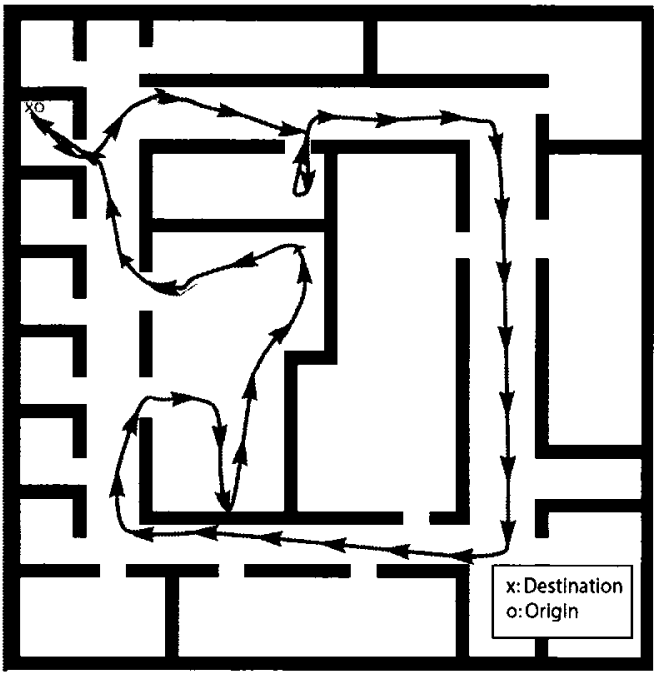

5.2b) Office-like Environment

Figure 5.2: Test Environments

\section{$5.2 \quad$ Mapping}

The evidential mapping method is shown in Section 3.1 to be more flexible and general than the Bayesian mapping method, particularly in its mechanisms for representing the unknown. The evidential mapping method is implemented in the simulator, and tested using different parameter settings. The following results are of interest:

- Sensitivity to evidence values provided by the laser sensor

- Sensitivity to noise in range readings given by the laser sensor

- Sensitivity to noise in motion of the robot

\subsubsection{Symmetric Map Test Cases}

Following the laser range sensor model for mapping described in Section 4.4.1, varying amounts of support can be given to cells the sensor thinks is occupied, or empty. The 
value of support for occupancy $\left(\lambda_{1}\right)$ and for emptiness $\left(\lambda_{2}\right)$, as well as the amount of sensor noise is varied to create different test cases. The sensor noise parameter specifies the standard deviation of the Gaussian noise process used in the laser's measurement model. The test cases are run using the symmetric map test environment described in Section 5.1.2. These test cases are listed in Table 5.1, as well as the references to corresponding maps created. In the resulting occupancy maps, black cells indicate occupied cells, while white cells indicate the lack of any support for occupancy in those cells. In the resulting emptiness maps, black cells indicate empty cells, while white cells indicate the absence of any support for emptiness in those cells.

Table 5.1: Mapping Test Cases for Varying Parameters of Laser Sensor

\begin{tabular}{|c|c|c|c|c|}
\hline Test Case \# & $\overline{\overline{\lambda_{1}}}$ & $\overline{\overline{\lambda_{2}}}$ & "Sensor noise & $\overline{\overline{\text { Results }}}$ \\
\hline $1-1$ & \multirow{4}{*}{0.1} & \multirow{4}{*}{0.1} & $0.5 \mathrm{~m}$ & Figure 5.4 \\
\hline $1-2$ & & & $0.3 \mathrm{~m}$ & Figure 5.5 \\
\hline $1-\overline{3}$ & & & $0.1 \mathrm{~m}$ & Figure 5.6 \\
\hline $1-4$ & & & 0 & \\
\hline $2-1$ & \multirow{4}{*}{0.3} & \multirow{4}{*}{0.1} & $0.5 \mathrm{~m}$ & Figure 5.7 \\
\hline$\overline{2-2}$ & & & $0.3 \mathrm{~m}$ & Figure 5.8 \\
\hline$\overline{2-3}$ & & & $0.1 \mathrm{~m}$ & Figure 5.9 \\
\hline $2-4$ & & & $\overline{0}$ & \\
\hline $3-1$ & \multirow{4}{*}{0.1} & \multirow{4}{*}{0.3} & $0.5 \mathrm{~m}$ & Figure 5.10 \\
\hline$\overline{3-2}$ & & & $0.3 \mathrm{~m}$ & Figure 5.11 \\
\hline$\overline{3-3}$ & & & $0.1 \mathrm{~m}$ & Figure 5.12 \\
\hline $3-4$ & & & 0 & \\
\hline $4-1$ & \multirow{4}{*}{0.8} & \multirow{4}{*}{0.1} & $0.5 \mathrm{~m}$ & Figure 5.13 \\
\hline $4-2$ & & & $0.3 \mathrm{~m}$ & Figure 5.14 \\
\hline $4-3$ & & & $0.1 \mathrm{~m}$ & Figure 5.15 \\
\hline $4-4$ & & & $\overline{0}$ & \\
\hline $5-1$ & \multirow{4}{*}{0.1} & \multirow{4}{*}{0.8} & $0.5 \mathrm{~m}$ & Figure 5.16 \\
\hline $5-2$ & & & $0.3 \mathrm{~m}$ & Figure 5.17 \\
\hline $5-3$ & & & $0.1 \mathrm{~m}$ & Figure 5.18 \\
\hline $5-4$ & & & 0 & \\
\hline $6-1$ & \multirow{4}{*}{0.99} & \multirow{4}{*}{0.99} & $0.5 \mathrm{~m}$ & Figure 5.19 \\
\hline $6-2$ & & & $0.3 \mathrm{~m}$ & Figure 5.20 \\
\hline $6-3$ & & & $0.1 \mathrm{~m}$ & Figure 5.21 \\
\hline $6-4$ & & & 0 & \\
\hline 7 & 1 & 1 & * & \\
\hline
\end{tabular}


The results are discussed in the following categories:

- Cases with no sensor noise

- High support values for both occupancy and emptiness

- Low support values for both occupancy and emptiness

- Moderate occupancy support and low emptiness support

- Low occupancy support and high emptiness support

- High occupancy support and low emptiness support

- Low occupancy support and high emptiness support

- Absolute support values for both occupancy and emptiness

\section{Cases with No Sensor Noise}

Test cases 1-4, 2-4, 3-4, 4-4, 5-4, and 6-4 all correspond to cases with zero sensor noise. Such cases are expected to yield the same mapping result, the ideal map, since there is neither motion noise nor sensor noise in the system. The actual results do not yield the ideal map, and have occasional single cell errors near the boundaries. This effect is seen in the occupancy plausibility map of Figure 5.3a. Free cells immediately adjacent to occupied cells are sometimes mistakenly given occupancy support. This is expected to be an artifact of the simulator implementation, and is related to the accuracy of the Java3D technology used. This is verified using separate test cases using higher resolution grid maps (Figure 5.3b). Due to the higher map resolution, the artifacts at the boundaries of the created map are not seen. That map, however, illustrates the effect of having sparse sensor data in a high resolution map. The white 
cells relatively far away from the wall boundaries indicate that the sensors do not provide any observations for those cells. The environment needs to be scanned more carefully to ensure all cells are classified as being empty.

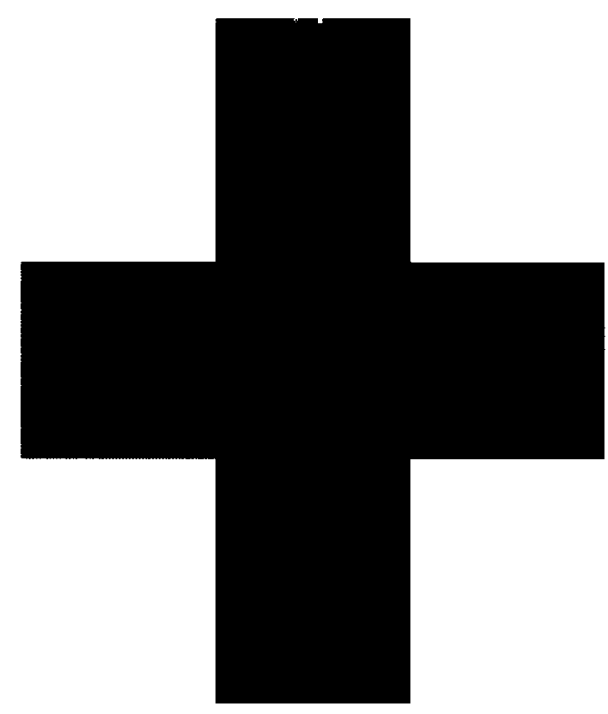

5.3a) Map Resolution of $0.1 \mathrm{~m}$

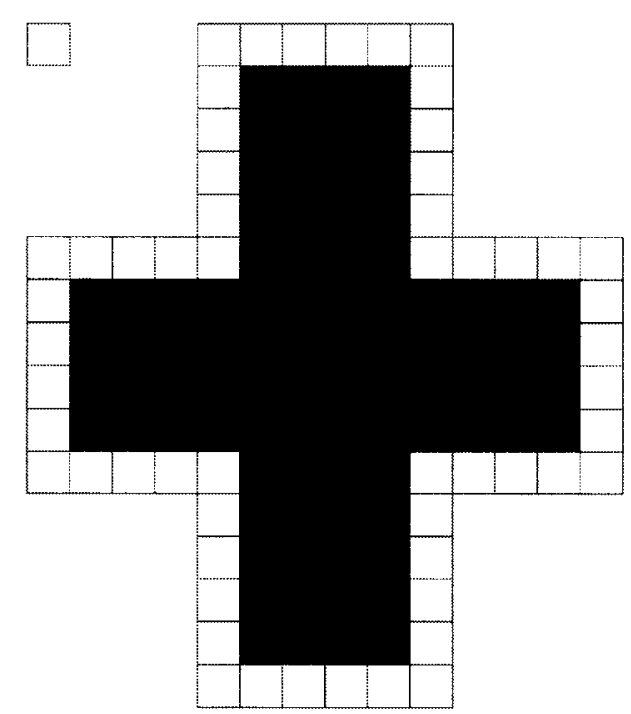

5.3b) Map Resolution of $0.02 \mathrm{~m}$

Figure 5.3: Emptiness Plausibility Maps Created With No Sensor Noise

\section{Low Support for Occupancy and Emptiness}

Test cases 1-1, 1-2, and 1-3 correspond to cases with low occupancy and emptiness support for varying amounts of sensor noise. For test case 1-1, the low support given by the sensor observations for the cells results in larger expanses of grey areas in the support maps, most distinctively shown in Figure 5.4b. The capability of DempsterShafer theory to reason about uncertainty is illustrated when the emptiness support map is contrasted to the emptiness plausibility map (Figure 5.4d). Even though there is not enough support in the support map to be certain of emptiness, the plausibility map illustrates the areas of emptiness correctly. 
From the point of view of Dempster-Shafer theory, portions of the total belief are assigned to emptiness while other portions of the belief are assigned to occupancy. Where there is contradictory belief, the plausibility is decreased, resulting in grey uncertain areas in the plausibility map. From a linguistic point of view, a piece of evidence is accepted completely if there is no conflicting evidence.

Due to the low amount of support for both occupancy and emptiness, the support maps are light shades of grey except where there are many observations that support each other, as in certain areas of the emptiness support map. The dark areas of the emptiness support map illustrates the phenomenon that empty areas of the map tend to get more support due to the sensor model employed; for every cell at the end of the laser ray that gets occupancy support, a lot more cells that intersect the laser ray get emptiness support. Also, comparing the two support maps, neither of them dominates the other to create a "crisp" map. The occupancy support map "bleeds" into the empty space, and vice versa. As a result, the areas around the true boundary between occupancy and emptiness are grey in the plausibility maps indicating uncertainty.

The other test cases have the same effect, but are less noticeable due to the decreased amount of noise.

\section{Moderate Occupancy Support and Low Emptiness Support}

Test cases 2-1, 2-2, and 2-3 correspond to cases with moderate occupancy and low emptiness support for varying amounts of sensor noise. With these parameters, the support for occupancy bleeds into empty areas near the boundary, although it is not as noticeable as the converse test cases 3-1, 3-2, and 3-3 described in the next section. 


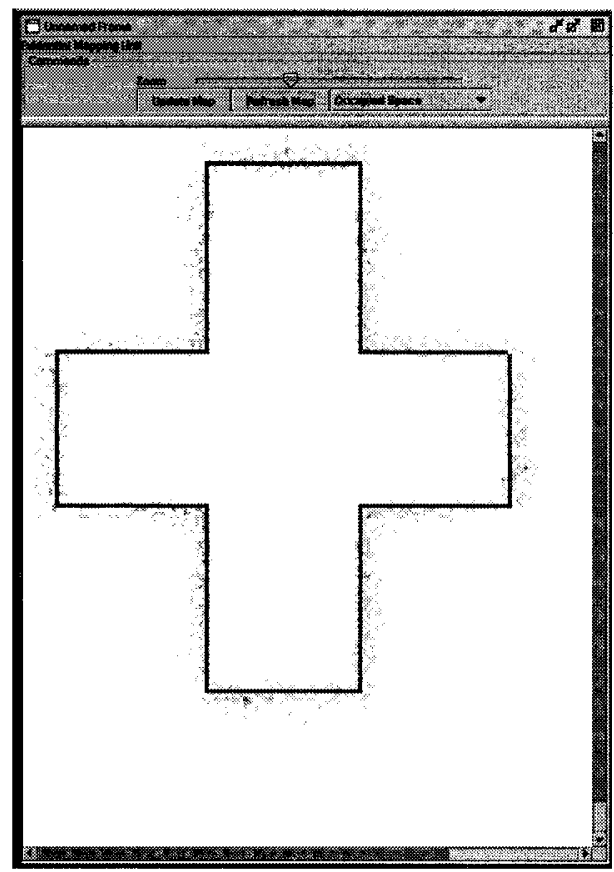

5.4a) Occupancy Support

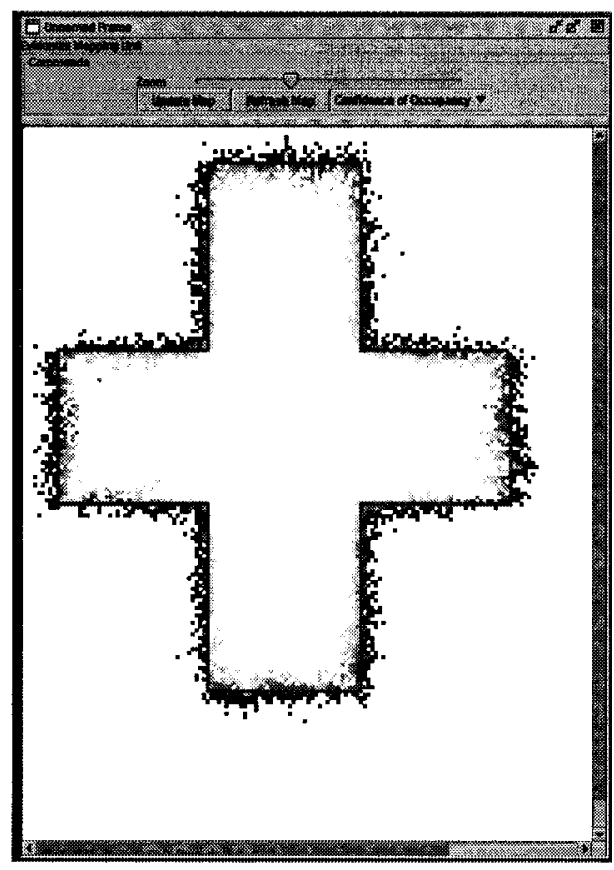

5.4c) Occupancy Plausibility

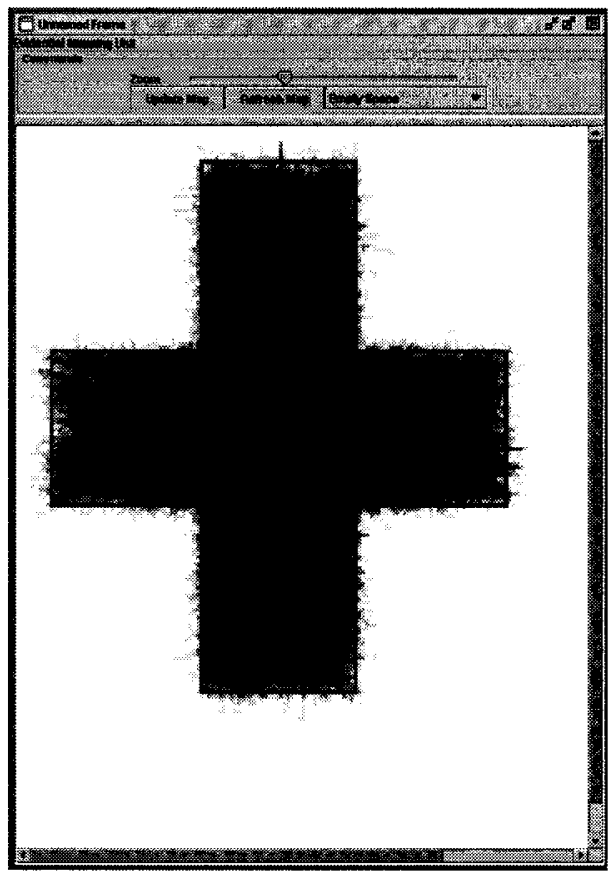

5.4b) Emptiness Support

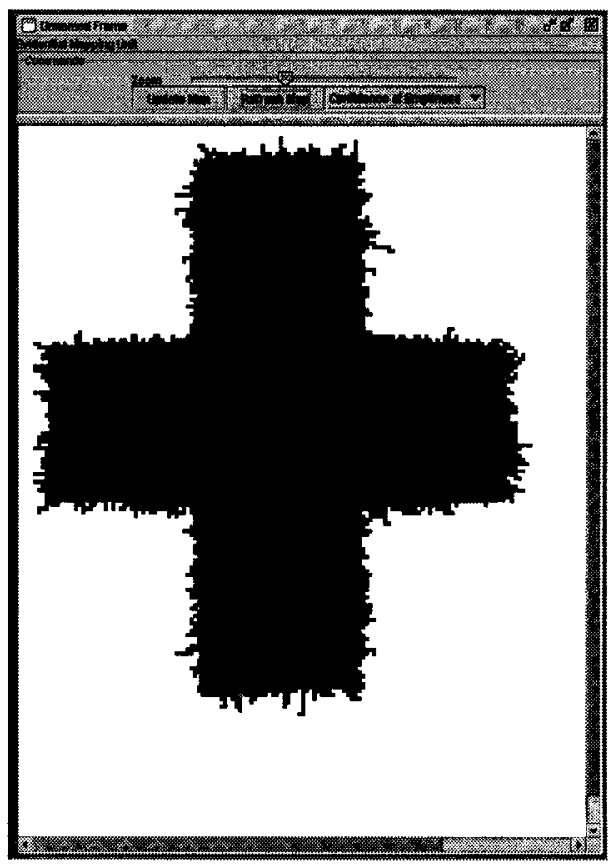

5.4d) Emptiness Plausibility

Figure 5.4: Maps Created by Test Case 1-1 

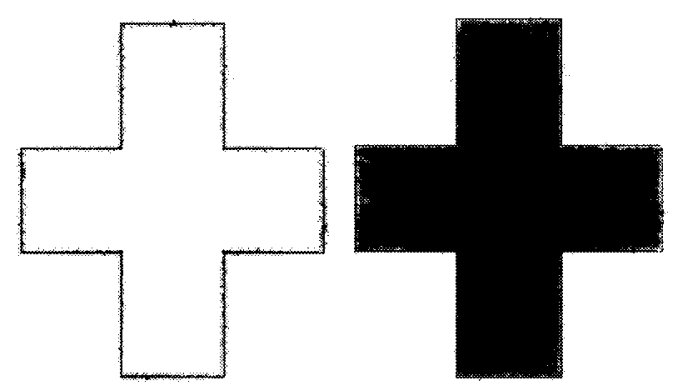

5.5b) Emptiness

5.5a) Occupancy Support

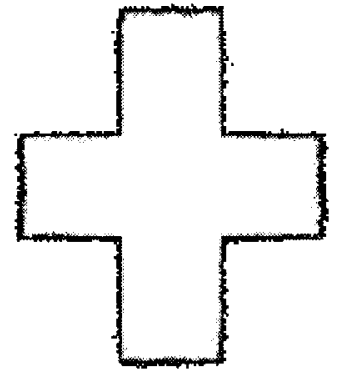

5.5c) Occupancy Plausibility

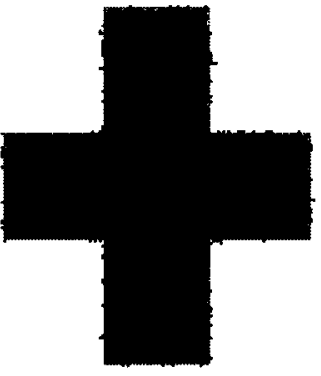

5.5d) Emptiness Plausibility

Figure 5.5: Maps Created by Test Case 1-2

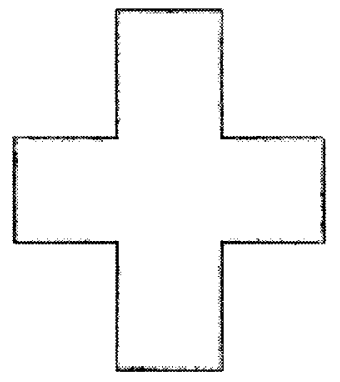

5.6a) Occupancy Support

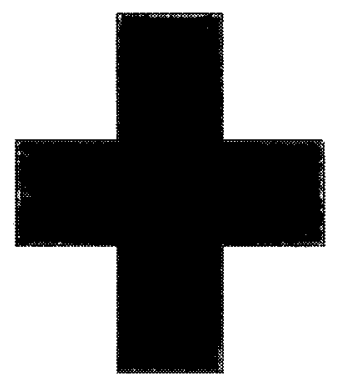

5.6b) Emptiness Support

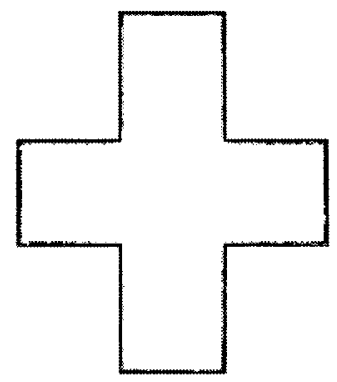

5.6c) Occupancy Plausibility

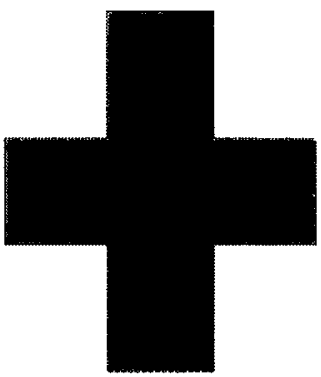

5.6d) Emptiness Plausibility

Figure 5.6: Maps Created by Test Case 1-3

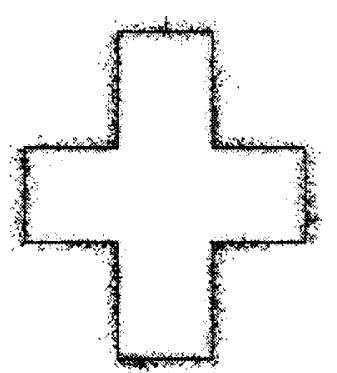

5.7a) Occupancy Support

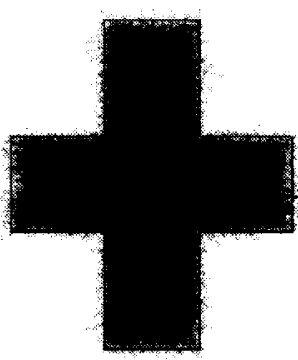

5.7b) Emptiness Support

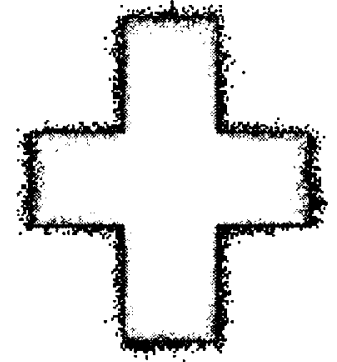

5.7c) Occupancy Plausibility

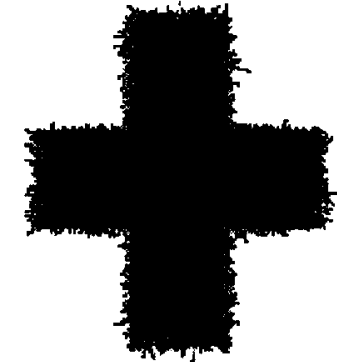

5.7d) Emptiness Plausibility

Figure 5.7: Maps Created by Test Case 2-1 


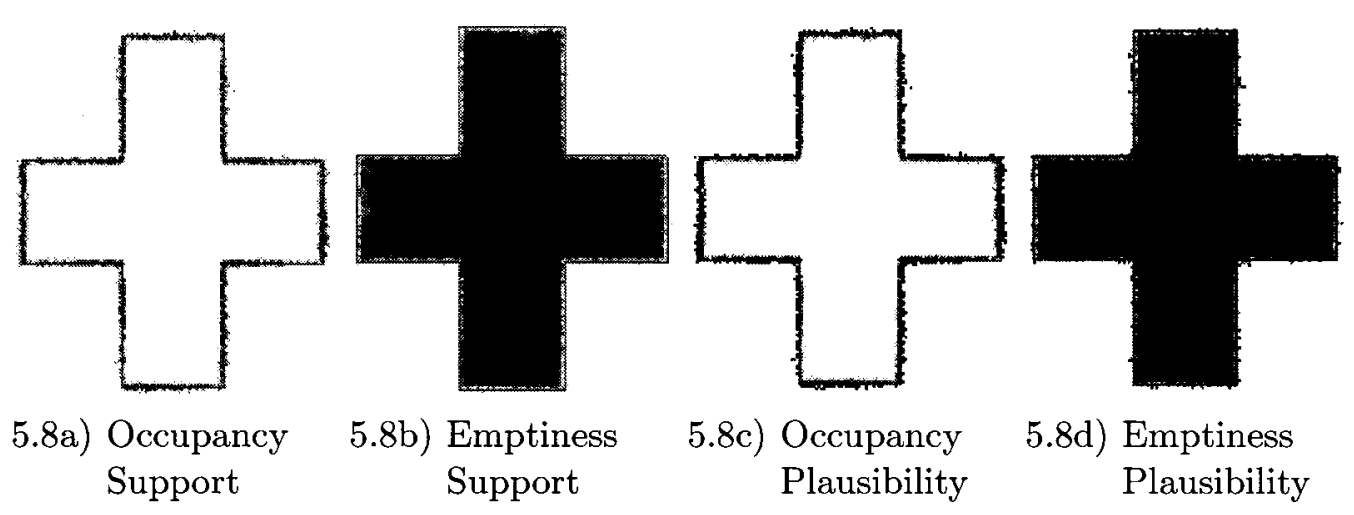

Figure 5.8: Maps Created by Test Case 2-2

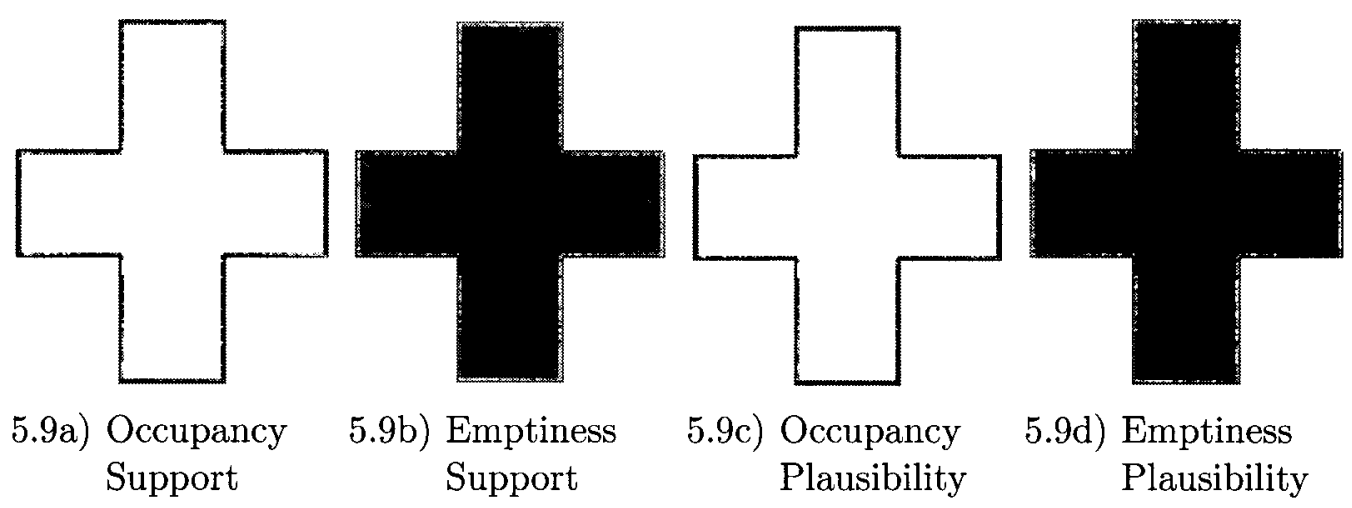

Figure 5.9: Maps Created by Test Case 2-3 


\section{Low Occupancy Support and Moderate Emptiness Support}

Test cases 3-1, 3-2, and 3-3 correspond to cases with low occupancy and moderate emptiness support for varying amounts of sensor noise. The support for emptiness bleeds into occupied areas near the boundary. These are the converse case of the previous section, and are more noticeable than the results of the previous section. They are more noticeable than the converse cases 2-1, 2-2, and 2-3 because empty cells tend to get more support overall due to the sensor model, as described earlier.

\section{High Occupancy Support and Low Emptiness Support}

Test cases 4-1, 4-2, and 4-3 correspond to cases with high occupancy and low emptiness support for varying amounts of sensor noise. These parameters are chosen to mitigate the effect of empty cells getting more overall support than the occupied cells. As can be seen in Figure 5.13c, for example, the bleeding of occupancy into empty space is now more pronounced than in the previous cases.

\section{Low Occupancy Support and High Emptiness Support}

Test cases 5-1, 5-2, and 5-3 correspond to cases with low occupancy and high emptiness support for varying amounts of sensor noise. The maps created with these parameters are similar to those created with low occupancy support and moderate emptiness support. This is because empty cells tend to get more emptiness support overall due to the laser sensor model, and the emptiness support for cells reach their limit of 1 . The difference between the two cases can only be seen during runtime, where maps created with high emptiness support reach the maximum emptiness support value of 1 sooner than the maps created with moderate emptiness support. 


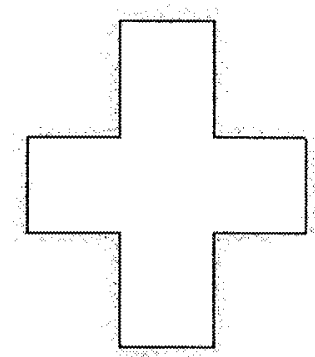

5.10a) Occupancy Support

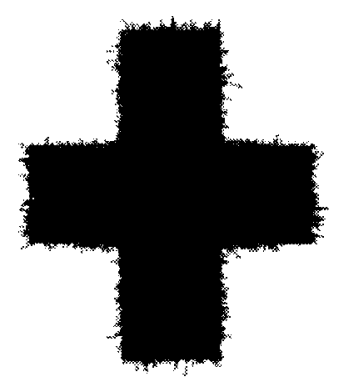

5.10b) Emptiness Support

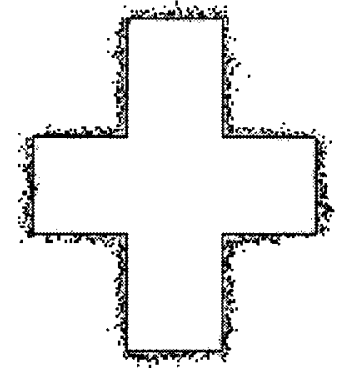

5.10c) Occupancy Plausibility

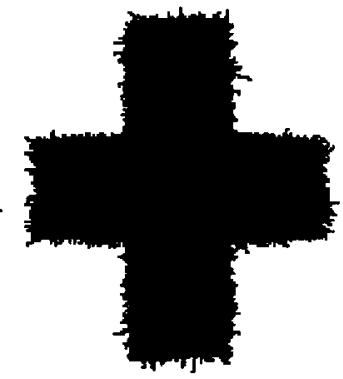

5.10d) Emptiness Plausibility

Figure 5.10: Maps Created by Test Case 3-1

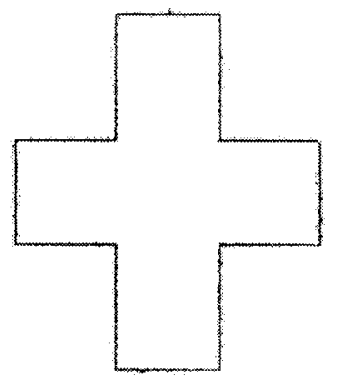

5.11a) Occupancy Support

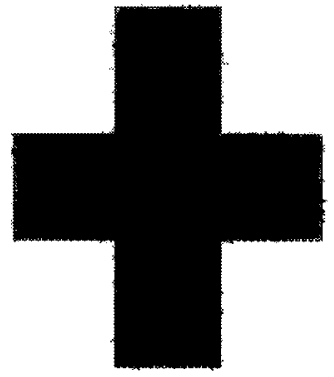

5.11b) Emptiness Support

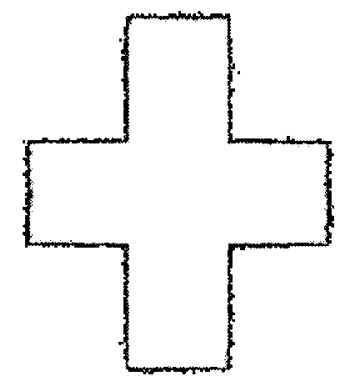

5.11c) Occupancy Plausibility

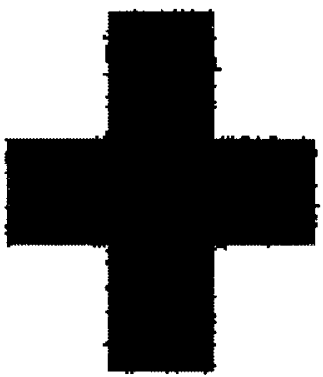

5.11d) Emptiness Plausibility

Figure 5.11: Maps Created by Test Case 3-2

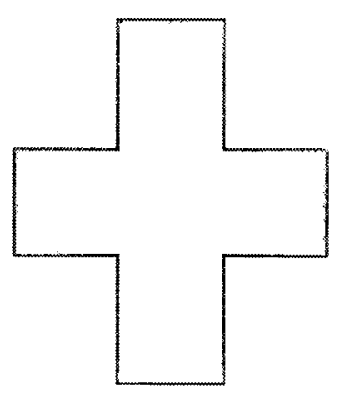

5.12a) Occupancy Support

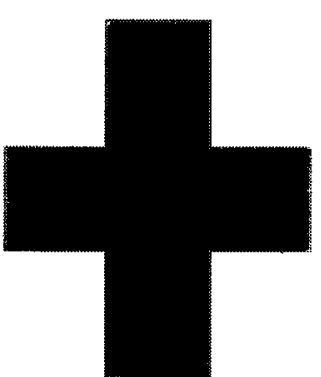

5.12b) Emptiness Support

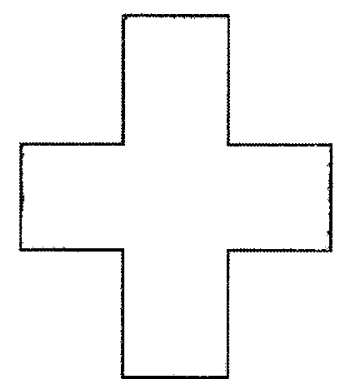

5.12c) Occupancy Plausibility

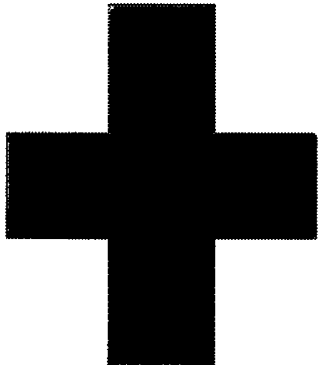

5.12d) Emptiness Plausibility

Figure 5.12: Maps Created by Test Case 3-3 


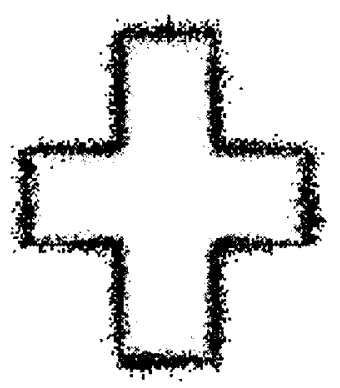

5.13a) Occupancy Support

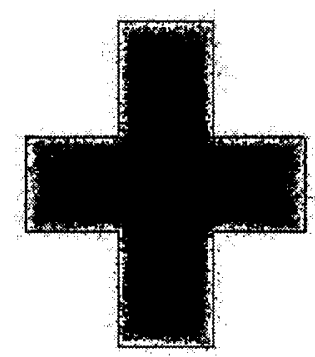

5.13b) Emptiness Support

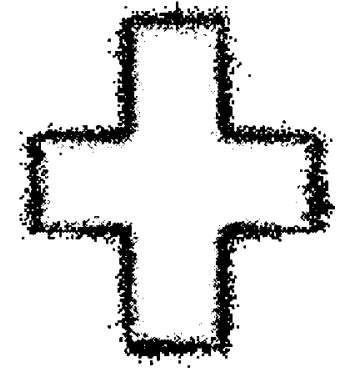

5.13c) Occupancy Plausibility

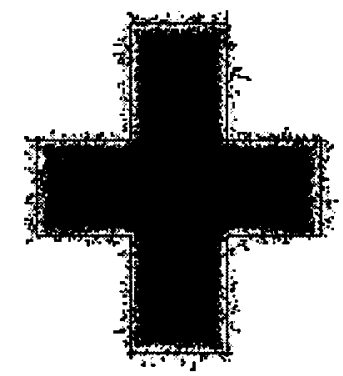

5.13d) Emptiness Plausibility

Figure 5.13: Maps Created by Test Case 4-1

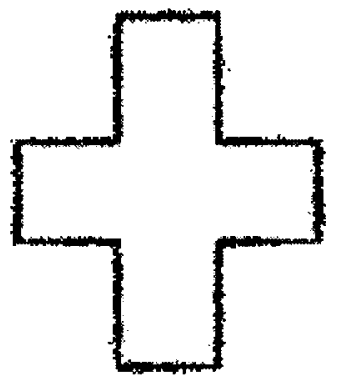

5.14a) Occupancy Support

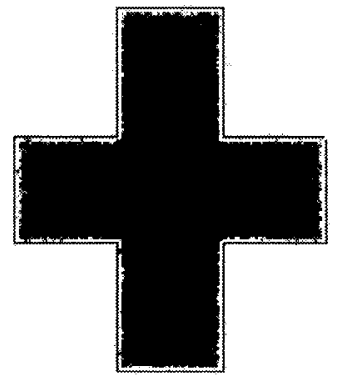

5.14b) Emptiness

Support

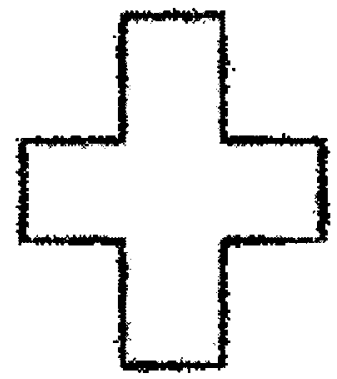

5.14c) Occupancy Plausibility

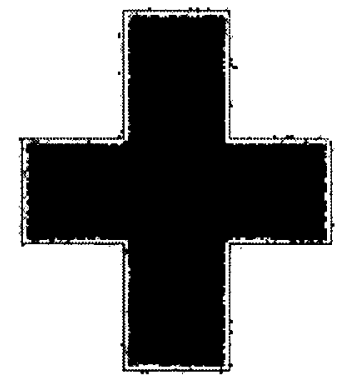

5.14d) Emptiness Plausibility

Figure 5.14: Maps Created by Test Case 4-2

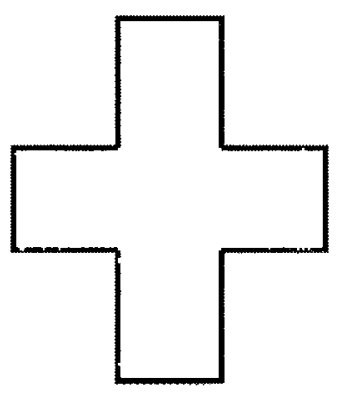

5.15a) Occupancy Support

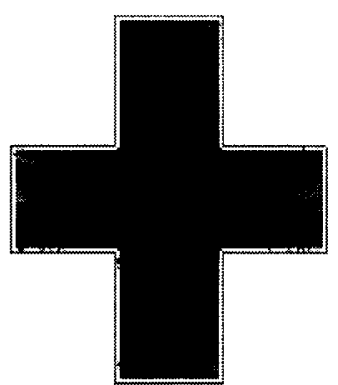

5.15b) Emptiness Support

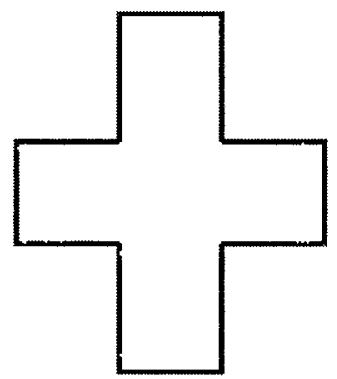

5.15c) Occupancy Plausibility

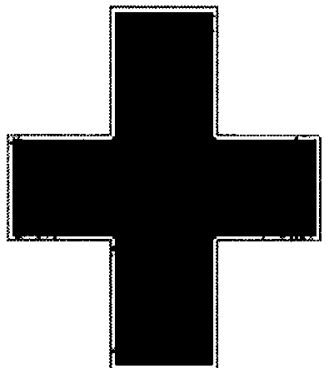

5.15d) Emptiness Plausibility

Figure 5.15: Maps Created by Test Case 4-3 


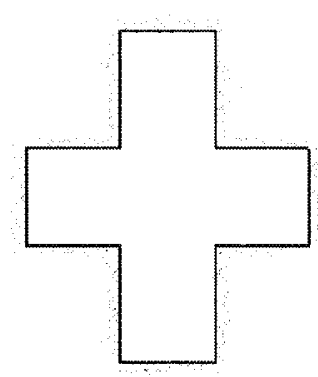

5.16a) Occupancy Support

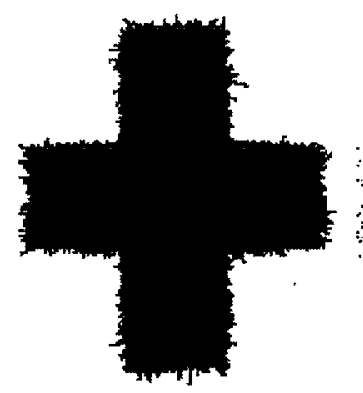

5.16b) Emptiness Support

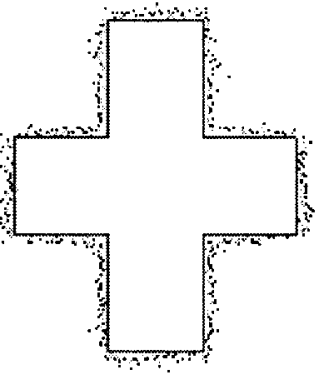

5.16c) Occupancy Plausibility

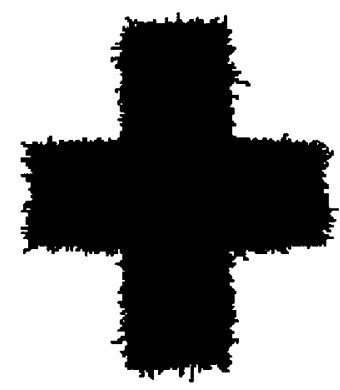

5.16d) Emptiness Plausibility

Figure 5.16: Maps Created by Test Case 5-1

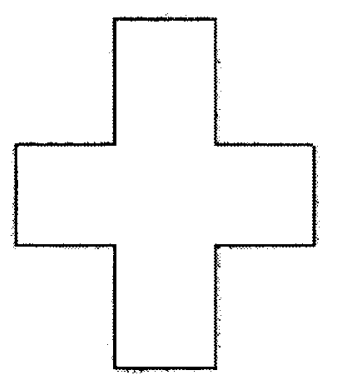

5.17a) Occupancy Support

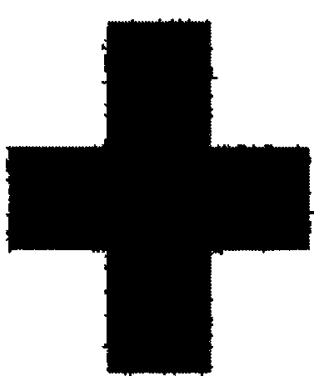

5.17b) Emptiness Support

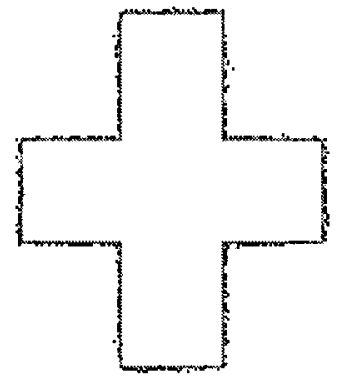

5.17c) Occupancy Plausibility

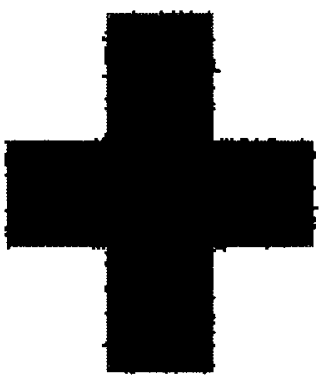

5.17d) Emptiness Plausibility

Figure 5.17: Maps Created by Test Case 5-2

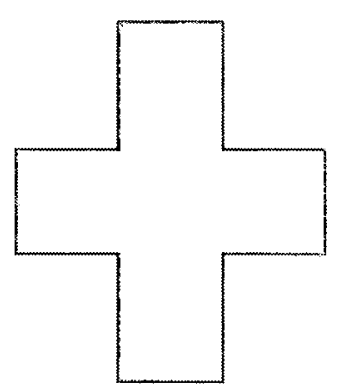

5.18a) Occupancy Support

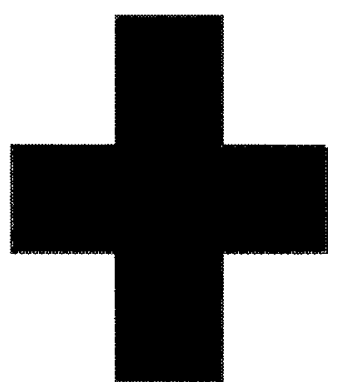

5.18b) Emptiness Support

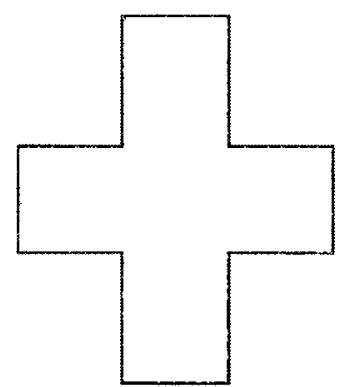

5.18c) Occupancy Plausibility

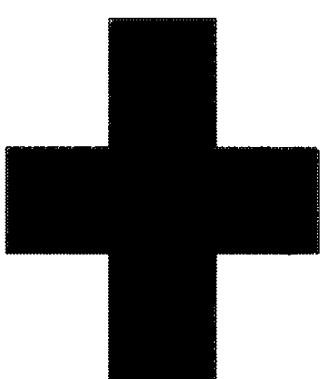

5.18d) Emptiness Plausibility

Figure 5.18: Maps Created by Test Case 5-3 


\section{High Support for Occupancy and Emptiness}

Test cases 6-1, 6-2, and 6-3 correspond to cases with high occupancy and emptiness support for varying amounts of sensor noise. The maps created from these test cases are "crisp" and distinct; that is to say, there are no grey areas in the map, indicating uncertainty. As such, the maps illustrated using the support measure are the same as the maps illustrated using the plausibility measure.

From the point of view of Dempster-Shafer theory, the combination of sensor evidence that are highly supportive of their respective proposition virtually eliminates unassigned support, ie: $\operatorname{Bel}(\{O E\}) \approx 0$.

From a mathematical point of view, these test cases closely resemble the results that would be generated from the use of the Bayesian merge since only a small value of 0.01 is assigned to $m(\{O E\})$. That small value is virtually inconsequential to the calculations, and the high amount of support for propositions makes it resemble the case whereby complete knowledge of all the probabilities involved are known.

These test cases perform well for cases where there is low sensor noise. In the case where sensor noise is set to $0.1 \mathrm{~m}$, Figure $5.21 \mathrm{c}$ corresponds almost exactly to the ideal occupancy map, while Figure 5.21d corresponds just as closely to the ideal emptiness map.

For the case where sensor noise is set to the high value of $0.5 \mathrm{~m}$, it can be seen in Figure 5.19 that although the created map is highly confident of its results, the emptiness map is inaccurate in that it "bleeds" into the occupied space.

These test cases specify parameters for the laser sensor model that accentuates the fallacy in using Dempster-Shafer theory with highly contradictory evidence, as described in Section 3.1.4. As such, cell states are updated in a manner such that cells have high numerical values for either occupancy or emptiness, without much 
uncertainty involved. Therefore, the advantage of being able to model the unknown, the greatest strength of Dempster-Shafer theory, is essentially taken away due to the chosen parameter values. In this case, the model is putting little weight on past evidence, and a large amount of weight on the newest piece of evidence.

\section{Absolute Support for Occupancy and Emptiness}

Test case 7 is a special test that assigns absolute certainty to occupancy and emptiness support. This test case is only valid for the case where there is no sensor noise. Cases with sensor noise create contradictory evidence, and Dempster-Shafer theory fails for cells with contradictory evidence. This again emphasizes the fallacy in using Dempster-Shafer theory with highly contradictory evidence, as described in Section 3.1.4. In this case, the different pieces of evidence are flatly contradictory, and the Dempster-Shafer method fails completely.

\subsubsection{Office Map Test Cases}

With the results of the symmetric map test cases, the parameter values $\lambda_{1}=0.8$, $\lambda_{2}=0.1$ appeared to provide the best results even in a great amount of sensor error. These values are chosen for further testing in the larger office map test environment described in Section 5.1.2. In this test case, the sensor noise is set to $0.5 \mathrm{~m}$. The results are shown in Figure 5.22, Figure 5.23, Figure 5.24, and Figure 5.25. As can be seen, the created maps resemble the environment quite well. With this high level of sensor noise, however, occupied cells are mistakenly labeled as empty as seen in Figure 5.25. 

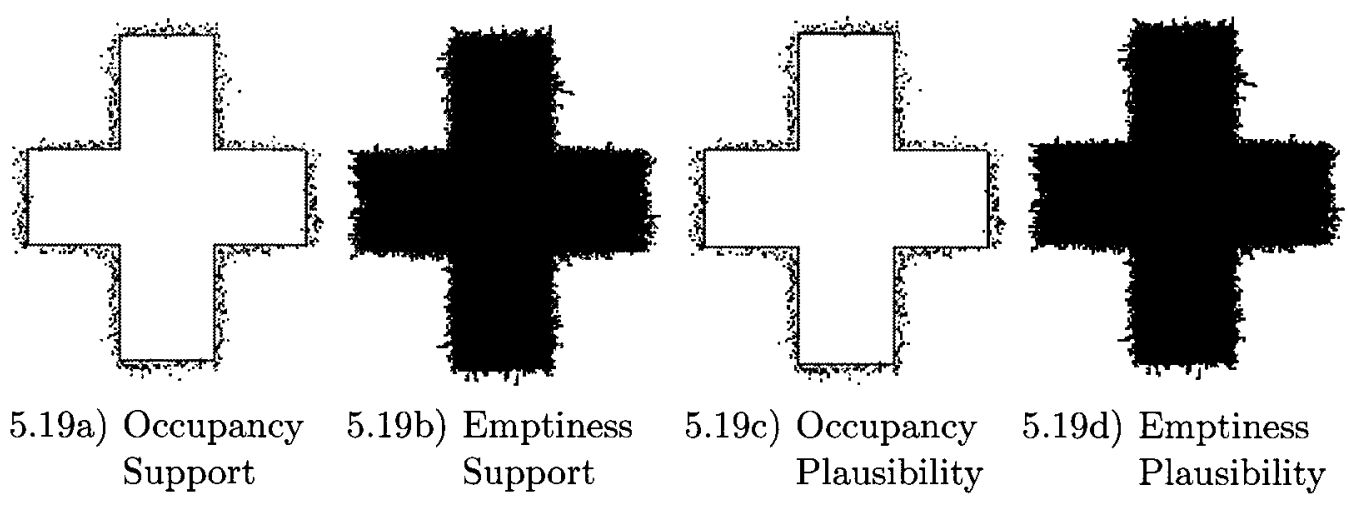
5.19c) Occupancy 5.19d) Emptiness Plausibility Plausibility

Figure 5.19: Maps Created by Test Case 6-1

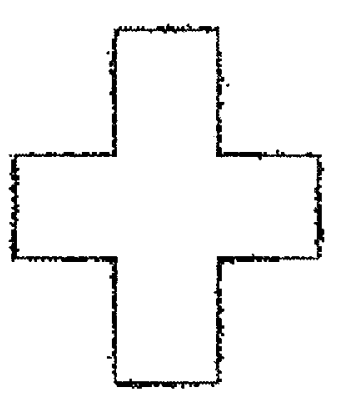

5.20a) Occupancy Support

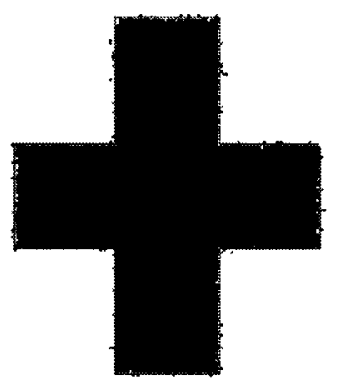

5.20b) Emptiness

Support

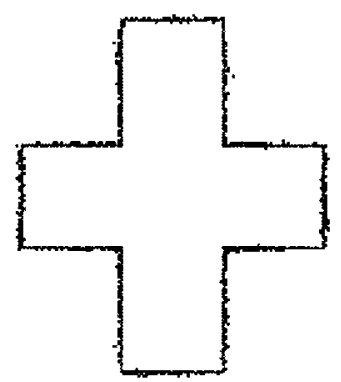

5.20c) Occupancy Plausibility

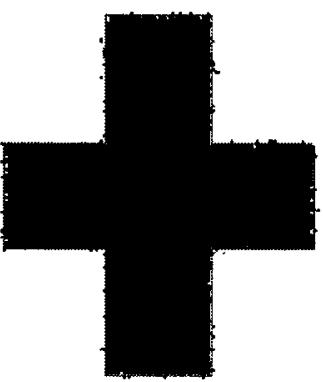

5.20d) Emptiness Plausibility

Figure 5.20: Maps Created by Test Case 6-2

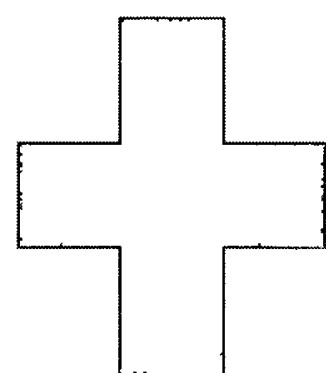

5.21a) Occupancy Support

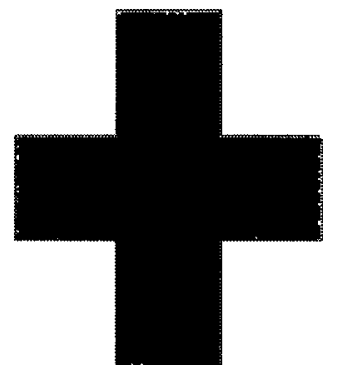

5.21b) Emptiness Support

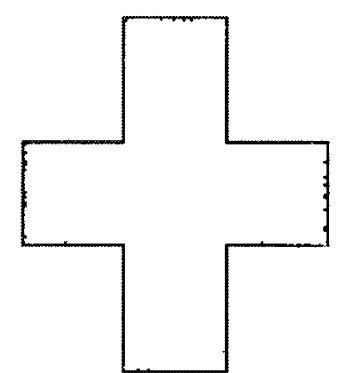

5.21c) Occupancy Plausibility

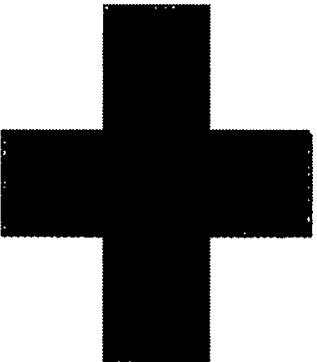

5.21d) Emptiness Plausibility

Figure 5.21: Maps Created by Test Case 6-3 


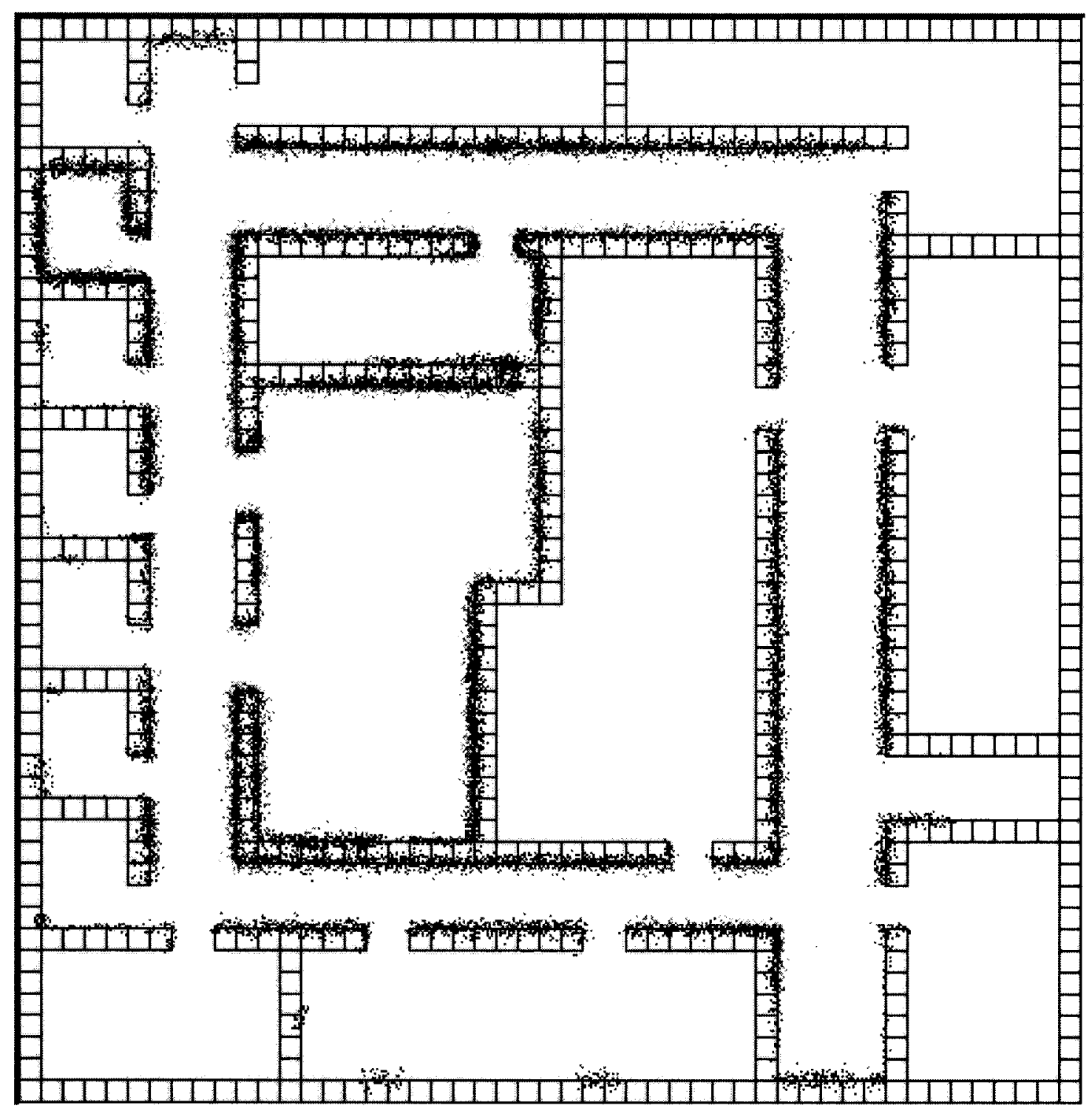

Figure 5.22: Occupancy Support Map Created Following Office Mapping Test Case 1 


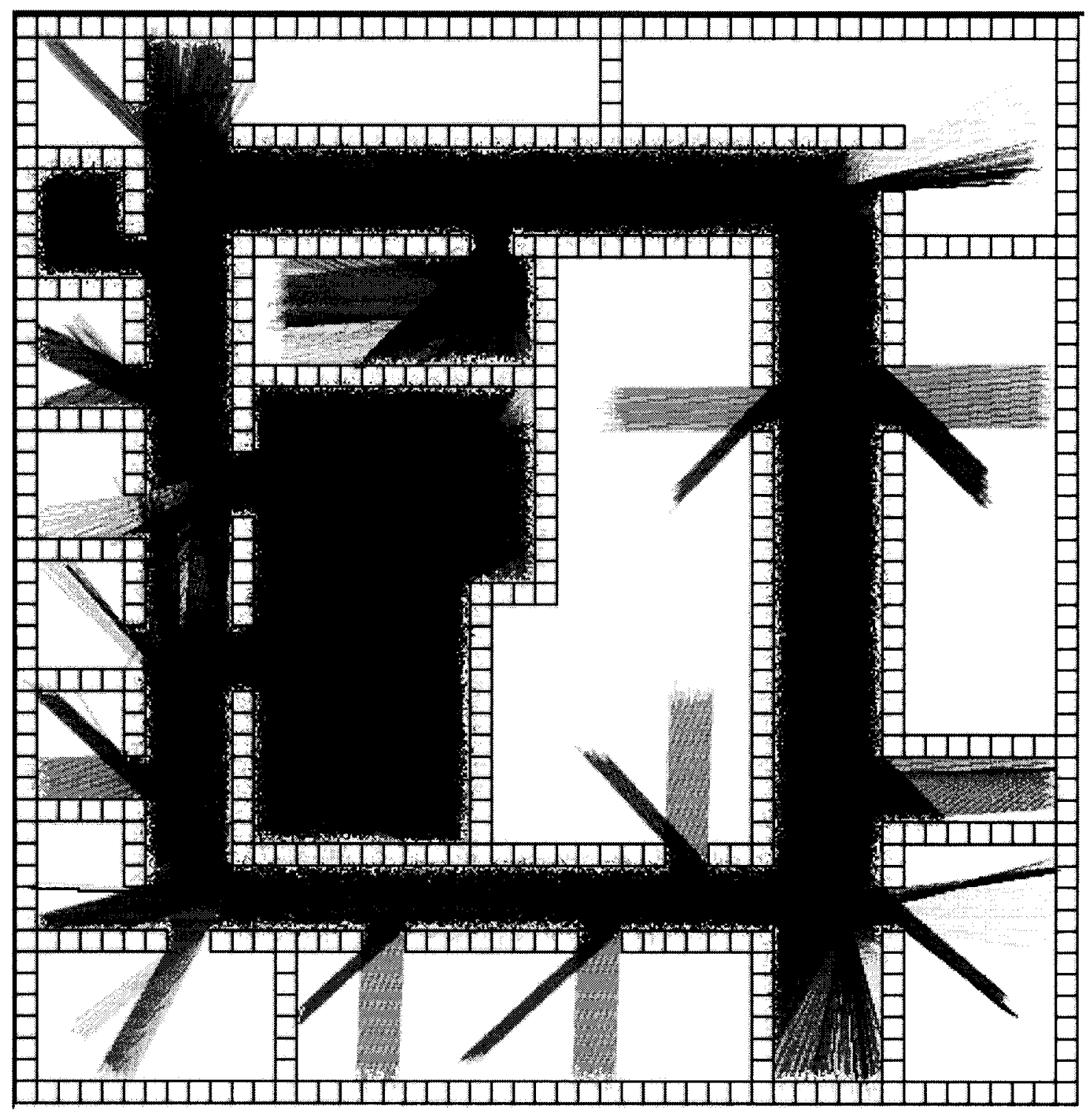

Figure 5.23: Emptiness Support Map Created Following Office Mapping Test Case 1 


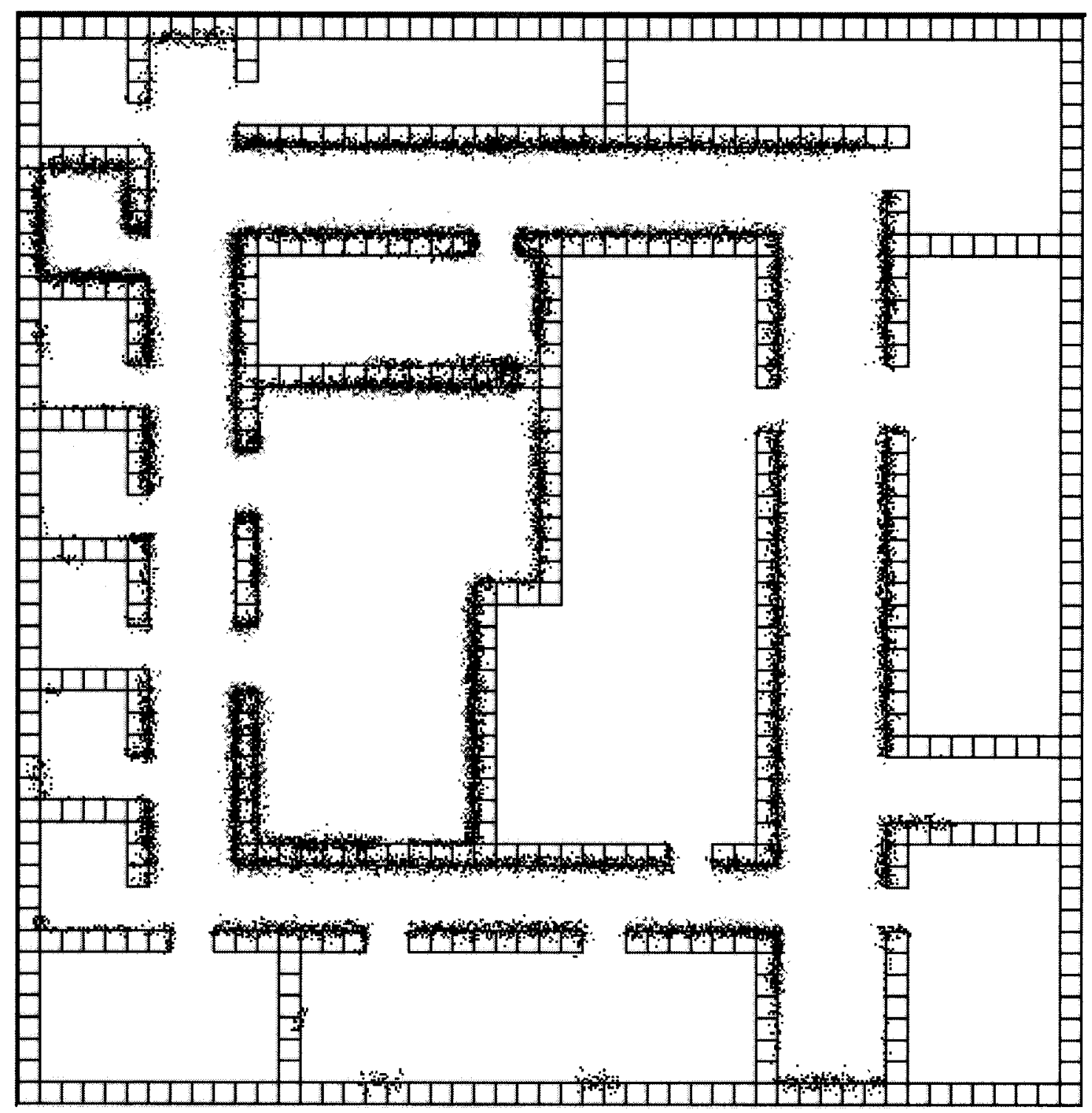

Figure 5.24: Occupancy Plausibility Map Created Following Office Mapping Test Case 1 


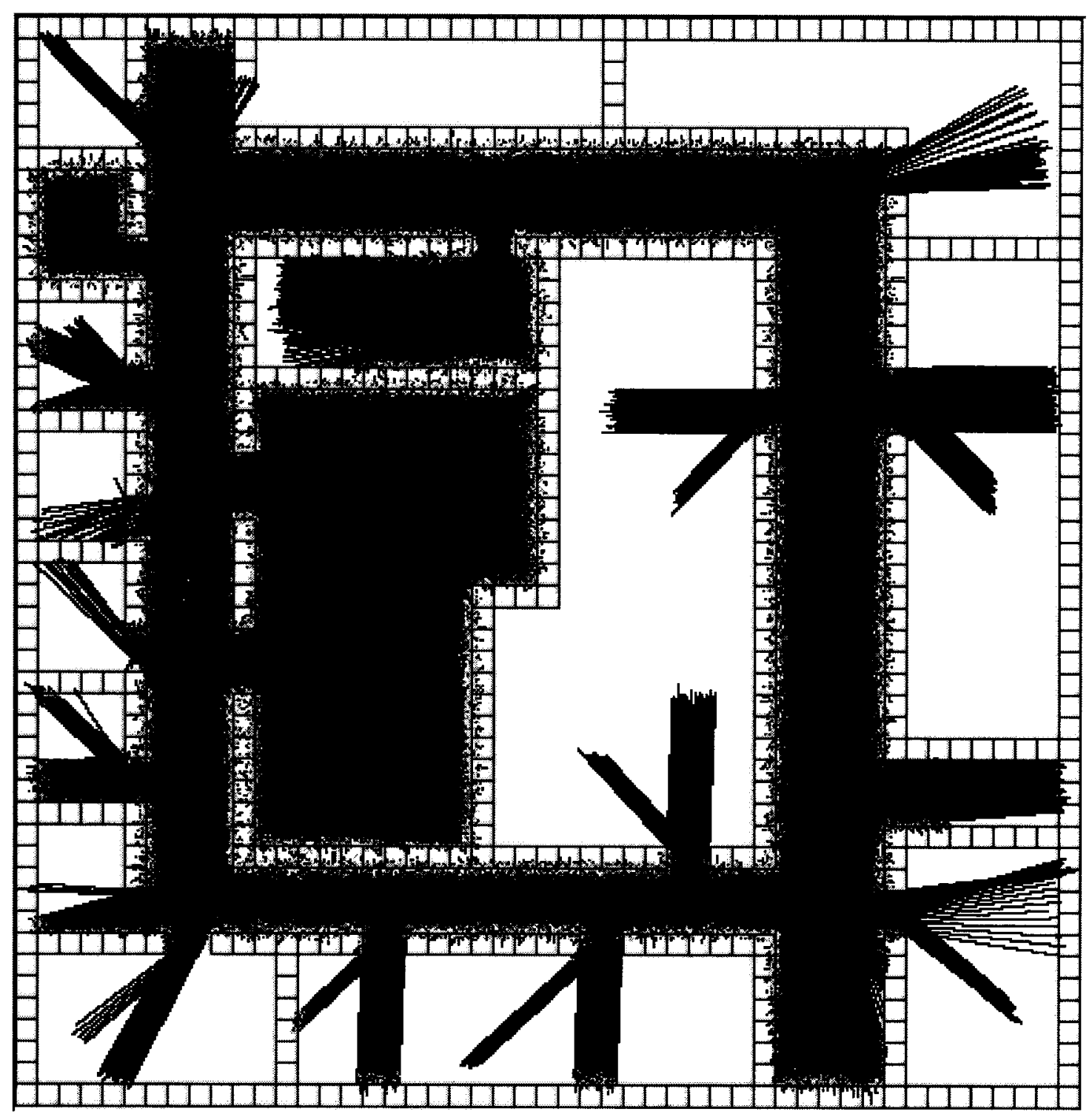

Figure 5.25: Emptiness Plausibility Map Created Following Office Mapping Test Case 1 


\subsubsection{Discussion}

As can be seen, varying the parameters of the laser sensor model does affect the map created by the robot using noisy sensor observations. The map's general shape, however, is re-created by the mapping method. In most cases, it is at the boundaries between occupied space and free space that there is any noticeable difference between using the different laser sensor model parameters. That is also the area that concerns the robot most, as knowing the occupancy boundaries allows the robot to plan collision-free paths through the environment. In general, it is better for a robot to believe that an empty cell is occupied, rather than the converse case. A robot does not encounter collisions by avoiding an empty area that is mistakenly labeled as being occupied, but it does encounter collisions by entering an occupied area that is mislabeled as being empty.

The level of noise is most problematic for the different maps that are created. Low to medium levels of sensor noise can be dealt with by choosing a high level of support for occupied cells, and a low level of support for empty cells. Doing so allows the mapping method to err on the side of occupancy, and empty cells near the boundary may be mislabeled as being occupied which is more desirable than having mislabeled occupied cells.

With high levels of sensor noise, however, this solution does not work perfectly. Occupied cells relatively far away from the boundary are mislabeled as being empty, as seen in cases with high noise such as Figure 5.4d and Figure 5.25. These mislabeled cells, however, are not corrected due to the point based nature of the laser range sensor. For these mislabeled cells to be corrected, mislabeled cells past the occupancy boundary need to be given occupancy support, which is not possible with the laser sensor model used. 
In such noisy conditions, however, the maps created are not completely useless. For the office map test case with large amounts of noise, the occupancy plausibility map (Figure 5.24) may still be used for path planning purposes. In this map, it is more likely for an empty cell to be mislabeled as being occupied, than an occupied cell being mislabeled as being empty, therefore allowing the robot to avoid collisions.

Another possibility to use the maps created under noisy conditions is to insert a separate post-processing step to correct mislabeled cells based on the state of surrounding cells, or some other heuristics.

A general observation from the mapping test cases is that as sensor noise is reduced, there is less need for evidential theory. With less noise comes less uncertainty to reason about, therefore the maps created then resembles a true map. Also, it is not encouraged to use near absolute evidence values for the laser sensor model as it reduces the advantage of using Dempster-Shafer theory, or even makes DempsterShafer unworkable in the case of absolute evidence values. If the evidence from the laser is expected to be close to the absolute truth, other simpler methods of evidence combination may be acceptable, such as averaging, or simply taking the latest piece of evidence.

\subsection{Localisation}

The de facto standard to robot localisation, the Monte Carlo Localisation (MCL) method is the focus of localisation experiments. MCL and its variants, the Clustered Particle Filtering MCL (CPF-MCL), the Uniform MCL (U-MCL), and the Uniform Clustered Particle Filtering MCL (UCPF-MCL) are implemented in the simulator and tested under different criteria. The implemented methods globally localise without 
any a priori information about robot location, and upon successful localisation, tracks the robot location through the environment until the robot location is lost. If this is the case, relocalisation is required, in which case global localisation is again started with a uniform distribution to relocalise the robot. The following results are of interest:

- Sensitivity to sample size used, and overall computational complexity;

- Sensitivity to environmental attributes such as symmetry, and size;

- Sensitivity to sensor and motion noise.

\subsubsection{Symmetric Map Test Cases}

Following Section 3.2, the sole adjustable parameter of the MCL and U-MCL methods is the sample size for the filter. The clustering methods, such as the CPF-MCL, and the UCPF-MCL, have different parameters listed below:

- Number of initial samples seeded in the filter before clustering process

- Number of steps before clustering process

- Number of samples for each cluster after clustering process

- Number of clusters to keep

The filter specific parameters are combined with environmental parameters, sensor and motion noise, to create the test cases listed in Table 5.2. The test cases are run using the symmetric map test environment described in Section 5.1.2.

The results of the test cases are in Appendix B,and are analysed with the following criteria: 
Table 5.2: Localisation Test Cases and Corresponding Results for Varying Parameters

\begin{tabular}{|c|c|c|c|c|c|}
\hline $\begin{array}{c}\text { Test Case } \\
\# \\
\end{array}$ & $\begin{array}{c}\text { Sample } \\
\text { Size }\end{array}$ & $\begin{array}{c}\text { Cluster } \\
\text { Sample Size } \\
\end{array}$ & $\begin{array}{c}\text { Sensor } \\
\text { Noise (m) }\end{array}$ & $\begin{array}{c}\text { Motion } \\
\text { Noise (\%) }\end{array}$ & Results \\
\hline MCLx-1 & variable & & 0.1 & \multirow{3}{*}{10} & Table B-1, Table B-10, Table B-19 \\
\hline MCLx-2 & variable & & 0.3 & & Table B-2, Table B-11, Table B-20 \\
\hline MCLx-3 & variable & & 0.5 & & Table B-3, Table B-12, Table B-21 \\
\hline MCLX-4 & variable & & 0.1 & \multirow{3}{*}{30} & Table B-6, Table B-15, Table B-24 \\
\hline MCLx-5 & variable & & 0.3 & & Table B-4, Table B-13, Table B-22 \\
\hline MCLx-6 & variable & & 0.5 & & Table B-5, Table B-14, Table B-23 \\
\hline MCLx-7 & variable & & 0.1 & \multirow{3}{*}{50} & Table B-7, Table B-16, Table B-25 \\
\hline MCLx-8 & variable & & 0.3 & & Table B-8, Table B-17, Table B-26 \\
\hline MCLx-9 & variable & & 0.5 & & Table B-9, Table B-18, Table B-27 \\
\hline$\overline{\mathrm{CPF}-1}$ & $\overline{100,000}$ & 1000 & $\overline{0.1}$ & \multirow{3}{*}{10} & Table B-28 \\
\hline $\mathrm{CPF}-2$ & 100,000 & 1000 & 0.3 & & Table B-29 \\
\hline $\mathrm{CPF}-3$ & 100,000 & 1000 & 0.5 & & Table B-30 \\
\hline CPF-4 & 100,000 & 1000 & 0.1 & \multirow{3}{*}{30} & Table B-33 \\
\hline CPF-5 & 100,000 & 1000 & 0.3 & & Table B-31 \\
\hline CPF-6 & 100,000 & 1000 & 0.5 & & Table B-32 \\
\hline CPF-7 & 100,000 & 1000 & 0.1 & \multirow{3}{*}{50} & Table B-34 \\
\hline $\mathrm{CPF}-8$ & 100,000 & 1000 & 0.3 & & Table B-35 \\
\hline CPF-9 & 100,000 & 1000 & 0.5 & & Table B-36 \\
\hline$\overline{\text { UMCL }}$ & 100,000 & & 0.5 & $\overline{50}$ & \\
\hline UCPF-1 & 100,000 & 1000 & $\overline{0.1}$ & \multirow{3}{*}{10} & Table B-28 \\
\hline$\overline{\text { UCPF-2 }}$ & 100,000 & 1000 & 0.3 & & Table B-29 \\
\hline UCPF-3 & 100,000 & 1000 & 0.5 & & Table B-30 \\
\hline UCPF-4 & 100,000 & 1000 & 0.1 & \multirow{3}{*}{30} & Table B-33 \\
\hline UCPF-5 & 100,000 & 1000 & 0.3 & & Table B-31 \\
\hline UCPF-6 & 100,000 & 1000 & 0.5 & & Table B-32 \\
\hline UCPF-7 & 100,000 & 1000 & 0.1 & \multirow{3}{*}{50} & Table B-34 \\
\hline UCPF-8 & 100,000 & 1000 & 0.3 & & Table B-35 \\
\hline UCPF-9 & 100,000 & 1000 & 0.5 & & Table B-36 \\
\hline
\end{tabular}

All clustering algorithms cluster after the first step.

All clustering algorithms keep a maximum of 8 clusters.

For brevity, the test cases of the format MCLx-1 through MCLx-9 specify the same test case, but with differing initial sample sizes. The sample sizes are 100,000 samples for $x=1$, 10,000 for $\mathrm{x}=2$, and 1,000 for $\mathrm{x}=3$. ie: MCL2-5 is test case 5 using 10,000 samples. 
- Criterion 1: The true location of the robot is found,

- Criterion 2: Distinguishable similar location of the robot is found,

- Criterion 3: Other possible similar locations of the robot are found.

The criteria considered here all refer to the localisation method's ability to create clusters of samples representing the robot's location at the different locations shown in Figure 5.26a.

Criterion 1 is normally the best way to rate localisation techniques. Suppose the robot is truly at location 1 . Then, a localisation method passes criterion 1 if it is able to create a cluster of particles in that vicinity. Due to the symmetric layout of the environment, however, there are further criteria to be considered. Using simply range observations and odometry, all 4 locations shown are essentially the same. It is not possible for the robot to determine which location it is at due to the lack of distinguishing features. This is not an unrealistic environment; building architecture might use symmetry for aesthetic reasons and the corridors must be distinguished uniquely using other means in addition to the physical layout of the environment.

Criterion 2 deals with distinguishable similar locations, such as location 2. This location is distinguishable in that it is dissimilar to location 3 and 4 at certain locations. More specifically, such locations are locations that are similar to other spots at one location, but distinct from those other locations given further robot observations and actions. Suppose the true robot location is again at location 1 in Figure 5.26a. All other locations shown are possible. Upon further robot action and observation, the robot arrives at the situation depicted in Figure 5.26b. Locations 1 and 2 both remain equally possible, but locations 3 and 4 are distinctly impossible, as that suggests the robot is implanted within a wall. This is due to the fact that although the test 
environment appears at first glance to contain four identical corridors, the corridors are actually of different length. The vertical corridors are one metre longer than the horizontal corridors. Location 1 does not belong under this criterion, however, since it belongs under criterion 1.

Criterion 3 deals with the other indistinguishable similar locations shown, namely, locations 3 and 4 . When the robot is in the middle of the test environment, all 4 locations are indistinguishable, and the localisation method should be unable to tell them apart. However, location 1 and location 2 belong to criteria 1 and 2 respectively, thus the two remaining similar locations belong to criterion 3 .

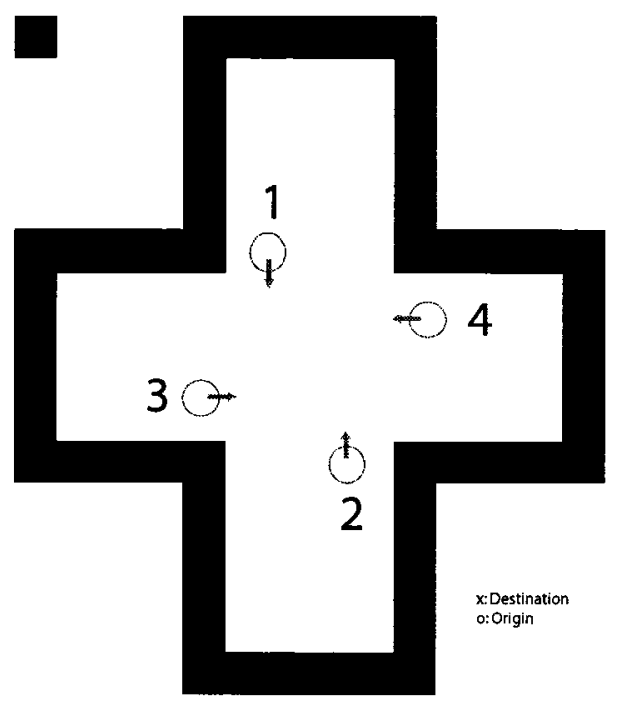

5.26a) Similar Locations

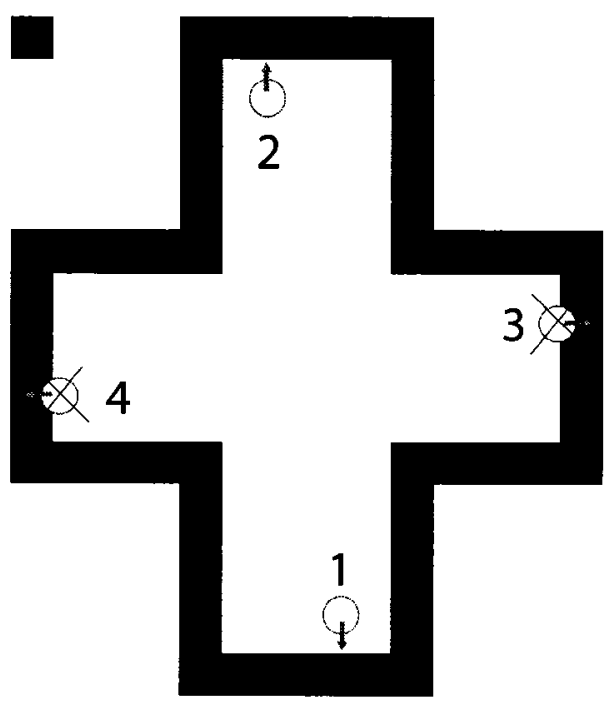

5.26b) Distinguished Similar Locations

Figure 5.26: Illustration of Similar Locations and Distinguished Similar Locations

Table 5.3, Table 5.4, and Table 5.5 summarise the results of using the MCL method using different numbers of samples. The following are observed:

- As sample size for the filter is decreased, the general trend is an increased amount of global relocalisation. For MCL1 test cases, the number of relocalisa- 
tions is typically a maximum of 1 , whereas for MCL3 test cases, the number of relocalisations is as high as 6 . This is expected due to the decreased effectiveness of representing continuous quantities with discrete Monte Carlo sampling with smaller sample size.

- As the amount of sensor and motion error is decreased, the general trend is an increased amount of relocalisation. This is shown in Table 5.5 for test cases MCL3-1, MCL3-4, and MCL3-7. Although this result is counter intuitive, that the method decreases in performance as sensors and actuators models get more accurate, this is well documented in [127]. It is advantageous to use the most accurate sensors possible, but the sensor and action models cannot be chosen to be too accurate. As sensor and action models get too accurate, they are unable to robustly inject samples into the filter to counteract the effects of non-ideal Monte Carlo sampling and inaccurate localisation.

- The MCL method is usually able to distinguish many of the possible locations of the robot during the initial stages of the localisation process, like in Figure 5.27a. The filter, however, ends up selecting just one of the possible locations at the end of the robot's movement. In Figure 5.27b, the filter already lost track of 2 other similar clusters after just 4 localisation attempts.

Table 5.6 summarises the results of using the new CPF-MCL method proposed in this thesis. The following are observed:

- CPF-MCL is able to find all similar locations in all test cases.

- CPF-MCL finds the similar locations in all cases except where there are low sensor and motion noise. In such test cases, CPF-MCL typically finds the similar 


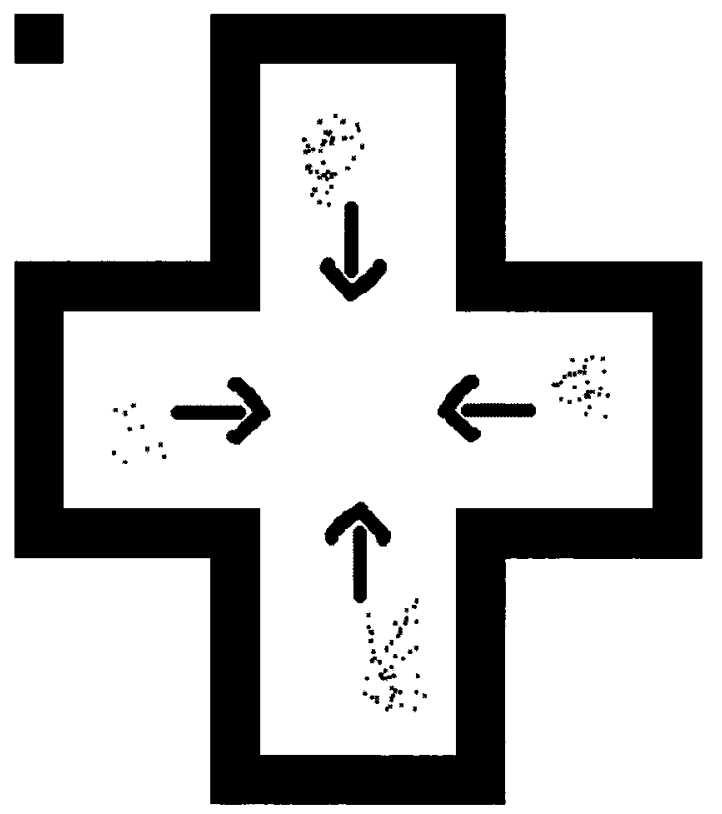

5.27 a) Step 1

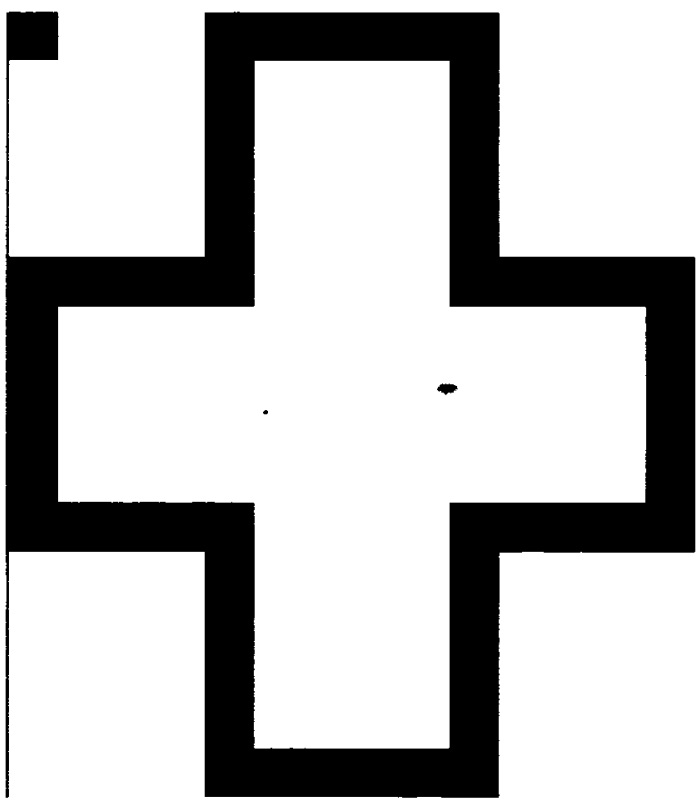

$5.27 \mathrm{~b})$ Step 4

Figure 5.27: Example of MCL Settling on One Possible Location from Test Case MCL1-2

locations when the robot is in the center of the environment, where the similar locations cannot be distinguished from one another, shown in Figure 5.28a. Only the distinguishable similar locations are kept when the robot moves into the corridors of unequal length, like shown in Figure 5.28b. The CPF-MCL is able to inject clusters of samples when the robot is in the centre of the environment because it has a background task that constantly tries to add new possible clusters without regard to past robot observations and actions.

There are no successful results for U-MCL. Figure 5.29 shows the first execution of the U-MCL method for the U-MCL test case. As can be seen, the resampling stage as defined for the U-MCL method does not work for a general environment where environmental similarities exist, where the U-MCL filter contains multiple modes, as expected. 

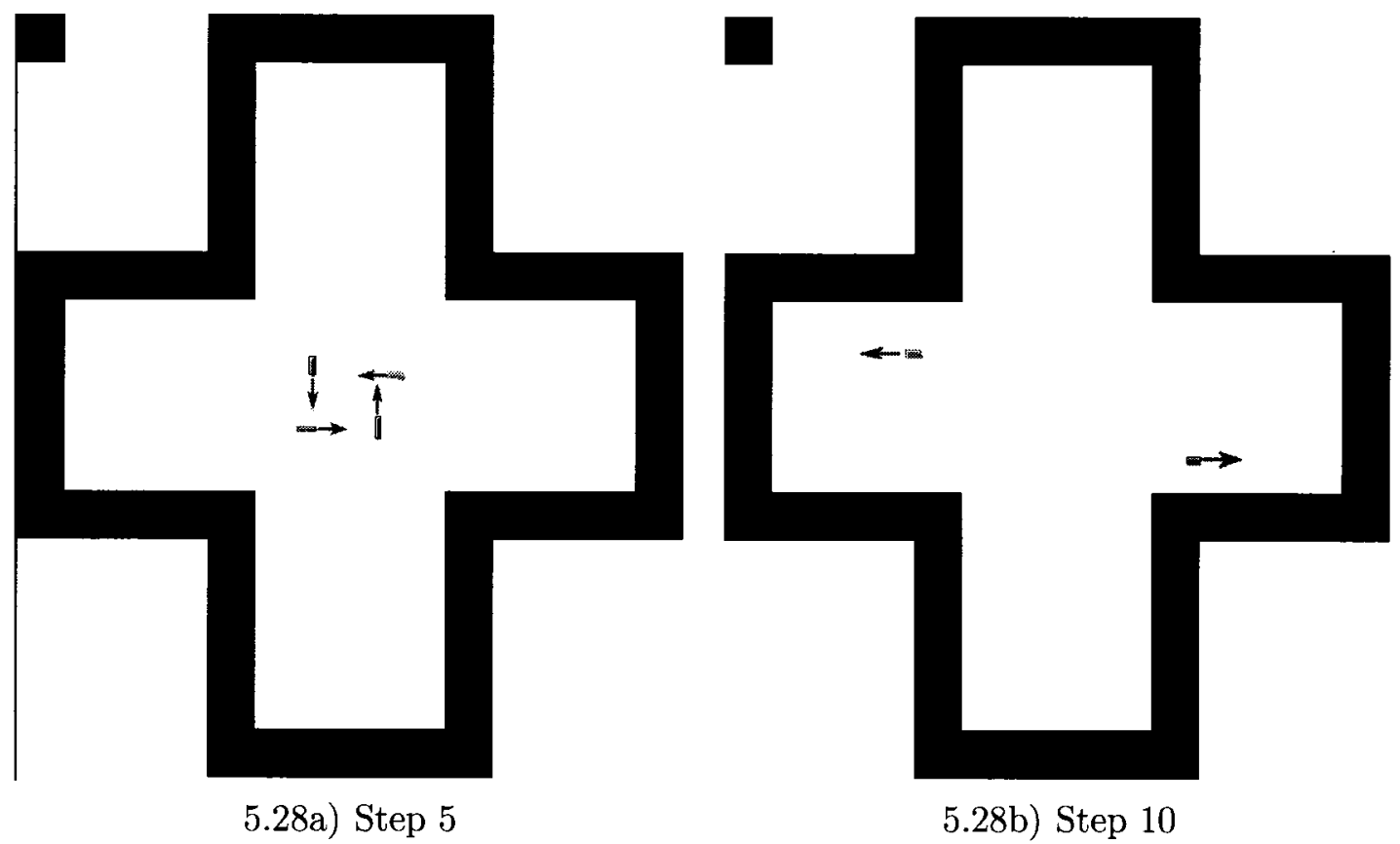

Figure 5.28: Example of CPF-MCL Keeping Symmetric Locations from Test Case $\mathrm{CPF}-1$

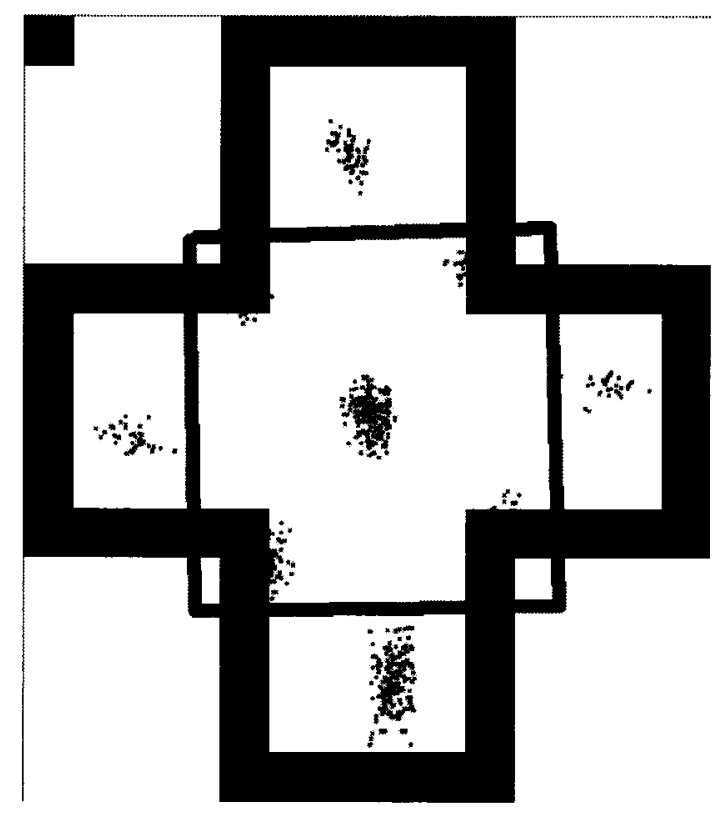

Figure 5.29: Example of U-MCL Failing in a General Environment 
Table 5.3: Summarised MCL1 Symmetric Map Test Case Results

\begin{tabular}{|c||c|c|c|c|c|l|}
\hline \hline Test Case & \# Clusters & \# Reloc & Criterion 1 & Criterion 2 & Criterion 3 & Interpretation \\
\hline MCL1-1 & 1 & 1 & $\mathbf{X}$ & $\checkmark$ & $\mathbf{X}$ & $\begin{array}{l}\text { Vertical reflection } \\
\text { of true location }\end{array}$ \\
\hline MCL1-2 & 1 & 0 & $\checkmark$ & $\mathbf{X}$ & $\mathbf{X}$ & \\
\hline MCL1-3 & 1 & 0 & $\mathbf{X}$ & $\checkmark$ & $\mathbf{X}$ & $\begin{array}{l}\text { Rotate 180'to get } \\
\text { true location }\end{array}$ \\
\hline MCL1-4 & 1 & 0 & $\checkmark$ & $\mathbf{X}$ & $\mathbf{X}$ & \\
\hline MCL1-5 & 1 & 0 & $\checkmark$ & $\mathbf{X}$ & $\mathbf{X}$ & \\
\hline MCL1-6 & 1 & 0 & $\checkmark$ & $\mathbf{X}$ & $\mathbf{X}$ & \\
\hline MCL1-7 & 1 & 0 & $\checkmark$ & $\mathbf{X}$ & $\mathbf{X}$ & \\
\hline MCL1-8 & 1 & 0 & $\checkmark$ & $\mathbf{X}$ & $\mathbf{X}$ & \\
\hline MCL1-9 & 1 & 0 & $\checkmark$ & $\mathbf{X}$ & $\mathbf{X}$ & \\
\hline \hline
\end{tabular}

Table 5.4: Summarised MCL2 Symmetric Map Test Case Results

\begin{tabular}{|c||c|c|c|c|c|l|}
\hline \hline Test Case & \# Clusters & \# Reloc & Criterion 1 & Criterion 2 & Criterion 3 & Interpretation \\
\hline MCL2-1 & 1 & 1 & $\checkmark$ & $\mathbf{X}$ & $\mathbf{X}$ & \\
\hline MCL2-2 & 1 & 0 & $\checkmark$ & $\mathbf{X}$ & $\mathbf{X}$ & \\
\hline MCL2-3 & 1 & 0 & $\checkmark$ & $\mathbf{X}$ & $\mathbf{X}$ & \\
\hline MCL2-4 & 1 & 1 & $\checkmark$ & $\mathbf{X}$ & $\mathbf{X}$ & \\
\hline MCL2-5 & 1 & 0 & $\checkmark$ & $\mathbf{X}$ & $\mathbf{X}$ & \\
\hline MCL2-6 & 1 & 0 & $\checkmark$ & $\mathbf{X}$ & $\mathbf{X}$ & \\
\hline MCL2-7 & 1 & 1 & $\mathbf{X}$ & $\mathbf{X}$ & $\mathbf{X}$ & $\begin{array}{l}\text { Close to being a } \\
\text { vertical reflection }\end{array}$ \\
\hline MCL2-8 & 1 & 1 & $\checkmark$ & $\mathbf{X}$ & $\mathbf{X}$ & \\
\hline MCL2-9 & 1 & 0 & $\checkmark$ & $\mathbf{X}$ & $\mathbf{X}$ & \\
\hline
\end{tabular}

Table 5.7 summarises the results of using the UCPF-MCL method. The following are observed:

- UCPF-MCL is able to distinguish the true location in all test cases.

- UCPF-MCL is able to keep the distinguishable locations in most test cases. The UCPF-MCL never keeps the similar locations it finds at the end of the path taken by the robot. If it finds similar locations, it is quickly eliminated when the robot enters a corridor, and because the UCPF-MCL test cases all 
Table 5.5: Summarised MCL3 Symmetric Map Test Case Results

\begin{tabular}{|c||c|c|c|c|c|l|}
\hline \hline Test Case & \# Clusters & \# Reloc & Criterion 1 & Criterion 2 & Criterion 3 & Interpretation \\
\hline MCL3-1 & 1 & 6 & $\mathbf{X}$ & $\mathbf{X}$ & $\checkmark$ & $\begin{array}{l}\text { Rotate }-90^{\circ} \text { to get } \\
\text { true location }\end{array}$ \\
\hline MCL3-2 & 1 & 1 & $\mathbf{X}$ & $\mathbf{X}$ & $\checkmark$ & $\begin{array}{l}\text { Rotate }-90^{\circ} \text { to get } \\
\text { true location }\end{array}$ \\
\hline MCL3-3 & 1 & 0 & $\mathbf{X}$ & $\mathbf{X}$ & $\checkmark$ & $\begin{array}{l}\text { Rotate }-90^{\circ} \text { to get } \\
\text { true location }\end{array}$ \\
\hline MCL3-4 & 1 & 4 & $\checkmark$ & $\mathbf{X}$ & $\mathbf{X}$ & \\
\hline MCL3-5 & 1 & 1 & $\mathbf{X}$ & $\mathbf{X}$ & $\checkmark$ & $\begin{array}{l}\text { Rotate } 90^{\circ} \text { to get } \\
\text { true location }\end{array}$ \\
\hline MCL3-6 & 1 & 1 & $\mathbf{X}$ & $\mathbf{X}$ & $\checkmark$ & $\begin{array}{l}\text { Rotate }-90^{\circ} \text { to get } \\
\text { true location }\end{array}$ \\
\hline MCL3-7 & 1 & 3 & $\checkmark$ & $\mathbf{X}$ & $\mathbf{X}$ & $\begin{array}{l}\text { Rotate } 90^{\circ} \text { to get } \\
\text { true location }\end{array}$ \\
\hline MCL3-8 & 1 & 0 & $\mathbf{X}$ & $\mathbf{X}$ & $\checkmark$ & $\begin{array}{l}\text { Rotate } 90^{\circ} \text { to get } \\
\text { true location }\end{array}$ \\
\hline MCL3-9 & 1 & 0 & $\mathbf{X}$ & $\mathbf{X}$ & $\checkmark$ \\
\hline \hline
\end{tabular}

Table 5.6: Summarised CPF Symmetric Map Test Case Results

\begin{tabular}{|c||c|c|c|c|c|c|}
\hline \hline Test Case & \# Clusters & \# Reloc & Criterion 1 & Criterion 2 & Criterion 3 & Interpretation \\
\hline CPF-1 & 2 & 0 & $\checkmark$ & $\checkmark$ & $\mathbf{X}$ & \\
\hline CPF-2 & 4 & 0 & $\checkmark$ & $\checkmark$ & $\checkmark(2)$ & \\
\hline CPF-3 & 4 & 0 & $\checkmark$ & $\checkmark$ & $\checkmark(2)$ & \\
\hline CPF-4 & 2 & 0 & $\checkmark$ & $\checkmark$ & $\mathbf{X}$ & \\
\hline CPF-5 & 4 & 0 & $\checkmark$ & $\checkmark$ & $\checkmark(2)$ & \\
\hline CPF-6 & 4 & 0 & $\checkmark$ & $\checkmark$ & $\checkmark(2)$ & \\
\hline CPF-7 & 2 & 0 & $\checkmark$ & $\checkmark$ & $\mathbf{X}$ & \\
\hline CPF-8 & 4 & 0 & $\checkmark$ & $\checkmark$ & $\checkmark(2)$ & \\
\hline CPF-9 & 4 & 0 & $\checkmark$ & $\checkmark$ & $\checkmark(2)$ & \\
\hline \hline
\end{tabular}

ran without need for relocalisation, only the distinguishable similar locations are normally found. In test case UCPF-3 and UCPF-9, only the true location of the robot is found, but that is most likely due to the Monte Carlo sampling process injecting samples at the robot's true location by chance, and none at the other distinguishable similar location. 
Table 5.7: Summarised UCPF Symmetric Map Test Case Results

\begin{tabular}{|c||c|c|c|c|c|c|}
\hline \hline Test Case & \# Clusters & \# Reloc & Criterion 1 & Criterion 2 & Criterion 3 & Interpretation \\
\hline UCPF-1 & 2 & 0 & $\checkmark$ & $\checkmark$ & $\mathbf{X}$ & \\
\hline UCPF-2 & 2 & 0 & $\checkmark$ & $\checkmark$ & $\mathbf{X}$ & \\
\hline UCPF-3 & 1 & 0 & $\checkmark$ & $\mathbf{X}$ & $\mathbf{X}$ & \\
\hline UCPF-4 & 2 & 0 & $\checkmark$ & $\checkmark$ & $\mathbf{X}$ & \\
\hline UCPF-5 & 2 & 0 & $\checkmark$ & $\checkmark$ & $\mathbf{X}$ & \\
\hline UCPF-6 & 2 & 0 & $\checkmark$ & $\checkmark$ & $\mathbf{X}$ & \\
\hline UCPF-7 & 2 & 0 & $\checkmark$ & $\checkmark$ & $\mathbf{X}$ & \\
\hline UCPF-8 & 2 & 0 & $\checkmark$ & $\checkmark$ & $\mathbf{X}$ & \\
\hline UCPF-9 & 1 & 1 & $\checkmark$ & $\mathbf{X}$ & $\mathbf{X}$ & \\
\hline \hline
\end{tabular}

\subsubsection{Office Map Test Cases}

Based on the results of the symmetric map test cases, the localisation methods tested all performed as expected, and the results are excellent. There are little to no relocalisations required, and the methods usually localised the robot. In this section, the localisation methods are tested against a larger office-like environment. Like in the symmetric map test cases, the office map test cases vary parameters. The parameters varied are the number of initial samples, the level of sensor noise, and the level of motion noise. The tests are all run using the office map test environment described in Section 5.1.2. Multiple sets of test cases are executed for the office environment. The first set examines single robot simulations through the environment. Since it is hard to draw any reasonable conclusions with a single execution of different test cases, other sets of test cases are run repeatedly to collect statistics.

\section{Test Set of Single Runs}

The test cases that belong to this test set are listed in Table 5.8. In general, the test cases can be categorised as over estimated noise test cases, and under estimated noise test cases. The set of over estimated noise test cases includes all test cases that have 
a motion noise of $10 \%$. The name of the set refers to the fact that the noise of the robot's motion is only $10 \%$ of its projected ideal motion, while the robot's odometry sensor expects a motion noise of $30 \%$. Conversely, the set of under estimated noise test cases includes all test cases that have a motion noise of $50 \%$.

Table 5.8: Localisation Office Map Test Cases for Varying Parameters

\begin{tabular}{|c|c|c|c|c|c|}
\hline Test Case \# & $\begin{array}{l}\text { Initial } \\
\text { Sample Size }\end{array}$ & $\begin{array}{l}\text { Cluster } \\
\text { Sample Size }\end{array}$ & $\begin{array}{l}\text { Sensor } \\
\text { Noise }(\mathrm{m})\end{array}$ & $\begin{array}{l}\text { Motion } \\
\text { Noise (\%) }\end{array}$ & Results \\
\hline O-MCL-1 & \multirow{6}{*}{500,000} & $\mathrm{~N} / \mathrm{A}$ & \multirow{3}{*}{0.1} & \multirow{3}{*}{ 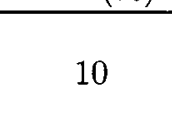 } & Table B-46 \\
\hline O-CPF-1 & & \multirow{2}{*}{1000} & & & Table B-52 \\
\hline O-UCPF-1 & & & & & Table B-58 \\
\hline O-MCL-2 & & $\mathrm{N} / \mathrm{A}$ & \multirow{3}{*}{0.5} & \multirow{3}{*}{50} & Table B-47 \\
\hline O-CPF-2 & & \multirow{2}{*}{1000} & & & Table B-53 \\
\hline O-UCPF-2 & & & & & Table B-59 \\
\hline O-MCL-3 & \multirow{6}{*}{100,000} & $\mathrm{~N} / \mathrm{A}$ & \multirow{3}{*}{0.1} & \multirow{3}{*}{10} & Table B-48 \\
\hline O-CPF-3 & & & & & Table B-54 \\
\hline O-UCPF-3 & & 1000 & & & Table B-60 \\
\hline O-MCL-4 & & $\mathrm{N} / \mathrm{A}$ & \multirow{3}{*}{0.5} & \multirow{3}{*}{50} & Table B-49 \\
\hline O-CPF-4 & & \multirow{2}{*}{1000} & & & Table B-55 \\
\hline O-UCPF-4 & & & & & Table B-61 \\
\hline O-MCL-5 & \multirow{6}{*}{10,000} & $\mathrm{~N} / \mathrm{A}$ & \multirow{3}{*}{0.1} & \multirow{3}{*}{10} & Table B-50 \\
\hline O-CPF-5 & & & & & Table B-56 \\
\hline O-UCPF-5 & & 1000 & & & Table B-62 \\
\hline O-MCL-6 & & $\mathrm{N} / \mathrm{A}$ & \multirow{3}{*}{0.5} & \multirow{3}{*}{50} & Table B-51 \\
\hline O-CPF-6 & & \multirow{2}{*}{1000} & & & Table B-57 \\
\hline O-UCPF-6 & & & & & Table B-63 \\
\hline
\end{tabular}

For this larger office map, the following data are collected from the test cases:

\# Reloc: This datum is the number of relocalisations. A high value for this indicator suggests that the method cannot initially localise the robot, or cannot track the robot as it moves through the environment. This datum does not apply to the CPF-MCL, since it has a background task that constantly adds new possible clusters, and never relocalises from scratch. Relocalisation occurs when all particles in the localisation method are improbable. In the case of MCL, this 
occurs when all particles have zero weight. In the case of UCPF-MCL, this occurs when all clusters are removed from the filter.

\# MLC Success: This datum is the number of successes according to the most likely cluster (MLC). The number of successful localisations, compared to the true value, as the robot moves through the environment is a good measure of whether the method is able to localise the robot. Since the localisation methods may be multimodal (have more than one cluster of particles far apart from each other), the most likely cluster is compared to the true value. The likelihood of the cluster is determined using the weights of the particles in the cluster.

\# CC Success This datum is the number of successes according to the closest cluster (CC). With this datum, the cluster closest to the true value is examined for success. This value can only be computed offline since the robot does not know its true location as it is running. It is possible that the cluster that best localises the robot may not have the highest weight, even though it may have a similarly high weight.

The summarised test results are displayed in Table 5.9. The following observations are made from the results:

Effect of Decreased Number of Initial Samples As can be seen in the results, a decreased number of samples adversely affects most of the test cases, as expected. As seen in Figure 5.30, only the case of CPF with 50\% noise contradicts this trend significantly.

Seen in Table 5.9, the decreased number of samples also tends to increase the number of relocalisations needed in the methods that require relocalisations. 
Effect of Over Estimated and Under Estimated Odometry Noise It should be noted that there are two types of noise that are of concern. The first is the amount of noise simulated for the robot's actual motion as it moves through the environment. The second is the amount of motion noise expected by the robot as part of its localisation routines. In these experiments, the odometry sensor used in the virtual robot expects that its calculated expected odometry is corrupted by a zero mean Gaussian noise process with a standard deviation of $30 \%$ of the expected odometry. The amount of noise simulated for the robot's actual motion is a varied parameter in the test cases. The over estimated odometry test cases corrupt the robot's true motion with a zero mean Gaussian noise process with a standard deviation of $10 \%$ of the ideal distance travelled (ie: O-MCL-1). The under estimated odometry test cases corrupt the robot's true motion with a zero mean Gaussian noise process with a standard deviation of $50 \%$ of the ideal distance travelled. Comparing the over estimated odometry noise test cases to the under estimated odometry noise test cases, it can be seen from Table 5.9 that over estimated odometry noise yields better localisation results than under estimated odometry noise.

\section{Test Set of Repeated Runs}

Due to the randomness of the simulation, the observations from the test set of single runs may not be valid. The test case settings in Table 5.10 are run for each localisation method multiple times to collect various statistics. The tables of results for all the repeated runs are given in Table 5.11, Table 5.12 Table 5.13, Table 5.14, Table 5.15, and Table 5.16. The various statistics are:

MLC The most likely cluster (MLC) statistic is the percentage of times that a single 
Table 5.9: Summarised Office Map Single Run Test Case Results

\begin{tabular}{|c|c|c|c|c|}
\hline Test Case & \# Reloc & \# MLC Success & \# CC Success & Notes \\
\hline O-MCL-1 & 4 & $41 / 45$ & $41 / 45$ & \\
\hline O-MCL-2 & 3 & $23 / 45$ & $24 / 45$ & $\begin{array}{l}\text { Did not work well in symmet- } \\
\text { ric area. Some results not } \\
\text { counted due to being slightly } \\
\text { over threshold }\end{array}$ \\
\hline O-MCL-3 & 9 & $34 / 45$ & $34 / 45$ & $\begin{array}{l}\text { Relocalisations occurred in } \\
\text { symmetric area, and initial } \\
\text { stages }\end{array}$ \\
\hline O-MCL-4 & 3 & $24 / 45$ & $25 / 45$ & $\begin{array}{l}\text { Did not work well in symmetric } \\
\text { area, and initial stages }\end{array}$ \\
\hline O-MCL-5 & 22 & $18 / 45$ & $18 / 45$ & \multirow{2}{*}{$\begin{array}{l}\text { Did not work well in initial } \\
\text { stages. }\end{array}$} \\
\hline O-MCL-6 & 7 & $15 / 45$ & $16 / 45$ & \\
\hline O-CPF-1 & & $38 / 45$ & $42 / 45$ & \multirow{6}{*}{$\begin{array}{l}\text { Tended to work in initial } \\
\text { stages }\end{array}$} \\
\hline O-CPF-2 & & $24 / 45$ & $32 / 45$ & \\
\hline $\mathrm{O}-\mathrm{CPF}-3$ & & $27 / 45$ & $34 / 45$ & \\
\hline O-CPF-4 & & $30 / 45$ & $38 / 45$ & \\
\hline O-CPF-5 & & $13 / 45$ & $15 / 45$ & \\
\hline O-CPF-6 & & $26 / 45$ & $37 / 45$ & \\
\hline O-UCPF-1 & $\overline{2}$ & $41 / 45$ & $42 / 45$ & \\
\hline O-UCPF-2 & 0 & $40 / 45$ & $41 / 45$ & \\
\hline O-UCPF-3 & 4 & $34 / 45$ & $34 / 45$ & $\begin{array}{l}\text { Relocalisations occurred in ini- } \\
\text { tial stages }\end{array}$ \\
\hline O-UCPF-4 & 1 & $41 / 45$ & $41 / 45$ & $\begin{array}{l}\text { Relocalisation occurred in ini- } \\
\text { tial stages }\end{array}$ \\
\hline O-UCPF-5 & 5 & $32 / 45$ & $32 / 45$ & \\
\hline O-UCPF-6 & 10 & $17 / 45$ & $17 / 45$ & $\begin{array}{l}\text { Could not relocalise after los- } \\
\text { ing track in the middle of test } \\
\text { run }\end{array}$ \\
\hline & & & & $\begin{array}{l}\text { ment, it localises approximately } \\
\text { ttempts, and each set of data is } \\
\text { data is within } 1 \mathrm{~m}^{2} \text { of the true }\end{array}$ \\
\hline
\end{tabular}

run successfully localises the robot using the most likely cluster. The most likely cluster is determined according to the weights of the clusters. In the tables of results, the first number given is the mean percentage of successful localisations, and the second number is the standard deviation.

MLS The most likely sample (MLS) statistic is the percentage of times that a single 


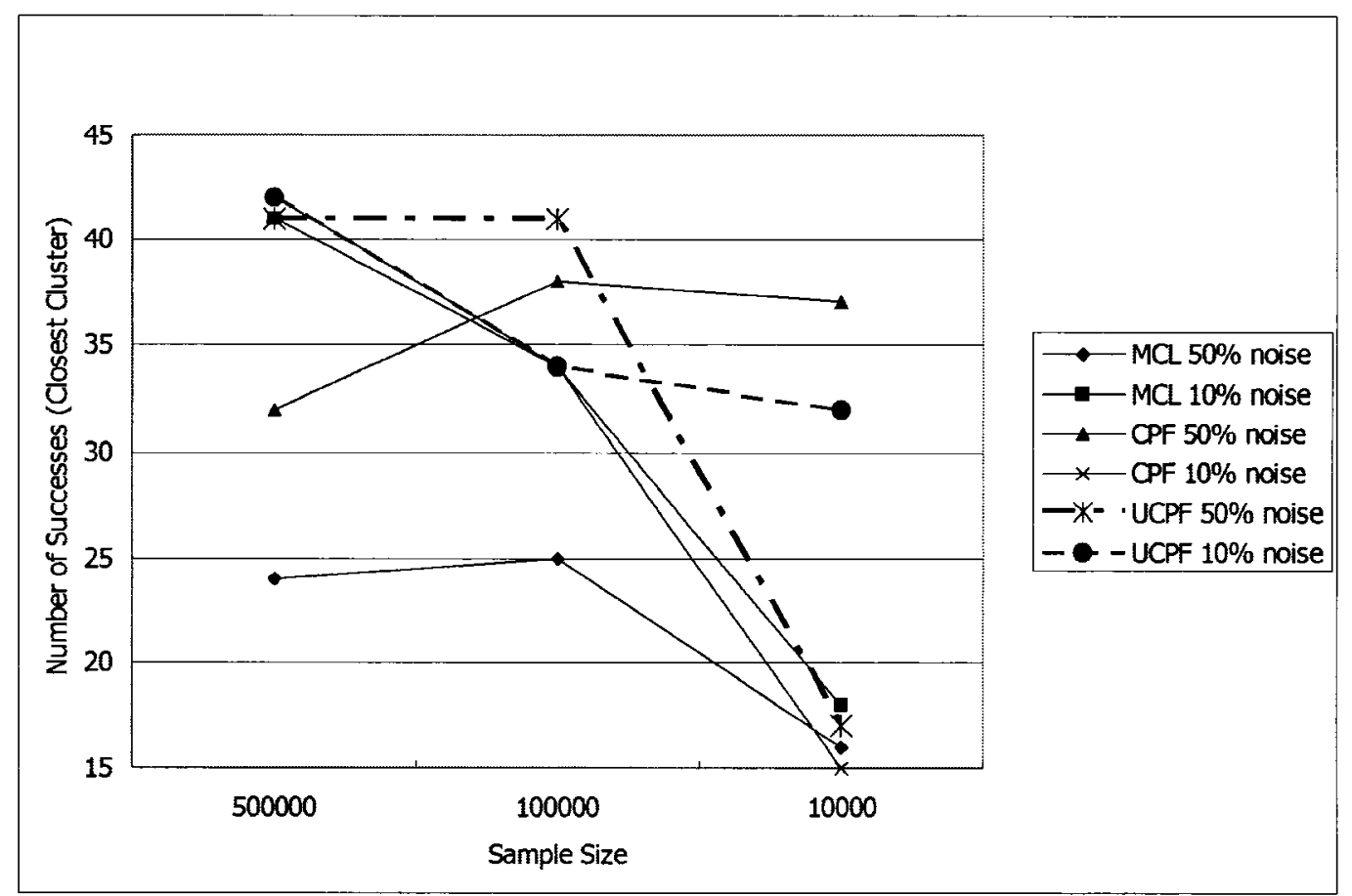

Figure 5.30: Chart Summarising Single Run Success

run successfully localises the robot using the most likely sample according to the weights of all the particles in the particle filter. In the tables of results, the first number is the mean percentage of successful localisations, and the second number is the standard deviation.

CC The closest cluster (CC) statistic is the percentage of times that a single run successfully localises the robot using the closest cluster. The closest cluster may not have the highest weight. In the tables of results, the first number is the mean percentage of successful localisations, and the second is the standard deviation.

STREAK The STREAK statistic is the measure of the maximum number of localisation attempts that occurred without the need for global relocalisation, much 
like a streak of successful localisations. This measure indicates the ability for the localisation method to globally localise the robot and track its motion through the environment. The first number given in the tables is the mean number of successes, while the second number given is the standard deviation.

MINMAX(STREAK) This statistic gives the minimum and maximum number of "lucky" continuous successful localisation attempts.

RELOC This statistic gives the number of global relocalisations needed through the different repeated runs of the test cases. Relocalisations occur when all particles of the localisation methods are regarded as improbable. For the case of MCL, this occurs when all particles are given zero weight. For the case of UCPFMCL, this occurs when all particle clusters are removed from the filter. The first number given in the tables is the mean, while the second is the standard deviation.

SUCC $(\cdot) \operatorname{SUCC}(\cdot)$ gives the number of times the robot is successfully localised after the repeated treks through the environment. A successful localisation is defined to be sufficiently close to the final destination at the end of the test run for a given criterion. Valid criteria to use are MLC, MLS, and CC. In the tables of results, the first number is the number of successful localisations, and the second is the total number of localisation attempts.

The observations made from the set of single run test cases are seen again in this test set of repcated runnings. As the sample size is decreased, the localisation performance of all methods is adversely affected, and the number of relocalisations is increased (Figure 5.31a, Figure 5.31b). Also, it can be seen that over estimated 
Table 5.10: Test Case Settings for Repeated Runs

\begin{tabular}{|c||c|c|c|}
\hline \hline Test Set & Sample Size & Sensor Noise & Motion Noise \\
\hline R1-1 & \multirow{2}{*}{10,000} & $10 \%$ & $10 \%$ \\
\cline { 3 - 4 } R1-2 & & $50 \%$ & $50 \%$ \\
\hline R2-1 & \multirow{2}{*}{100,000} & $10 \%$ & $10 \%$ \\
\cline { 3 - 4 } R2-2 & & $50 \%$ & $50 \%$ \\
\hline R3-1 & \multirow{2}{*}{500,000} & $10 \%$ & $10 \%$ \\
\cline { 3 - 4 } R3-2 & & $50 \%$ & $50 \%$ \\
\hline
\end{tabular}

odometry noise performs better than under estimated odometry noise. More detailed examination is given below for each localisation method.

For MCL, an increased number of samples improves all performance measures in the case where there is over estimated noise. The MLC, MLS, and CC success measures are increased, along with the final success measures SUCC(MLC), SUCC(MLS), and SUCC $(\mathrm{CC})$. This is expected since as the number of samples are increased, the Monte Carlo method has a better representation of the continuous quantities involved. There is more likelihood of finding a sample that represents the robot state well. With a higher likelihood of finding proper samples to represent the robot state, the number of relocalisations required is decreased. The mean STREAK measure does not increase much from R1-1 to R2-1 for the MCL method, but the standard deviation is increased, an indication that the STREAK measure is more spread out than before.

In the case where there is under estimated noise for the MCL method, the same observations are valid. However, under estimated noise causes MCL to have worse performance when compared to over estimated noise.

For CPF-MCL, an increased number of samples improves all performance measures in the case where there is over estimated noise and under estimated noise, and all the observations made for MCL are equally valid except where the measure is not applicable. Such measures are MLS, STREAK, MINMAX(STREAK), and RE- 


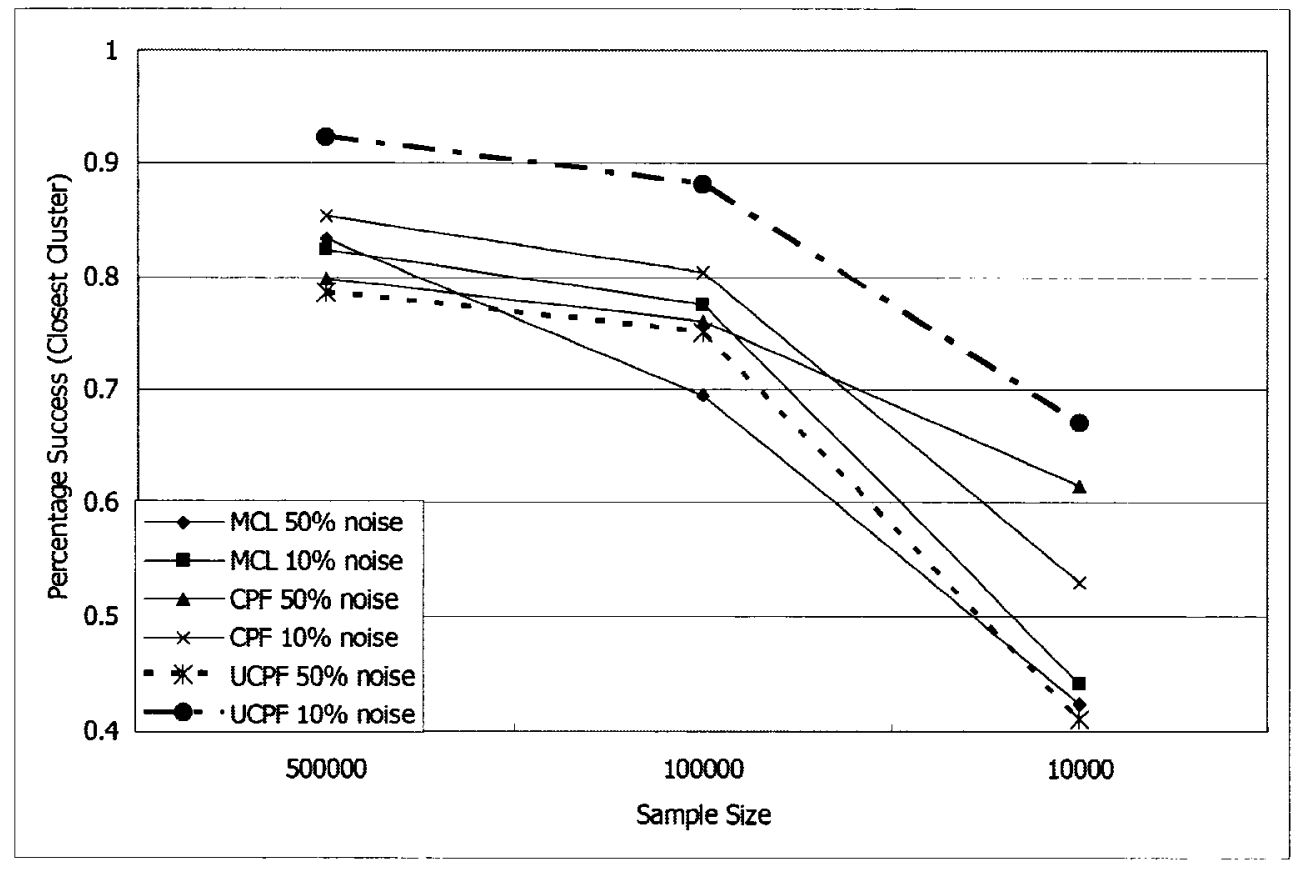

5.31a) Effect of Sample Size on Success of Repeated Runs

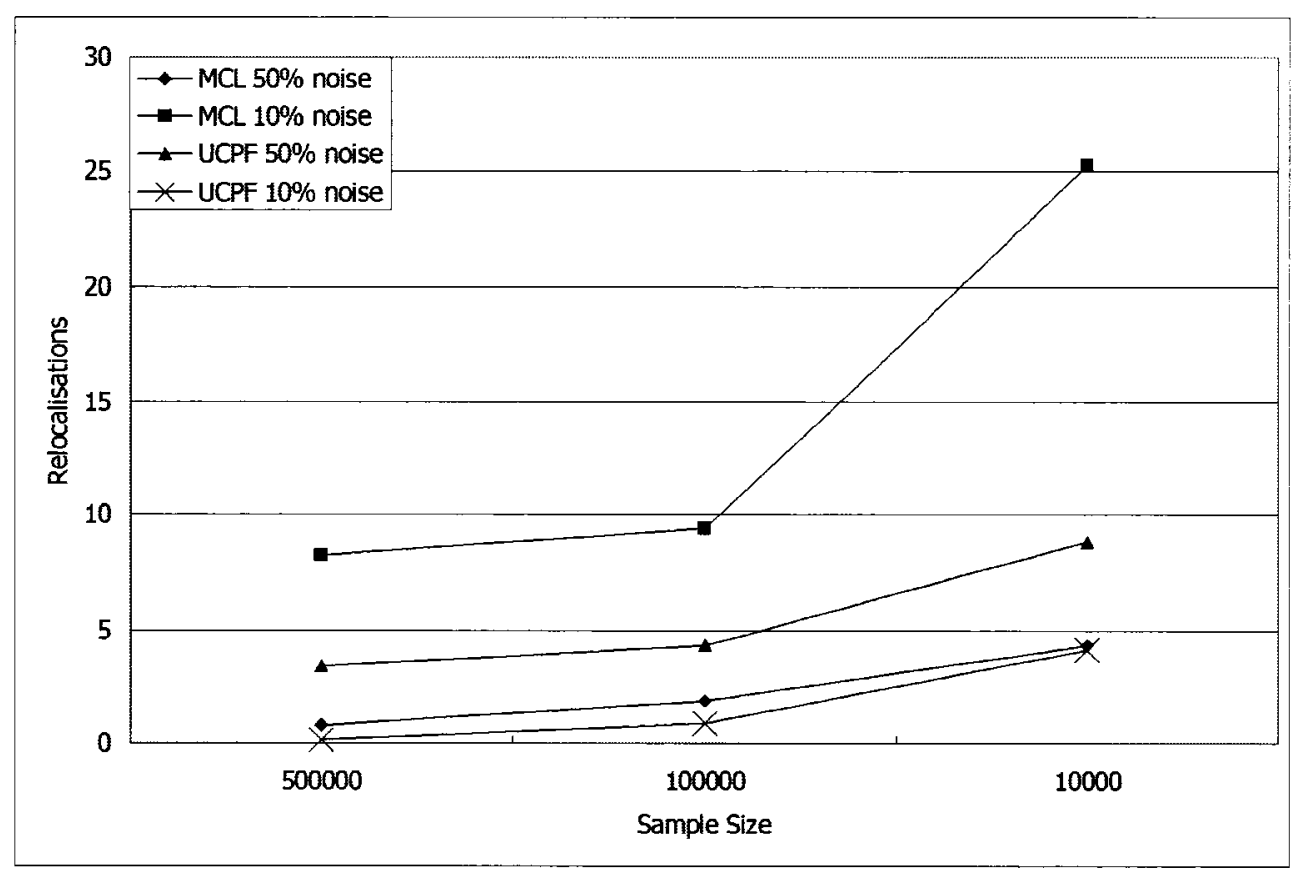

5.31b) Effect of Sample Size on Relocalisation

Figure 5.31: Summary Charts for Repeated Runs Test Cases 
LOC. One observation can be made specific to the CPF-MCL method, in that the MLC measure typically performs worse than the CC measure. This indicates that the CPF-MCL method normally selects the incorrect cluster to represent the most likely location of the robot, when in fact, a better cluster exists. Some ideas for improvement of the MLC measure are detailed in Section 6.1.3.

All of the observations made for MCL are valid for the UCPF-MCL method as well, except for the MLS related measures which are not applicable.

Table 5.11: Summarised Office Map Test Case R1-1 Results

\begin{tabular}{|c||c|c|c|}
\hline \hline & MCL & CPFMCL & UCPFMCL \\
\hline MLC & $0.4326 / 0.2079$ & $0.3223 / 0.0975$ & $0.6623 / 0.1648$ \\
\hline MLS & $0.4332 / 0.2083$ & & \\
\hline CC & $0.4422 / 0.2093$ & $0.5306 / 0.1491$ & $0.6707 / 0.1644$ \\
\hline STREAK & $20.56 / 1.8753$ & & $45.98 / 61.8502$ \\
\hline MINMAX(STREAK) & $20 / 33$ & & $21 / 224$ \\
\hline RELOC & $25.28 / 8.6166$ & & $4.1 / 2.9085$ \\
\hline SUCC (MLC) & $36 / 50$ & $6 / 50$ & $46 / 50$ \\
\hline SUCC (MLS) & $36 / 50$ & & \\
\hline SUCC (CC) & $36 / 50$ & $37 / 50$ & $47 / 50$ \\
\hline
\end{tabular}

Table 5.12: Summarised Office Map Test Case R1-2 Results

\begin{tabular}{|c||c|c|c|}
\hline \hline & MCL & CPFMCL & UCPFMCL \\
\hline MLC & $0.3997 / 0.2624$ & $0.3709 / 0.1172$ & $0.4005 / 0.2322$ \\
\hline MLS & $0.3999 / 0.263$ & & \\
\hline CC & $0.424 / 0.2649$ & $0.6152 / 0.1656$ & $0.4102 / 0.2338$ \\
\hline STREAK & $41.68 / 35.8383$ & & $25.84 / 6.2151$ \\
\hline MINMAX(STREAK) & $22 / 224$ & & $21 / 36$ \\
\hline RELOC & $4.34 / 2.2822$ & & $8.82 / 4.2508$ \\
\hline SUCC (MLC) & $37 / 50$ & $13 / 50$ & $28 / 50$ \\
\hline SUCC (MLS) & $36 / 50$ & & \\
\hline SUCC (CC) & $39 / 50$ & $46 / 50$ & $29 / 50$ \\
\hline
\end{tabular}


Table 5.13: Summarised Office Map Test Case R2-1 Results

\begin{tabular}{|c||c|c|c|}
\hline \hline & MCL & CPFMCL & UCPFMCL \\
\hline MLC & $0.7509 / 0.1017$ & $0.4326 / 0.0495$ & $0.8711 / 0.0607$ \\
\hline MLS & $0.7526 / 0.101$ & & \\
\hline CC & $0.7754 / 0.0949$ & $0.8038 / 0.0606$ & $0.8822 / 0.0601$ \\
\hline STREAK & $22.7 / 4.2246$ & & $122.76 / 101.6682$ \\
\hline MINMAX(STREAK) & $20 / 33$ & & $21 / 225$ \\
\hline RELOC & $9.44 / 4.3479$ & & $0.88 / 1.1183$ \\
\hline SUCC (MLC) & $48 / 50$ & $5 / 50$ & $48 / 50$ \\
\hline SUCC (MLS) & $48 / 50$ & & \\
\hline SUCC (CC) & $48 / 50$ & $48 / 50$ & $49 / 50$ \\
\hline
\end{tabular}

Table 5.14: Summarised Office Map Test Case R2-2 Results

\begin{tabular}{|c||c|c|c|}
\hline \hline & MCL & CPFMCL & UCPFMCL \\
\hline MLC & $0.6624 / 0.1626$ & $0.4438 / 0.067$ & $0.7196 / 0.1088$ \\
\hline MLS & $0.6641 / 0.1616$ & & \\
\hline CC & $0.6959 / 0.1651$ & $0.7606 / 0.1004$ & $0.7511 / 0.0956$ \\
\hline STREAK & $67.4 / 64.0637$ & & $46.38 / 63.6617$ \\
\hline MINMAX(STREAK) & $25 / 225$ & & $21 / 223$ \\
\hline RELOC & $1.88 / 1.1364$ & & $4.34 / 2.5202$ \\
\hline SUCC (MLC) & $49 / 50$ & $5 / 50$ & $34 / 50$ \\
\hline SUCC (MLS) & $49 / 50$ & & \\
\hline SUCC (CC) & $49 / 50$ & $48 / 50$ & $41 / 50$ \\
\hline
\end{tabular}

\subsubsection{Discussion}

Environment similarity has detrimental effects to the localisation performance of the different methods. As seen in Section 5.3.1, the standard MCL method does not deal

Table 5.15: Summarised Office Map Test Case R3-1 Results

\begin{tabular}{|c||c|c|c|}
\hline \hline & MCL & CPF-MCL & UCPF-MCL \\
\hline MLC & $0.7824 / 0.1573$ & $0.422 / 0.0869$ & $0.9063 / 0.0288$ \\
\hline MLS & $0.7868 / 0.1586$ & & \\
\hline CC & $0.8235 / 0.1381$ & $0.8531 / 0.1598$ & $0.9236 / 0.028$ \\
\hline STREAK & $28.05 / 16.311$ & & $206.86 / 55.0477$ \\
\hline MINMAX(STREAK) & $20 / 95$ & & $22 / 226$ \\
\hline RELOC & $8.2 / 7.7599$ & & $0.12 / 0.3854$ \\
\hline SUCC (MLC) & $35 / 40$ & $1 / 42$ & $49 / 50$ \\
\hline SUCC (MLS) & $35 / 40$ & & \\
\hline SUCC (CC) & $35 / 40$ & $40 / 42$ & $50 / 50$ \\
\hline
\end{tabular}


Table 5.16: Summarised Office Map Test Case R3-2 Results

\begin{tabular}{|c||c|c|c|}
\hline \hline & MCL & CPF-MCL & UCPF-MCL \\
\hline MLC & $0.7888 / 0.0983$ & $0.4495 / 0.0577$ & $0.744 / 0.1516$ \\
\hline MLS & $0.8007 / 0.0956$ & & \\
\hline CC & $0.834 / 0.098$ & $0.7986 / 0.0836$ & $0.7864 / 0.152$ \\
\hline STREAK & $120.96 / 80.9789$ & & $70.76 / 72.2056$ \\
\hline MINMAX(STREAK) & $24 / 225$ & & $21 / 224$ \\
\hline RELOC & $0.78 / 0.6158$ & & $3.36 / 2.3713$ \\
\hline SUCC (MLC) & $50 / 50$ & $3 / 50$ & $35 / 50$ \\
\hline SUCC (MLS) & $50 / 50$ & & \\
\hline SUCC (CC) & $50 / 50$ & $49 / 50$ & $43 / 50$ \\
\hline
\end{tabular}

well with similarities in the environment. The MCL method fails to maintain multiple clusters even though there is no distinctive environment feature that distinguishes the multiple clusters. The CPF-MCL method deals with similar environments well, and is able to find clusters that represent the robot's state well, based on sensor observations. In many cases, it is able to find all of the possible clusters that can be the robot's state. The CPF-MCL method, however, is unable to deal with similar locations that are distinguishable due to the differences between the similar corridors of the environment. Though the corridors are similar, they are not identical. This effect is due to its reliance on a background task that constantly scans for new possible clusters. When the robot moves near the spots where only the 2 symmetrical similar clusters can exist, it deletes the other 2 similar clusters that can no longer exist. However, when the robot moves back to the center of the map where the other 2 similar clusters can exist, the background task has a chance of re-inserting the similar clusters that cannot exist when past robot observations and actions are considered. UCPF-MCL, does not suffer from this problem because it does not have a background task. It relocalises only when the robot is completely lost. In this manner, it is the most accurate method in the asymmetrical environment by locating the two correct clusters in most of the test cases. 
Moving to the more general office-like environment in Section 5.3.2, the performance of the localisation methods is again examined. In general, an increased number of samples used in the particle filters presents an increase in localisation performance. This is expected since an increased number of samples allows better representation of continuous probability distributions using Monte Carlo sampling. With increased sampling, the likelihood of proposing samples that correctly represent the true state of the robot is increased, leading to a higher success rate. In the case of UCPF-MCL, for example, the filter will update that incorrect cluster of samples until the robot observes something distinctive about the environment that invalidates that cluster. Only then, will the filter globally relocalise. Looking at the office-like environment, the initial starting location of the robot is a square room with many such similar square rooms throughout the environment. This tends to initially mislead the localisation methods.

A quick look at the results yielded by the methods insinuates that both the CPFMCL and the UCPF-MCL performs better than MCL. The success rate according to the CC measure is typically higher for CPF-MCL in under estimated noise test cases, while the rate is typically higher for UCPF-MCL in over estimated noise test cases under similar test conditions. Though CPF-MCL performs better than UCPF-MCL on average in the under estimated noise test cases, the performance of UCPF-MCL is not much worse. Also, although CPF-MCL performed better in those test cases, it requires the use of the CC measure. CPF-MCL's success rate with the MLC measure, on the other hand, performs poorly. This effect insinuates that the algorithm does not perform well online. Good success rate with the $\mathrm{CC}$ measure indicates that the localisation method proposes a cluster of particles that approximates the robot's true location well, but the method only realises which cluster is the best cluster by 
comparing all clusters to the true location. The cluster that the CPF-MCL thinks is most likely to represent the robot's true location, however, does not represent the robot's location well, as indicated by the poor success rate according to the MLC measure. Ideas on why this is so, and suggestions on improvement is detailed in Section 6.1.3. On the other hand, the performance of both the MCL and the UCPFMCL method according to the MLC measure remains quite close to the CC measure.

An examination of why CPF-MCL beats UCPF-MCL in terms of CC success rate in the test cases with under estimated noise is in order. UCPF-MCL uses a different odometry action model than CPF-MCL, or MCL. UCPF-MCL makes use of a uniform odometry sensor model, as opposed to a Gaussian model. The uniform model assumes that the robot can move any distance between 0 and the projected ideal distance travelled. The actual Gaussian movement model projects that the movement the robot might go through is its ideal distance travelled, with added zero mean Gaussian noise. This means that the actual distance a robot travels might be larger than its ideal movement, whereas the uniform model does not expect that the robot will travel further than its projected ideal distance. A more detailed examination is given in Section 6.1.4, along with suggested improvements that can increase the performance of UCPF-MCL such that it may beat CPF-MCL in all cases.

The STREAK measure is particularly interesting for UCPF-MCL. The measure is higher in over estimated noise test cases of equal sample size, but lower in under estimated noise test cases. Even though this is the case, the success rate according to MLC and CC measures are still competitively high when compared to similar measures for MCL and UCPF-MCL. This indicates that UCPF-MCL is quick to converge to a good solution after global relocalisation, since it does not have long running streaks of success, but maintains a good success rate. The increased use 
of global relocalisation is also apparant with the increase in the RELOC measure when comparing over estimated noise test cases to under estimated noise test cases. This phenomenon is also explainable by the mismatch in odometry action model. Since the uniform odometry action model is unable to propose samples based on the fact the actual distance travelled by the robot may be further than its projected ideal movement. As such, clusters that represent the robot's state well before its movement action no longer represent the robot's state well after its movement, tend to die off, and global relocalisation is required.

A qualitative examination of computational complexity indicates that UCPFMCL is the least complex method, followed by CPF-MCL, and lastly, MCL. For UCPF-MCL, and CPF-MCL, the initial sample size may be set to a large value to quickly globally localise the robot, but after clustering the samples, the particle filters only need a small number of samples for each cluster. MCL, however, retains the same number of particles while the robot traverses the environment. Further contrasting UCPF-MCL and CPF-MCL, UCPF-MCL filters make use of U-MCL filters for each cluster, as opposed to the MCL filter. U-MCL filters are comparatively less computationally complex since it employs much simpler action and sensor models based on the uniform distribution, as well as a simpler resampling process.

All cases considered, the UCPF-MCL method performs better than the other localisation methods examined in this thesis. It has consistently equal, or higher success rate than the other methods using the same measure, is less computationally intensive, and is easy to implement. 


\subsection{Chapter Summary}

Test cases designed to produce results for discussion are described in this chapter for both mapping and localisation methods.

The mapping tests are intended to examine the validity of using the proposed laser sensor model for evidential mapping, and the effects of varying the parameters for the laser sensor model. Namely, the parameters are of the level of support for occupancy, and the level of support for emptiness. The results immediately show the validity of using the proposed laser sensor model, and that all maps created using varying levels of support are all valid. The boundary between empty and occupied areas of the map, however, are mapped differently depending on the varying levels of support. If the level of occupancy support is higher than the emptiness support, then cells that are supposed to be empty near the boundary tend to be mistakenly marked as occupied. The converse is true when the level of emptiness support is higher than the occupancy support. Maps that err on the side of occupancy are more useful for mobile robotics, so that paths can be planned without encountering unexpected collisions. It is also observed for high noise levels that emptiness support tends to bleed into areas that are supposed to be occupied. Ordinarily, such mistakenly given support to a cell is fixed when more sensor observations arrive. Due to the point based nature of the laser sensor model, however, and the fact that the cells mistakenly labeled are far away from the boundary, further sensor observations do not arrive to correct the cell state. It is therefore better to use the occupancy plausibility map as the map to err on the side of caution.

The localisation tests are intended to compare the performance of the different implemented localisation techniques. Similar locations and distinguishable similar 
locations are the key issues compared. The standard MCL method does not deal with similar locations well, while the CPF-MCL is able to find all similar locations. The fact that CPF-MCL is able to find all similar locations, however, is not ideal. Some of the similar locations can be eliminated when taking past sensor observations and actions into account. UCPF-MCL is able to find all similar locations that can exist even when taking past sensor observations and actions into account.

The success rates of the methods are also compared. All methods localised the robot relatively well, but CPF-MCL shows a marked improvement over MCL, while UCPF-MCL gives consistently equal or higher success rates than the other methods. CPF-MCL is shown to perform well offline, but badly online due to its constant addition of new sample clusters. Adjustments to the weighting system for clusters is likely to improve its online performance.

The UCPF-MCL tends to keep the robot localised without the need for global relocalisation longer than the other methods, as implied by the STREAK measurement. Therefore, the UCPF-MCL is better at tracking the robot than the other methods. Another observed strength of UCPF-MCL is that in some test cases, even though the number of relocalisations increases, and is much higher than the other methods in some cases, UCPF-MCL still maintains an equally high, or higher success rate compared to the other methods. This indicates that the UCPF-MCL is possibly better at global relocalisation than the other methods.

Considering the qualitative complexity of the implemented methods, UCPF-MCL comes out on top as the best method, in terms of both ease of implementation, and computational requirements. All things considered, UCPF-MCL is a good method for robot localisation. 


\section{Chapter 6}

\section{Conclusions and Future Research}

Mapping and localisation are introduced to be fundamental issues in Chapter 1. These issues are to be solved for truly autonomous mobile robots. These two problems are intrinsically linked. Mapping without solving the localisation issue creates inaccurate maps. Localisation without a map is an exercise in failure. This thesis considered these two fundamental problems individually, each with their own solutions. Different approaches to mapping and localisation are surveyed in Chapter 2.

Mapping with a grid based map is advantageous in that free space is explicitly modeled. With such a model, collision free path planning is easy and simple to achieve, and is readily usable with popular localisation methods. Furthermore, a topological model can be derived from the grid based map, and present high level interpretations of the environment, and high level path planning. If a topological map was chosen, the converse is not true.

Grid based mapping requires methods to integrate the robot sensors' observations over time. These observations are many, and possibly conflicting; issues that the integration method needs to deal with. Two such methods are considered in Sec- 
tion 3.1: the Bayesian method, and the evidential method. The evidential method is considered to be a more general method that encompasses the Bayesian method, with extra mechanisms to represent uncertainty and the unknown. The evidential method is subsequently implemented in the simulator for a robot equipped with laser range sensors, and tested with different parameters. It is shown by the results in Section 5.2 that varying parameters does not significantly change the map created by the robot, but it does affect the occupancy of cells near occupancy boundaries where free space meets occupied space. Those boundary cells might be misclassified depending on mapping parameters, but it is better for the mapping method to err on the side of occupancy so that paths created using the map are ensured to be collision free. Due to the point based nature of sensor observations from a laser range sensor, there are cases where free space "bleeds" into the occupied space due to noisy measurements. The mapping method cannot correct the misclassified cells that are outside of the boundary region because no further sensor measurements arrive that measure the same spot. Therefore, it is better to use the occupancy plausibility map created by the evidential method to err on the side of occupancy once again. The evidential method is shown to be a viable mapping method for mobile robots that takes uncertain sensor measurements into account.

The localisation problem has many different solutions, but the most popular are based on Bayesian theory. The Monte Carlo localisation (MCL) methods are most popular, and are considered as the de facto standard in present times. MCL methods make use of particle filters to solve the localisation problem, and present the advantages of representing arbitrary probability density functions describing the probability of robot location. The theory behind MCL methods is examined in Section 3.2, and various methods are implemented in the simulator and examined. The standard MCL 
method using a weighted bootstrap filter is implemented, along with the Clustered Particle Filtering MCL (CPF-MCL), Uniform MCL (U-MCL), and Uniform CPFMCL (UCPF-MCL) variants. Results in Section 5.3.1 show that MCL does not deal well with similar locations, while both the CPF-MCL and UCPF-MCL methods perform well in such environments. Under further consideration, the UCPF-MCL method performs best since it can eliminate certain similar locations that are distinguishable given robot observations and actions.

The localisation methods are given more scrutiny in Section 5.3.2. Two test sets are performed, and data collected. The first test set involves single runnings of some test cases. The second test set involves repeated runnings of some test cases with all localisation methods. It can be seen that an increased number of samples improves the performance of all methods, and although the methods still work reasonably well with under estimated noise test cases, they work better with over estimated noise test cases. Analysis indicates that the localisation methods works best when the noise level is not under estimated. UCPF-MCL tends to yield better performance in all respects of success rate, length of run time before global relocalisation is needed, and computational complexity.

\subsection{Further Work}

Through the course of the thesis work, the issues discovered and described here are considered to be most important. 


\subsubsection{Evidential Mapping Discussion}

As the results in this thesis indicate, evidential mapping is a viable mapping method. Its two primary weaknesses are that some maps created under large amounts of noise are useless for the robot, and that the mapping method does not deal with motion noise.

As discussed in Section 5.2.3, some maps created under large amounts of sensor noise cannot be used by the robot. Paths planned with such maps may cause the robot to collide with objects due to the fact some cells in such maps may be mislabeled as being empty. There may be potential to insert a post-processing step to correct mislabeled cells in the map based on the state of surrounding cells, or some other heuristics.

The greatest weakness in evidential mapping is that it does not deal well with motion error. Motion error introduces cumulative error that causes the created map to be erroneous. This is introduced in Chapter 2 as a problem that accentuates the intrinsic relationship between mapping and localisation. It would be interesting if there are any possible solutions that do not couple localisation and mapping together.

\subsubsection{Discussion}

Although the MCL method underperforms when compared to the other methods examined, it is still a relatively good performer, and is simpler in that there is no need for a clustering stage. The biggest flaw in the standard MCL method is the need for a large number of initial samples to present good global relocalisation characteristics. Since the MCL method does not allow online changing of sample size, the large number of samples is used throughout the operation of the robot. Once the robot is 
sufficiently localised, however, the large number of samples used is unnecessary. Many samples will be similar to each other. It would be advantageous to reduce the number of samples when the robot is well localised for decreased computational complexity. Adaptive Sampling MCL (AS-MCL) [44] is a variant that deals with this idea, and it would be interesting to compare its performance with the other methods.

\subsubsection{CPF-MCL Discussion and Improvements}

The results in Section 5.3.2 indicate that the most likely cluster (MLC) of the CPFMCL filter does not produce good success rates, while there usually exists a cluster of the CPF-MCL filter that produces good success rates, called the closest cluster (CC). Calculating the success rate using the CC criterion, however, is impossible for a robot, since the robot does not know its true location in the environment. It is due to the background task that constantly adds new likely clusters of samples into the filter that causes this problem. Consider the case where a cluster represents the true location of the robot quite well, and is propogated through many localisation attempts. If the background task finds a new cluster that is similar to the true location, it will add the new cluster to the filter. There is no gurantee that the new cluster is close to the true location. This new cluster, however, may receive a higher weight than the old cluster because it is more likely according to the current sensor observations. After a few more localisation attempts, however, the newly inserted cluster is removed because it no longer represents the location of the robot well after some motion. The CPF-MCL as it is implemented does not account for past observations and actions of the robot. A "seniority" system, should be added to the CPF-MCL such that the age of a cluster has an effect on the weight of the cluster. Doing so increases the chance of having the most likely cluster being the closest cluster, therefore improving the performance 
of the MLC success rate.

\subsubsection{UCPF-MCL Discussion and Improvements}

The UCPF-MCL method is by far the best performer overall when various issues are considered. The results in Section 5.3.2 indicate that it has consistently higher success rates according to all measures, has fast convergence for global relocalisation, and is less complex than the other methods. The performance can be boosted if the odometry sensor model employed by the robot matches the simulation odometry sensor model better. The odometry sensor model employed by UCPF-MCL uses a uniform distribution that assumes the robot does not travel further than its odometry sensor reading. This is not a bad assumption, since a real odometry measurement usually over estimates the actual motion of the robot due to wheel slip and collisions. The simulator, however, is implemented such that a zero mean Gaussian noise process of variable variance is added to the ideal projected movement distance. This implies that there is possibility of the actual movement being greater than the projected ideal distance, and in such a case, the odometry sensor model employed by UCPF-MCL does not suggest any samples in the correct area, and needs to relocalise. Eliminating this mismatch will yield better results.

\subsection{Future Research}

This thesis investigated an evidential mapping method, and variants of the MCL method to solve the mapping and localisation problems. Each type of method is a good solution to their respective problems. As mentioned earlier, the mapping and localisation problems are closely linked, leading to the formulation of the simultane- 
ous localisation and mapping (SLAM) problem. Traditional solutions to the SLAM problem are based on a dynamic landmark approach using the Kalman filter, and the mapping and localisation problems are solved as a single problem. It is hypothesized that this needs not be the case. Each problem may be solved individually, and combined together to arrive at a different type of SLAM solution. The focus of future research is a new solution to the SLAM problem that combines evidential mapping, and MCL, each of which are good solutions to their own respective problems. 


\section{References}

[1] H. Akashi and H. Kumamoto, "Random sampling approach to state estimation in switching environments," Automatica, vol. 13, pp. 429-434, 1975.

[2] A. Arleo, J. R. Millan, and D. Floreano, "Efficient learning of variable-resolution cognitive maps for autonomous indoor navigation," IEEE Trans. on Robotics and Automation, vol. 15, no. 6, pp. 990-1000, 1999.

[3] D. Austin and P. Jensfelt, "Using multiple Gaussian hypotheses to represent probability distributions for mobile robot localization," in Proc. of the IEEE Int. Conf. on Robotics \& Automation (ICRA00), vol. 2, 2000, pp. 1036-1041.

[4] D. Avots, E. Lim, R. Thibaux, and S. Thrun, "A probabilistic technique for simultaneous localization and door state estimation with mobile robots in dynamic environments," in Proc. of the IEEE Int. Conf. on Intelligent Robots and System, vol. 1, 2002, pp. 521-526.

[5] N. Ayache and O. D. Faugeras, "Building, registrating and fusing noisy visual maps," in Int. Journal Robot. Res., vol. 7, 1988, pp. 804-819.

[6] A. Bandera, C. Urdiales, and F. Sandoval, "An hierarchical approach to gridbased and topological maps integration for autonomous indoor navigation," in Proc of Int. Conf. on Intelligent Robots and Systems, vol. 2, 2001, pp. 883-888.

[7] L. E. Baum, "An inequality and associated maximization technique occuring in 
the statistical analysis of probabilistic functions of Markov chains," Inequalities, vol. 3, pp. 1-8, 1972.

[8] F. Blais, "A review of 20 years of range sensor development," in Videometrics VII, Proceedings of SPIE-IST Electronic Imaging, vol. SPIE 5013, 2003, pp. $62-76$.

[9] J. Borenstein and Y. Koren, "Obstacle avoidance with ultrasonic sensors," IEEE Journal of Robotics and Automation, vol. 4, no. 2, pp. 213-218, 1988.

[10] — - "The vector field histogram - fast obstacle avoidance for mobile robots," IEEE Journal of Robotics and Automation, pp. 278-288, June 1991.

[11] R. A. Brooks, "Elephants don't play chess," Autonomous Robots, vol. 6, pp. $3-15,1990$.

[12] — - "Intelligence without reason," in Proc. of the Int. Joint Conf. on Artificial Intelligence, 1991, pp. 569-595.

[13] - Cambrian Intelligence: the early history of the new AI. Cambridge, MA: MIT Press, 1999.

[14] J. Buhmann, W. Burgard, A. B. Cremers, D. Fox, T. Hofmann, F. Schneider, J. Strikos, and S. Thrun, "The mobile robot rhino," AI Magazine, vol. 16, no. 1, 1995.

[15] W. Burgard, A. B. Cremers, D. Fox, D. Hahnel, G. Lakemeyer, D. Schulz, W. Steiner, and S. Thrun, "Experiences with an interactive museum tour-guide robot," Artificial Intelligence, vol. 114, no. 1-2, pp. 3-55, 1999.

[16] W. Burgard, A. Derr, D. Fox, and A. B. Cremers, "Integrating global position estimation and position tracking for mobile robots: The dynamic Markov localization approach," in Proc. of the IEEE/RSJ Int. Conf. on Intelligent Robots and Systems (IROS'98), 1998. 
[17] W. Burgard, D. Fox, D. Hennig, and T. Schmidt, "Estimating the absolute position of a mobile robot using position probability grids," in Proc. of the Fourteenth National Conf. on Artificial Intelligence (AAAI-96), 1996, pp. 896901.

[18] W. Burgard, D. Fox, H. Jans, C. Matenar, and S. Thrun, "Sonar-based mapping of large-scale mobile robot environments using EM," in Proc. of the Int. Conf. on Machine Learning, 1999.

[19] B. P. Carlin, N. G. Polson, and D. S. Stoffer, "A Monte Carlo approach to nonnormal and nonlinear state-space modeling," Journal of the American Statistical Association, vol. 87, no. 418, pp. 493-500, 1992. [Online]. Available: citeseer.ist.psu.edu/carlin92monte.html

[20] J. Carpenter, P. Clifford, and P. Fearnhead, "Improved particle filter for nonlinear problems," in Proc. of IEE Radar, Sonar and Navigation, vol. 146, 1999, pp. 2-7.

[21] A. R. Cassandra, L. P. Kaelbling, and J. A. Kurien, "Acting under uncertainty: Discrete Bayesian models for mobile robot navigation," in Proceedings of IEEE/RSJ International Conference on Intelligent Robots and Systems, 1996. [Online]. Available: citeseer.ist.psu.edu/cassandra96acting.html

[22] J. A. Castellanos, J. M. M. Montiel, J. Neira, and J. D. Tardos, "The SPmap: A probabilistic framework for simultaneous localization and map building," IEEE Trans. on Robotics and Automation, vol. 15, no. 5, pp. 948-953, 1999.

[23] J. A. Castellanos and J. D. Tardos, Mobile Robot Localization and Map Building: A Multisensor Fusion Approach. Boston, MA: Kluwer Academic Publishers, 2000.

[24] R. H. T. Chan, P. K. S. Tam, and D. N. K. Leung, "Robot navigation in 
unknown terrains via multi-resolution grid maps," in Proc. of Industrial Electronics, Control and Instrumentation, vol. 2, 1991, pp. 1138-1143.

[25] R. Chatila and J. P. Laumond, "Position referencing and consistent world modeling for mobile robots," in Proc. of the 1985 IEEE Int. Conf. on Robotics and Automation, 1985.

[26] P. Cheeseman, R. Smith, and M. Self, "A stochastic map for uncertain spatial relationships," in Fourth Int. Symposium on Robotic Research. MIT Press, 1987.

[27] D. Chen, R. Szczerba, R. Uhran, and J. Uhran, "A framed-quadtree approach for determining Euclidean shortest paths in 2-D environment," IEEE Trans. on Robotics and Automation, vol. 13, no. 5, pp. 668-680, 1997.

[28] R. Chen and J. S. Liu, "Predictive updating methods with application to Bayesian classification," Journal of the Royal Statistical Society, vol. 58, pp. 397-415, 1996.

[29] H. Choset and J. W. Burdick, "Sensor based planning: The hierarchical generalized Voronoi graph," in Proc. Workshop on Algorithmic Foundations of Robotics, Toulouse, France, 1996.

[30] I. J. Cox, "Blanche - an experiment in guidance and navigation of an autonomous robot vehicle," IEEE Trans. on Robotics and Automation, vol. 7, no. $2,1991$.

[31] I. J. Cox and J. J. Leonard, "Probabilistic data association for dynamic world modeling: A multiple hypothesis approach," in Int. Conf. on Advanced Robotics, Pisa, Italy, 1991, pp. 1287-1294.

[32] G. D'Agostini, "Overcoming priors anxiety," in Bayesian Methods in the Sciences, J. M. Bernardo, Ed. Madrid, Spain: Real Academia de Ciencias, 1999, 
vol. 93, no. 3, special Issue of Rev. Acad. Cien. Madrid.

[33] J. F. G. de Freitas, M. Noranjan, A. H. Gee, and A. Doucet, "Sequential Monte Carlo methods to train neural network models," Neural Computation, vol. 12, pp. $955-993,2000$.

[34] F. Dellaert, D. Fox, W. Burgard, and S. Thrun, "Monte Carlo localization for mobile robots," in Proc. of IEEE Int. Conf. on Robotics and Automation, vol. 2, 1999, pp. 1322-1328.

[35] A. P. Dempster, A. N. Laird, and D. B. Rubin, "Maximum likelihood from incomplete data via the EM algorithm," Journal of the Royal Statistical Society, vol. 39 , no. 1 , pp. 1-38, 1997.

[36] G. Dissanayake, P. Newman, S. Clark, H. F. Durrant-Whyte, and M. Csorba, "A solution to simultaneous localization and map building (SLAM) problem," IEEE Journal of Robotics and Automation, vol. 17, no. 3, 2001.

[37] A. Doucet, N. de Freitas, and N. Gordon, Eds., Sequential Monte Carlo Methods in Practice. New York, NY: Springer-Verlag, 2001.

[38] A. Doucet, S. Godsill, and C. Andrieu, "On sequential Monte Carlo sampling methods for Bayesian filtering," Statistics and Computing, vol. 10, pp. 197-208, 2000.

[39] D. Dubois and H. Prade, "A set-theoretic view on belief functions: Logical operations and approximations by fuzzy sets," International Journal of General Systems, vol. 12, pp. 193-226, 1986.

[40] H. Durrant-Whyte, S. Majumder, S. Thrun, M. de Battista, and S. Scheding, "A Bayesian algorithm for simultaneous localization and map building," in Proc. of the 10th Int. Symposium of Robotics Research (ISRR'01), 2001.

[41] A. Elfes, "Sonar-based real-world mapping and navigation," IEEE Journal of 
Robotics and Automation, pp. 249-265, 1987.

[42] S. Engelson and D. McDermott, "Error correction in mobile robot map learning," in Proc. of the 1992 IEEE Int. Conf. on Robotics and Automation, 1992, pp. 2555-2560.

[43] D. Fox, "Markov localization: A probabilistic framework for mobile robot localization and navigation," Ph.D. dissertation, Institute of Computer Science III, University of Bohn, Germany, 1998.

[44] _ , "Adapting the sample size in particle filters through kld-sampling," in Proc. of Neural Information Processing Systems (NIPS'01), 2001.

[45] F. Gambino and G. Oriolo, "A comparison of three uncertainty calculus techniques for ultrasonic map building," in Proc. SPIE Int. Symp. Aerospace/Defense Sensing and Control, 1996, pp. 249-260.

[46] R. Gartshore, A. Aguado, and C. Galambos, "Incremental map building using an occupancy grid for an autonomous monocular robot," in Seventh Int. Conf. on Control, Automation, Robotics And Vision (ICARCV02), vol. 2, 2002, pp. 613-618.

[47] J. Geweke, "Bayesian inference in econometric models using Monte Carlo integration," Econometrica, vol. 57, no. 6, pp. 1317-1339, 1989.

[48] W. R. Gilks and C. Berzuini, "Following a moving target - Monte Carlo inference for dynamic Bayesian models," Journal of Royal Statistical Society, vol. 63, pp. 127-146, 2001.

[49] S. Godsill, A. Doucet, and M. West, "Methodology for Monte Carlo smoothing with application to time-varying autoregresssions," in Proc. Int. Symp. Frontiers Time Series Modeling, 2000.

[50] N. J. Gordon, D. J. Salmond, and A. F. M. Smith, "Novel approach to 
nonlinear/non-Gaussian Bayesian state estimation," in IEE Proc. $F$, vol. 140, 1993, pp. 107-113.

[51] G. Grisetti, L. Iocchi, and D. Nardi, "Global Hough localization for mobile robots in polygonal environments," in Proc. of International Conference on Robotics and Automation (ICRA02), vol. 1, Washington DC, USA, 2002, pp. 353-358.

[52] H. M. Gross, A. Koenig, H. Boehme, and C. Schroeter, "Vision-based Monte Carlo self-localization for a mobile service robot acting as shopping assistant in a home store," in Proc. of the IEEE Int. Conf. on Intelligent Robots and System, vol. 1, 2002, pp. 256-262.

[53] J. E. Guivant and E. M. Nebot, "Optimization of the simultaneous localization and map-building algorithm for real-time implementation," IEEE Trans. on Robotics and Automation, vol. 17, no. 3, pp. 242-257, 2001.

[54] J. Gutmann and D. Fox, "An experimental comparison of localization methods continued," in Proc. of the IEEE Int. Conf. on Intelligent Robots and Systems, vol. 1, 2002, pp. 454-459.

[55] J. S. Gutmann and K. Konolige, "Incremental mapping of large cyclic environments," in Proc. of the IEEE Int. Symposium on Computational Intelligence in Robotics and Automation (CIRA), 2000.

[56] D. Guzzoni, A. Cheyer, L. Julia, and K. Konolige, "Many robots make short work," AI Magazine, vol. 18, no. 1, pp. 55-64, 1997.

[57] J. Hertzberg and F. Kirchner, "Landmark-based autonomous navigation in sewerage pipes," in Proc. of the First Euromicro Workshop on Advanced Mobile Robots (EUROBOT '96). IEEE Computer Society Press, 1996. [Online]. Available: citeseer.ist.psu.edu/166075.html 
[58] L. Iocchi, D. Mastrantuono, and D. Nardi, "A probabilistic approach to Hough localization," in Int. Conf. on Robotics and Automation (ICRA), Seoul, Korea, 2001.

[59] L. Iocchi and D. Nardi, "Hough transform based localization for mobile robots," in Advances in Intelligent Systems and Computer Science, N. Mastorakis, Ed. World Scientific Engineering Society, 1999.

[60] M. Isard and A. Blake, "CONDENSATION - conditional density propagation for visual tracking," Int. Journal of Computer Vision, vol. 29, no. 1, pp. 5-28, 1998.

[61] A. K. Jain, M. N. Murty, and P. J. Flynn, "Data clustering: A review," $A C M$ Computing Surveys, vol. 31, no. 3, pp. 264-323, Sept. 1999.

[62] P. Jensfelt and S. Kristensen, "Active global localization for a mobile robot using multiple hypothesis tracking," IEEE Trans. on Robotics and Automation, vol. 17 , no. 5, pp. 748-760, Oct. 2001.

[63] K. Kanazawa, D. Koller, and S. Russell, "Stochastic simulation algorithms for dynamic probabilistic networks," in Proceedings of the 11th Annual Conference on Uncertainty in AI (UAI), Montreal, Canada, Aug. 1995, pp. 346-351.

[64] J. E. Kerrich, An Experimental Introduction to the Theory of Probability. Copenhagen, Denmark: J. Jorgensen, 1946.

[65] G. Kitagawa, "Monte Carlo filter and smoother for non-Gaussian non-linear state space models," Journal of Computational and Graphical Statistics, vol. 5, no. 1, pp. 1-25, 1996.

[66] A. Kong, J. S. Liu, and W. H. Wong, "Sequential imputations and Bayesian missing data problems," Journal of the American Statistical Association, vol. 89, pp. 278-288, 1994. 
[67] D. Kortenkamp and T. Weymouth, "Topological mapping for mobile robots using a combination of sonar and vision sensing," in Proc. of the Twelfth National Conf. on Artificial Intelligence, 1994, pp. 979-984.

[68] B. Kuipers and Y. T. Byun, "A robot exploration and mapping strategy based on a semantic hierarchy of spatial representations," Journal of Robotics and Autonomous Systems, vol. 8, pp. 47-63, 1991.

[69] A. M. Ladd, K. E. Bekris, G. Marceau, A. Rudys, D. S. Wallach, and L. E. Kavraki, "Using wireless ethernet for localization," in Proc. of the IEEE Int. Conf. on Intelligent Robots and Systems, 2002.

[70] S. Lenser and M. Veloso, "Sensor resetting localization for poorly modelled mobile robots," in Proc. of the IEEE Int. Conf. on Robotics and Automation, vol. 2, 2000, pp. 1225-1232.

[71] J. Leonard and H. Feder, "Decoupled stochastic mapping [for mobile robot \& AUV navigation]," IEEE Journal of Oceanic Engineering, vol. 66, no. 4, pp. $561-571,2001$.

[72] J. J. Leonard and H. F. Durrant-Whyte, "Mobile robot localization by tracking geometric beacons," IEEE Trans. on Robotics and Automation, vol. 7, no. 3, pp. 376-382, 1991.

[73] — - "Simultaneous map building and localization for an autonomous mobile robot," in Proc. of IEEE/RSJ Int. Workshop on Intelligent Robots and Systems '91, vol. 3, 1991, pp. 1442-1447.

[74] J. J. Leonard and H. J. S. Feder, "A computationally efficient method for largescale concurrent mapping and localization," in Proc. of the Ninth Int. Symposium on Robotics Research, 1999.

[75] J. S. Liu and R. Chen, "Blind deconvolution via sequential imputation," Journal 
of the American Statistical Association, vol. 90, pp. 567-576, 1995.

[76] — - "Sequential Monte Carlo methods for dynamical systems," Journal of American Statistical Association, vol. 93, pp. 1032-1044, 1998.

[77] T. Logsdon, Understanding the Navstar: GPS, GIS and IVHS, 2nd ed. New York, NY: Van Nostrand Reinhold, 1995.

[78] F. Lu and E. Milios, "Globally consistent range scan alignment for environment mapping," Autonomous Robots, vol. 4, 1997.

[79] V. J. Lumelsky, "Guest editorial," IEEE Journal of Robotics and Automation, vol. RA-3, no. 3, 1987.

[80] K. Lynch, The Image of the City. MIT Press, 1960.

[81] M. J. Matarić, "Environment learning using a distributed representation," in Proc. of IEEE Int. Conf. on Robotics and Automation, vol. 1, 1990, pp. 402-406.

[82] L. H. Matthies and A. Elfes, "Sensor integration for robot navigation: Combining sonar and stereo range data in a grid-based representation," in Proc. of 26th IEEE Decision and Control Conference, 1987.

[83] P. S. Maybeck, Stochastic models, estimation, and control. New York, NY: Academic Press, 1979, vol. 1.

[84] J. D. R. Millan and A. Arleo, "Neural network learning of variable grid-based maps for the autonomous navigation of robots," in Proc. of Computational Intelligence in Robotics and Automation, 1997, pp. 40-45.

[85] A. Milstein, 2004, private communication.

[86] A. Milstein, J. N. Sánchez, and E. T. Williamson, "Robust global localization using clustered particle filtering," in Proceedings of the Eighteenth National Conference on Artificial Intelligence, Edmonton, Alberta, Canada, July 2002.

[87] M. Montemerlo, S. Thrun, D. Koller, and B. Wegbreit, "FastSLAM: A factored 
solution to the simultaneous localization and mapping problem," in Proc. of the 18th AAAI National Conf. on Artificial Intelligence, 2002, pp. 593-598.

[88] — _ "FastSLAM 2.0: An improved particle filtering algorithm for simultaneous localization and mapping that provably converges," in Proc. of the 18th Joint Conf. on Artificial Intelligence, 2003, pp. 907-912.

[89] H. P. Moravec, "Sensor fusion in certainty grids for mobile robots," AI magazine, vol. 9, no. 2, pp. 61-74, 1988.

[90] H. Moravec and A. Elfes, "High resolution maps from wide angle sonar," in Proc. IEEE Int. Conf. Robotics and Automation, 1985.

[91] K. Murphy and S. Russell, "Rao-blackwellized particle filtering for dynamic Bayesian networks," in Sequential Monte Carlo Methods in Practice, A. Doucet, N. de Freitas, and N. Gordon, Eds. Springer Verlag, 2001, pp. 499-516.

[92] S. K. Nayar, H. Murase, and S. A. Nene, "Learning, positioning, and tracking visual appearance," in Proc. of the IEEE International Conf. on Robotics and Automation, San Diego, CA, USA, May 1994, pp. 3237-3246.

[93] J. Neira and J. D. Tardos, "Data association in stochastic mapping using the joint compatibility test," IEEE Trans. of Robotics and Automation, pp. 890$897,2001$.

[94] N.J.Nilsson, "Shakey the robot," SRI, Menlo Park, CA, SRI Technical Note $323,1984$.

[95] I. Nourbakhsh, R. Power, and S. Birchfield, "DERVISH an office-navigating robot," AI Magazine, vol. 16, no. 2, pp. 53-60, 1995.

[96] C. F. Olson, "Subpixel localization and uncertainty estimation using occupancy grids," in Proc. of the 1999 IEEE Int. Conf. on Robotics 6 Automation, vol. 3, 1999, pp. 1987-1992. 
[97] D. Pagac, E. M. Nebot, and H. Durrant-Whyte, "An evidential approach to map-building for autonomous vehicles," IEEE Trans. on Robotics and Automation, vol. 14, no. 4, pp. 623-629, 1998.

[98] A. Papoulis and S. U. Pillai, Probability, Random Variables and Stochastic Processes, 4th ed. New York, NY, USA: McGraw-Hill, 2002.

[99] A. Pentland, B. Moghaddam, and T. Starner, "View-based and modular eigenspaces for face recognition," in Proc. of the IEEE Conf. on Computer Vision and Pattern Recognition. Seattle, WA, USA: IEEE Press, June 1994, pp. 84-90.

[100] J. Piaget and B. Inhelder, The Child's Conception of Space. London: Routledge \& Kegan Paul, 1971.

[101] D. Pierce and B. Kuipers, "Learning to explore and build maps," in Proc. of the Twelfth National Conf. on Artificial Intelligence, 1994, pp. 1264-1271.

[102] E. A. Puente, L. Moreno, M. A. Salichs, and D. Gachet, "Analysis of data fusion methods in certainty grids application to collision danger monitoring," in Proc. of IEEE Int. Conf. on Industrial Electronics, Controls \& Instrumentation, vol. 2, 1991, pp. 1133-1137.

[103] L. Rabiner and B. Juang, "An introduction to hidden Markov models," IEEE ASSP Magazine, vol. 3, no. 1, 1986.

[104] B. Ripley, Stochastic Simulation. New York, NY: Wiley, 1987.

[105] Official robocup website. The Robocup Federation. [Online]. Available: http://www.robocup.org

[106] T. Röfer and M. Jüngel, "Vision-based fast and reactive Monte-Carlo localization," in Proc. of the IEEE Int. Conf. on Robotics and Automation, vol. 1, 2003, pp. 856-861. 
[107] D. B. Rubin, "Using the SIR algorithm to simulate posterior distributions," in Bayesian Statistics, 3rd ed., J. M. Bernardo, M. H. DeGroot, D. V. Lindley, and A. F. M. Smith, Eds. Oxford University Press, 1988, pp. 395-402.

[108] S. J. Russell and P. Norvig, Artificial Intelligence: A Modern Approach. Upper Saddle River, NJ: Prentice Hall, 1995.

[109] B. Schiele and J. Crowley, "A comparison of position estimation techniques using occupancy grids," in Proc. of the 1994 IEEE Int. Conf. on Robotics and Automation, San Diego, CA, May 1994, pp. 1628-1634.

[110] A. C. Schultz and W. Adams, "Continuous localization using evidence grids," Naval Research Laboratory, Washington DC, NCARAI Report AIC-96-007, 1996.

[111] Toss Out the Toss-Up: Bias in heads-or-tails, vol. 165, no. 9, Science News, Feb. 2004. [Online]. Available: http://www.sciencenews.org/articles/20040228/fob2.asp

[112] G. Shafer, A Mathematical Theory of Evidence. Princeton, NJ: Princeton University Press, 1976.

[113] — , "The unity and diversity of probability," Statistical Science, vol. 5, pp. 435-462, 1990.

[114] _ - "Can the various meanings of probability be reconciled?" in A Handbook for Data Analysis in the Behavioral Sciences: Methodological Issues, G. Keren and C. Lewis, Eds. Hillsdale, New Jersey: Lawrence Erlbaum Inc., 1992, pp. 165-196.

[115] — , "What is probability?" in Perspectives on Contemporary Statistics, ser. MAA Notes, D. C. Hoaglin and D. S. Moore, Eds., 1992, no. 21, pp. 93-105.

[116] H. Shatkay and L. Kaelbling, "Learning topological maps with weak local odo- 
metric information," in Proc. of IJCAI-97, 1997.

[117] A. W. Siegel and S. H. White, "The development of spatial representations of large-scale environments," in Advances in Child Development and Behavior, H. Reese, Ed. New York, NY: Academic Press, 1975, vol. 10.

[118] R. Sim, "Mobile robot localisation using learned landmarks," Master's thesis, Department of Computer Science, McGill University, Montréal, Canada, July 1998.

[119] R. Simmons and S. Koenig, "Probabilistic robot navigation in partially observable environments," in Proc. of the Int. Joint Conf. on Artificial Intelligence, 1995.

[120] R. Smith, M. Self, and P. Cheeseman, "Estimating uncertain spatial relationships in robotics," in Autonomous Robot Vehicles, I. J. Cox and G. T. Wilfong, Eds. Springer-Verlag, 1990, pp. 167-193.

[121] R. C. Smith and P. Cheeseman, "On the representation and estimation of spatial uncertainty," SRI, Tech. Rep. TR 4760 \& 7239, 1985.

[122] S. Thrun, "Learning maps for indoor mobile robot navigation," Artificial Intelligence, vol. 99, no. 1, pp. 21-71, 1998.

[123] _ "A probabilistic online mapping algorithm for teams of mobile robots," Int. Journal of Robotics Research, vol. 20, no. 5, pp. 335-363, 2001.

[124] S. Thrun, M. Beetz, M. Bennewitz, W. Burgard, A. B. Cremers, F. Dellaert, D. Fox, D. Hähnel, C. Rosenberg, N. Roy, J. Schulte, and D. Schulz, "Probabilistic algorithms and the interactive museum tour-guide robot minerva," in Int. Journal of Robotics Research, vol. 19, 2000, pp. 972-999.

[125] S. Thrun, W. Burgard, and D. Fox, "A real-time algorithm for mobile robot mapping with applications to multi-robot and 3D mapping," in Proc. of the 
IEEE Int. Conf. on Robotics and Automation (ICRA), 2000.

[126] S. Thrun, D. Fox, and W. Burgard, "A probabilistic approach to concurrent mapping and localization for mobile robots," Machine Learning, vol. 31, pp. 29-53, 1998.

[127] S. Thrun, D. Fox, W. Burgard, and F. Dellaert, "Robust Monte Carlo localization for mobile robots," in Artificial Intelligence Journal, 2001.

[128] T. Tsumura, "Survey of automated guided vehicle in japanese factory," in Proceedings of the 1986 IEEE Int. Conf. on Robotics and Automation, vol. 3, Apr. 1986, pp. 1329-1334.

[129] M. Turk and A. Pentland, "Face processing: Models for recognition," in Intelligent Robots and Computer Vision VIII: Algorithms and Techniques, Philadelphia, PA, USA, 1989, pp. 22-32.

[130] R. Ueda, T. Fukase, Y. Kobayashi, T. Arai, H. Yuasa, and J. Ota, "Uniform Monte Carlo localization - fast and robust self-localization method for mobile robots," in Proc. of the IEEE Int. Conf. on Robotics and Automation, vol. 2, 2002, pp. 1353-1358.

[131] N. Vandapel and R. Chatila, "Affine trackability for landmark selection in natural environment," in Proc. of the 2002 IEEE/RSJ Int. Conf. on Intelligent Robots and Systems, Lausanne, Switzerland, Oct 2002.

[132] J. Vermaak, A. Doucet, and P. Pérez, "Maintaining multi-modality through mixture tracking," in Int. Conf. on Computer Vision, ICCV'03, Nice, France, June 2003.

[133] B. Yamauchi and R. Beer, "Spatial learning for navigation in dynamic environments," IEEE Trans. on Systems, Man and Cybernetics - Part B: Cybernetics, Special Issue on Learning Autonomous Robots, 1996. 
[134] B. Yamauchi and P. Langley, "Place recognition in dynamic environments," Journal of Robotic Systems, vol. 14, pp. 107-120, 1997.

[135] B. Yamauchi, P. Langley, A. C. Schultz, J. Grefenstette, and W. Adams, "Magellan: An integrated adaptive architecture for mobile robots," Institute for the Study of Learning and Expertise (ISLE), Palo Alto, CA, Tech. Rep. 98-2, 1998.

[136] B. Yamauchi, A. Schultz, and W. Adams, "Mobile robot exploration and mapbuilding with continuous localization," in Proc. of the IEEE Int. Conf. on Robotics \& Automation, 1998.

[137] T. Yang, "Software design and strategy for an autonomous mobile robot," B. Eng thesis, Department of Electronics, Carleton University, Ottawa, Canada, 2002, for a robot in the Roboflag competition.

[138] L. A. Zadeh, "Review of shafer's a mathematical theory of evidence," AI Magazine, vol. 5, pp. 81-83, 1984.

[139] V. S. Zaritskii, V. B. Svetnik, and L. I. Shimelevich, "Monte Carlo technique in problems of optimal data processing," in Automation and Remote Control, vol. 12, 1975, pp. 95-103.

[140] U. R. Zimmer, "Robust world-modeling and navigation in a real world," Neurocomputing, vol. 13, no. 2-4, 1996. 


\section{Appendix A: Simulator Software}

The simulator created to simulate the results in this thesis is written in Java and $\mathrm{C} / \mathrm{C}++$. This simulator is briefly described in Section 4 , and is discussed in more detail here.

The simulator uses many software technologies as listed below:

- Java3D

- Java Foundation Classes/Swing (JFC/Swing)

- Java Native Interface (JNI)

- $\mathrm{C} / \mathrm{C}++$

- GNU Scientific Library (GSL)

- Java Compiler Compiler (JavaCC)

In designing the simulator, an early decision was made to include a rendering component so that the actual environment simulated can be conveyed to the user in an intuitive manner. Different rendering technologies such as OpenGL, DirectX, and Java3D were evaluated, and Java3D was selected for its powerful 3D capabilities as well as its integration with the Java programming language. JFC/Swing was used to 
create the graphical user interface (GUI) so that the user can control the simulation in a non-archaic manner. Having the whole project as a Java program simplified implementation. Although every effort was made to keep the implementation simple, it was soon evident that Java did not provide all the functionality required for the simulator, most notably, the scientific math computation capabilities. If the project was to remain as a pure Java program, capabilities such as solving for eigenvalues or other matrix operations needed to be implemented from scratch. The simulator was subsequently made into a cross-language program using the JNI technology. In doing so, the much touted cross platform compatibility feature of Java programs is lost. Using JNI allows Java to interface with compiled code native to a specific platform, Microsoft Windows in this case. In this case, the simulator is able to access existing $\mathrm{C}$ libraries that are freely available. The $\mathrm{C}$ library of interest is the GSL. The GSL contains a multitude of functionality useful for scientific programs. The features of interest in our application are matrix manipulation, linear algebra, and random number generation using popular distributions. As a result, much of the code that required the use of these capabilities are written in $\mathrm{C} / \mathrm{C}++$. As the simulator developed, a substantial number of capabilities were added to the simulator, each with their own parameter settings. Many of these settings were specified with a configuration text file, and still are. However, the code that read the configuration file has been upgraded to use JavaCC. JavaCC facilitates the reading of a well defined configuration file, and is easily extendable by modifying the grammar of the configuration file.

With the brief implementation history of the simulator, the general software architecture of the simulator is as shown in Figure A-1. The Java portion is primarily responsible for the rendering, the GUI, and the high level coordination of the program. The native portion is responsible for low level subsystems of the simulator, 
the mapping and localisation components in particular. Much of the data related to mapping and localisation is in the native portion of the program. The data include the grid map created, and the samples of the particle filters. As such, there is an inefficient duplication process when the data is needed in the Java portion of the program, especially for display purposes. Also, the architecture of the Java portion is mirrored in the native portion. There are many stubs created in both portions of the code. The simulator would be more efficient written fully in $\mathrm{C} / \mathrm{C}++$.

\section{A.1 Graphical Interface}

The GUI interacts with the user by giving many options which are available as part of the menu system, and the user can choose to open option panels and place it in the program's drawing area. Figure A-2 is a screenshot of the simulator simulating a test case for the UCPF-MCL method with a few open panels. The larger panel on the left shows the overhead 2-D view of the environment, with the estimated robot locations drawn on it. The larger panel on the right shows the virtual 3-D environment that simulates the real environment. The first panel below the larger panel on the right is the robot properties panel, and the second panel is the path planner panel. The different menu items and the different panels of the software are described below:

File $\rightarrow$ Exit: This menu option quits the simulator.

Simulation $\rightarrow$ Start: This menu option starts the simulation as defined.

Simulation $\rightarrow$ Pause: This menu option pauses the simulation at the current time step. 


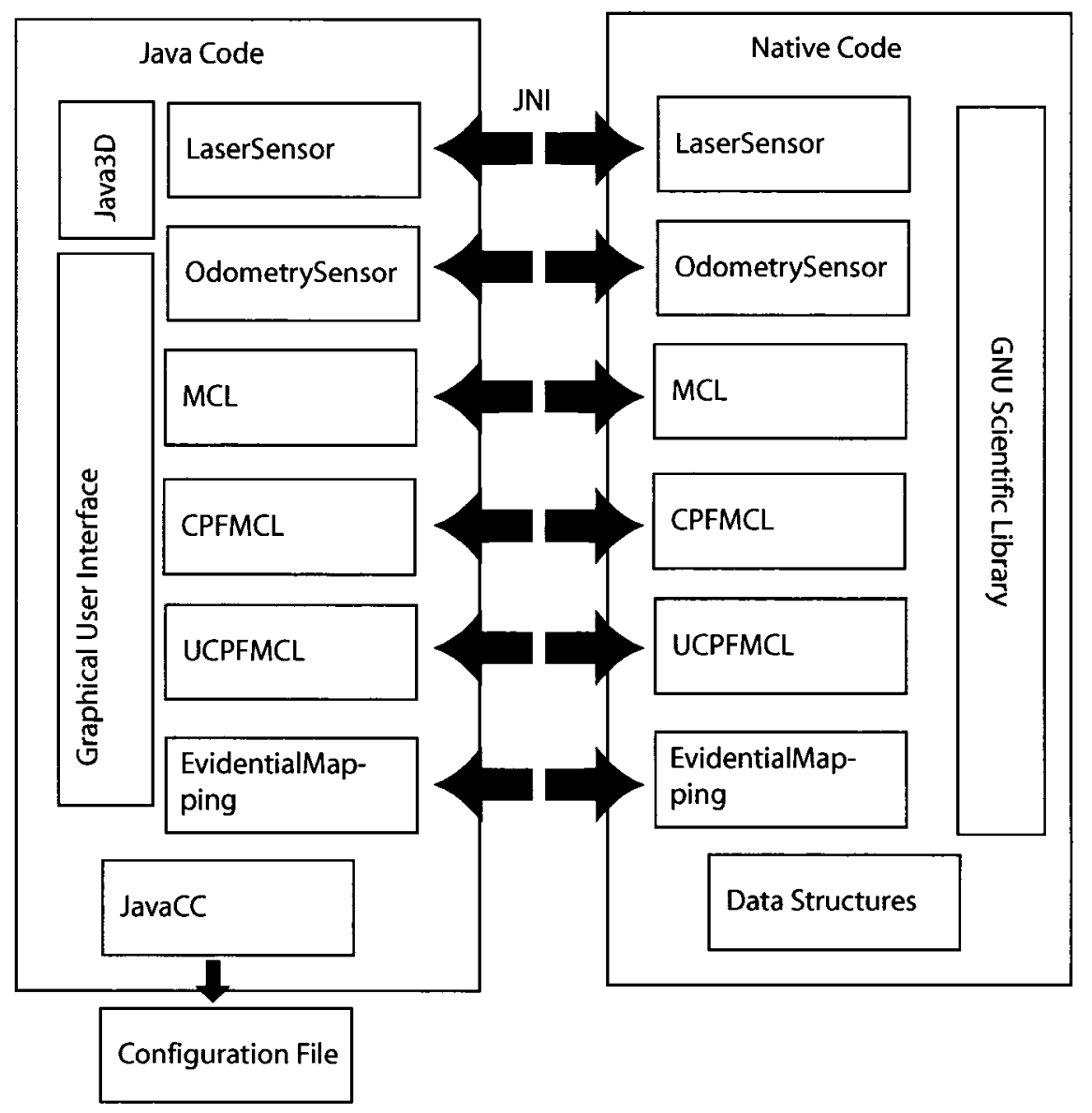

Figure A-1: Detailed Software Architecture

Simulation $\rightarrow$ Toggle Auto Stop: This menu option, when enabled, pauses the simulation after every localisation resampling process.

Simulation $\rightarrow$ Toggle Attended Stop: This menu option does not currently perform anything useful.

Simulation $\rightarrow$ Toggle Manual Render: This menu option causes the rendering for the 3-D environment to only be re-drawn when there is a change in the simulated 


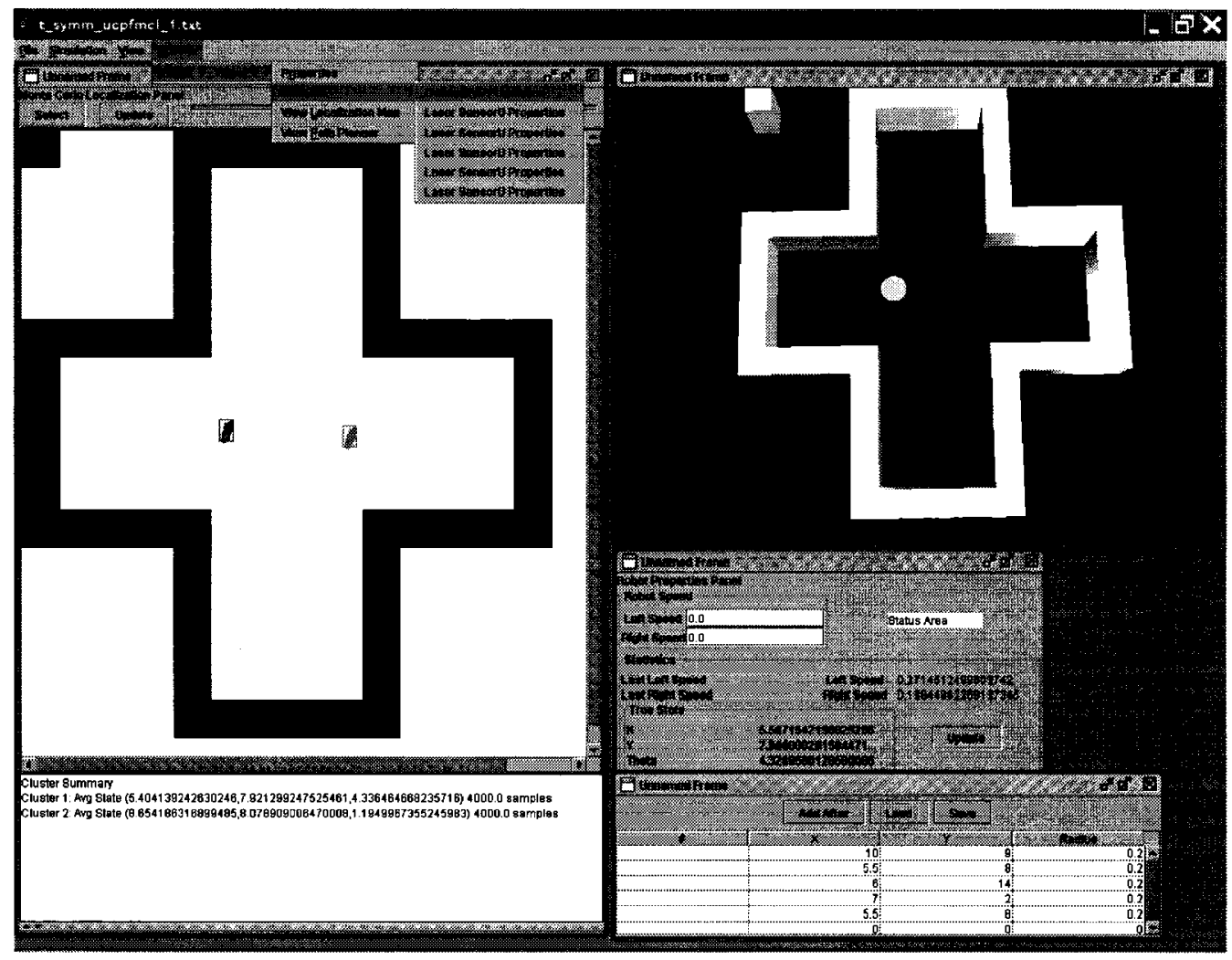

Figure A-2: Simulator Screenshot

world.

View $\rightarrow$ Adjustable View: This menu option opens up a 3-D rendered view of the simulated environment. This view may be adjusted using the page-up, pagedown, and the arrow keys to move around the environment.

View $\rightarrow$ Top View: This menu option opens up an overhead 3-D rendered view of the simulated environment. This view is not adjustable.

Actions $\rightarrow$ Robot: For each robot created in the environment, a menu option is created within this menu containing options for the robot. Figure A-2 shows some options the robot may have available for the user to choose. 
Properties: This option opens the robot properties panel where the user can specify the speed of the individual drive wheels of the robot, and see the instantaneous speed of each wheel. Also available is the true state of the robot in the environment.

Sensors: This menu contains a sub-menu option for each sensor, or type of sensor installed on the robot. In the simulator, it would be either the odometry or laser sensor. Selecting the sub-menu options brings up a panel that allows the user to see statistics, sensor measurements, or enable the debugging mode.

View Localization Map: This option is available when a localisation unit is installed on the robot, such as the UCPF-MCL unit. When activated, a panel is opened that draws a graphical view of the robot's estimated states.

View Mapping Progress: This option is available when a mapping unit is installed on the robot, the only one currently available being the EvidentialMapping unit. When activated, a panel is opened that draws a graphical view of the robot's current map of the world.

View Path Planner: This option opens a panel that allows the user to specify absolute movement paths.

\section{A.2 Configuration}

The simulator has many tunable options to specify a simulation. Although some options may be changed online through the GUI, other options are specified in a configuration file that is loaded at simulator initialisation. A sample annotated configuration file is given in the next section. 


\section{A.2.1 Configuration File}

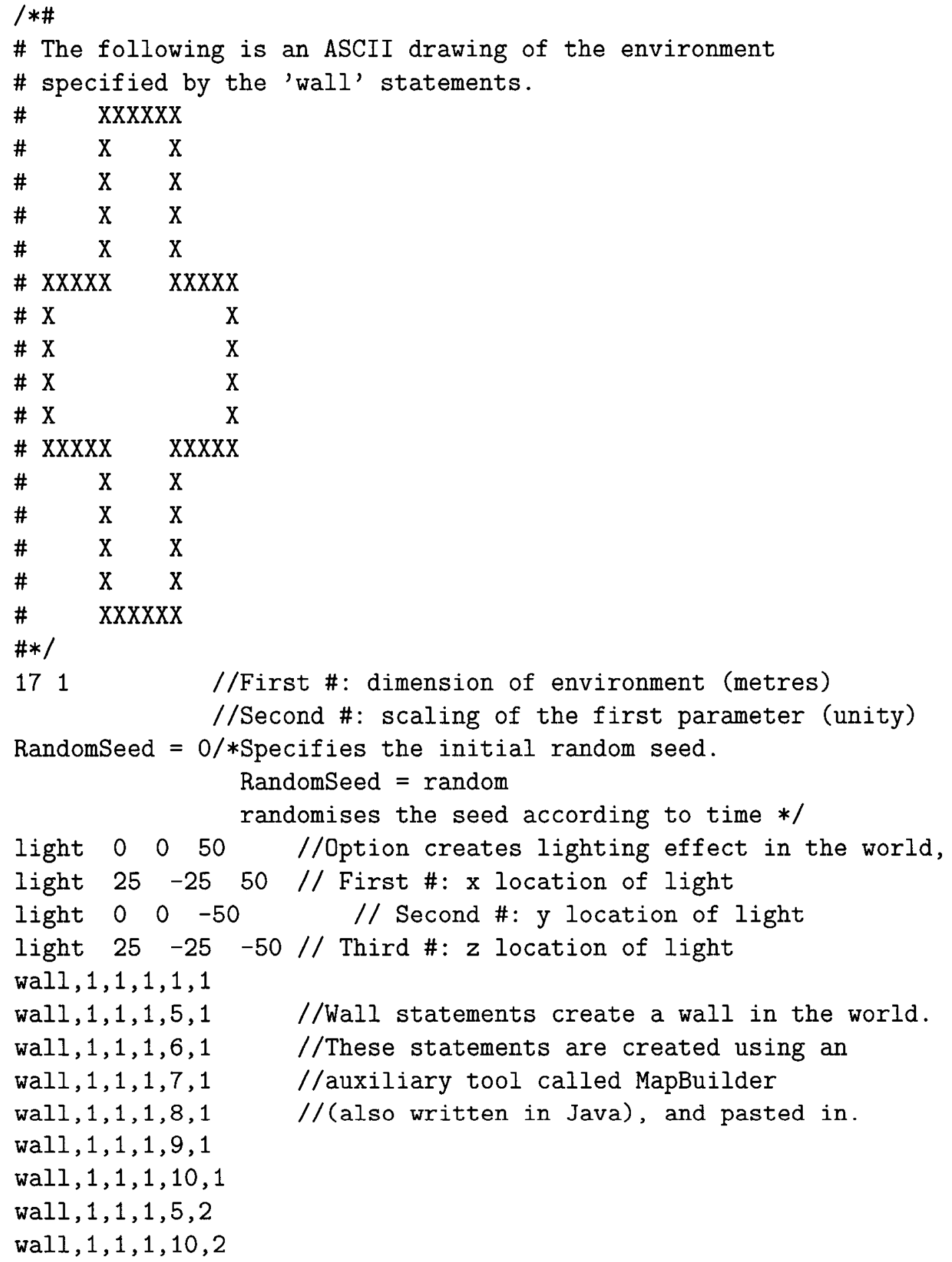




$$
\begin{aligned}
& \text { wall } 1,1,1,5,3 \\
& \text { wall , 1, 1, 1, 10, } 3 \\
& \text { wall , 1, 1, 1, 5, } 4 \\
& \text { wall }, 1,1,1,10,4 \\
& \text { wall } 1,1,1,5,5 \\
& \text { wall }, 1,1,1,10,5 \\
& \text { wall } 1,1,1,1,6 \\
& \text { wall, } 1,1,1,2,6 \\
& \text { wall , } 1,1,1,3,6 \\
& \text { wall, 1,1,1,4,6 } \\
& \text { wall } 1,1,1,5,6 \\
& \text { wall } 1,1,1,10,6 \\
& \text { wall } 1,1,1,11,6 \\
& \text { wall }, 1,1,1,12,6 \\
& \text { wall , } 1,1,1,13,6 \\
& \text { wall } 1,1,1,14,6 \\
& \text { wall , 1, 1, 1, 1,7 } \\
& \text { wall } 1,1,1,14,7 \\
& \text { wall } 1,1,1,1,8 \\
& \text { wall } 1,1,1,14,8 \\
& \text { wall } 1,1,1,1,9 \\
& \text { wall } 1,1,1,14,9 \\
& \text { wall , 1, 1, 1, 1, } 10 \\
& \text { wall } 1,1,1,14,10 \\
& \text { wall } 1,1,1,1,11 \\
& \text { wall , } 1,1,1,2,11 \\
& \text { wall , 1, 1, 1, 3, } 11 \\
& \text { wall } 1,1,1,4,11 \\
& \text { wall , } 1,1,1,5,11 \\
& \text { wall , } 1,1,1,10,11 \\
& \text { wall } 1,1,1,11,11 \\
& \text { wall } 1,1,1,12,11 \\
& \text { wall }, 1,1,1,13,11 \\
& \text { wall , 1, 1, 1, 14, } 11 \\
& \text { wall , 1, 1, 1, 5, } 12 \\
& \text { wall } 1,1,1,10,12 \\
& \text { wall , 1, 1, 1, 5, } 13 \\
& \text { wall , 1, 1, 1, 10,13 } \\
& \text { wall , 1, 1, 1, 5, } 14 \\
& \text { wall } 1,1,1,10,14
\end{aligned}
$$




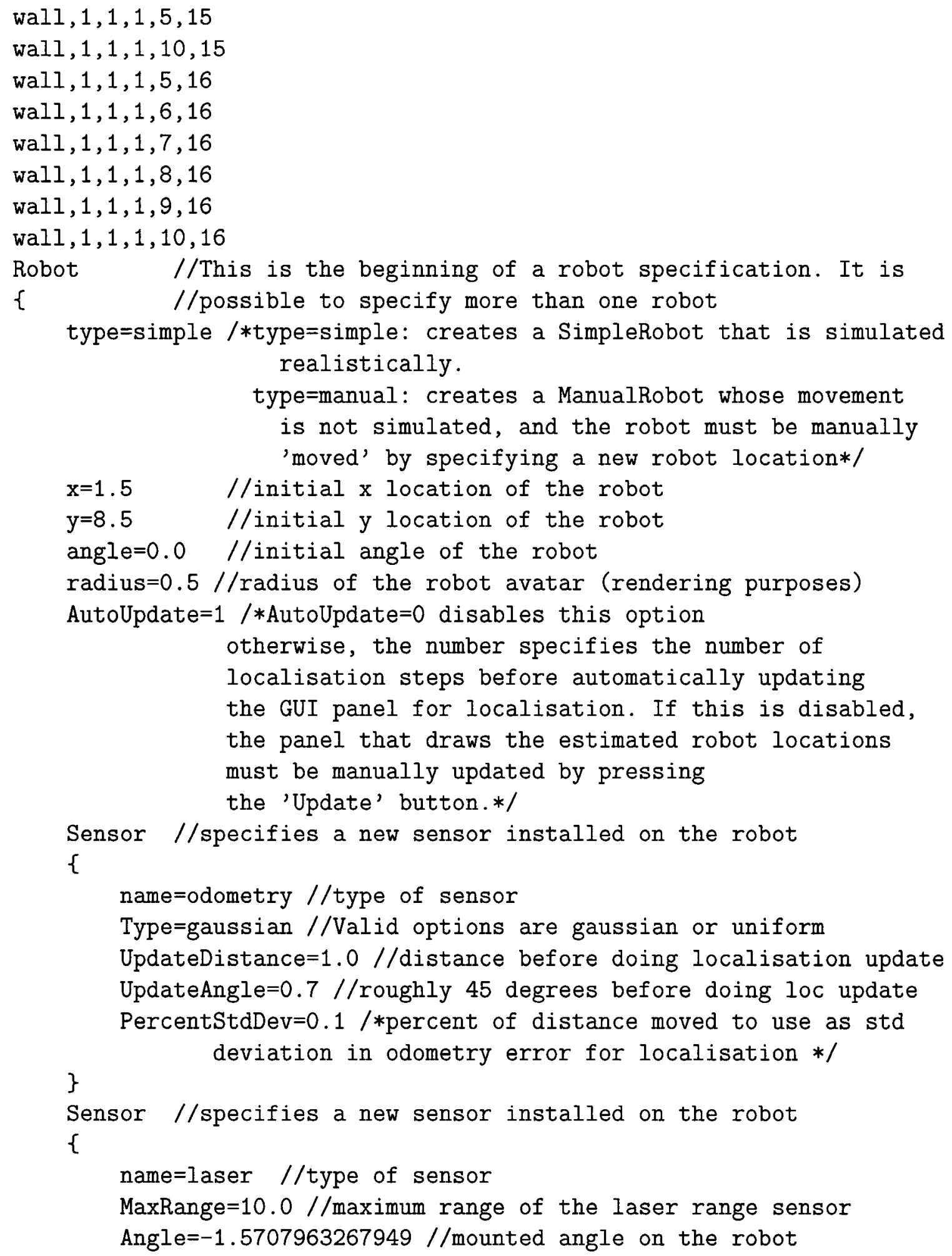




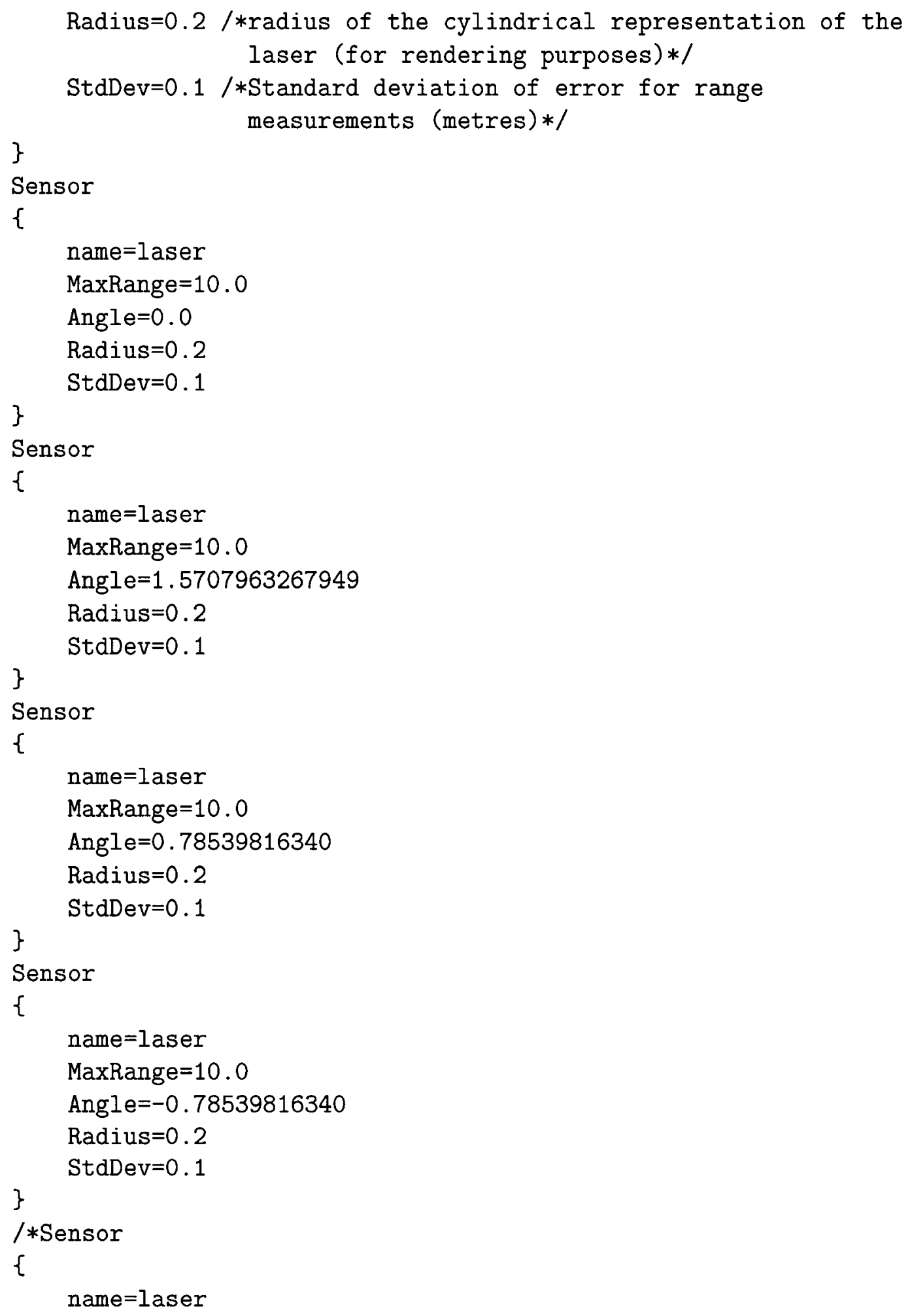




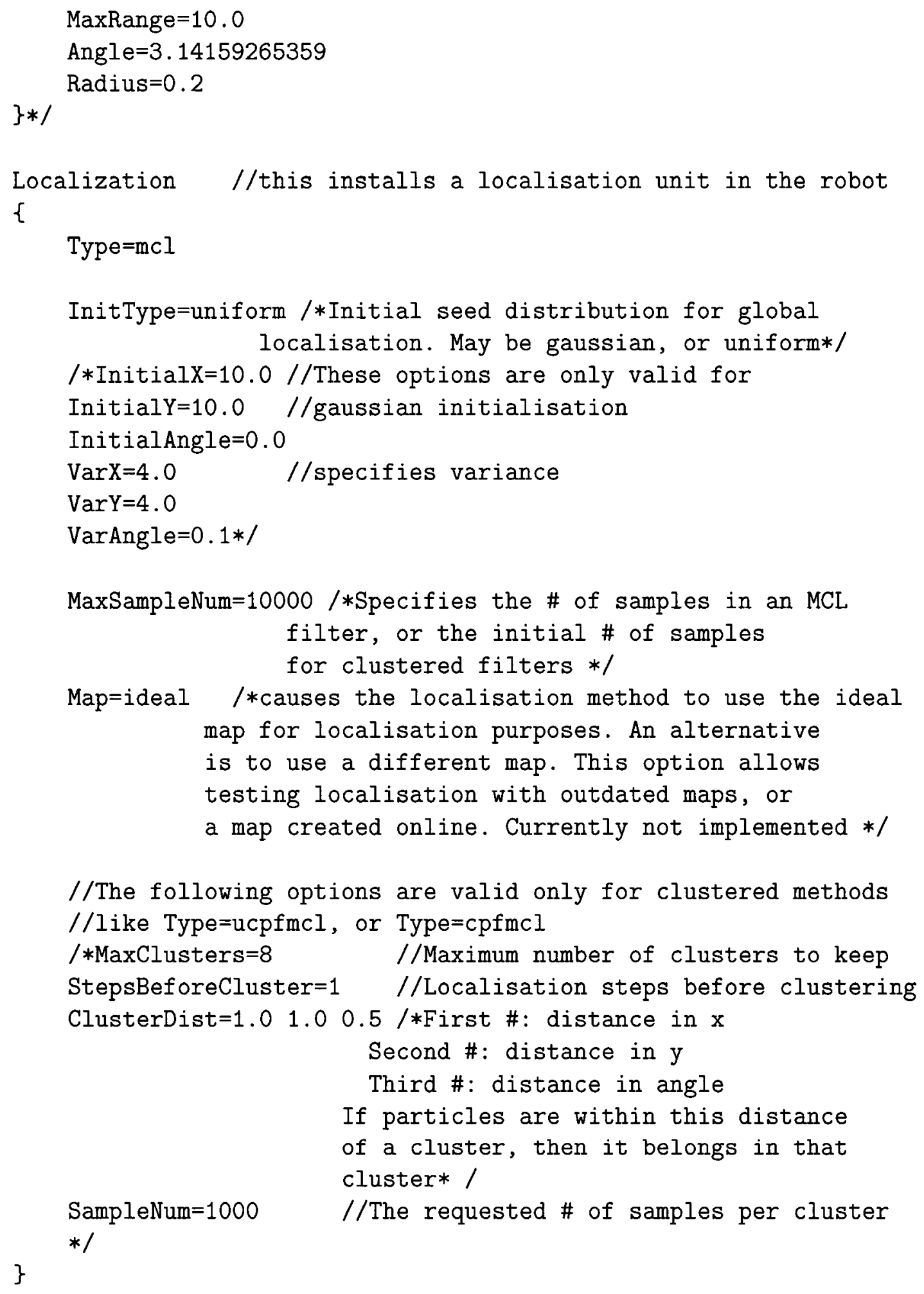


MotionModel //installs a motion model in the robot

\{

name=SimpleDrive //the only model currently available

Noise=DifferentialDrive $0.1 / /$ Specifies noise model to be based on a differential drive system. This is currently the only implemented noise model. The \# following specifies the percentage of speed to use as the std dev

\} of noisy motion $*$ /

PathPlanner //installs a path planning unit in the robot \{

type=SimplePathPlanner //the only available option MaxAngle $=5.0$

File="p_symm.txt" //specifies a file to load for robot's path AutoQuit /*auto quit the simulator after the path specified \} is run to completion */ 


\section{Appendix B: Simulation Results}

Tables of results for localisation are included in this appendix. Each table contains the calculated errors between different clusters of possible robot location and the true robot location for different localisation attempts for different test cases. In the tables, the column "\#" indicates the localisation attempt; the column "R" indicates the number of relocalisation attempts; the column "Most Likely Cluster" indicates the error between the most likely cluster according to its associated weights and the true location; the column "C" indicates the number of clusters kept by the filter; the column "Most Likely Sample" indicates the error between the most likely sample according to weights associated with samples of the filter and the true location; and the column "Closest Cluster" gives the error between the cluster that is closest to the true location, and the true location.

\section{B.1 Symmetric Map Results}

\section{B.1.1 MCL}


Table B-1: Error for Localisation Symmetric Map (MCL) Case 1.1

\begin{tabular}{|c|c|c|c|c|c|}
\hline \hline$\#$ & $\mathrm{R}$ & $\mathrm{C}$ & Most Likely Cluster & Most Likely Sample & Closest Cluster \\
\hline \hline 1 & 0 & 4 & $(4.1051,-5.7595,-1.5825)$ & $(4.0568,-5.7726,-1.5912)$ & $(-0.0772,0.0642,-1.6673)$ \\
\hline 2 & 0 & 3 & $(-0.0826,0.0639,-0.0201)$ & $(-0.1028,0.0634,-0.0201)$ & $(-0.0826,0.0639,-0.0201)$ \\
\hline 3 & 0 & 1 & $(0.0097,0.0838,-0.0191)$ & $(-0.0463,0.0826,-0.0191)$ & $(0.0097,0.0838,-0.0191)$ \\
\hline 4 & 0 & 1 & $(0.0478,0.1272,-0.0182)$ & $(0.0487,0.1296,-0.0178)$ & $(0.0478,0.1272,-0.0182)$ \\
\hline 5 & 0 & 1 & $(0.0564,0.1454,-0.018)$ & $(0.0335,0.1469,-0.0179)$ & $(0.0335,0.1469,-0.0179)$ \\
\hline 10 & 0 & 1 & $(-0.1891,0.4144,-0.0123)$ & $(-0.1304,0.4097,-0.0328)$ & $(-0.1304,0.4097,-0.0328)$ \\
\hline 15 & 0 & 1 & $(-0.1443,0.3284,-0.0386)$ & $(-0.1488,0.3299,-0.0371)$ & $(-0.1443,0.3284,-0.0386)$ \\
\hline 20 & 0 & 1 & $(0.0638,0.456,0.0398)$ & $(0.0619,0.4555,0.0387)$ & $(0.0619,0.4555,0.0387)$ \\
\hline 25 & 0 & 1 & $(0.1848,0.0366,0.0314)$ & $(0.1805,0.0231,0.0309)$ & $(0.1805,0.0231,0.0309)$ \\
\hline 30 & 0 & 1 & $(0.5203,-0.0931,0.2108)$ & $(0.558,-0.045,0.1897)$ & $(0.5203,-0.0931,0.2108)$ \\
\hline 35 & 0 & 1 & $(-0.1448,-0.0345,0.1424)$ & $(-0.1426,-0.0022,0.1424)$ & $(-0.1426,-0.0022,0.1424)$ \\
\hline 40 & 0 & 1 & $(-0.7806,0.2064,0.1427)$ & $(-0.7897,0.076,0.1427)$ & $(-0.7897,0.076,0.1427)$ \\
\hline 45 & 0 & 1 & $(-1.2542,0.136,0.1908)$ & $(-1.2557,0.1716,0.1893)$ & $(-1.2542,0.136,0.1908)$ \\
\hline 50 & 0 & 1 & $(-0.5288,1.2067,0.2463)$ & $(-0.5371,0.9604,0.2463)$ & $(-0.5371,0.9604,0.2463)$ \\
\hline 54 & 1 & 1 & $(2.8847,0.1259,-3.131)$ & $(2.8698,0.1644,-3.1313)$ & $(2.8698,0.1644,-3.1313)$ \\
\hline \hline
\end{tabular}

Table B-2: Error for Localisation Symmetric Map (MCL) Case 1.2

\begin{tabular}{|c|c|c|c|c|c|}
\hline \hline$\#$ & $\mathrm{R}$ & $\mathrm{C}$ & Most Likely Cluster & Most Likely Sample & Closest Cluster \\
\hline \hline 1 & 0 & 4 & $(4.0962,-5.7438,-1.5406)$ & $(4.9484,3.8404,-4.6886)$ & $(-0.0069,0.031,-2.901)$ \\
\hline 2 & 0 & 4 & $(3.2256,-4.5937,-1.6448)$ & $(3.2862,-4.493,-1.5741)$ & $(-0.1054,0.0208,-0.0329)$ \\
\hline 3 & 0 & 4 & $(0.0173,0.0835,-0.0193)$ & $(5.5881,-0.8844,-3.0926)$ & $(0.0173,0.0835,-0.0193)$ \\
\hline 4 & 0 & 2 & $(0.0434,0.1279,-0.0182)$ & $(0.0198,0.1234,-0.0182)$ & $(0.0198,0.1234,-0.0182)$ \\
\hline 5 & 0 & 1 & $(0.1079,0.151,-0.0181)$ & $(0.1261,0.158,-0.0183)$ & $(0.1079,0.151,-0.0181)$ \\
\hline 10 & 0 & 1 & $(-0.2165,0.4237,-0.0236)$ & $(-0.3276,0.3968,0.024)$ & $(-0.2165,0.4237,-0.0236)$ \\
\hline 15 & 0 & 1 & $(-0.2932,0.2649,-0.0649)$ & $(-0.213,0.3146,-0.0533)$ & $(-0.213,0.3146,-0.0533)$ \\
\hline 20 & 0 & 1 & $(0.0249,0.3874,0.0305)$ & $(0.0059,0.3479,0.0336)$ & $(0.0059,0.3479,0.0336)$ \\
\hline 25 & 0 & 1 & $(0.0879,0.1507,0.0142)$ & $(0.0508,0.1516,0.0069)$ & $(0.0508,0.1516,0.0069)$ \\
\hline 30 & 0 & 1 & $(0.4093,-0.0107,0.1301)$ & $(0.4547,0.1263,0.1559)$ & $(0.4093,-0.0107,0.1301)$ \\
\hline 35 & 0 & 1 & $(0.1394,-0.0343,0.0385)$ & $(0.166,0.0703,0.0196)$ & $(0.1394,-0.0343,0.0385)$ \\
\hline 40 & 0 & 1 & $(0.003,-0.2444,0.0341)$ & $(-0.0372,-0.1166,0.0453)$ & $(-0.0372,-0.1166,0.0453)$ \\
\hline 45 & 0 & 1 & $(-0.0907,-0.0921,0.0033)$ & $(-0.0628,-0.1336,-0.0433)$ & $(-0.0907,-0.0921,0.0033)$ \\
\hline 50 & 0 & 1 & $(-0.082,0.1735,0.0117)$ & $(-0.0895,0.2214,0.0132)$ & $(-0.082,0.1735,0.0117)$ \\
\hline 54 & 0 & 1 & $(-0.1297,0.0593,0.0073)$ & $(-0.17,0.0701,0.0086)$ & $(-0.1297,0.0593,0.0073)$ \\
\hline \hline
\end{tabular}


Table B-3: Error for Localisation Symmetric Map (MCL) Case 1.3

\begin{tabular}{|c|c|c|c|c|c|}
\hline \hline$\#$ & $\mathrm{R}$ & $\mathrm{C}$ & Most Likely Cluster & Most Likely Sample & Closest Cluster \\
\hline \hline 1 & 0 & 4 & $(4.0756,-5.7258,-1.4992)$ & $(3.1138,-6.2326,-1.0001)$ & $(0.0103,-0.0182,-3.1374)$ \\
\hline 2 & 0 & 4 & $(3.2426,-4.6257,-1.649)$ & $(3.2862,-4.4851,-1.5741)$ & $(-0.1019,-0.0936,-0.0586)$ \\
\hline 3 & 0 & 4 & $(0.0267,0.0486,-0.0309)$ & $(5.5881,-0.8844,-3.0926)$ & $(0.0267,0.0486,-0.0309)$ \\
\hline 4 & 0 & 1 & $(0.1815,0.0377,-0.159)$ & $(0.0659,0.1302,-0.0182)$ & $(0.0659,0.1302,-0.0182)$ \\
\hline 5 & 0 & 1 & $(0.1639,0.1013,-0.1066)$ & $(0.065,0.1484,-0.0185)$ & $(0.065,0.1484,-0.0185)$ \\
\hline 10 & 0 & 2 & $(-0.1213,0.3795,-0.0589)$ & $(-6.019,-2.3449,-3.2168)$ & $(-0.1213,0.3795,-0.0589)$ \\
\hline 15 & 0 & 1 & $(-2.0928,-1.1664,2.8651)$ & $(-2.0576,-1.3834,3.067)$ & $(-2.0928,-1.1664,2.8651)$ \\
\hline 20 & 0 & 1 & $(3.145,-0.1174,-3.1337)$ & $(3.1154,-0.0665,-3.1742)$ & $(3.145,-0.1174,-3.1337)$ \\
\hline 25 & 0 & 1 & $(2.4394,-9.464,-3.1319)$ & $(2.5273,-9.5158,-3.1565)$ & $(2.4394,-9.464,-3.1319)$ \\
\hline 30 & 0 & 1 & $(1.6083,-12.0427,3.1966)$ & $(1.7783,-11.9006,3.1994)$ & $(1.7783,-11.9006,3.1994)$ \\
\hline 35 & 0 & 1 & $(1.0674,-2.7887,3.1576)$ & $(1.171,-2.7468,3.1761)$ & $(1.0674,-2.7887,3.1576)$ \\
\hline 40 & 0 & 1 & $(0.5244,6.6293,3.1647)$ & $(0.4833,6.3907,3.1539)$ & $(0.4833,6.3907,3.1539)$ \\
\hline 45 & 0 & 1 & $(0.1479,12.3105,3.1371)$ & $(0.2118,12.5019,3.0956)$ & $(0.1479,12.3105,3.1371)$ \\
\hline 50 & 0 & 1 & $(1.3804,6.5649,-3.1339)$ & $(1.4277,6.4824,-3.1383)$ & $(1.4277,6.4824,-3.1383)$ \\
\hline 54 & 0 & 1 & $(2.9894,0.1849,-3.1416)$ & $(2.9642,0.1049,-3.1354)$ & $(2.9642,0.1049,-3.1354)$ \\
\hline \hline
\end{tabular}

Table B-4: Error for Localisation Symmetric Map (MCL) Case 1.4

\begin{tabular}{|c|c|c|c|c|c|}
\hline \hline$\#$ & $\mathrm{R}$ & $\mathrm{C}$ & Most Likely Cluster & Most Likely Sample & Closest Cluster \\
\hline \hline 1 & 0 & 4 & $(4.093,-5.7519,-1.5388)$ & $(4.9486,3.8391,-4.6859)$ & $(-0.0108,0.031,-2.8444)$ \\
\hline 2 & 0 & 4 & $(3.2278,-4.5884,-1.6402)$ & $(3.2897,-4.4967,-1.5675)$ & $(-0.0966,0.015,-0.0271)$ \\
\hline 3 & 0 & 4 & $(0.0198,0.0692,-0.0052)$ & $(5.5907,-0.8981,-3.0782)$ & $(0.0198,0.0692,-0.0052)$ \\
\hline 4 & 0 & 2 & $(0.0756,0.0716,0.0028)$ & $(0.0808,0.0717,0.0028)$ & $(0.0756,0.0716,0.0028)$ \\
\hline 5 & 0 & 1 & $(0.1018,0.1041,0.0026)$ & $(0.1443,0.1059,0.0026)$ & $(0.1018,0.1041,0.0026)$ \\
\hline 10 & 0 & 1 & $(-0.1854,0.3542,-0.0245)$ & $(-0.2623,0.3468,0.0205)$ & $(-0.1854,0.3542,-0.0245)$ \\
\hline 15 & 0 & 1 & $(-0.2658,0.1816,-0.0563)$ & $(-0.2521,0.1227,-0.0778)$ & $(-0.2521,0.1227,-0.0778)$ \\
\hline 20 & 0 & 1 & $(-0.0142,0.4064,0.0084)$ & $(-0.0217,0.3992,0.0022)$ & $(-0.0217,0.3992,0.0022)$ \\
\hline 25 & 0 & 1 & $(-0.0123,0.2519,0.0028)$ & $(0.0344,0.3173,0.0097)$ & $(-0.0123,0.2519,0.0028)$ \\
\hline 30 & 0 & 1 & $(0.29,0.0436,0.1212)$ & $(0.2905,-0.0207,0.0695)$ & $(0.2905,-0.0207,0.0695)$ \\
\hline 35 & 0 & 1 & $(0.0295,-0.0718,0.0338)$ & $(0.0691,-0.0836,0.0255)$ & $(0.0295,-0.0718,0.0338)$ \\
\hline 40 & 0 & 1 & $(-0.0247,-0.3018,0.0313)$ & $(0.0451,-0.3341,0.029)$ & $(-0.0247,-0.3018,0.0313)$ \\
\hline 45 & 0 & 1 & $(0.0993,-0.0266,-0.0032)$ & $(0.2747,-0.1502,-0.0762)$ & $(0.0993,-0.0266,-0.0032)$ \\
\hline 50 & 0 & 1 & $(-0.0379,0.1071,-0.0124)$ & $(-0.0584,0.0237,-0.0271)$ & $(-0.0584,0.0237,-0.0271)$ \\
\hline 54 & 0 & 1 & $(0.044,0.1537,-0.0099)$ & $(0.055,0.1234,-0.0211)$ & $(0.055,0.1234,-0.0211)$ \\
\hline \hline
\end{tabular}


Table B-5: Error for Localisation Symmetric Map (MCL) Case 1.5

\begin{tabular}{|c|c|c|c|c|c|}
\hline \hline$\#$ & $\mathrm{R}$ & $\mathrm{C}$ & Most Likely Cluster & Most Likely Sample & Closest Cluster \\
\hline \hline 1 & 0 & 4 & $(4.0712,-5.7302,-1.497)$ & $(3.1139,-6.2339,-0.9974)$ & $(0.0059,-0.0142,-3.1421)$ \\
\hline 2 & 0 & 4 & $(3.246,-4.6245,-1.6444)$ & $(3.2897,-4.4967,-1.5675)$ & $(-0.0975,-0.1007,-0.0534)$ \\
\hline 3 & 0 & 4 & $(0.0291,0.0234,-0.0215)$ & $(5.5907,-0.8981,-3.0782)$ & $(0.0291,0.0234,-0.0215)$ \\
\hline 4 & 0 & 1 & $(0.1535,0.0298,-0.0785)$ & $(0.0626,0.0713,0.0028)$ & $(0.0626,0.0713,0.0028)$ \\
\hline 5 & 0 & 1 & $(0.1434,0.102,-0.0044)$ & $(0.1228,0.1053,0.0025)$ & $(0.1228,0.1053,0.0025)$ \\
\hline 10 & 0 & 1 & $(-0.1912,0.3537,-0.0302)$ & $(-0.185,0.3844,-0.0644)$ & $(-0.1912,0.3537,-0.0302)$ \\
\hline 15 & 0 & 1 & $(-0.3051,0.1108,-0.0595)$ & $(-0.3083,0.0454,-0.0573)$ & $(-0.3083,0.0454,-0.0573)$ \\
\hline 20 & 0 & 1 & $(-0.0948,0.3358,0.0319)$ & $(-0.1097,0.3192,0.0634)$ & $(-0.1097,0.3192,0.0634)$ \\
\hline 25 & 0 & 1 & $(0.0158,0.4267,0.0226)$ & $(0.005,0.3136,0.0512)$ & $(0.005,0.3136,0.0512)$ \\
\hline 30 & 0 & 1 & $(0.3309,0.1426,0.1196)$ & $(0.3899,0.243,0.1346)$ & $(0.3309,0.1426,0.1196)$ \\
\hline 35 & 0 & 1 & $(-0.0068,-0.101,0.0493)$ & $(-0.0004,-0.0326,0.061)$ & $(-0.0004,-0.0326,0.061)$ \\
\hline 40 & 0 & 1 & $(-0.071,-0.4388,0.041)$ & $(-0.1743,-0.4518,0.0499)$ & $(-0.071,-0.4388,0.041)$ \\
\hline 45 & 0 & 1 & $(0.0665,-0.114,-0.013)$ & $(0.1131,-0.1027,0.0013)$ & $(0.0665,-0.114,-0.013)$ \\
\hline 50 & 0 & 1 & $(-0.0797,0.0643,-0.0066)$ & $(0.0886,0.1865,0.0422)$ & $(-0.0797,0.0643,-0.0066)$ \\
\hline 54 & 0 & 1 & $(0.0162,0.2012,-0.0024)$ & $(0.009,0.2679,-0.0023)$ & $(0.0162,0.2012,-0.0024)$ \\
\hline \hline
\end{tabular}

Table B-6: Error for Localisation Symmetric Map (MCL) Case 1.6

\begin{tabular}{|c|c|c|c|c|c|}
\hline \hline$\#$ & $\mathrm{R}$ & $\mathrm{C}$ & Most Likely Cluster & Most Likely Sample & Closest Cluster \\
\hline \hline 1 & 0 & 4 & $(4.0993,-5.7515,-1.5826)$ & $(4.057,-5.774,-1.5885)$ & $(-0.1039,0.0585,-1.3405)$ \\
\hline 2 & 0 & 3 & $(-0.0647,0.0604,-0.0136)$ & $(-0.0529,0.0607,-0.0136)$ & $(-0.0529,0.0607,-0.0136)$ \\
\hline 3 & 0 & 1 & $(0.0097,0.07,-0.0048)$ & $(-0.0437,0.0689,-0.0048)$ & $(0.0097,0.07,-0.0048)$ \\
\hline 4 & 0 & 1 & $(0.0258,0.0706,0.0028)$ & $(0.104,0.0722,0.0028)$ & $(0.0258,0.0706,0.0028)$ \\
\hline 5 & 0 & 1 & $(0.0198,0.1004,0.0026)$ & $(0.0543,0.1011,0.0027)$ & $(0.0198,0.1004,0.0026)$ \\
\hline 10 & 0 & 1 & $(-0.1714,0.3404,-0.0016)$ & $(-0.1381,0.3371,-0.0092)$ & $(-0.1381,0.3371,-0.0092)$ \\
\hline 15 & 0 & 1 & $(-0.1256,0.2577,-0.0271)$ & $(-0.1453,0.2358,-0.0354)$ & $(-0.1453,0.2358,-0.0354)$ \\
\hline 20 & 0 & 1 & $(0.0201,0.426,-0.0038)$ & $(-0.0088,0.4226,0.0004)$ & $(-0.0088,0.4226,0.0004)$ \\
\hline 25 & 0 & 1 & $(-0.0425,0.0355,-0.0014)$ & $(-0.0279,0.0859,0.0013)$ & $(-0.0425,0.0355,-0.0014)$ \\
\hline 30 & 0 & 1 & $(0.2423,-0.0197,0.1237)$ & $(0.2767,-0.0008,0.1508)$ & $(0.2423,-0.0197,0.1237)$ \\
\hline 35 & 0 & 1 & $(0.0344,-0.0158,0.027)$ & $(0.0198,-0.0361,0.0301)$ & $(0.0344,-0.0158,0.027)$ \\
\hline 40 & 0 & 1 & $(-0.0576,-0.0981,0.0316)$ & $(-0.0537,-0.1474,0.0319)$ & $(-0.0576,-0.0981,0.0316)$ \\
\hline 45 & 0 & 1 & $(0.0438,0.0206,-0.0063)$ & $(0.0332,0.0287,0.0152)$ & $(0.0332,0.0287,0.0152)$ \\
\hline 50 & 0 & 1 & $(-0.014,0.0325,-0.0077)$ & $(0.0041,-0.0394,-0.0055)$ & $(-0.014,0.0325,-0.0077)$ \\
\hline 54 & 0 & 1 & $(0.0475,0.0847,-0.0102)$ & $(0.0491,0.1068,-0.0073)$ & $(0.0475,0.0847,-0.0102)$ \\
\hline \hline
\end{tabular}


Table B-7: Error for Localisation Symmetric Map (MCL) Case 1.7

\begin{tabular}{|c|c|c|c|c|c|}
\hline \hline$\#$ & $\mathrm{R}$ & $\mathrm{C}$ & Most Likely Cluster & Most Likely Sample & Closest Cluster \\
\hline \hline 1 & 0 & 4 & $(4.0819,-5.7103,-1.5821)$ & $(4.0573,-5.7766,-1.5831)$ & $(-0.1574,0.0473,-0.6852)$ \\
\hline 2 & 0 & 3 & $(-0.0316,0.0536,-0.0005)$ & $(-0.0542,0.0531,-0.0005)$ & $(-0.0316,0.0536,-0.0005)$ \\
\hline 3 & 0 & 1 & $(0.0026,0.0423,0.0239)$ & $(0.016,0.0426,0.0239)$ & $(0.0026,0.0423,0.0239)$ \\
\hline 4 & 0 & 1 & $(-0.1106,0.0059,0.0451)$ & $(-0.1116,0.0059,0.0451)$ & $(-0.1106,0.0059,0.0451)$ \\
\hline 5 & 0 & 1 & $(-0.0628,-0.028,0.0461)$ & $(-0.116,-0.0291,0.0461)$ & $(-0.0628,-0.028,0.0461)$ \\
\hline 10 & 0 & 1 & $(0.0551,-0.243,0.0234)$ & $(0.087,-0.2035,0.0089)$ & $(0.087,-0.2035,0.0089)$ \\
\hline 15 & 0 & 1 & $(0.0562,-0.1586,0.0134)$ & $(0.0533,-0.1494,0.0127)$ & $(0.0533,-0.1494,0.0127)$ \\
\hline 20 & 0 & 1 & $(-0.0544,-0.0629,-0.0135)$ & $(-0.0635,-0.058,-0.0073)$ & $(-0.0544,-0.0629,-0.0135)$ \\
\hline 25 & 0 & 1 & $(-0.0059,0.0208,-0.0058)$ & $(-0.0276,0.0084,-0.0071)$ & $(-0.0059,0.0208,-0.0058)$ \\
\hline 30 & 0 & 1 & $(0.265,-0.0978,0.1154)$ & $(0.2632,-0.1036,0.1115)$ & $(0.2632,-0.1036,0.1115)$ \\
\hline 35 & 0 & 1 & $(0.0797,-0.0122,0.009)$ & $(0.0842,-0.0268,0.0082)$ & $(0.0797,-0.0122,0.009)$ \\
\hline 40 & 0 & 1 & $(-0.0025,-0.0839,0.0297)$ & $(-0.0018,-0.1784,0.0298)$ & $(-0.0025,-0.0839,0.0297)$ \\
\hline 45 & 0 & 1 & $(-0.1367,-0.0084,0.0032)$ & $(-0.1369,-0.0301,0.0019)$ & $(-0.1367,-0.0084,0.0032)$ \\
\hline 50 & 0 & 1 & $(-0.0617,-0.0277,0.0071)$ & $(-0.0486,-0.0972,0.0065)$ & $(-0.0617,-0.0277,0.0071)$ \\
\hline 54 & 0 & 1 & $(-0.1452,0.0056,-0.0115)$ & $(-0.1589,0.0335,-0.0115)$ & $(-0.1452,0.0056,-0.0115)$ \\
\hline \hline
\end{tabular}

Table B-8: Error for Localisation Symmetric Map (MCL) Case 1.8

\begin{tabular}{|c|c|c|c|c|c|}
\hline \hline$\#$ & $\mathrm{R}$ & $\mathrm{C}$ & Most Likely Cluster & Most Likely Sample & Closest Cluster \\
\hline \hline 1 & 0 & 4 & $(4.0879,-5.7622,-1.5358)$ & $(4.9489,3.8364,-4.6805)$ & $(-0.0171,0.0301,-2.7631)$ \\
\hline 2 & 0 & 4 & $(3.2333,-4.5767,-1.6307)$ & $(3.2616,-4.6224,-1.6309)$ & $(-0.0842,-0.0035,-0.0178)$ \\
\hline 3 & 0 & 4 & $(0.022,0.0328,0.0192)$ & $(0.0007,0.0423,0.0239)$ & $(0.022,0.0328,0.0192)$ \\
\hline 4 & 0 & 3 & $(-0.0087,-0.0411,0.0008)$ & $(0.0076,-0.0808,-0.0321)$ & $(-0.0087,-0.0411,0.0008)$ \\
\hline 5 & 0 & 1 & $(0.0389,-0.03,0.0059)$ & $(0.0554,-0.0388,-0.0312)$ & $(0.0389,-0.03,0.0059)$ \\
\hline 10 & 0 & 1 & $(0.1314,-0.2366,0.0173)$ & $(0.129,-0.2795,0.0672)$ & $(0.1314,-0.2366,0.0173)$ \\
\hline 15 & 0 & 1 & $(-0.0117,-0.0907,0.0037)$ & $(0.1264,-0.1348,0.0271)$ & $(-0.0117,-0.0907,0.0037)$ \\
\hline 20 & 0 & 1 & $(-0.0734,-0.0135,-0.0103)$ & $(0.0096,0.0285,0.0132)$ & $(0.0096,0.0285,0.0132)$ \\
\hline 25 & 0 & 1 & $(-0.0056,0.1277,-0.0051)$ & $(0.0698,0.0933,0.0108)$ & $(0.0698,0.0933,0.0108)$ \\
\hline 30 & 0 & 1 & $(0.2708,-0.0293,0.0914)$ & $(0.3096,0.0275,0.1097)$ & $(0.2708,-0.0293,0.0914)$ \\
\hline 35 & 0 & 1 & $(0.016,-0.0484,0.0163)$ & $(0.062,-0.0941,0.0215)$ & $(0.016,-0.0484,0.0163)$ \\
\hline 40 & 0 & 1 & $(-0.0196,-0.2841,0.0282)$ & $(-0.0532,-0.1453,0.0369)$ & $(-0.0532,-0.1453,0.0369)$ \\
\hline 45 & 0 & 1 & $(-0.0935,-0.0624,-0.011)$ & $(-0.08,-0.1387,-0.0266)$ & $(-0.0935,-0.0624,-0.011)$ \\
\hline 50 & 0 & 1 & $(-0.0497,0.1343,0.0083)$ & $(-0.0258,0.1557,0.0001)$ & $(-0.0497,0.1343,0.0083)$ \\
\hline 54 & 0 & 1 & $(-0.1261,0.0674,-0.0106)$ & $(-0.0681,0.0834,-0.0149)$ & $(-0.0681,0.0834,-0.0149)$ \\
\hline \hline
\end{tabular}


Table B-9: Error for Localisation Symmetric Map (MCL) Case 1.9

\begin{tabular}{|c|c|c|c|c|c|}
\hline \hline$\#$ & $\mathrm{R}$ & $\mathrm{C}$ & Most Likely Cluster & Most Likely Sample & Closest Cluster \\
\hline \hline 1 & 0 & 4 & $(4.0654,-5.7406,-1.4931)$ & $(3.1142,-6.2366,-0.992)$ & $(0.0031,-0.0075,-3.1366)$ \\
\hline 2 & 0 & 4 & $(3.2552,-4.6215,-1.6356)$ & $(3.2968,-4.4962,-1.5544)$ & $(-0.0884,-0.109,-0.0421)$ \\
\hline 3. & 0 & 4 & $(0.034,-0.0318,-0.0061)$ & $(3.4304,2.3441,-4.6957)$ & $(0.034,-0.0318,-0.0061)$ \\
\hline 4 & 0 & 1 & $(0.1176,-0.1966,-0.1519)$ & $(-0.0219,-0.0837,-0.0321)$ & $(-0.0219,-0.0837,-0.0321)$ \\
\hline 5 & 0 & 1 & $(0.0796,-0.0473,-0.0368)$ & $(-0.0849,-0.0285,0.0461)$ & $(0.0796,-0.0473,-0.0368)$ \\
\hline 10 & 0 & 2 & $(0.1748,-0.0736,-0.0174)$ & $(0.1312,0.0595,-0.0788)$ & $(0.1312,0.0595,-0.0788)$ \\
\hline 15 & 0 & 1 & $(-0.0999,-0.0018,-0.0095)$ & $(0.0158,0.061,-0.0099)$ & $(0.0158,0.061,-0.0099)$ \\
\hline 20 & 0 & 1 & $(-0.1373,0.03,-0.0138)$ & $(-0.2169,-0.1186,-0.0195)$ & $(-0.1373,0.03,-0.0138)$ \\
\hline 25 & 0 & 1 & $(-0.0233,0.2039,0)$ & $(-0.0637,0.3195,-0.0097)$ & $(-0.0233,0.2039,0)$ \\
\hline 30 & 0 & 1 & $(0.2732,0.0057,0.0702)$ & $(0.2423,-0.1682,0.1039)$ & $(0.2732,0.0057,0.0702)$ \\
\hline 35 & 0 & 1 & $(-0.015,-0.0682,0.025)$ & $(-0.0299,0.1749,0.0079)$ & $(-0.015,-0.0682,0.025)$ \\
\hline 40 & 0 & 1 & $(-0.0253,-0.3966,0.0306)$ & $(-0.0433,-0.4379,0.0357)$ & $(-0.0253,-0.3966,0.0306)$ \\
\hline 45 & 0 & 1 & $(-0.0826,-0.1469,-0.0106)$ & $(-0.2,-0.0685,-0.0578)$ & $(-0.0826,-0.1469,-0.0106)$ \\
\hline 50 & 0 & 1 & $(-0.0571,0.159,0.0111)$ & $(-0.1541,0.1642,0.0245)$ & $(-0.0571,0.159,0.0111)$ \\
\hline 54 & 0 & 1 & $(-0.1391,0.1462,-0.0068)$ & $(-0.1328,0.2297,0.0088)$ & $(-0.1391,0.1462,-0.0068)$ \\
\hline \hline
\end{tabular}

Table B-10: Error for Localisation Symmetric Map (MCL) Case 2.1

\begin{tabular}{|c|c|c|c|c|c|}
\hline \hline$\#$ & $\mathrm{R}$ & $\mathrm{C}$ & Most Likely Cluster & Most Likely Sample & Closest Cluster \\
\hline \hline 1 & 0 & 4 & $(4.1042,-5.6548,-1.529)$ & $(0.2969,0.1252,-6.1851)$ & $(0.2969,0.1252,-6.1851)$ \\
\hline 2 & 0 & 2 & $(3.2854,-4.2879,-1.5741)$ & $(3.2851,-4.2291,-1.5741)$ & $(3.2851,-4.2291,-1.5741)$ \\
\hline 3 & 0 & 1 & $(2.3624,-3.0871,-1.5731)$ & $(2.3623,-3.0599,-1.5731)$ & $(2.3623,-3.0599,-1.5731)$ \\
\hline 4 & 0 & 1 & $(1.3949,-2.3656,-1.5722)$ & $(1.3916,-2.3671,-1.5722)$ & $(1.3916,-2.3671,-1.5722)$ \\
\hline 5 & 0 & 1 & $(0.4049,-1.254,-1.5721)$ & $(0.4055,-1.2606,-1.5725)$ & $(0.4049,-1.254,-1.5721)$ \\
\hline 10 & 1 & 1 & $(0.1037,-0.0132,0.0085)$ & $(0.1065,-0.0138,0.0091)$ & $(0.1037,-0.0132,0.0085)$ \\
\hline 15 & 1 & 1 & $(0.0037,-0.0346,0.0025)$ & $(0.0397,-0.0112,0.0104)$ & $(0.0037,-0.0346,0.0025)$ \\
\hline 20 & 1 & 1 & $(0.0144,0.088,0.0038)$ & $(0.0182,0.0909,0.0021)$ & $(0.0144,0.088,0.0038)$ \\
\hline 25 & 1 & 1 & $(0.018,0.0273,-0.0018)$ & $(0.0303,0.0392,0.0008)$ & $(0.018,0.0273,-0.0018)$ \\
\hline 30 & 1 & 1 & $(0.2784,-0.0848,0.1194)$ & $(0.2817,-0.0783,0.1399)$ & $(0.2784,-0.0848,0.1194)$ \\
\hline 35 & 1 & 1 & $(-0.0207,0.0195,0.0628)$ & $(-0.0209,0.0611,0.0628)$ & $(-0.0207,0.0195,0.0628)$ \\
\hline 40 & 1 & 1 & $(-0.3075,-0.0626,0.0631)$ & $(-0.3086,0.0486,0.0631)$ & $(-0.3086,0.0486,0.0631)$ \\
\hline 45 & 1 & 1 & $(-0.5374,-0.0059,0.0738)$ & $(-0.532,-0.0469,0.0912)$ & $(-0.532,-0.0469,0.0912)$ \\
\hline 50 & 1 & 1 & $(-0.3807,0.5679,0.0748)$ & $(-0.3841,0.5926,0.0748)$ & $(-0.3807,0.5679,0.0748)$ \\
\hline 54 & 1 & 1 & $(-0.1523,0.2282,0.0715)$ & $(-0.163,0.2617,0.0718)$ & $(-0.1523,0.2282,0.0715)$ \\
\hline \hline
\end{tabular}


Table B-11: Error for Localisation Symmetric Map (MCL) Case 2.2

\begin{tabular}{|c|c|c|c|c|c|}
\hline \hline$\#$ & $\mathrm{R}$ & $\mathrm{C}$ & Most Likely Cluster & Most Likely Sample & Closest Cluster \\
\hline \hline 1 & 0 & 4 & $(4.1098,-5.6539,-1.5375)$ & $(4.1525,-5.2536,-1.4979)$ & $(0.2554,0.0787,-5.632)$ \\
\hline 2 & 0 & 3 & $(3.2851,-4.7592,-1.661)$ & $(3.2858,-4.3932,-1.5741)$ & $(-0.0788,-0.326,-0.0633)$ \\
\hline 3 & 0 & 2 & $(2.363,-3.2228,-1.5731)$ & $(2.3633,-3.3159,-1.5731)$ & $(0.0473,-0.2602,-0.0624)$ \\
\hline 4 & 0 & 1 & $(0.1497,-0.168,-0.0614)$ & $(0.0966,-0.1727,-0.061)$ & $(0.0966,-0.1727,-0.061)$ \\
\hline 5 & 0 & 1 & $(0.216,-0.1017,-0.0613)$ & $(0.2009,-0.1025,-0.0609)$ & $(0.2009,-0.1025,-0.0609)$ \\
\hline 10 & 0 & 1 & $(-0.0758,0.3383,-0.0466)$ & $(-0.1409,0.3281,-0.0039)$ & $(-0.0758,0.3383,-0.0466)$ \\
\hline 15 & 0 & 1 & $(-0.2321,0.1891,-0.0511)$ & $(-0.2459,0.1555,-0.0596)$ & $(-0.2459,0.1555,-0.0596)$ \\
\hline 20 & 0 & 1 & $(0.029,0.3242,0.0237)$ & $(0.0981,0.3335,0.0154)$ & $(0.029,0.3242,0.0237)$ \\
\hline 25 & 0 & 1 & $(0.0978,0.1568,0.0083)$ & $(0.1218,0.1468,0.0233)$ & $(0.0978,0.1568,0.0083)$ \\
\hline 30 & 0 & 1 & $(0.3858,0.0136,0.122)$ & $(0.4182,-0.0109,0.1146)$ & $(0.3858,0.0136,0.122)$ \\
\hline 35 & 0 & 1 & $(0.129,-0.0373,0.0241)$ & $(0.1463,-0.1831,0.0219)$ & $(0.129,-0.0373,0.0241)$ \\
\hline 40 & 0 & 1 & $(0.0014,-0.2491,0.0272)$ & $(-0.06,-0.1168,0.0332)$ & $(-0.06,-0.1168,0.0332)$ \\
\hline 45 & 0 & 1 & $(-0.0811,-0.0957,0.0009)$ & $(-0.0207,-0.1356,-0.0557)$ & $(-0.0811,-0.0957,0.0009)$ \\
\hline 50 & 0 & 1 & $(-0.0687,0.1631,0.0093)$ & $(0.0163,0.1728,0.0082)$ & $(0.0163,0.1728,0.0082)$ \\
\hline 54 & 0 & 1 & $(-0.1222,0.058,0.0051)$ & $(-0.1195,0.0921,0.0052)$ & $(-0.1222,0.058,0.0051)$ \\
\hline \hline
\end{tabular}

Table B-12: Error for Localisation Symmetric Map (MCL) Case 2.3

\begin{tabular}{|c|c|c|c|c|c|}
\hline \hline$\#$ & $\mathrm{R}$ & $\mathrm{C}$ & Most Likely Cluster & Most Likely Sample & Closest Cluster \\
\hline \hline 1 & 0 & 4 & $(4.133,-5.7106,-1.5329)$ & $(4.1525,-5.2536,-1.4979)$ & $(0.1311,-0.0589,-3.9765)$ \\
\hline 2 & 0 & 3 & $(3.2927,-4.7636,-1.6579)$ & $(3.2975,-5.1202,-1.7328)$ & $(-0.0729,-0.3256,-0.0633)$ \\
\hline 3 & 0 & 3 & $(0.0586,-0.2594,-0.0624)$ & $(2.363,-3.2391,-1.5731)$ & $(0.0586,-0.2594,-0.0624)$ \\
\hline 4 & 0 & 2 & $(0.1254,-0.1704,-0.0614)$ & $(0.1444,-0.1682,-0.0622)$ & $(0.1254,-0.1704,-0.0614)$ \\
\hline 5 & 0 & 1 & $(0.2029,-0.1027,-0.0613)$ & $(0.2785,-0.0931,-0.0617)$ & $(0.2029,-0.1027,-0.0613)$ \\
\hline 10 & 0 & 1 & $(-0.0594,0.3495,-0.0709)$ & $(-0.0511,0.3446,-0.0239)$ & $(-0.0511,0.3446,-0.0239)$ \\
\hline 15 & 0 & 1 & $(-0.3236,0.1472,-0.0694)$ & $(-0.2532,0.1768,-0.0658)$ & $(-0.2532,0.1768,-0.0658)$ \\
\hline 20 & 0 & 1 & $(-0.0106,0.3219,0.02)$ & $(-0.0299,0.3685,0.0252)$ & $(-0.0106,0.3219,0.02)$ \\
\hline 25 & 0 & 1 & $(0.0688,0.379,0.014)$ & $(0.0756,0.365,0.0128)$ & $(0.0756,0.365,0.0128)$ \\
\hline 30 & 0 & 1 & $(0.2911,0.0614,0.0615)$ & $(0.3875,0.1245,0.1627)$ & $(0.2911,0.0614,0.0615)$ \\
\hline 35 & 0 & 1 & $(0.1129,-0.0908,0.0169)$ & $(0.2629,-0.11,-0.0286)$ & $(0.1129,-0.0908,0.0169)$ \\
\hline 40 & 0 & 1 & $(-0.0047,-0.3489,0.0232)$ & $(-0.0961,-0.1212,0.0405)$ & $(-0.0961,-0.1212,0.0405)$ \\
\hline 45 & 0 & 1 & $(-0.0368,-0.2357,-0.0025)$ & $(-0.0641,-0.346,0.0231)$ & $(-0.0368,-0.2357,-0.0025)$ \\
\hline 50 & 0 & 1 & $(-0.0473,0.1637,0.0077)$ & $(0.1129,0.0586,-0.0052)$ & $(0.1129,0.0586,-0.0052)$ \\
\hline 54 & 0 & 1 & $(-0.127,0.0805,0.0001)$ & $(-0.1357,0.0402,0.0004)$ & $(-0.1357,0.0402,0.0004)$ \\
\hline \hline
\end{tabular}


Table B-13: Error for Localisation Symmetric Map (MCL) Case 2.4

\begin{tabular}{|c|c|c|c|c|c|}
\hline \hline$\#$ & $\mathrm{R}$ & $\mathrm{C}$ & Most Likely Cluster & Most Likely Sample & Closest Cluster \\
\hline \hline 1 & 0 & 4 & $(4.1058,-5.6698,-1.535)$ & $(4.1527,-5.255,-1.4952)$ & $(0.2583,0.0804,-5.6654)$ \\
\hline 2 & 0 & 3 & $(3.2881,-4.786,-1.6616)$ & $(3.2828,-5.0127,-1.7263)$ & $(-0.0821,-0.3301,-0.0568)$ \\
\hline 3 & 0 & 2 & $(0.0506,-0.2738,-0.048)$ & $(0.0571,-0.2734,-0.048)$ & $(0.0506,-0.2738,-0.048)$ \\
\hline 4 & 0 & 1 & $(0.2535,-0.2206,-0.0404)$ & $(0.2128,-0.2232,-0.0404)$ & $(0.2128,-0.2232,-0.0404)$ \\
\hline 5 & 0 & 1 & $(0.2787,-0.1485,-0.0406)$ & $(0.3495,-0.1414,-0.0406)$ & $(0.2787,-0.1485,-0.0406)$ \\
\hline 10 & 0 & 1 & $(-0.0653,0.2553,-0.0414)$ & $(-0.0958,0.2459,-0.0416)$ & $(-0.0653,0.2553,-0.0414)$ \\
\hline 15 & 0 & 1 & $(-0.1968,0.0988,-0.0422)$ & $(-0.2145,0.1016,-0.0201)$ & $(-0.1968,0.0988,-0.0422)$ \\
\hline 20 & 0 & 1 & $(0.0005,0.3375,0.0046)$ & $(0.0691,0.3385,0.0176)$ & $(0.0005,0.3375,0.0046)$ \\
\hline 25 & 0 & 1 & $(-0.0136,0.231,0.0001)$ & $(-0.0566,0.1779,0.0297)$ & $(-0.0566,0.1779,0.0297)$ \\
\hline 30 & 0 & 1 & $(0.291,0.038,0.1221)$ & $(0.2906,0.0445,0.0931)$ & $(0.2906,0.0445,0.0931)$ \\
\hline 35 & 0 & 1 & $(0.0337,-0.0711,0.0293)$ & $(-0.0229,-0.2133,0.0282)$ & $(0.0337,-0.0711,0.0293)$ \\
\hline 40 & 0 & 1 & $(-0.0342,-0.306,0.0303)$ & $(-0.0541,-0.2186,0.0326)$ & $(-0.0541,-0.2186,0.0326)$ \\
\hline 45 & 0 & 1 & $(0.0873,-0.0301,-0.0018)$ & $(0.0998,0.0883,-0.0479)$ & $(0.0873,-0.0301,-0.0018)$ \\
\hline 50 & 0 & 1 & $(-0.0399,0.1066,-0.0113)$ & $(-0.0497,0.114,-0.0315)$ & $(-0.0399,0.1066,-0.0113)$ \\
\hline 54 & 0 & 1 & $(0.0416,0.1534,-0.0102)$ & $(0.0525,0.1511,-0.0062)$ & $(0.0416,0.1534,-0.0102)$ \\
\hline \hline
\end{tabular}

Table B-14: Error for Localisation Symmetric Map (MCL) Case 2.5

\begin{tabular}{|c|c|c|c|c|c|}
\hline \hline$\#$ & $\mathrm{R}$ & $\mathrm{C}$ & Most Likely Cluster & Most Likely Sample & Closest Cluster \\
\hline \hline 1 & 0 & 4 & $(4.1312,-5.7123,-1.531)$ & $(4.1527,-5.255,-1.4952)$ & $(0.1347,-0.0536,-4.0164)$ \\
\hline 2 & 0 & 3 & $(3.2975,-4.7814,-1.656)$ & $(3.3174,-5.2232,-1.7263)$ & $(-0.0633,-0.3289,-0.0568)$ \\
\hline 3 & 0 & 3 & $(0.0618,-0.2731,-0.048)$ & $(-0.0139,-0.278,-0.048)$ & $(-0.0139,-0.278,-0.048)$ \\
\hline 4 & 0 & 1 & $(0.4504,-0.5688,-0.2934)$ & $(0.2306,-0.2221,-0.0404)$ & $(0.2306,-0.2221,-0.0404)$ \\
\hline 5 & 0 & 1 & $(0.3055,-0.1599,-0.0584)$ & $(0.4446,-0.1352,-0.0406)$ & $(0.3055,-0.1599,-0.0584)$ \\
\hline 10 & 0 & 1 & $(-0.0547,0.2624,-0.0577)$ & $(-0.1968,0.2605,-0.0642)$ & $(-0.0547,0.2624,-0.0577)$ \\
\hline 15 & 0 & 1 & $(-0.2606,0.0725,-0.0518)$ & $(-0.1529,0.0988,-0.0508)$ & $(-0.1529,0.0988,-0.0508)$ \\
\hline 20 & 0 & 1 & $(-0.0986,0.3321,0.0325)$ & $(-0.1755,0.3471,0.0643)$ & $(-0.0986,0.3321,0.0325)$ \\
\hline 25 & 0 & 1 & $(0.0187,0.4364,0.0228)$ & $(0.1368,0.3947,0.0371)$ & $(0.1368,0.3947,0.0371)$ \\
\hline 30 & 0 & 1 & $(0.3371,0.159,0.1201)$ & $(0.3314,0.1142,0.0631)$ & $(0.3314,0.1142,0.0631)$ \\
\hline 35 & 0 & 1 & $(-0.0132,-0.103,0.0492)$ & $(-0.0601,-0.4339,0.0296)$ & $(-0.0132,-0.103,0.0492)$ \\
\hline 40 & 0 & 1 & $(-0.0781,-0.4388,0.0406)$ & $(-0.0007,-0.3073,0.0239)$ & $(-0.0007,-0.3073,0.0239)$ \\
\hline 45 & 0 & 1 & $(0.07,-0.13,-0.0051)$ & $(-0.1024,-0.1479,-0.0354)$ & $(0.07,-0.13,-0.0051)$ \\
\hline 50 & 0 & 1 & $(-0.0734,0.0573,-0.0077)$ & $(-0.0939,-0.082,-0.0304)$ & $(-0.0734,0.0573,-0.0077)$ \\
\hline 54 & 0 & 1 & $(0.0189,0.2079,-0.0023)$ & $(0.0318,0.1476,-0.0059)$ & $(0.0318,0.1476,-0.0059)$ \\
\hline \hline
\end{tabular}


Table B-15: Error for Localisation Symmetric Map (MCL) Case 2.6

\begin{tabular}{|c|c|c|c|c|c|}
\hline \hline$\#$ & $\mathrm{R}$ & $\mathrm{C}$ & Most Likely Cluster & Most Likely Sample & Closest Cluster \\
\hline \hline 1 & 0 & 4 & $(4.0828,-5.6923,-1.5165)$ & $(0.2971,0.1239,-6.1824)$ & $(0.2971,0.1239,-6.1824)$ \\
\hline 2 & 0 & 2 & $(3.2889,-4.2731,-1.5684)$ & $(3.2889,-4.2868,-1.5675)$ & $(3.2889,-4.2731,-1.5684)$ \\
\hline 3 & 0 & 1 & $(2.3649,-3.0994,-1.5588)$ & $(2.3648,-3.068,-1.5588)$ & $(2.3648,-3.068,-1.5588)$ \\
\hline 4 & 0 & 1 & $(1.4382,-2.1667,-1.5511)$ & $(1.4382,-2.1506,-1.5511)$ & $(1.4382,-2.1506,-1.5511)$ \\
\hline 5 & 0 & 1 & $(0.4908,-1.266,-1.5513)$ & $(0.4883,-1.2563,-1.5514)$ & $(0.4883,-1.2563,-1.5514)$ \\
\hline 10 & 1 & 1 & $(0.114,0.0025,-0.001)$ & $(0.1264,-0.0004,0.0013)$ & $(0.114,0.0025,-0.001)$ \\
\hline 15 & 1 & 1 & $(0.0102,-0.0519,0.0036)$ & $(-0.0334,-0.0708,0.0006)$ & $(0.0102,-0.0519,0.0036)$ \\
\hline 20 & 1 & 1 & $(0.0521,0.1413,-0.0088)$ & $(0.0585,0.1357,-0.0071)$ & $(0.0585,0.1357,-0.0071)$ \\
\hline 25 & 1 & 1 & $(-0.015,0.0114,-0.0136)$ & $(-0.0204,0.0271,-0.0191)$ & $(-0.015,0.0114,-0.0136)$ \\
\hline 30 & 1 & 1 & $(0.2575,-0.0499,0.1308)$ & $(0.2574,-0.0617,0.1311)$ & $(0.2575,-0.0499,0.1308)$ \\
\hline 35 & 1 & 1 & $(0.0292,-0.0175,0.0377)$ & $(0.0285,-0.0603,0.0377)$ & $(0.0292,-0.0175,0.0377)$ \\
\hline 40 & 1 & 1 & $(-0.1538,-0.1122,0.0467)$ & $(-0.1568,0.0475,0.0467)$ & $(-0.1568,0.0475,0.0467)$ \\
\hline 45 & 1 & 1 & $(-0.108,0.0249,0.0378)$ & $(-0.0928,0.0784,0.0332)$ & $(-0.108,0.0249,0.0378)$ \\
\hline 50 & 1 & 1 & $(-0.0355,0.1132,0.0398)$ & $(-0.0732,0.0008,0.0194)$ & $(-0.0732,0.0008,0.0194)$ \\
\hline 54 & 1 & 1 & $(0.0039,0.0693,0.0016)$ & $(0.0008,0.0542,0)$ & $(0.0008,0.0542,0)$ \\
\hline \hline
\end{tabular}

Table B-16: Error for Localisation Symmetric Map (MCL) Case 2.7

\begin{tabular}{|c|c|c|c|c|c|}
\hline \hline$\#$ & $\mathrm{R}$ & $\mathrm{C}$ & Most Likely Cluster & Most Likely Sample & Closest Cluster \\
\hline \hline 1 & 0 & 4 & $(0.2974,0.1212,-6.177)$ & $(0.2974,0.1212,-6.177)$ & $(0.2974,0.1212,-6.177)$ \\
\hline 2 & 0 & 1 & $(3.293,-4.8979,-1.6862)$ & $(3.2919,-5.0327,-1.7132)$ & $(3.293,-4.8979,-1.6862)$ \\
\hline 3 & 0 & 1 & $(2.3705,-3.1365,-1.5301)$ & $(2.3704,-3.1306,-1.5301)$ & $(2.3704,-3.1306,-1.5301)$ \\
\hline 4 & 0 & 1 & $(1.4362,-2.2247,-1.5089)$ & $(1.4361,-2.2039,-1.5089)$ & $(1.4361,-2.2039,-1.5089)$ \\
\hline 5 & 0 & 1 & $(0.5021,-1.4164,-1.5079)$ & $(0.5019,-1.3606,-1.5079)$ & $(0.5019,-1.3606,-1.5079)$ \\
\hline 10 & 1 & 1 & $(-5.7552,-1.9998,3.053)$ & $(-5.7552,-1.9998,3.053)$ & $(-5.7552,-1.9998,3.053)$ \\
\hline 15 & 1 & 1 & $(-2.385,-1.3196,3.1185)$ & $(-2.3809,-1.3228,3.121)$ & $(-2.385,-1.3196,3.1185)$ \\
\hline 20 & 1 & 1 & $(2.9993,-0.2313,-3.1367)$ & $(3.0328,-0.2165,-3.1415)$ & $(2.9993,-0.2313,-3.1367)$ \\
\hline 25 & 1 & 1 & $(2.4534,-9.2252,-3.1544)$ & $(2.473,-9.1572,-3.1544)$ & $(2.473,-9.1572,-3.1544)$ \\
\hline 30 & 1 & 1 & $(1.5643,-11.9709,3.2656)$ & $(1.5622,-11.9758,3.2669)$ & $(1.5643,-11.9709,3.2656)$ \\
\hline 35 & 1 & 1 & $(1.5985,-2.8792,3.1937)$ & $(1.6263,-2.8676,3.1958)$ & $(1.5985,-2.8792,3.1937)$ \\
\hline 40 & 1 & 1 & $(1.1099,6.3811,3.213)$ & $(1.1102,6.3353,3.213)$ & $(1.1102,6.3353,3.213)$ \\
\hline 45 & 1 & 1 & $(0.6713,12.1275,3.1825)$ & $(0.6646,12.15,3.2442)$ & $(0.6713,12.1275,3.1825)$ \\
\hline 50 & 1 & 1 & $(1.4309,6.3971,-3.0292)$ & $(1.4343,6.3781,-3.0299)$ & $(1.4343,6.3781,-3.0299)$ \\
\hline 54 & 1 & 1 & $(2.8877,-0.1205,-3.0609)$ & $(2.8877,-0.1205,-3.0609)$ & $(2.8877,-0.1205,-3.0609)$ \\
\hline \hline
\end{tabular}


Table B-17: Error for Localisation Symmetric Map (MCL) Case 2.8

\begin{tabular}{|c|c|c|c|c|c|}
\hline \hline$\#$ & $\mathrm{R}$ & $\mathrm{C}$ & Most Likely Cluster & Most Likely Sample & Closest Cluster \\
\hline \hline 1 & 0 & 4 & $(12.5445,0.7314,-2.8048)$ & $(14.4612,3.5067,-0.0727)$ & $(-2.1298,-5.4858,-3.6076)$ \\
\hline 2 & 0 & 2 & $(12.0765,0.7491,-2.8156)$ & $(12.9502,-7.7657,-5.1244)$ & $(-2.2963,7.5524,-2.2814)$ \\
\hline 3 & 0 & 2 & $(11.4682,1.7757,-2.4275)$ & $(10.1705,6.9645,-0.6231)$ & $(-3.3815,7.7643,-2.4116)$ \\
\hline 4 & 0 & 3 & $(11.0479,6.7982,-0.4888)$ & $(11.1473,8.0386,-0.6157)$ & $(-4.5389,8.3713,-2.413)$ \\
\hline 5 & 0 & 2 & $(10.363,7.6504,-0.5724)$ & $(10.1196,7.0647,-0.6189)$ & $(10.1196,7.0647,-0.6189)$ \\
\hline 10 & 1 & 4 & $(-0.1322,-0.0019,-0.0177)$ & $(-0.1687,-0.0494,-0.1006)$ & $(-0.1322,-0.0019,-0.0177)$ \\
\hline 15 & 1 & 1 & $(-0.1119,0.0973,-0.0211)$ & $(-0.1263,0.079,-0.0442)$ & $(-0.1119,0.0973,-0.0211)$ \\
\hline 20 & 1 & 1 & $(-0.0556,0.0473,-0.004)$ & $(0.1019,-0.014,-0.0247)$ & $(-0.0556,0.0473,-0.004)$ \\
\hline 25 & 1 & 1 & $(-0.0064,0.1308,-0.0065)$ & $(-0.0555,0.0049,0.0047)$ & $(-0.0555,0.0049,0.0047)$ \\
\hline 30 & 1 & 1 & $(0.2788,-0.0265,0.0933)$ & $(0.2806,0.0513,0.0677)$ & $(0.2806,0.0513,0.0677)$ \\
\hline 35 & 1 & 1 & $(0.02,-0.0497,0.0223)$ & $(0.0354,-0.1706,0.0196)$ & $(0.02,-0.0497,0.0223)$ \\
\hline 40 & 1 & 1 & $(-0.026,-0.2875,0.0332)$ & $(-0.0776,-0.185,0.0396)$ & $(-0.0776,-0.185,0.0396)$ \\
\hline 45 & 1 & 1 & $(-0.0911,-0.061,-0.0102)$ & $(-0.0347,0.0666,-0.0963)$ & $(-0.0911,-0.061,-0.0102)$ \\
\hline 50 & 1 & 1 & $(-0.0407,0.1272,0.0071)$ & $(-0.0474,0.3395,0.0069)$ & $(-0.0407,0.1272,0.0071)$ \\
\hline 54 & 1 & 1 & $(-0.125,0.0665,-0.012)$ & $(-0.1668,0.1664,-0.0166)$ & $(-0.125,0.0665,-0.012)$ \\
\hline \hline
\end{tabular}

Table B-18: Error for Localisation Symmetric Map (MCL) Case 2.9

\begin{tabular}{|c|c|c|c|c|c|}
\hline \hline$\#$ & $\mathrm{R}$ & $\mathrm{C}$ & Most Likely Cluster & Most Likely Sample & Closest Cluster \\
\hline \hline 1 & 0 & 4 & $(4.1269,-5.7191,-1.5262)$ & $(4.153,-5.2577,-1.4898)$ & $(0.1384,-0.0533,-4.057)$ \\
\hline 2 & 0 & 3 & $(3.3034,-4.8124,-1.65)$ & $(-0.2295,-0.3475,-0.0437)$ & $(-0.053,-0.3361,-0.0437)$ \\
\hline 3 & 0 & 3 & $(0.0592,-0.301,-0.0193)$ & $(-0.0429,-0.3076,-0.0193)$ & $(0.0592,-0.301,-0.0193)$ \\
\hline 4 & 0 & 2 & $(-0.0438,-0.3014,0.0019)$ & $(0.0027,-0.2984,0.0019)$ & $(0.0027,-0.2984,0.0019)$ \\
\hline 5 & 0 & 1 & $(-0.002,-0.2935,0.0027)$ & $(0.1241,-0.2852,0.0029)$ & $(-0.002,-0.2935,0.0027)$ \\
\hline 10 & 0 & 1 & $(0.1907,-0.3275,-0.0109)$ & $(0.3525,-0.2921,0.0041)$ & $(0.1907,-0.3275,-0.0109)$ \\
\hline 15 & 0 & 1 & $(-0.006,-0.2831,0.0165)$ & $(-0.0608,-0.2404,0.0271)$ & $(-0.0608,-0.2404,0.0271)$ \\
\hline 20 & 0 & 1 & $(-0.1512,-0.1863,-0.0237)$ & $(-0.1407,-0.1698,-0.0132)$ & $(-0.1407,-0.1698,-0.0132)$ \\
\hline 25 & 0 & 1 & $(-0.0249,0.1938,-0.001)$ & $(-0.0644,0.3362,-0.0209)$ & $(-0.0249,0.1938,-0.001)$ \\
\hline 30 & 0 & 1 & $(0.2752,-0.0042,0.0697)$ & $(0.2371,-0.1276,-0.0422)$ & $(0.2371,-0.1276,-0.0422)$ \\
\hline 35 & 0 & 1 & $(-0.0202,-0.0671,0.0272)$ & $(0.0536,0.0433,-0.0031)$ & $(0.0536,0.0433,-0.0031)$ \\
\hline 40 & 0 & 1 & $(-0.0443,-0.4059,0.0323)$ & $(-0.0414,-0.3631,0.0242)$ & $(-0.0414,-0.3631,0.0242)$ \\
\hline 45 & 0 & 1 & $(-0.1001,-0.1481,-0.009)$ & $(-0.1146,-0.1774,-0.0019)$ & $(-0.1001,-0.1481,-0.009)$ \\
\hline 50 & 0 & 1 & $(-0.0671,0.1609,0.0126)$ & $(-0.067,0.112,0.0162)$ & $(-0.067,0.112,0.0162)$ \\
\hline 54 & 0 & 1 & $(-0.1446,0.1528,-0.0053)$ & $(-0.2148,0.1658,-0.0044)$ & $(-0.1446,0.1528,-0.0053)$ \\
\hline \hline
\end{tabular}


Table B-19: Error for Localisation Symmetric Map (MCL) Case 3.1

\begin{tabular}{|c|c|c|c|c|c|}
\hline \hline$\#$ & $\mathrm{R}$ & $\mathrm{C}$ & Most Likely Cluster & Most Likely Sample & Closest Cluster \\
\hline \hline 1 & 0 & 1 & $(5.7559,5.1022,1.6835)$ & $(5.7559,5.1022,1.6835)$ & $(5.7559,5.1022,1.6835)$ \\
\hline 2 & 0 & 1 & $(4.6929,3.7986,-4.5988)$ & $(4.7042,3.8982,-4.5988)$ & $(4.6929,3.7986,-4.5988)$ \\
\hline 3 & 0 & 1 & $(3.6237,2.467,1.684)$ & $(3.6236,2.4661,1.684)$ & $(3.6236,2.4661,1.684)$ \\
\hline 4 & 0 & 1 & $(2.6315,1.0977,-4.5987)$ & $(2.6315,1.0977,-4.5987)$ & $(2.6315,1.0977,-4.5987)$ \\
\hline 5 & 0 & 1 & $(1.6658,0.1064,-4.5979)$ & $(1.6669,0.1266,-4.5979)$ & $(1.6658,0.1064,-4.5979)$ \\
\hline 10 & 1 & 1 & $(-0.4112,0.0366,-0.0094)$ & $(-0.4112,0.0366,-0.0094)$ & $(-0.4112,0.0366,-0.0094)$ \\
\hline 15 & 2 & 1 & $(-1.6287,1.6342,-1.705)$ & $(-1.6287,1.6342,-1.705)$ & $(-1.6287,1.6342,-1.705)$ \\
\hline 20 & 3 & 1 & $(1.6923,0.7936,1.4846)$ & $(1.6923,0.7936,1.4871)$ & $(1.6923,0.7936,1.4846)$ \\
\hline 25 & 3 & 1 & $(5.036,-3.7838,1.4728)$ & $(5.036,-3.7838,1.4728)$ & $(5.036,-3.7838,1.4728)$ \\
\hline 30 & 3 & 1 & $(6.0002,-5.5245,1.8024)$ & $(6.0001,-5.5237,1.8101)$ & $(6.0002,-5.5245,1.8024)$ \\
\hline 35 & 4 & 1 & $(2.1885,-0.0085,1.5824)$ & $(2.1885,-0.0085,1.5824)$ & $(2.1885,-0.0085,1.5824)$ \\
\hline 40 & 5 & 1 & $(2.2342,3.6064,-1.293)$ & $(0.7939,2.5775,-16.3413)$ & $(2.2342,3.6064,-1.293)$ \\
\hline 45 & 5 & 1 & $(-4.92,6.5346,1.7221)$ & $(-4.9516,6.5357,1.7215)$ & $(-4.92,6.5346,1.7221)$ \\
\hline 50 & 6 & 1 & $(2.7001,3.93,-3.9625)$ & $(4.2625,-3.6602,-12.4153)$ & $(2.7001,3.93,-3.9625)$ \\
\hline 54 & 6 & 1 & $(1.607,1.3986,1.3929)$ & $(1.6208,1.4036,1.3942)$ & $(1.607,1.3986,1.3929)$ \\
\hline
\end{tabular}

Table B-20: Error for Localisation Symmetric Map (MCL) Case 3.2

\begin{tabular}{|c|c|c|c|c|c|}
\hline \hline$\#$ & $\mathrm{R}$ & $\mathrm{C}$ & Most Likely Cluster & Most Likely Sample & Closest Cluster \\
\hline \hline 1 & 0 & 1 & $(4.3678,-6.0699,-1.7333)$ & $(4.3678,-6.0699,-1.7333)$ & $(4.3678,-6.0699,-1.7333)$ \\
\hline 2 & 0 & 1 & $(3.2852,-5.0451,-1.7328)$ & $(3.2563,-4.8693,-1.7328)$ & $(3.2563,-4.8693,-1.7328)$ \\
\hline 3 & 0 & 1 & $(2.2146,-4.118,-1.7319)$ & $(2.2064,-4.0677,-1.7319)$ & $(2.2064,-4.0677,-1.7319)$ \\
\hline 4 & 0 & 1 & $(1.066,-3.1044,-1.7309)$ & $(1.0642,-3.0871,-1.7309)$ & $(1.0642,-3.0871,-1.7309)$ \\
\hline 5 & 0 & 1 & $(-0.1518,-1.82,-1.731)$ & $(-0.1518,-1.82,-1.731)$ & $(-0.1518,-1.82,-1.731)$ \\
\hline 10 & 0 & 1 & $(-5.2552,1.1451,-1.731)$ & $(-5.2535,1.1493,-1.7164)$ & $(-5.2535,1.1493,-1.7164)$ \\
\hline 15 & 1 & 1 & $(-0.9194,-2.0167,1.6578)$ & $(-0.9192,-2.0182,1.6578)$ & $(-0.9194,-2.0167,1.6578)$ \\
\hline 20 & 1 & 1 & $(1.5358,1.8192,1.5603)$ & $(1.5491,1.792,1.5606)$ & $(1.5491,1.792,1.5606)$ \\
\hline 25 & 1 & 1 & $(5.4345,-3.131,-4.6972)$ & $(5.3726,-3.1216,-4.6977)$ & $(5.3726,-3.1216,-4.6977)$ \\
\hline 30 & 1 & 1 & $(6.1923,-5.0954,1.5605)$ & $(6.1565,-5.094,1.5359)$ & $(6.1565,-5.094,1.5359)$ \\
\hline 35 & 1 & 1 & $(1.9364,-0.847,1.5602)$ & $(1.8819,-0.8528,1.5593)$ & $(1.8819,-0.8528,1.5593)$ \\
\hline 40 & 1 & 1 & $(-2.8833,3.3527,1.5597)$ & $(-2.8689,3.3539,1.5597)$ & $(-2.8689,3.3539,1.5597)$ \\
\hline 45 & 1 & 1 & $(-5.383,6.1311,1.5655)$ & $(-5.3598,6.1323,1.5639)$ & $(-5.3598,6.1323,1.5639)$ \\
\hline 50 & 1 & 1 & $(-2.2598,4.0148,1.5706)$ & $(-2.2207,4.0086,1.5764)$ & $(-2.2207,4.0086,1.5764)$ \\
\hline 54 & 1 & 1 & $(1.404,1.6123,1.5608)$ & $(1.3284,1.5818,1.5593)$ & $(1.3284,1.5818,1.5593)$ \\
\hline \hline
\end{tabular}


Table B-21: Error for Localisation Symmetric Map (MCL) Case 3.3

\begin{tabular}{|c|c|c|c|c|c|}
\hline \hline$\#$ & $\mathrm{R}$ & $\mathrm{C}$ & Most Likely Cluster & Most Likely Sample & Closest Cluster \\
\hline \hline 1 & 0 & 2 & $(4.4155,-6.0042,-1.7301)$ & $(4.3678,-6.0699,-1.7333)$ & $(4.4155,-6.0042,-1.7301)$ \\
\hline 2 & 0 & 1 & $(3.3036,-5.034,-1.7318)$ & $(3.2814,-5.0218,-1.7328)$ & $(3.2814,-5.0218,-1.7328)$ \\
\hline 3 & 0 & 1 & $(2.6698,-3.4785,-1.7043)$ & $(2.7076,-3.7556,-1.7043)$ & $(2.6698,-3.4785,-1.7043)$ \\
\hline 4 & 0 & 1 & $(1.524,-2.3139,-1.7034)$ & $(1.5246,-2.3012,-1.7028)$ & $(1.5246,-2.3012,-1.7028)$ \\
\hline 5 & 0 & 1 & $(0.3906,-1.2545,-1.7033)$ & $(0.406,-1.3357,-1.7035)$ & $(0.3906,-1.2545,-1.7033)$ \\
\hline 10 & 0 & 1 & $(-4.5897,1.5998,-1.6817)$ & $(-4.5886,1.5748,-1.6866)$ & $(-4.5886,1.5748,-1.6866)$ \\
\hline 15 & 0 & 1 & $(-1.9818,-0.1558,-1.7489)$ & $(-2.0076,-0.0721,-1.7538)$ & $(-1.9818,-0.1558,-1.7489)$ \\
\hline 20 & 0 & 1 & $(1.3177,-2.1567,-1.5753)$ & $(1.3266,-2.2128,-1.568)$ & $(1.3177,-2.1567,-1.5753)$ \\
\hline 25 & 0 & 1 & $(-3.2914,-6.1878,-1.5779)$ & $(-3.2173,-6.1964,-1.5794)$ & $(-3.2173,-6.1964,-1.5794)$ \\
\hline 30 & 0 & 1 & $(-4.5508,-7.0012,4.6462)$ & $(-4.5647,-6.9834,4.6341)$ & $(-4.5647,-6.9834,4.6341)$ \\
\hline 35 & 0 & 1 & $(-0.6852,-2.0457,4.695)$ & $(-0.6732,-2.0464,4.6953)$ & $(-0.6732,-2.0464,4.6953)$ \\
\hline 40 & 0 & 1 & $(3.5969,2.9421,4.696)$ & $(3.7079,2.9552,4.6957)$ & $(3.5969,2.9421,4.696)$ \\
\hline 45 & 0 & 1 & $(5.6922,5.9763,-1.5609)$ & $(5.6732,5.9782,-1.5869)$ & $(5.6732,5.9782,-1.5869)$ \\
\hline 50 & 0 & 1 & $(3.6209,2.7135,-1.5709)$ & $(3.6093,2.6969,-1.5748)$ & $(3.6093,2.6969,-1.5748)$ \\
\hline 54 & 0 & 1 & $(1.5158,-1.3201,-1.5754)$ & $(1.5163,-1.3246,-1.5789)$ & $(1.5158,-1.3201,-1.5754)$ \\
\hline \hline
\end{tabular}

Table B-22: Error for Localisation Symmetric Map (MCL) Case 3.4

\begin{tabular}{|c|c|c|c|c|c|}
\hline \hline$\#$ & $\mathrm{R}$ & $\mathrm{C}$ & Most Likely Cluster & Most Likely Sample & Closest Cluster \\
\hline \hline 1 & 0 & 1 & $(4.368,-6.0713,-1.7306)$ & $(4.368,-6.0713,-1.7306)$ & $(4.368,-6.0713,-1.7306)$ \\
\hline 2 & 0 & 1 & $(3.2878,-5.0431,-1.7263)$ & $(3.2598,-4.8731,-1.7263)$ & $(3.2598,-4.8731,-1.7263)$ \\
\hline 3 & 0 & 1 & $(2.2142,-4.1137,-1.7175)$ & $(2.221,-4.1551,-1.7175)$ & $(2.2142,-4.1137,-1.7175)$ \\
\hline 4 & 0 & 1 & $(1.0951,-2.9381,-1.7099)$ & $(1.1019,-2.9792,-1.7099)$ & $(1.0951,-2.9381,-1.7099)$ \\
\hline 5 & 0 & 1 & $(-0.072,-1.681,-1.7101)$ & $(-0.0714,-1.6768,-1.7102)$ & $(-0.0714,-1.6768,-1.7102)$ \\
\hline 10 & 0 & 1 & $(-5.1721,1.2437,-1.7164)$ & $(-5.1636,1.2387,-1.7049)$ & $(-5.1636,1.2387,-1.7049)$ \\
\hline 15 & 1 & 1 & $(-0.5245,-1.7676,1.4994)$ & $(-0.5137,-1.7593,1.5053)$ & $(-0.5137,-1.7593,1.5053)$ \\
\hline 20 & 1 & 1 & $(1.7792,1.6419,1.1179)$ & $(1.7836,1.6227,1.6452)$ & $(1.7792,1.6419,1.1179)$ \\
\hline 25 & 1 & 1 & $(5.3442,-3.5799,-4.6642)$ & $(5.3614,-3.5736,-4.6585)$ & $(5.3442,-3.5799,-4.6642)$ \\
\hline 30 & 1 & 1 & $(6.1851,-5.4815,1.7524)$ & $(6.1999,-5.5148,1.7287)$ & $(6.1851,-5.4815,1.7524)$ \\
\hline 35 & 1 & 1 & $(1.5834,-0.3004,1.7219)$ & $(1.5231,-0.2927,1.7219)$ & $(1.5231,-0.2927,1.7219)$ \\
\hline 40 & 1 & 1 & $(-2.9297,4.5776,1.7308)$ & $(-2.8577,4.5729,1.7308)$ & $(-2.8577,4.5729,1.7308)$ \\
\hline 45 & 1 & 1 & $(-5.6163,7.2455,-4.3562)$ & $(-5.6402,7.24,-4.3706)$ & $(-5.6163,7.2455,-4.3562)$ \\
\hline 50 & 1 & 1 & $(-1.355,4.4087,-4.3758)$ & $(-1.339,4.4174,-4.3779)$ & $(-1.355,4.4087,-4.3758)$ \\
\hline 54 & 1 & 1 & $(2.4965,0.872,-4.3864)$ & $(2.4963,0.872,-4.3864)$ & $(2.4963,0.872,-4.3864)$ \\
\hline \hline
\end{tabular}


Table B-23: Error for Localisation Symmetric Map (MCL) Case 3.5

\begin{tabular}{|c|c|c|c|c|c|}
\hline \hline$\#$ & $\mathrm{R}$ & $\mathrm{C}$ & Most Likely Cluster & Most Likely Sample & Closest Cluster \\
\hline \hline 1 & 0 & 2 & $(12.5138,0.3746,-2.8928)$ & $(13.9502,-4.6935,-5.4734)$ & $(-1.321,6.8944,-2.3258)$ \\
\hline 2 & 0 & 2 & $(12.3358,0.0701,-3.2806)$ & $(-3.0779,7.3546,-2.4786)$ & $(-2.8353,7.4559,-2.2435)$ \\
\hline 3 & 0 & 4 & $(11.774,6.7065,-0.5504)$ & $(12.6182,-5.615,-6.192)$ & $(-4.0627,6.259,-2.4)$ \\
\hline 4 & 0 & 3 & $(11.038,-6.4512,-5.7281)$ & $(10.5951,-7.8937,-5.4934)$ & $(8.4514,8.3852,-0.6451)$ \\
\hline 5 & 0 & 3 & $(10.3734,-8.2712,-5.579)$ & $(10.2468,-8.4036,-5.4934)$ & $(10.8857,7.4318,-0.1753)$ \\
\hline 10 & 1 & 2 & $(-1.8662,-4.2179,-4.7737)$ & $(-1.8485,-4.0598,-4.7644)$ & $(-3.5277,1.8333,-1.4315)$ \\
\hline 15 & 1 & 1 & $(-0.7481,-1.6753,1.5081)$ & $(-0.6428,-1.8934,1.5201)$ & $(-0.7481,-1.6753,1.5081)$ \\
\hline 20 & 1 & 1 & $(1.6992,1.6669,0.4236)$ & $(1.6276,1.6914,1.5982)$ & $(1.6992,1.6669,0.4236)$ \\
\hline 25 & 1 & 1 & $(5.5565,-3.6716,-4.6551)$ & $(5.6121,-3.6611,-4.6774)$ & $(5.5565,-3.6716,-4.6551)$ \\
\hline 30 & 1 & 1 & $(6.4556,-5.568,1.7584)$ & $(6.4304,-5.4678,1.7591)$ & $(6.4304,-5.4678,1.7591)$ \\
\hline 35 & 1 & 1 & $(1.726,-0.9989,1.5615)$ & $(1.8125,-0.9961,1.5615)$ & $(1.726,-0.9989,1.5615)$ \\
\hline 40 & 1 & 1 & $(-3.0222,3.1937,1.5706)$ & $(-3.2448,3.1721,1.5705)$ & $(-3.0222,3.1937,1.5706)$ \\
\hline 45 & 1 & 1 & $(-5.7318,5.4695,-4.7757)$ & $(-5.735,5.475,-4.7747)$ & $(-5.7318,5.4695,-4.7757)$ \\
\hline 50 & 1 & 1 & $(-2.4122,3.8373,1.48)$ & $(-2.4036,3.8726,1.4674)$ & $(-2.4122,3.8373,1.48)$ \\
\hline 54 & 1 & 1 & $(1.5352,1.4323,1.5099)$ & $(1.3615,1.3964,1.506)$ & $(1.3615,1.3964,1.506)$ \\
\hline
\end{tabular}

Table B-24: Error for Localisation Symmetric Map (MCL) Case 3.6

\begin{tabular}{|c|c|c|c|c|c|}
\hline \hline$\#$ & $\mathrm{R}$ & $\mathrm{C}$ & Most Likely Cluster & Most Likely Sample & Closest Cluster \\
\hline \hline 1 & 0 & 1 & $(4.368,-6.0713,-1.7306)$ & $(4.368,-6.0713,-1.7306)$ & $(4.368,-6.0713,-1.7306)$ \\
\hline 2 & 0 & 1 & $(3.2759,-4.9709,-1.7263)$ & $(3.2598,-4.8731,-1.7263)$ & $(3.2598,-4.8731,-1.7263)$ \\
\hline 3 & 0 & 1 & $(2.192,-3.9783,-1.7175)$ & $(2.1613,-3.7919,-1.7175)$ & $(2.1613,-3.7919,-1.7175)$ \\
\hline 4 & 1 & 1 & $(3.9424,0.1242,-6.3959)$ & $(-3.1989,-5.856,17.1749)$ & $(3.9424,0.1242,-6.3959)$ \\
\hline 5 & 1 & 1 & $(0.5599,-0.6248,-1.0341)$ & $(0.8085,-0.6842,-2.77)$ & $(0.5599,-0.6248,-1.0341)$ \\
\hline 10 & 2 & 2 & $(0.6506,0.1723,-5.9182)$ & $(0.6579,0.1718,-5.9059)$ & $(0.6579,0.1718,-5.9059)$ \\
\hline 15 & 4 & 1 & $(0.3761,0.232,-4.4564)$ & $(-7.147,-5.4733,22.2342)$ & $(0.3761,0.232,-4.4564)$ \\
\hline 20 & 4 & 1 & $(0.161,0.5404,-0.0273)$ & $(0.161,0.5405,-0.024)$ & $(0.161,0.5405,-0.024)$ \\
\hline 25 & 4 & 1 & $(-0.0379,-0.0129,-0.0369)$ & $(-0.0416,-0.0451,-0.0368)$ & $(-0.0379,-0.0129,-0.0369)$ \\
\hline 30 & 4 & 1 & $(0.2404,-0.0602,0.1169)$ & $(0.2173,-0.0921,0.1178)$ & $(0.2173,-0.0921,0.1178)$ \\
\hline 35 & 4 & 1 & $(0.017,-0.0191,0.0296)$ & $(0.0225,-0.0444,0.0282)$ & $(0.017,-0.0191,0.0296)$ \\
\hline 40 & 4 & 1 & $(-0.1166,-0.1032,0.0373)$ & $(-0.1249,-0.0816,0.038)$ & $(-0.1249,-0.0816,0.038)$ \\
\hline 45 & 4 & 1 & $(-0.0294,0.0294,0.016)$ & $(-0.0477,0.0725,0.0109)$ & $(-0.0294,0.0294,0.016)$ \\
\hline 50 & 4 & 1 & $(-0.0307,0.039,0.0096)$ & $(-0.0303,-0.0921,-0.0039)$ & $(-0.0307,0.039,0.0096)$ \\
\hline 54 & 4 & 1 & $(0.0285,0.0718,-0.0056)$ & $(0.025,0.0671,-0.0053)$ & $(0.025,0.0671,-0.0053)$ \\
\hline \hline
\end{tabular}


Table B-25: Error for Localisation Symmetric Map (MCL) Case 3.7

\begin{tabular}{|c|c|c|c|c|c|}
\hline \hline$\#$ & $\mathrm{R}$ & $\mathrm{C}$ & Most Likely Cluster & Most Likely Sample & Closest Cluster \\
\hline \hline 1 & 0 & 1 & $(4.3683,-6.0739,-1.7252)$ & $(4.3683,-6.0739,-1.7252)$ & $(4.3683,-6.0739,-1.7252)$ \\
\hline 2 & 0 & 1 & $(3.2771,-4.9424,-1.7132)$ & $(3.2669,-4.8804,-1.7132)$ & $(3.2669,-4.8804,-1.7132)$ \\
\hline 3 & 0 & 1 & $(2.1916,-3.9699,-1.6888)$ & $(2.1668,-3.8193,-1.6888)$ & $(2.1668,-3.8193,-1.6888)$ \\
\hline 4 & 1 & 1 & $(3.9404,0.0624,-6.3536)$ & $(-3.2009,-5.9179,17.2171)$ & $(3.9404,0.0624,-6.3536)$ \\
\hline 5 & 1 & 1 & $(0.4513,-0.7264,-0.1587)$ & $(0.4513,-0.7264,-0.1587)$ & $(0.4513,-0.7264,-0.1587)$ \\
\hline 10 & 2 & 1 & $(-1.916,-5.4142,1.8772)$ & $(-1.916,-5.4142,1.8772)$ & $(-1.916,-5.4142,1.8772)$ \\
\hline 15 & 3 & 1 & $(0.0141,-0.5931,0.1091)$ & $(0.0203,-0.5928,0.1091)$ & $(0.0203,-0.5928,0.1091)$ \\
\hline 20 & 3 & 1 & $(-0.2479,-0.2939,-0.0324)$ & $(-0.2479,-0.2928,-0.0331)$ & $(-0.2479,-0.2928,-0.0331)$ \\
\hline 25 & 3 & 1 & $(-0.1374,0.0588,0.0045)$ & $(-0.1363,0.0731,0.0045)$ & $(-0.1374,0.0588,0.0045)$ \\
\hline 30 & 3 & 1 & $(0.206,-0.0724,0.0897)$ & $(0.1967,-0.0815,0.065)$ & $(0.1967,-0.0815,0.065)$ \\
\hline 35 & 3 & 1 & $(-0.0182,-0.0091,0.0248)$ & $(-0.0138,0.0216,0.0238)$ & $(-0.0182,-0.0091,0.0248)$ \\
\hline 40 & 3 & 1 & $(-0.1701,-0.1132,0.0453)$ & $(-0.1728,-0.0574,0.0454)$ & $(-0.1728,-0.0574,0.0454)$ \\
\hline 45 & 3 & 1 & $(-0.3491,-0.0466,0.0209)$ & $(-0.3354,-0.0679,-0.0322)$ & $(-0.3354,-0.0679,-0.0322)$ \\
\hline 50 & 3 & 1 & $(-0.2366,0.014,0.0296)$ & $(-0.2304,-0.027,0.0296)$ & $(-0.2304,-0.027,0.0296)$ \\
\hline 54 & 3 & 1 & $(-0.2724,0.0113,0.0089)$ & $(-0.2689,0.0087,0.0079)$ & $(-0.2689,0.0087,0.0079)$ \\
\hline \hline
\end{tabular}

Table B-26: Error for Localisation Symmetric Map (MCL) Case 3.8

\begin{tabular}{|c|c|c|c|c|c|}
\hline \hline$\#$ & $\mathrm{R}$ & $\mathrm{C}$ & Most Likely Cluster & Most Likely Sample & Closest Cluster \\
\hline \hline 1 & 0 & 1 & $(4.3683,-6.0739,-1.7252)$ & $(4.3683,-6.0739,-1.7252)$ & $(4.3683,-6.0739,-1.7252)$ \\
\hline 2 & 0 & 1 & $(3.2932,-5.0407,-1.7132)$ & $(3.2669,-4.8804,-1.7132)$ & $(3.2669,-4.8804,-1.7132)$ \\
\hline 3 & 0 & 1 & $(2.2153,-4.1143,-1.6888)$ & $(2.2334,-4.2242,-1.6888)$ & $(2.2153,-4.1143,-1.6888)$ \\
\hline 4 & 0 & 1 & $(1.0891,-2.9759,-1.6676)$ & $(1.0975,-3.0266,-1.6676)$ & $(1.0891,-2.9759,-1.6676)$ \\
\hline 5 & 0 & 1 & $(-0.0486,-1.7692,-1.6666)$ & $(-0.046,-1.785,-1.6666)$ & $(-0.0486,-1.7692,-1.6666)$ \\
\hline 10 & 0 & 1 & $(-4.5426,1.6055,4.7127)$ & $(-4.5346,1.6169,4.726)$ & $(-4.5426,1.6055,4.7127)$ \\
\hline 15 & 0 & 1 & $(-2.226,0.1402,-1.6683)$ & $(-2.2476,0.1875,-1.6617)$ & $(-2.226,0.1402,-1.6683)$ \\
\hline 20 & 0 & 1 & $(1.2783,-2.2319,-1.5536)$ & $(1.2557,-2.1616,-1.5467)$ & $(1.2557,-2.1616,-1.5467)$ \\
\hline 25 & 0 & 1 & $(-2.935,-6.3388,-1.5616)$ & $(-2.8638,-6.3448,-1.5616)$ & $(-2.8638,-6.3448,-1.5616)$ \\
\hline 30 & 0 & 1 & $(-4.2806,-7.162,4.6124)$ & $(-4.2352,-7.1756,4.646)$ & $(-4.2806,-7.162,4.6124)$ \\
\hline 35 & 0 & 1 & $(-0.1468,-1.9404,4.5833)$ & $(-0.0873,-1.9325,4.5826)$ & $(-0.0873,-1.9325,4.5826)$ \\
\hline 40 & 0 & 1 & $(3.7,3.4796,4.607)$ & $(3.8741,3.5142,4.607)$ & $(3.7,3.4796,4.607)$ \\
\hline 45 & 0 & 1 & $(5.5897,6.8206,-1.6576)$ & $(5.59,6.828,-1.6933)$ & $(5.5897,6.8206,-1.6576)$ \\
\hline 50 & 0 & 1 & $(4.0923,2.9994,-1.8858)$ & $(4.0991,3.003,-1.8859)$ & $(4.0923,2.9994,-1.8858)$ \\
\hline 54 & 0 & 1 & $(1.3815,-2.6209,-1.9036)$ & $(1.4054,-2.6259,-1.9026)$ & $(1.3815,-2.6209,-1.9036)$ \\
\hline \hline
\end{tabular}


Table B-27: Error for Localisation Symmetric Map (MCL) Case 3.9

\begin{tabular}{|c|c|c|c|c|c|}
\hline \hline$\#$ & $\mathrm{R}$ & $\mathrm{C}$ & Most Likely Cluster & Most Likely Sample & Closest Cluster \\
\hline \hline 1 & 0 & 2 & $(4.4113,-6.0146,-1.7223)$ & $(4.3683,-6.0739,-1.7252)$ & $(4.4113,-6.0146,-1.7223)$ \\
\hline 2 & 0 & 1 & $(3.3127,-5.0397,-1.7121)$ & $(3.289,-5.0149,-1.7132)$ & $(3.289,-5.0149,-1.7132)$ \\
\hline 3 & 0 & 1 & $(2.6762,-3.5077,-1.6612)$ & $(2.6722,-3.4778,-1.6612)$ & $(2.6722,-3.4778,-1.6612)$ \\
\hline 4 & 0 & 1 & $(1.592,-2.4303,-1.64)$ & $(1.5768,-2.3186,-1.64)$ & $(1.5768,-2.3186,-1.64)$ \\
\hline 5 & 0 & 1 & $(0.5125,-1.3898,-1.6391)$ & $(0.5145,-1.4045,-1.6391)$ & $(0.5125,-1.3898,-1.6391)$ \\
\hline 10 & 0 & 1 & $(-4.0043,2.3903,4.6567)$ & $(-3.9715,2.3469,4.6974)$ & $(-3.9715,2.3469,4.6974)$ \\
\hline 15 & 0 & 1 & $(-1.8715,0.6352,-1.5509)$ & $(-1.8834,0.6798,-1.5496)$ & $(-1.8715,0.6352,-1.5509)$ \\
\hline 20 & 0 & 1 & $(1.4083,-1.9526,-1.5548)$ & $(1.4122,-2.0185,-1.5669)$ & $(1.4083,-1.9526,-1.5548)$ \\
\hline 25 & 0 & 1 & $(-3.2514,-6.0187,-1.5445)$ & $(-3.4115,-5.9361,-1.5427)$ & $(-3.2514,-6.0187,-1.5445)$ \\
\hline 30 & 0 & 1 & $(-4.5667,-6.8069,3.8138)$ & $(-4.5439,-6.7737,4.6706)$ & $(-4.5667,-6.8069,3.8138)$ \\
\hline 35 & 0 & 1 & $(-0.2597,-1.9212,4.6399)$ & $(-0.1479,-2.0516,4.653)$ & $(-0.2597,-1.9212,4.6399)$ \\
\hline 40 & 0 & 1 & $(3.7259,3.3238,4.6594)$ & $(3.9465,3.357,4.6593)$ & $(3.7259,3.3238,4.6594)$ \\
\hline 45 & 0 & 1 & $(5.5789,6.5494,-1.6359)$ & $(5.6093,6.5722,-1.7197)$ & $(5.5789,6.5494,-1.6359)$ \\
\hline 50 & 0 & 1 & $(3.7032,2.9373,-1.7482)$ & $(3.7323,2.9416,-1.7472)$ & $(3.7032,2.9373,-1.7482)$ \\
\hline 54 & 0 & 1 & $(1.3215,-1.8326,-1.7555)$ & $(1.2737,-1.912,-1.7625)$ & $(1.3215,-1.8326,-1.7555)$ \\
\hline \hline
\end{tabular}




\section{B.1.2 CPF-MCL}

Table B-28: Error for Localisation Symmetric Map (CPF-MCL) Case 1

\begin{tabular}{|c|c|c|c|c|}
\hline \hline$\#$ & $\mathrm{R}$ & $\mathrm{C}$ & Most Likely Sample & Closest Cluster \\
\hline \hline 5 & 0 & 4 & $(-0.0061,0.1267,0.01)$ & $(-0.0061,0.1267,0.01)$ \\
\hline 10 & 0 & 2 & $(-5.9825,-1.9283,-3.1447)$ & $(-0.0974,0.3194,0.0045)$ \\
\hline 15 & 0 & 2 & $(-2.4091,-1.2225,3.1436)$ & $(-0.0942,0.2592,-0.0247)$ \\
\hline 20 & 0 & 2 & $(3.0867,-0.1641,-3.1387)$ & $(0.048,0.3875,0.0349)$ \\
\hline 25 & 0 & 5 & $(4.7961,-3.4163,-4.7393)$ & $(0.1346,0.0525,0.0213)$ \\
\hline 30 & 0 & 4 & $(1.5874,-11.9146,3.2684)$ & $(0.4918,-0.0648,0.2016)$ \\
\hline 35 & 0 & 3 & $(1.276,-2.8921,3.2233)$ & $(-0.2654,-0.3281,0.1574)$ \\
\hline 40 & 0 & 2 & $(0.9838,6.3125,3.2232)$ & $(-0.9934,-0.3978,0.1578)$ \\
\hline 45 & 0 & 2 & $(0.8861,12.0975,3.2576)$ & $(-1.4545,0.0737,0.1667)$ \\
\hline 50 & 0 & 3 & $(-0.0509,0.0259,0.0061)$ & $(-0.0509,0.0259,0.0061)$ \\
\hline 54 & 0 & 2 & $(-0.1132,0.0212,0.0061)$ & $(-0.1132,0.0212,0.0061)$ \\
\hline \hline
\end{tabular}

Table B-29: Error for Localisation Symmetric Map (CPF-MCL) Case 2

\begin{tabular}{|c|c|c|c|c|}
\hline \hline$\#$ & $\mathrm{R}$ & $\mathrm{C}$ & Most Likely Sample & Closest Cluster \\
\hline \hline 5 & 0 & 5 & $(0.1036,0.1504,-0.0181)$ & $(0.1036,0.1504,-0.0181)$ \\
\hline 10 & 0 & 3 & $(-5.9351,-2.3294,-3.1888)$ & $(-0.2193,0.4274,-0.0279)$ \\
\hline 15 & 0 & 4 & $(-1.4865,-1.7074,1.573)$ & $(-0.2886,0.2834,-0.0653)$ \\
\hline 20 & 0 & 4 & $(3.0314,-0.4055,-3.1205)$ & $(-0.0073,0.5201,0.0481)$ \\
\hline 25 & 0 & 5 & $(2.337,-9.4523,-3.1241)$ & $(0.1675,0.269,0.0453)$ \\
\hline 30 & 0 & 4 & $(1.4871,-11.9872,3.2642)$ & $(0.5204,0.0212,0.1647)$ \\
\hline 35 & 0 & 4 & $(1.0784,-2.8762,3.1963)$ & $(0.0209,-0.0348,0.1021)$ \\
\hline 40 & 0 & 4 & $(0.6046,6.5146,3.192)$ & $(-0.1537,-0.2497,0.0754)$ \\
\hline 45 & 0 & 4 & $(0.3824,12.1655,3.1675)$ & $(-0.4162,-0.0881,0.0413)$ \\
\hline 50 & 0 & 4 & $(1.5208,6.466,-3.109)$ & $(-0.2955,0.2906,0.0499)$ \\
\hline 54 & 0 & 4 & $(3.039,0.185,-3.1153)$ & $(-0.2108,0.1344,0.0458)$ \\
\hline \hline
\end{tabular}


Table B-30: Error for Localisation Symmetric Map (CPF-MCL) Case 3

\begin{tabular}{|c|c|c|c|c|}
\hline \hline$\#$ & $\mathrm{R}$ & $\mathrm{C}$ & Most Likely Sample & Closest Cluster \\
\hline \hline 5 & 0 & 5 & $(0.1242,0.1258,-0.0255)$ & $(0.1242,0.1258,-0.0255)$ \\
\hline 10 & 0 & 4 & $(-5.9617,-2.3266,-3.2041)$ & $(0.0463,0.1607,0.0179)$ \\
\hline 15 & 0 & 5 & $(-0.2066,0.4115,-0.0602)$ & $(-0.2074,0.0133,-0.0444)$ \\
\hline 20 & 0 & 4 & $(3.1426,-0.1201,-3.1328)$ & $(-0.0091,0.19,0.0093)$ \\
\hline 25 & 0 & 5 & $(2.4434,-9.4678,-3.1325)$ & $(0.0207,0.2928,0.0023)$ \\
\hline 30 & 0 & 4 & $(1.6511,-12.0426,3.1833)$ & $(0.261,0.0573,0.0473)$ \\
\hline 35 & 0 & 4 & $(0.131,-0.0873,0.0142)$ & $(0.131,-0.0873,0.0142)$ \\
\hline 40 & 0 & 5 & $(0.522,6.6293,3.1637)$ & $(0.0083,-0.3538,0.0213)$ \\
\hline 45 & 0 & 5 & $(0.1917,12.3081,3.143)$ & $(-0.0414,-0.23,-0.0032)$ \\
\hline 50 & 0 & 4 & $(1.4018,6.5545,-3.129)$ & $(-0.0726,0.1623,0.0129)$ \\
\hline 54 & 0 & 4 & $(2.9953,0.1578,-3.1368)$ & $(-0.1257,0.0918,0.0063)$ \\
\hline \hline
\end{tabular}

Table B-31: Error for Localisation Symmetric Map (CPF-MCL) Case 4

\begin{tabular}{|c|c|c|c|c|}
\hline \hline$\#$ & $\mathrm{R}$ & $\mathrm{C}$ & Most Likely Sample & Closest Cluster \\
\hline \hline 5 & 0 & 5 & $(0.1063,0.1042,0.0026)$ & $(0.1063,0.1042,0.0026)$ \\
\hline 10 & 0 & 4 & $(-5.9311,-2.2343,-3.1804)$ & $(-0.1651,0.355,-0.0298)$ \\
\hline 15 & 0 & 4 & $(-0.8509,-1.5347,1.5157)$ & $(-0.2448,0.1866,-0.0519)$ \\
\hline 20 & 0 & 4 & $(-0.0337,0.4236,0.0134)$ & $(-0.0337,0.4236,0.0134)$ \\
\hline 25 & 0 & 5 & $(-0.0213,0.2865,0.0134)$ & $(-0.0213,0.2865,0.0134)$ \\
\hline 30 & 0 & 4 & $(5.8698,-5.2699,1.6378)$ & $(0.2709,0.0252,0.1138)$ \\
\hline 35 & 0 & 4 & $(-0.0226,-0.0884,0.0438)$ & $(-0.0226,-0.0884,0.0438)$ \\
\hline 40 & 0 & 5 & $(-0.0108,-0.3068,0.0297)$ & $(-0.0108,-0.3068,0.0297)$ \\
\hline 45 & 0 & 5 & $(-0.1064,12.0117,-3.1299)$ & $(0.1016,-0.0199,-0.0015)$ \\
\hline 50 & 0 & 4 & $(-0.0233,0.1157,-0.0162)$ & $(-0.0233,0.1157,-0.0162)$ \\
\hline 54 & 0 & 4 & $(0.0539,0.1606,-0.0122)$ & $(0.0539,0.1606,-0.0122)$ \\
\hline \hline
\end{tabular}


Table B-32: Error for Localisation Symmetric Map (CPF-MCL) Case 5

\begin{tabular}{|c|c|c|c|c|}
\hline \hline$\#$ & $\mathrm{R}$ & $\mathrm{C}$ & Most Likely Sample & Closest Cluster \\
\hline \hline 5 & 0 & 5 & $(0.1343,0.1045,0.0024)$ & $(0.1343,0.1045,0.0024)$ \\
\hline 10 & 0 & 4 & $(-5.952,-2.241,-3.1959)$ & $(-0.2031,0.3575,-0.035)$ \\
\hline 15 & 0 & 4 & $(-2.2395,-1.166,3.0985)$ & $(-0.3474,0.1507,-0.0697)$ \\
\hline 20 & 0 & 4 & $(3.1785,-0.4211,-3.1101)$ & $(-0.1048,0.3769,0.0335)$ \\
\hline 25 & 0 & 5 & $(2.0388,-9.5704,-3.1178)$ & $(0.0198,0.479,0.0215)$ \\
\hline 30 & 0 & 4 & $(0.0452,-0.2025,-0.0182)$ & $(0.0452,-0.2025,-0.0182)$ \\
\hline 35 & 0 & 4 & $(-0.0359,-0.173,-0.0441)$ & $(-0.0359,-0.173,-0.0441)$ \\
\hline 40 & 0 & 4 & $(0.9178,6.7339,3.1889)$ & $(0.0031,-0.329,-0.0123)$ \\
\hline 45 & 0 & 4 & $(-0.0864,12.1049,-3.1156)$ & $(0.2322,-0.1081,-0.0243)$ \\
\hline 50 & 0 & 4 & $(-0.0073,0.0539,-0.0345)$ & $(-0.0073,0.0539,-0.0345)$ \\
\hline 54 & 0 & 4 & $(0.0775,0.2259,-0.0203)$ & $(0.0775,0.2259,-0.0203)$ \\
\hline \hline
\end{tabular}

Table B-33: Error for Localisation Symmetric Map (CPF-MCL) Case 6

\begin{tabular}{|c|c|c|c|c|}
\hline \hline$\#$ & $\mathrm{R}$ & $\mathrm{C}$ & Most Likely Sample & Closest Cluster \\
\hline \hline 5 & 0 & 4 & $(0.0195,0.1004,0.0027)$ & $(0.0195,0.1004,0.0027)$ \\
\hline 10 & 0 & 2 & $(-6.0062,-1.8342,-3.1479)$ & $(-0.1742,0.3311,0.0053)$ \\
\hline 15 & 0 & 2 & $(-2.482,-0.9379,3.1523)$ & $(-0.1215,0.2636,-0.0264)$ \\
\hline 20 & 0 & 2 & $(3.0688,-0.1709,-3.1448)$ & $(0.0926,0.4084,-0.016)$ \\
\hline 25 & 0 & 5 & $(-2.5145,-5.6579,-1.5084)$ & $(-0.0201,0.0111,-0.0181)$ \\
\hline 30 & 0 & 4 & $(5.8707,-5.2749,1.6687)$ & $(0.2716,-0.0421,0.137)$ \\
\hline 35 & 0 & 3 & $(-0.0959,-0.0307,0.066)$ & $(-0.0959,-0.0307,0.066)$ \\
\hline 40 & 0 & 2 & $(-0.4084,-0.1078,0.0749)$ & $(-0.4084,-0.1078,0.0749)$ \\
\hline 45 & 0 & 2 & $(-0.4819,-0.0709,-0.5742)$ & $(-0.4819,-0.0709,-0.5742)$ \\
\hline 50 & 0 & 2 & $(3.85,2.6813,-1.5393)$ & $(0.1114,0.3478,-0.0554)$ \\
\hline 54 & 0 & 2 & $(0.1206,-0.058,-0.0615)$ & $(0.1206,-0.058,-0.0615)$ \\
\hline \hline
\end{tabular}


Table B-34: Error for Localisation Symmetric Map (CPF-MCL) Case 7

\begin{tabular}{|c|c|c|c|c|}
\hline \hline$\#$ & $\mathrm{R}$ & $\mathrm{C}$ & Most Likely Sample & Closest Cluster \\
\hline \hline 5 & 0 & 3 & $(-0.0649,-0.0281,0.0461)$ & $(-0.0649,-0.0281,0.0461)$ \\
\hline 10 & 0 & 1 & $(0.0554,-0.2455,0.0236)$ & $(0.0554,-0.2455,0.0236)$ \\
\hline 15 & 0 & 3 & $(0.0595,-0.1711,0.014)$ & $(0.0595,-0.1711,0.014)$ \\
\hline 20 & 0 & 3 & $(-0.0615,-0.0686,-0.0146)$ & $(-0.0615,-0.0686,-0.0146)$ \\
\hline 25 & 0 & 6 & $(5.0044,-3.4157,-4.6632)$ & $(-0.0024,0.0285,-0.0025)$ \\
\hline 30 & 0 & 4 & $(-4.0057,-6.7749,3.8983)$ & $(0.2983,-0.0796,0.1279)$ \\
\hline 35 & 0 & 3 & $(1.6607,-2.8659,3.2162)$ & $(-0.2028,-0.0596,0.0878)$ \\
\hline 40 & 0 & 2 & $(1.3129,6.3407,3.2375)$ & $(-0.6465,-0.0047,0.1089)$ \\
\hline 45 & 0 & 2 & $(0.9725,12.1517,3.224)$ & $(-1.0174,-0.0977,0.1319)$ \\
\hline 50 & 0 & 1 & $(2.4868,4.0101,-4.5413)$ & $(2.4868,4.0101,-4.5413)$ \\
\hline 54 & 0 & 2 & $(-0.0934,0.0084,-0.0014)$ & $(-0.0934,0.0084,-0.0014)$ \\
\hline \hline
\end{tabular}

Table B-35: Error for Localisation Symmetric Map (CPF-MCL) Case 8

\begin{tabular}{|c|c|c|c|c|}
\hline \hline$\#$ & $\mathrm{R}$ & $\mathrm{C}$ & Most Likely Sample & Closest Cluster \\
\hline \hline 5 & 0 & 5 & $(0.0288,-0.0304,0.0079)$ & $(0.0288,-0.0304,0.0079)$ \\
\hline 10 & 0 & 5 & $(0.1244,-0.2392,0.0179)$ & $(0.1244,-0.2392,0.0179)$ \\
\hline 15 & 0 & 5 & $(-1.4966,0.0721,-1.6923)$ & $(0.0438,-0.1743,0.0185)$ \\
\hline 20 & 0 & 4 & $(-0.0987,-0.0854,-0.0179)$ & $(-0.0987,-0.0854,-0.0179)$ \\
\hline 25 & 0 & 5 & $(-0.0054,0.1469,-0.0036)$ & $(-0.0054,0.1469,-0.0036)$ \\
\hline 30 & 0 & 4 & $(-4.2358,-6.7097,3.1635)$ & $(0.2626,-0.0147,0.0895)$ \\
\hline 35 & 0 & 4 & $(1.4885,-2.858,3.1649)$ & $(0.0314,-0.0383,0.0185)$ \\
\hline 40 & 0 & 5 & $(0.8218,6.5618,3.1771)$ & $(-0.0056,-0.281,0.0302)$ \\
\hline 45 & 0 & 4 & $(0.2046,12.1687,3.1347)$ & $(-0.1153,-0.0711,-0.0078)$ \\
\hline 50 & 0 & 4 & $(-0.0674,0.129,0.0117)$ & $(-0.0674,0.129,0.0117)$ \\
\hline 54 & 0 & 4 & $(-0.1347,0.0656,-0.0078)$ & $(-0.1347,0.0656,-0.0078)$ \\
\hline \hline
\end{tabular}


Table B-36: Error for Localisation Symmetric Map (CPF-MCL) Case 9

\begin{tabular}{|c|c|c|c|c|}
\hline \hline$\#$ & $\mathrm{R}$ & $\mathrm{C}$ & Most Likely Sample & Closest Cluster \\
\hline \hline 5 & 0 & 5 & $(0.0664,-0.0406,-0.0141)$ & $(0.0664,-0.0406,-0.0141)$ \\
\hline 10 & 0 & 4 & $(0.1724,-0.0931,-0.013)$ & $(0.1724,-0.0931,-0.013)$ \\
\hline 15 & 0 & 5 & $(-2.3077,-1.4224,3.1121)$ & $(-0.1275,-0.0137,-0.0167)$ \\
\hline 20 & 0 & 4 & $(-0.1307,0.0327,-0.0151)$ & $(-0.1307,0.0327,-0.0151)$ \\
\hline 25 & 0 & 5 & $(2.4416,-9.4374,-3.1406)$ & $(-0.0784,0.2361,-0.0157)$ \\
\hline 30 & 0 & 4 & $(0.2512,0.0101,0.0601)$ & $(0.2512,0.0101,0.0601)$ \\
\hline 35 & 0 & 4 & $(-0.0123,-0.0771,0.0202)$ & $(-0.0123,-0.0771,0.0202)$ \\
\hline 40 & 0 & 4 & $(0.8028,6.6979,3.1717)$ & $(0.0019,-0.4047,0.0212)$ \\
\hline 45 & 0 & 4 & $(0.1802,12.2568,3.125)$ & $(-0.0145,-0.154,-0.0157)$ \\
\hline 50 & 0 & 4 & $(1.2353,6.5633,-3.1333)$ & $(-0.0294,0.1624,0.0067)$ \\
\hline 54 & 0 & 4 & $(-0.1318,0.1484,-0.0108)$ & $(-0.1318,0.1484,-0.0108)$ \\
\hline \hline
\end{tabular}




\section{B.1.3 UCPF-MCL}

Table B-37: Error for Localisation Symmetric Map (UCPF-MCL) Case 1

\begin{tabular}{|c|c|c|c|c|}
\hline \hline$\#$ & $\mathrm{R}$ & $\mathrm{C}$ & Most Likely Sample & Closest Cluster \\
\hline \hline 1 & 0 & 9 & $(4.1303,-6.648,-1.5602)$ & $(-0.5477,-0.1144,-0.058)$ \\
\hline 2 & 0 & 6 & $(-0.2889,-0.1618,-0.0663)$ & $(-0.2889,-0.1618,-0.0663)$ \\
\hline 3 & 0 & 6 & $(0.0129,0.0172,-2.5734)$ & $(0.0345,-0.0657,-0.0582)$ \\
\hline 4 & 0 & 5 & $(3.5348,-1.2547,-3.1986)$ & $(0.0802,0.1313,-0.0303)$ \\
\hline 5 & 0 & 4 & $(0.0978,0.0991,-0.0266)$ & $(0.0978,0.0991,-0.0266)$ \\
\hline 10 & 0 & 2 & $(-0.1492,0.2863,-0.0173)$ & $(-0.1492,0.2863,-0.0173)$ \\
\hline 15 & 0 & 2 & $(-0.0719,0.2234,-0.0174)$ & $(-0.0719,0.2234,-0.0174)$ \\
\hline 20 & 0 & 2 & $(3.0276,-0.2714,-3.1281)$ & $(0.2811,0.2483,-0.2936)$ \\
\hline 25 & 0 & 2 & $(2.2983,-9.221,-3.1124)$ & $(0.0351,-0.017,-0.0073)$ \\
\hline 30 & 0 & 2 & $(0.267,-0.1261,0.0912)$ & $(0.267,-0.1261,0.0912)$ \\
\hline 35 & 0 & 2 & $(0.0674,-0.0123,0.0215)$ & $(0.0674,-0.0123,0.0215)$ \\
\hline 40 & 0 & 2 & $(-0.0312,-0.0861,0.0225)$ & $(-0.0312,-0.0861,0.0225)$ \\
\hline 45 & 0 & 2 & $(0.4023,12.0572,3.1294)$ & $(-0.1313,0.0146,-0.0399)$ \\
\hline 50 & 0 & 2 & $(-0.0927,-0.2039,-0.0208)$ & $(-0.0927,-0.2039,-0.0208)$ \\
\hline 54 & 0 & 2 & $(-0.163,0.0552,0.0095)$ & $(-0.163,0.0552,0.0095)$ \\
\hline \hline
\end{tabular}

Table B-38: Error for Localisation Symmetric Map (UCPF-MCL) Case 2

\begin{tabular}{|c|c|c|c|c|}
\hline \hline$\#$ & $\mathrm{R}$ & $\mathrm{C}$ & Most Likely Sample & Closest Cluster \\
\hline \hline 1 & 0 & 9 & $(4.1271,-5.6875,-1.5269)$ & $(-0.5802,0.0793,-0.0456)$ \\
\hline 2 & 0 & 6 & $(-0.233,0.0894,-0.0385)$ & $(-0.233,0.0894,-0.0385)$ \\
\hline 3 & 0 & 6 & $(5.462,-1.0229,-3.171)$ & $(0.0624,0.1408,-0.032)$ \\
\hline 4 & 0 & 5 & $(3.5983,-1.1452,-3.1775)$ & $(0.0852,0.2052,-0.0368)$ \\
\hline 5 & 0 & 5 & $(1.7553,-1.2897,-3.1751)$ & $(0.0372,0.1935,-0.0234)$ \\
\hline 10 & 0 & 2 & $(-5.7743,-2.3308,-3.1609)$ & $(-0.2136,0.3273,-0.0204)$ \\
\hline 15 & 0 & 2 & $(-2.3297,-1.5487,3.1247)$ & $(-0.0565,0.0609,-0.0115)$ \\
\hline 20 & 0 & 2 & $(0.049,0.1712,0.0102)$ & $(0.049,0.1712,0.0102)$ \\
\hline 25 & 0 & 2 & $(0.0243,0.0329,0.0002)$ & $(0.0243,0.0329,0.0002)$ \\
\hline 30 & 0 & 2 & $(0.2581,-0.0866,0.0788)$ & $(0.2581,-0.0866,0.0788)$ \\
\hline 35 & 0 & 2 & $(1.1177,-2.9677,3.1648)$ & $(0.0587,0.0766,0.0199)$ \\
\hline 40 & 0 & 2 & $(-0.0271,-0.0845,0.0211)$ & $(-0.0271,-0.0845,0.0211)$ \\
\hline 45 & 0 & 2 & $(0.2686,12.1131,3.115)$ & $(-0.1279,-0.0348,-0.0322)$ \\
\hline 50 & 0 & 2 & $(-0.1169,-0.1427,-0.0218)$ & $(-0.1169,-0.1427,-0.0218)$ \\
\hline 54 & 0 & 2 & $(3.0349,0.2167,-3.1306)$ & $(-0.1625,0.0459,0.0108)$ \\
\hline \hline
\end{tabular}


Table B-39: Error for Localisation Symmetric Map (UCPF-MCL) Case 3

\begin{tabular}{|c|c|c|c|c|}
\hline \hline$\#$ & $\mathrm{R}$ & $\mathrm{C}$ & Most Likely Sample & Closest Cluster \\
\hline \hline 1 & 0 & 8 & $(4.0736,-6.5091,-1.4961)$ & $(-0.0662,-0.1115,-0.045)$ \\
\hline 2 & 0 & 7 & $(2.9618,-6.9817,-1.5448)$ & $(-1.5833,-0.0055,-0.0966)$ \\
\hline 3 & 0 & 7 & $(5.3653,-1.3957,-3.1672)$ & $(-2.2064,-0.8059,-0.2248)$ \\
\hline 4 & 0 & 7 & $(3.1965,1.2705,-4.7961)$ & $(-0.0172,-0.5292,-0.0141)$ \\
\hline 5 & 0 & 7 & $(1.9232,-1.3231,-3.1333)$ & $(0.0058,-0.1984,-0.0014)$ \\
\hline 10 & 0 & 2 & $(-0.1537,0.1492,-0.0468)$ & $(-0.1537,0.1492,-0.0468)$ \\
\hline 15 & 0 & 2 & $(-2.4175,-1.5191,3.1535)$ & $(-0.0187,0.138,0.0052)$ \\
\hline 20 & 0 & 2 & $(0.2092,0.2185,0.0975)$ & $(0.2092,0.2185,0.0975)$ \\
\hline 25 & 0 & 1 & $(0.0836,-0.2399,0.0982)$ & $(0.0836,-0.2399,0.0982)$ \\
\hline 30 & 0 & 1 & $(0.3464,-0.1259,-0.3621)$ & $(0.3464,-0.1259,-0.3621)$ \\
\hline 35 & 0 & 1 & $(0.0906,-0.253,-0.0114)$ & $(0.0906,-0.253,-0.0114)$ \\
\hline 40 & 0 & 1 & $(-0.0671,-0.551,-0.0554)$ & $(-0.0671,-0.551,-0.0554)$ \\
\hline 45 & 0 & 1 & $(0.1682,-0.1606,-0.0274)$ & $(0.1682,-0.1606,-0.0274)$ \\
\hline 50 & 0 & 1 & $(-0.036,0.1445,-0.0052)$ & $(-0.036,0.1445,-0.0052)$ \\
\hline 54 & 0 & 1 & $(-0.0884,0.0364,0.0142)$ & $(-0.0884,0.0364,0.0142)$ \\
\hline \hline
\end{tabular}

Table B-40: Error for Localisation Symmetric Map (UCPF-MCL) Case 4

\begin{tabular}{|c|c|c|c|c|}
\hline \hline$\#$ & $\mathrm{R}$ & $\mathrm{C}$ & Most Likely Sample & Closest Cluster \\
\hline \hline 1 & 0 & 9 & $(4.1357,-6.6441,-1.5569)$ & $(-0.5801,0.0779,-0.0429)$ \\
\hline 2 & 0 & 7 & $(4.1684,3.2092,-4.6568)$ & $(-0.3484,0.2227,-0.1087)$ \\
\hline 3 & 0 & 6 & $(0.0045,0.2392,-6.2339)$ & $(0.0517,0.1938,-1.5061)$ \\
\hline 4 & 0 & 6 & $(0.1269,0.1835,-0.8052)$ & $(0.1269,0.1835,-0.8052)$ \\
\hline 5 & 0 & 5 & $(0.0596,0.2022,0.0008)$ & $(0.0596,0.2022,0.0008)$ \\
\hline 10 & 0 & 2 & $(-5.8247,-2.2083,-3.152)$ & $(-0.1907,0.2656,-0.0118)$ \\
\hline 15 & 0 & 2 & $(-2.3393,-1.1747,3.1101)$ & $(-0.1415,0.1225,-0.0359)$ \\
\hline 20 & 0 & 2 & $(2.9331,-0.294,-3.1684)$ & $(0.2365,0.2767,-0.0421)$ \\
\hline 25 & 0 & 2 & $(2.1098,-9.1016,-3.1806)$ & $(-0.057,-0.0717,-0.0568)$ \\
\hline 30 & 0 & 2 & $(0.1668,-0.0811,0.0985)$ & $(0.1668,-0.0811,0.0985)$ \\
\hline 35 & 0 & 2 & $(1.5565,-2.8344,3.1592)$ & $(-0.0015,-0.037,0.0179)$ \\
\hline 40 & 0 & 2 & $(-0.1078,-0.0933,0.0299)$ & $(-0.1078,-0.0933,0.0299)$ \\
\hline 45 & 0 & 2 & $(-0.0849,11.8834,-3.0959)$ & $(0.003,0.0794,-0.116)$ \\
\hline 50 & 0 & 2 & $(1.4485,6.6851,-3.1534)$ & $(-0.0494,-0.0104,-0.0127)$ \\
\hline 54 & 0 & 2 & $(2.9768,0.2554,-3.1461)$ & $(0.0146,0.054,-0.003)$ \\
\hline \hline
\end{tabular}


Table B-41: Error for Localisation Symmetric Map (UCPF-MCL) Case 5

\begin{tabular}{|c|c|c|c|c|}
\hline \hline$\#$ & $\mathrm{R}$ & $\mathrm{C}$ & Most Likely Sample & Closest Cluster \\
\hline \hline 1 & 0 & 8 & $(4.0738,-6.5104,-1.4934)$ & $(-0.0661,-0.1128,-0.0423)$ \\
\hline 2 & 0 & 7 & $(2.9653,-6.9854,-1.5382)$ & $(-0.4025,-0.074,-0.0901)$ \\
\hline 3 & 0 & 7 & $(0.1233,0.363,-1.087)$ & $(0.1233,0.363,-1.087)$ \\
\hline 4 & 0 & 7 & $(0.1652,0.4649,-0.0991)$ & $(0.1652,0.4649,-0.0991)$ \\
\hline 5 & 0 & 7 & $(2.0058,-1.3117,-3.0976)$ & $(-0.0967,0.2383,-0.0066)$ \\
\hline 10 & 0 & 2 & $(-0.2325,0.0229,0.0136)$ & $(-0.2325,0.0229,0.0136)$ \\
\hline 15 & 0 & 2 & $(-2.3007,-1.0429,3.1048)$ & $(-0.1476,-0.1123,-0.035)$ \\
\hline 20 & 0 & 2 & $(2.9771,-0.1664,-3.1614)$ & $(0.0384,0.0522,-0.0082)$ \\
\hline 25 & 0 & 2 & $(2.0606,-9.0096,-3.2113)$ & $(-0.0293,-0.115,-0.0585)$ \\
\hline 30 & 0 & 2 & $(1.6761,-11.9796,3.265)$ & $(0.1538,-0.0539,0.1057)$ \\
\hline 35 & 0 & 2 & $(0.0603,-0.1208,-0.0265)$ & $(0.0603,-0.1208,-0.0265)$ \\
\hline 40 & 0 & 2 & $(0.0419,-0.1365,0.0038)$ & $(0.0419,-0.1365,0.0038)$ \\
\hline 45 & 0 & 2 & $(0.0941,0.0771,-0.0545)$ & $(0.0941,0.0771,-0.0545)$ \\
\hline 50 & 0 & 2 & $(-0.068,0.1069,-0.039)$ & $(-0.068,0.1069,-0.039)$ \\
\hline 54 & 0 & 2 & $(0.0117,0.0926,0.0006)$ & $(0.0117,0.0926,0.0006)$ \\
\hline \hline
\end{tabular}

Table B-42: Error for Localisation Symmetric Map (UCPF-MCL) Case 6

\begin{tabular}{|c|c|c|c|c|}
\hline \hline$\#$ & $\mathrm{R}$ & $\mathrm{C}$ & Most Likely Sample & Closest Cluster \\
\hline \hline 1 & 0 & 9 & $(4.1304,-6.6493,-1.5575)$ & $(-0.5475,-0.1157,-0.0553)$ \\
\hline 2 & 0 & 6 & $(-0.2854,-0.1655,-0.0597)$ & $(-0.2854,-0.1655,-0.0597)$ \\
\hline 3 & 0 & 6 & $(5.5009,-1.1156,-3.187)$ & $(0.0396,-0.1005,-0.0475)$ \\
\hline 4 & 0 & 5 & $(3.4598,-1.2018,-3.1791)$ & $(0.2206,-0.0503,-0.0332)$ \\
\hline 5 & 0 & 4 & $(0.1407,0.0036,-0.0327)$ & $(0.1407,0.0036,-0.0327)$ \\
\hline 10 & 0 & 2 & $(-0.1554,0.249,-0.014)$ & $(-0.1554,0.249,-0.014)$ \\
\hline 15 & 0 & 2 & $(-0.1056,0.1339,-0.0314)$ & $(-0.1056,0.1339,-0.0314)$ \\
\hline 20 & 0 & 2 & $(0.1977,0.31,-0.0338)$ & $(0.1977,0.31,-0.0338)$ \\
\hline 25 & 0 & 2 & $(2.0175,-9.0723,-3.1729)$ & $(0.0575,-0.065,-0.0198)$ \\
\hline 30 & 0 & 2 & $(1.6096,-11.9263,3.2754)$ & $(0.2563,-0.1026,0.128)$ \\
\hline 35 & 0 & 2 & $(1.5382,-2.8589,3.1662)$ & $(0.0279,-0.0175,0.0259)$ \\
\hline 40 & 0 & 2 & $(-0.1074,-0.0865,0.0361)$ & $(-0.1074,-0.0865,0.0361)$ \\
\hline 45 & 0 & 2 & $(-0.0227,0.0838,-0.0697)$ & $(-0.0227,0.0838,-0.0697)$ \\
\hline 50 & 0 & 2 & $(1.4668,6.6678,-3.149)$ & $(-0.0643,0.0299,-0.0058)$ \\
\hline 54 & 0 & 2 & $(0.0208,0.0391,-0.0053)$ & $(0.0208,0.0391,-0.0053)$ \\
\hline \hline
\end{tabular}


Table B-43: Error for Localisation Symmetric Map (UCPF-MCL) Case 7

\begin{tabular}{|c|c|c|c|c|}
\hline \hline$\#$ & $\mathrm{R}$ & $\mathrm{C}$ & Most Likely Sample & Closest Cluster \\
\hline \hline 1 & 0 & 9 & $(4.1307,-6.652,-1.5521)$ & $(-0.5472,-0.1184,-0.0499)$ \\
\hline 2 & 0 & 6 & $(-0.2784,-0.1729,-0.0466)$ & $(-0.2784,-0.1729,-0.0466)$ \\
\hline 3 & 0 & 6 & $(0.0315,-0.1302,-0.0208)$ & $(0.0315,-0.1302,-0.0208)$ \\
\hline 4 & 0 & 5 & $(0.0028,-0.1135,-0.0003)$ & $(0.0028,-0.1135,-0.0003)$ \\
\hline 5 & 0 & 5 & $(0.0157,-0.1071,0.0007)$ & $(0.0157,-0.1071,0.0007)$ \\
\hline 10 & 0 & 2 & $(-6.1115,-1.9035,3.1315)$ & $(0.0248,-0.1379,0.0008)$ \\
\hline 15 & 0 & 2 & $(-2.4147,-1.1955,3.1263)$ & $(-0.0589,-0.1072,-0.0119)$ \\
\hline 20 & 0 & 2 & $(3.1781,-0.1006,-3.1523)$ & $(-0.2008,-0.1095,-0.0271)$ \\
\hline 25 & 0 & 2 & $(0.0439,0.0295,0.0389)$ & $(0.0439,0.0295,0.0389)$ \\
\hline 30 & 0 & 2 & $(1.5581,-11.9543,3.2439)$ & $(0.3108,-0.1001,0.1151)$ \\
\hline 35 & 0 & 2 & $(1.519,-2.8995,3.1631)$ & $(0.0083,-0.0116,0.0249)$ \\
\hline 40 & 0 & 2 & $(0.9232,6.36,3.1835)$ & $(-0.1457,-0.0847,0.0444)$ \\
\hline 45 & 0 & 2 & $(-0.2574,0.0477,0.0206)$ & $(-0.2574,0.0477,0.0206)$ \\
\hline 50 & 0 & 2 & $(1.3459,6.671,-3.1211)$ & $(-0.1588,0.0452,0.0226)$ \\
\hline 54 & 0 & 2 & $(-0.1691,0.0682,0.0115)$ & $(-0.1691,0.0682,0.0115)$ \\
\hline \hline
\end{tabular}

Table B-44: Error for Localisation Symmetric Map (UCPF-MCL) Case 8

\begin{tabular}{|c|c|c|c|c|}
\hline \hline$\#$ & $\mathrm{R}$ & $\mathrm{C}$ & Most Likely Sample & Closest Cluster \\
\hline \hline 1 & 0 & 9 & $(4.1361,-6.6468,-1.5515)$ & $(-0.5797,0.0752,-0.0376)$ \\
\hline 2 & 0 & 7 & $(7.6492,-1.016,-3.1595)$ & $(-0.1634,0.2145,-0.0453)$ \\
\hline 3 & 0 & 7 & $(5.5126,-1.0972,-3.1358)$ & $(0.0756,0.1503,-0.3535)$ \\
\hline 4 & 0 & 7 & $(-0.0037,0.1308,-0.0012)$ & $(-0.0037,0.1308,-0.0012)$ \\
\hline 5 & 0 & 7 & $(1.8341,-1.2956,-3.1187)$ & $(0.0221,0.1289,0.0011)$ \\
\hline 10 & 0 & 2 & $(-0.0025,-0.0077,-0.0149)$ & $(-0.0025,-0.0077,-0.0149)$ \\
\hline 15 & 0 & 2 & $(-0.0657,0.0032,-0.0125)$ & $(-0.0657,0.0032,-0.0125)$ \\
\hline 20 & 0 & 2 & $(3.1574,-0.0337,-3.1693)$ & $(-0.1452,0.0054,-0.0114)$ \\
\hline 25 & 0 & 2 & $(2.4004,-9.2384,-3.1078)$ & $(0.0278,0.0293,0.0286)$ \\
\hline 30 & 0 & 2 & $(0.2985,-0.1023,0.11)$ & $(0.2985,-0.1023,0.11)$ \\
\hline 35 & 0 & 2 & $(1.5112,-2.9365,3.1611)$ & $(0.0188,0.0274,0.0225)$ \\
\hline 40 & 0 & 2 & $(-0.1185,-0.1222,0.0415)$ & $(-0.1185,-0.1222,0.0415)$ \\
\hline 45 & 0 & 2 & $(0.3171,12.0587,3.1524)$ & $(-0.2219,0.0597,0.0102)$ \\
\hline 50 & 0 & 2 & $(1.3124,6.6822,-3.1235)$ & $(-0.112,0.0334,0.0183)$ \\
\hline 54 & 0 & 2 & $(3.0199,0.1639,-3.132)$ & $(-0.1364,0.0836,0.0096)$ \\
\hline \hline
\end{tabular}


Table B-45: Error for Localisation Symmetric Map (UCPF-MCL) Case 9

\begin{tabular}{|c|c|c|c|c|}
\hline \hline$\#$ & $\mathrm{R}$ & $\mathrm{C}$ & Most Likely Sample & Closest Cluster \\
\hline \hline 1 & 0 & 8 & $(9.8237,-1.0651,-3.0889)$ & $(-0.0657,-0.1155,-0.0369)$ \\
\hline 2 & 0 & 7 & $(8.8523,-1.4648,-2.9301)$ & $(-0.6253,0.0214,-0.077)$ \\
\hline 3 & 0 & 7 & $(0.1125,0.3757,-0.0321)$ & $(0.1125,0.3757,-0.0321)$ \\
\hline 4 & 0 & 7 & $(0.256,0.3036,-0.0445)$ & $(0.256,0.3036,-0.0445)$ \\
\hline 5 & 0 & 7 & $(-0.1128,0.1812,-1.9881)$ & $(-0.1128,0.1812,-1.9881)$ \\
\hline 10 & 0 & 1 & $(-0.0144,-0.0292,-0.0255)$ & $(-0.0144,-0.0292,-0.0255)$ \\
\hline 15 & 0 & 1 & $(-0.074,0.0674,-0.0124)$ & $(-0.074,0.0674,-0.0124)$ \\
\hline 20 & 0 & 1 & $(-0.0198,0.1029,-0.0007)$ & $(-0.0198,0.1029,-0.0007)$ \\
\hline 25 & 0 & 1 & $(0.235,0.3035,0.0584)$ & $(0.235,0.3035,0.0584)$ \\
\hline 30 & 0 & 1 & $(0.1095,0.1429,0.0799)$ & $(0.1095,0.1429,0.0799)$ \\
\hline 35 & 1 & 1 & $(2.6627,-0.8008,-1.3872)$ & $(2.6627,-0.8008,-1.3872)$ \\
\hline 40 & 1 & 1 & $(-0.0016,-0.1406,0.0212)$ & $(-0.0016,-0.1406,0.0212)$ \\
\hline 45 & 1 & 1 & $(-0.0822,0.0724,-0.0305)$ & $(-0.0822,0.0724,-0.0305)$ \\
\hline 50 & 1 & 1 & $(-0.1136,0.0173,0.0205)$ & $(-0.1136,0.0173,0.0205)$ \\
\hline 54 & 1 & 1 & $(-0.09,0.0541,-0.0045)$ & $(-0.09,0.0541,-0.0045)$ \\
\hline \hline
\end{tabular}




\section{B.2 Office Map Results}

\section{B.2.1 MCL}

Table B-46: Error for Localisation Office Map (MCL) Case 1

\begin{tabular}{|c|c|c|c|c|c|}
\hline$\#$ & $\mathrm{R}$ & $\mathrm{C}$ & Most Likely Cluster & Most Likely Sample & t Cluster \\
\hline 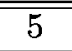 & 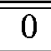 & 1 & 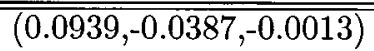 & $\overline{(0.0527,-0.0734,0.0041)}$ & $(0.0527,-0.0734,0.0041)$ \\
\hline 10 & 0 & 1 & $(0.0038,-0.1238,-0.0066)$ & $(-0.0685,-0.0446,-0.0066)$ & $(-0.0685,-0.0446,-0.0066)$ \\
\hline 15 & 0 & 1 & $(0.0199,-0.0116,-0.0073)$ & $(0.0223,-0.0195,-0.0067)$ & $(0.0199,-0.0116,-0.0073)$ \\
\hline 20 & 0 & 1 & $(0.7303,0.0532,0.06)$ & $(0.3648,0.0411,0.0592)$ & $(0.3648,0.0411,0.0592)$ \\
\hline 25 & 0 & 1 & $(1.383,0.1166,0.0308)$ & $(1.3894,0.1189,0.0307)$ & $(1.383,0.1166,0.0308)$ \\
\hline 30 & 1 & 1 & $(0.0067,0.1494,0.0349)$ & $(0.0347,0.1427,0.0172)$ & $(0.0347,0.1427,0.0172)$ \\
\hline 35 & 1 & 1 & $(-0.016,0.0539,-0.0091)$ & $(-0.0198,0.0959,-0.0085)$ & $(-0.016,0.0539,-0.0091)$ \\
\hline 40 & 1 & 1 & $(0.1713,-0.0177,0.0295)$ & $(0.1694,-0.0567,-0.0285)$ & $(0.1713,-0.0177,0.0295)$ \\
\hline 45 & 1 & 1 & $(0.0546,-0.0821,0.0178)$ & $(0.1,-0.0673,0.0128)$ & $(0.0546,-0.0821,0.0178)$ \\
\hline 50 & 1 & 1 & $(0.104,0.0187,-1.7858)$ & $(0.0706,-0.0093,-0.0081)$ & $(0.0706,-0.0093,-0.0081)$ \\
\hline 55 & 1 & 1 & $(0.5934,-0.0007,-3.3224)$ & $(0.9713,0.0118,0)$ & $(0.9713,0.0118,0)$ \\
\hline 60 & 1 & 1 & $(0.0602,-0.011,-0.0015)$ & $(-0.0183,0.0205,-0.005)$ & $(-0.0183,0.0205,-0.005)$ \\
\hline 65 & 1 & 1 & $(-0.188,0.4557,0.0733)$ & $(-0.2505,0.4204,0.0878)$ & $204,0.0878)$ \\
\hline 70 & 1 & 1 & $(-0.239,0.3242,-0.0$ & $3896,-0.0174)$ & $(-0.239,0$ \\
\hline 75 & 2 & 7 & $-0.7447)$ & $(0.1962,-7.79,-1.5541)$ & $(0.0825,0$ \\
\hline 80 & 2 & 1 & $.0347)$ & $(0.2451$ & $(0.2365$ \\
\hline 85 & 2 & 1 & $7,0.0338)$ & $(0.3416,-1.0278,0.0338)$ & $(0.3464,-0.9157,0.0338)$ \\
\hline 90 & 2 & 1 & $(0.4$ & $6,0.0328)$ & $(0.4499,-2.0964,0.0328)$ \\
\hline 95 & 3 & 1 & $(-0$. & $(-0.0387,-1$ & $(-0.0353$, \\
\hline 100 & 3 & 1 & $(-0.0709,0.1154,-0.0157)$ & $(-0.0693,0.1347,-0.0155)$ & $(-0.0709,0.1154,-0.0157)$ \\
\hline 105 & 3 & 1 & $(-0.2362,0.0098,0.0493)$ & $(-0.2719,0.0149,0.0516)$ & $(-0.2362,0.0098,0.0493)$ \\
\hline 110 & 3 & 4 & $745,0.0203)$ & $(-0.1918,0.1127,0.0257)$ & $(0.0018,0.0745,0.0203)$ \\
\hline 115 & 3 & 1 & $(-0.2251,0.1141,0.0138)$ & $(-0.3305,0.1113,0.0137)$ & $(-0.2251,0.1141,0.0138)$ \\
\hline 120 & 3 & 1 & $(-0.7206,0.1637,0.0123)$ & $(-1.0916,0.1557,0.0123)$ & $(-0.7206,0.1637,0.0123)$ \\
\hline 125 & 3 & 1 & $(-0.3617,0.2256,0.0116)$ & $(-0.4062,0.2246,0.0116)$ & $(-0.3617,0.2256,0.0116)$ \\
\hline 130 & 3 & 1 & $(-0.5957,0.2701,0.0106)$ & $(-0.6179,0.2696,0.0106)$ & $(-0.5957,0.2701,0.0106)$ \\
\hline 135 & 4 & 3 & $(0.1188,-0.3203,0.0817)$ & $(0.1188,-0.3203,0.0817)$ & $(0.1188,-0.3203,0.0817)$ \\
\hline 140 & 4 & 1 & $(0.1626,-0.6133,0.0581)$ & $(0.1584,-0.5728,0.0632)$ & $(0.1584,-0.5728,0.0632)$ \\
\hline 145 & 4 & 1 & $(-0.0187,-0.1327,0.0612)$ & $(-0.0072,-0.0282,0.061)$ & $(-0.0072,-0.0282,0.061)$ \\
\hline 150 & 4 & 1 & $(0.1825,0.0445,0.0229)$ & $(0.3885,-0.0163,0.0463)$ & $(0.1825,0.0445,0.0229)$ \\
\hline 155 & 4 & 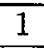 & $(-0.0049,0.0984,0.0023)$ & $(0.0796,0.1367,0.0115)$ & $(-0.0049,0.0984,0.0023)$ \\
\hline 160 & 4 & 2 & $(0.0001,0.0484,0.0012)$ & $(0.018,0.0563,-0.0006)$ & $(0.0001,0.0484,0.0012)$ \\
\hline 165 & 4 & 1 & $(0.1989,-0.038,0.028)$ & $(0.1996,-0.0176,0.0393)$ & $(0.1996,-0.0176,0.0393)$ \\
\hline 170 & 4 & 1 & $(0.0643,-0.1727,0.0392)$ & $(0.0561,-0.2471,0.0436)$ & $(0.0643,-0.1727,0.0392)$ \\
\hline
\end{tabular}


Table B-47: Error for Localisation Office Map (MCL) Case 2

\begin{tabular}{|c|c|c|c|c|c|}
\hline$\#$ & $\mathrm{R}$ & $\mathrm{C}$ & Most Likely Cluster & Most Likely Sample & Closest Cluster \\
\hline$\overline{5}$ & 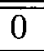 & $\overline{7}$ & $(0.016,6.035,-0.0043)$ & $\overline{(0.063,6.0812,-0.0016)}$ & $\overline{(-0.1996,-0.0239,-0.07)}$ \\
\hline 10 & 0 & 1 & $(0.372,23.6908,0.0558)$ & $(0.2666,23.7735,0.0589)$ & $(0.372,23.6908,0.0558)$ \\
\hline 15 & 0 & 1 & $(0.2066,23.4024,0.0297)$ & $(0.2134,23.4382,0.0343)$ & $(0.2066,23.4024,0.0297)$ \\
\hline 20 & 0 & 1 & $(1.1826,24.6658,-0.0914)$ & $(0.9028,24.5689,-0.0914)$ & $(0.9028,24.5689,-0.0914)$ \\
\hline 25 & 0 & 1 & $(1.6246,25.3487,-0.1046)$ & $(1.5857,25.3352,-0.1046)$ & $(1.5857,25.3352,-0.1046)$ \\
\hline 30 & 0 & 1 & $(1.3437,25.9291,-0.1815)$ & $(1.3737,25.9272,-0.1303)$ & $(1.3737,25.9272,-0.1303)$ \\
\hline 35 & 0 & 1 & $(0.2661,24.9167,-0.4121)$ & $(0.2852,24.8804,-0.4115)$ & $(0.2852,24.8804,-0.4115)$ \\
\hline 40 & 0 & 1 & $(-0.1606,25.5423,-0.3801)$ & $(-0.1593,25.5485,-0.3827)$ & $(-0.1606,25.5423,-0.3801)$ \\
\hline 45 & 1 & 1 & $(0.2717,0.0371,-0.0015)$ & $(0.2388,0.2851,-0.0445)$ & $(0.2717,0.0371,-0.0015)$ \\
\hline 50 & 1 & 1 & $(0.6419,0.0482,1.7813)$ & $(0.7797,0.068,6.2224)$ & $(0.6419,0.0482,1.7813)$ \\
\hline 55 & 1 & 1 & $(1.1223,0.0329,0.2392)$ & $(1.484,0.006,-0.013)$ & $(1.1223,0.0329,0.2392)$ \\
\hline 60 & 1 & 1 & $(0.7448,0.0254,-0.0156)$ & $(0.2738,-0.0862,0.0055)$ & $(0.2738,-0.0862,0.0055)$ \\
\hline 65 & 1 & 1 & $(-0.0864,0.4547,0.0294)$ & $(-0.0996,0.4147,0.0153)$ & $(-0.0996,0.4147,0.0153)$ \\
\hline 70 & 1 & 1 & $(-0.1632,0.9371,-0.0547)$ & $(-0.1154,0.8391,-0.0552)$ & $(-0.1154,0.8391,-0.0552)$ \\
\hline 75 & 1 & 1 & $(-0.0011,0.2502,0.076)$ & $(-0.007,0.2186,0.0762)$ & $(-0.007,0.2186,0.0762)$ \\
\hline 80 & 1 & 1 & $(0.3205,0.2373,0.0803)$ & $(0.3171,0.2021,0.0803)$ & $(0.3171,0.2021,0.0803)$ \\
\hline 85 & 1 & 1 & $(0.6416,0.3098,0.0587)$ & $(0.6954,0.8527,0.0587)$ & $(0.6416,0.3098,0.0587)$ \\
\hline 90 & 1 & 1 & $(0.7939,-1.0529,0.0448)$ & $(0.7705,-1.2887,0.0448)$ & $(0.7939,-1.0529,0.0448)$ \\
\hline 95 & 1 & 1 & $(0.953,-2.463,0.0809)$ & $(0.9416,-2.578,0.0809)$ & $(0.953,-2.463,0.0809)$ \\
\hline 100 & 1 & 1 & $(1.2976,-1.4067,0.0542)$ & $(1.3065,-1.2978,0.0543)$ & $(1.3065,-1.2978,0.0543)$ \\
\hline 105 & 1 & 1 & $(0.7495,-1.0616,-0.0766)$ & $(0.7829,-0.9665,-0.0875)$ & $(0.7829,-0.9665,-0.0875)$ \\
\hline 110 & 1 & 1 & $(-0.7873,-1.0711,-0.0601)$ & $(-0.7634,-1.0703,-0.0601)$ & $(-0.7634,-1.0703,-0.0601)$ \\
\hline 115 & 1 & 1 & $(-0.1072,-1.2655,-0.0693)$ & $(-0.1674,-1.2701,-0.0693)$ & $(-0.1072,-1.2655,-0.0693)$ \\
\hline 120 & 1 & 1 & $(0.2599,-1.5467,-0.0865)$ & $(0.4085,-1.5332,-0.0864)$ & $(0.2599,-1.5467,-0.0865)$ \\
\hline 125 & 1 & 1 & $(1.6618,-1.8873,-0.1074)$ & $(1.7277,-1.877,-0.1074)$ & $(1.6618,-1.8873,-0.1074)$ \\
\hline 130 & 2 & 1 & $(11.338,-15.212,-3.053)$ & $(21.75,-37.121,0.1305)$ & $(11.339,-15.213,-3.053)$ \\
\hline 135 & 2 & 2 & $(5.6876,0.7109,3.1221)$ & $(5.847,0.8193,3.1272)$ & $(5.6876,0.7109,3.1221)$ \\
\hline 140 & 2 & 1 & $(9.1677,6.562,2.9767)$ & $(9.1651,6.57,2.9746)$ & $(9.1677,6.562,2.9767)$ \\
\hline 145 & 2 & 1 & $(9.257,14.0696,3.0002)$ & $(9.2591,14.0507,3.0002)$ & $(9.2591,14.0507,3.0002)$ \\
\hline 150 & 2 & 1 & $(4.8238,17.6954,2.8572)$ & $(4.8483,17.6978,2.8581)$ & $(4.8238,17.6954,2.8572)$ \\
\hline 155 & 3 & 8 & $(0.8427,-1.1291,-1.1919)$ & $(-0.6447,0.8079,0.1769)$ & $(-0.6447,0.8079,0.1769)$ \\
\hline 160 & 3 & 1 & $(0.076,0.1817,-0.0073)$ & $(0.081,-0.0084,0.0051)$ & $(0.081,-0.0084,0.0051)$ \\
\hline 165 & 3 & 1 & $(0.2668,0.0455,-0.0007)$ & $(0.2668,0.1187,0.0233)$ & $(0.2668,0.0455,-0.0007)$ \\
\hline 170 & 3 & 1 & $(0.1571,-0.298,0.0618)$ & $(0.2231,-0.1373,0.0141)$ & $(0.2231,-0.1373,0.0141)$ \\
\hline 175 & 3 & 1 & $(-0.0213,-1.0465,0.1303)$ & $(0.0372,-1.0027,0.1337)$ & $(0.0372,-1.0027,0.1337)$ \\
\hline 180 & 3 & 1 & $(0.0667,-0.4686,0.0915)$ & $(-0.0023,-0.5654,0.0939)$ & $(0.0667,-0.4686,0.0915)$ \\
\hline 185 & 3 & 1 & $(-0.3971,-0.6835,0.1172)$ & $(-0.4811,-0.4845,0.1181)$ & $(-0.4811,-0.4845,0.1181)$ \\
\hline 190 & 3 & 1 & $(-1.2268,-0.3697,0.1348)$ & $(-1.2599,-0.3276,0.1212)$ & $(-1.2268,-0.3697,0.1348)$ \\
\hline
\end{tabular}


Table B-48: Error for Localisation Office Map (MCL) Case 3

\begin{tabular}{|c|c|c|c|c|c|}
\hline$\#$ & $\mathrm{R}$ & $\mathrm{C}$ & Most Likely Cluster & Most Likely Sample & Closest C \\
\hline$\overline{5}$ & $\overline{00}$ & $\overline{1} 1$ & 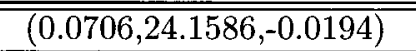 & 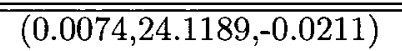 & 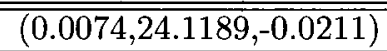 \\
\hline 10 & 1 & 1 & $(0.349,0.1448,0.0472)$ & $(0.3632,0.1274,0.0472)$ & $(0.349,0.1448,0.0472)$ \\
\hline 15 & 1 & 1 & $(0.2418,0.035,0.0465)$ & $(0.2688,0.0054,0.0465)$ & $(0.2418,0.035,0.0465)$ \\
\hline 20 & 1 & 1 & $(0.9508,0.0551,0.0734)$ & $(0.9405,0.0469,0.0729)$ & $(0.9405,0.0469,0.0729)$ \\
\hline 25 & $\overline{1}$ & 1 & $(2.5175,0.2022,0.0508)$ & $(2.5175,0.2022,0.0508)$ & $(2.5175,0.2022,0.0508)$ \\
\hline 30 & 3 & 1 & $(3.5065,16.687,-5.49)$ & $(-9.5629,26.224,-6.3887)$ & $(3.5065,16.687,-5.49)$ \\
\hline 35 & 4 & 2 & $(22.2874,-0.1619,-4.6757)$ & $(22.2526,-0.1604,-4.6757)$ & $(22.2526,-0.1604,-4.6757)$ \\
\hline 40 & 4 & 1 & $(23.4405,-1.8968,1.2932)$ & $(23.4395,-1.897,1.2673)$ & $(23.4395,-1.897,1.2673)$ \\
\hline 45 & 5 & 1 & $(3.8149,16.2589,-1.6833)$ & $(28.6215,31.5634,2.644)$ & $(3.8149,16.2589,-1.6833)$ \\
\hline 50 & 5 & 1 & $(0.0679,-0.0134,-0.0457)$ & $(0.2694,-0.0302,-0.0118)$ & $(0.0679,-0.0134,-0.0457)$ \\
\hline 55 & 5 & 1 & $(0.5562,0.0091,-0.0103)$ & $(0.913,0.0203,-0.0108)$ & $(0.5562,0.0091,-0.0103)$ \\
\hline 60 & 5 & 1 & $(0.0682,-0.0015,-0.0082)$ & $(0.0923,-0.0288,-0.0059)$ & $(0.0682,-0.0015,-0.0082)$ \\
\hline 65 & 5 & 1 & $(-0.2021,0.4564,0.0774)$ & $(-0.3351,0.4515,0.1098)$ & $(-0.2021,0.4564,0.0774)$ \\
\hline 70 & 5 & 1 & $(-0.2386,0.3083,-0.0155)$ & $(-0.2369,0.0417,-0.0154)$ & $(-0.2369,0.0417,-0.0154)$ \\
\hline 75 & 6 & 3 & $(-29.0353,-8.8721,-0.0302)$ & $(0.0989,18.2949,-0.1128)$ & $(-0.2603,-7.821,-1.4375)$ \\
\hline 80 & 7 & 4 & $(-0.0572,6.6528,-0.537)$ & $(-0.0764,5.7265,0.031)$ & $(-0.0764,5.7265,0.031)$ \\
\hline 85 & 7 & 2 & $(0.0432,5.9212,0.0225)$ & $(0.065,5.8221,0.0302)$ & $(-0.4657,-2.7525,-3.1137)$ \\
\hline 90 & 8 & 1 & $(-11.795,-4.273,-4.748)$ & $(-21.780,4.017,-14.583)$ & $(-11.795,-4.273,-4.748)$ \\
\hline 95 & 8 & 1 & $(-0.0168,0.1214,0.0098)$ & $(-0.016,0.0966,0.0101)$ & $(-0.016,0.0966,0.0101)$ \\
\hline 100 & 8 & 1 & $(0.0477,0.1084,0.008)$ & $(0.0449,0.11,0.0075)$ & $(0.0477,0.1084,0.008)$ \\
\hline 105 & 8 & 1 & $(-0.1941,-0.0686,0.0493)$ & $(-0.2081,-0.0614,0.059)$ & $(-0.1941,-0.0686,0.0493)$ \\
\hline 110 & 8 & 1 & $(0.0192,0.0288,0.0264)$ & $(-0.0257,0.0401,0.0296)$ & $(0.0192,0.0288,0.0264)$ \\
\hline 115 & 8 & 1 & $(-0.2028,0.0363,0.0156)$ & $(-0.2205,0.0348,0.0137)$ & $(-0.2028,0.0363,0.0156)$ \\
\hline 120 & 8 & 1 & $(-0.5487,0.0415,0.0111)$ & $(-0.4053,0.0447,0.0111)$ & $(-0.4053,0.0447,0.0111)$ \\
\hline 125 & 8 & 1 & $(-0.1825,0.0983,0.0104)$ & $(-0.2109,0.0976,0.0104)$ & $(-0.1825,0.0983,0.0104)$ \\
\hline 130 & 8 & 1 & $(-0.5071,0.1349,0.0094)$ & $(-0.5898,0.133,0.0094)$ & $(-0.5071,0.1349,0.0094)$ \\
\hline 135 & 9 & 1 & $(15.9461,-14.5805,-2.6968)$ & $(4.3998,8.2938,-4.5336)$ & $(4.3998,8.2938,-4.5336)$ \\
\hline 140 & 9 & 1 & $(0.0426,-0.4072,0.0153)$ & $(0.0516,-0.3004,0.0135)$ & $(0.0516,-0.3004,0.0135)$ \\
\hline 145 & 9 & 1 & $(-0.0123,-0.3579,0.0201)$ & $(0.0076,-0.4586,0.0145)$ & $(-0.0123,-0.3579,0.0201)$ \\
\hline 150 & 9 & 1 & $(0.1562,0.0791,0.0239)$ & $(0.0075,0.1053,0.018)$ & $(0.0075,0.1053,0.018)$ \\
\hline 155 & 9 & 1 & $(-0.0076,0.1039,0.003)$ & $(0.0384,0.0132,-0.0047)$ & $(0.0384,0.0132,-0.0047)$ \\
\hline 160 & 9 & 1 & $(-0.0022,0.0488,0.0016)$ & $(-0.0369,0.0367,0.0014)$ & $(-0.0022,0.0488,0.0016)$ \\
\hline 165 & 9 & 1 & $(0.1985,-0.0376,0.0279)$ & $(0.1936,-0.0342,0.031)$ & $(0.1936,-0.0342,0.031)$ \\
\hline 170 & 9 & 1 & $(0.0641,-0.1724,0.0394)$ & $(0.0405,0.0194,0.0171)$ & $(0.0405,0.0194,0.0171)$ \\
\hline 175 & 9 & 1 & $(0.0321,-0.5018,0.034)$ & $(0.0149,-0.2445,0.0302)$ & $(0.0149,-0.2445,0.0302)$ \\
\hline 180 & 9 & 1 & $(-0.0002,-0.0787,0.0147)$ & $(-0.01,-0.1007,0.0157)$ & $(-0.0002,-0.0787,0.0147)$ \\
\hline 185 & 9 & 1 & $(0.043,-0.0269,0.0077)$ & $(0.0624,0.0232,0.0049)$ & $(0.043,-0.0269,0.0077)$ \\
\hline 190 & 9 & 1 & $(-0.4423,-0.0014,-0.0028)$ & $(-0.43,0.0223,0.026)$ & $(-0.43,0.0223,0.026)$ \\
\hline
\end{tabular}


Table B-49: Error for Localisation Office Map (MCL) Case 4

\begin{tabular}{|c|c|c|c|c|c|}
\hline$\#$ & $\mathrm{R}$ & $\mathrm{C}$ & Most Likely Cluster & Most Likely Sample & Closest C \\
\hline$\overline{5}$ & $\overline{0} 0$ & $\overline{4}$ & $(-0.0725,24.1501,0.0553)$ & $(-0.262,24.0089,0.055)$ & $(-0.2753,-0.0007,-0.058)$ \\
\hline 10 & 0 & $\overline{1}$ & $(0.3789,23.7675,0.0572)$ & $(0.2471,23.6309,0.0606)$ & $(0.2471,23.6309,0.0606)$ \\
\hline 15 & 0 & $\overline{1}$ & $(0.1775,23.3886,0.0375)$ & $(0.1912,23.3749,0.0375)$ & $(0.1912,23.3749,0.0375)$ \\
\hline 20 & 0 & 1 & $(1.147,24.4515,-0.0705)$ & $(1.4313,24.531,-0.0676)$ & $(1.147,24.4515,-0.0705)$ \\
\hline 25 & 0 & $\overline{1}$ & $(1.3287,24.9579,-0.0863)$ & $(1.2176,24.9227,-0.0864)$ & $(1.2176,24.9227,-0.0864)$ \\
\hline 30 & 0 & 1 & $(0.7805,25.3415,-0.183)$ & $(0.749,25.3036,-0.0797)$ & $(0.749,25.3036,-0.0797)$ \\
\hline 35 & 0 & $\overline{1}$ & $(-0.0372,25.0147,-0.3248)$ & $(-0.0331,25.0022,-0.325)$ & $(-0.0331,25.0022,-0.325)$ \\
\hline 40 & 0 & 1 & $(-0.3102,25.5938,-0.3578)$ & $(-0.3107,25.5956,-0.3699)$ & $(-0.3102,25.5938,-0.3578)$ \\
\hline 45 & 1 & 1 & $(5.18,31.8805,0.0874)$ & $(5.1731,31.8621,0.0877)$ & $(5.1731,31.8621,0.0877)$ \\
\hline 50 & 1 & 1 & $(5.5463,32.289,2.8319)$ & $(5.5664,32.2871,6.2498)$ & $(5.5463,32.289,2.8319)$ \\
\hline 55 & 1 & 1 & $(5.1653,32.4757,1.9143)$ & $(5.1489,32.4846,-0.0532)$ & $(5.1489,32.4846,-0.0532)$ \\
\hline 60 & 1 & $\overline{1}$ & $(6.6871,32.6565,0.0043)$ & $(6.6976,32.6565,-0.0488)$ & $(6.6871,32.6565,0.0043)$ \\
\hline 65 & 1 & 1 & $(7.0604,33.5029,-0.0168)$ & $(7.2931,33.6182,-0.052)$ & $(7.0604,33.5029,-0.0168)$ \\
\hline 70 & 1 & 1 & $(6.6573,32.6228,-0.0552)$ & $(6.6582,32.5277,-0.0552)$ & $(6.6582,32.5277,-0.0552)$ \\
\hline 75 & 1 & $\overline{1}$ & $(6.4429,31.1344,-0.0321)$ & $(6.4429,31.128,-0.0321)$ & $(6.4429,31.128,-0.0321)$ \\
\hline 80 & 1 & $\overline{1}$ & $(6.2716,31.0737,-0.0279)$ & $(6.271,31.1368,-0.0279)$ & $(6.2716,31.0737,-0.0279)$ \\
\hline 85 & 2 & 8 & $(-0.7748,0.6589,-1.666)$ & $(-1.4008,0.6983,-3.0999)$ & $(-0.7748,0.6589,-1.666)$ \\
\hline 90 & 2 & 3 & $5,-0.6466,0.0066)$ & $\overline{0.8399,0.0102)}$ & $(0.1015,-0.6466,0.0066)$ \\
\hline 95 & 2 & $\overline{1}$ & $7,0.034)$ & $(0.2$ & $17,0.034)$ \\
\hline 100 & 2 & $\overline{1}$ & $(0.2172$, & $\overline{46,-0.0041)}$ & $(0.1996,0.2946,-0.0041)$ \\
\hline 105 & 2 & 1 & $(-0.252,0.1405,-0.0404)$ & $(-0.2658,0.0981,-0.0364)$ & $(-0.2658,0.0981,-0.0364)$ \\
\hline 110 & 2 & 1 & $(-0.3863,-0$ & $(-0.5006,-0.1933,-0.0664)$ & $(-0.3863,-0.2152,-0.091)$ \\
\hline 115 & 2 & 1 & $(-0.162,-0.3225,-0.0555)$ & $355,-0.0568)$ & $(-0.162,-0.3225,-0.0555)$ \\
\hline 120 & 2 & 1 & $(-0.4893,-0.5172,-0.0657)$ & $(-0.547,-0.5156,-0.0656)$ & $(-0.4893,-0.5172,-0.0657)$ \\
\hline 125 & $\overline{2}$ & 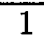 & $(-0.2733,-0.9198,-0.0862)$ & $(-0.1978,-0.8997,-0.0858)$ & $(-0.1978,-0.8997,-0.0858)$ \\
\hline 130 & 2 & 1 & $(0.9786,-1.2278,-0.1125)$ & $(0.8278,-1.2245,-0.1124)$ & $(0.8278,-1.2245,-0.1124)$ \\
\hline 135 & 2 & $\overline{1}$ & $(2.1671,-1.8227,-0.1491)$ & $(2.271,-1.7337,-0.1368)$ & $(2.1671,-1.8227,-0.1491)$ \\
\hline 140 & 3 & $\overline{3}$ & $(-0.1043,-11.6598,-0.0779)$ & $(-0.1061,-11.6569,-0.0768)$ & $(-0.0454,0.1666,-0.0739)$ \\
\hline 145 & 3 & $\overline{1}$ & $(0.2531,-0.4641,-0.0455)$ & $(0.2678,-0.4958,-0.0474)$ & $(0.2531,-0.4641,-0.0455)$ \\
\hline 150 & 3 & 1 & $(0.5261,0.0118,0.0536)$ & $(0.5803,0.0405,0.0575)$ & $(0.5261,0.0118,0.0536)$ \\
\hline 155 & 3 & 1 & $(0.1982,0.2404,0.0046)$ & $(0.0776,0.3055,0.0503)$ & $(0.1982,0.2404,0.0046)$ \\
\hline 160 & 3 & 1 & $(0.1201,0.4371,-0.0143)$ & $(0.0913,0.1959,0.0001)$ & $(0.0913,0.1959,0.0001)$ \\
\hline 165 & 3 & 1 & $(0.2685,0.0829,0.0025)$ & $(0.3141,-0.0731,0.0042)$ & $(0.2685,0.0829,0.0025)$ \\
\hline 170 & 3 & 1 & $(0.1576,-0.271,0.0647)$ & $(0.2562,-0.131,0.0631)$ & $(0.2562,-0.131,0.0631)$ \\
\hline 175 & 3 & 1 & $(-0.0193,-1.0475,0.1318)$ & $(-0.1447,-1.0058,0.1301)$ & $(-0.1447,-1.0058,0.1301)$ \\
\hline 180 & 3 & $\overline{1}$ & $(-0.0119,-0.4998,0.0952)$ & $(-0.0231,-0.474,0.0935)$ & $(-0.0231,-0.474,0.0935)$ \\
\hline 185 & 3 & 1 & $(-0.5441,-0.7199,0.1213)$ & $(-0.5258,-0.7583,0.1209)$ & $(-0.5441,-0.7199,0.1213)$ \\
\hline 190 & 3 & 1 & $(-1.4011,-0.4216,0.1501)$ & $(-1.2045,-0.4448,0.1127)$ & $(-1.2045,-0.4448,0.1127)$ \\
\hline
\end{tabular}


Table B-50: Error for Localisation Office Map (MCL) Case 5

\begin{tabular}{|c|c|c|c|c|c|}
\hline$\#$ & $\mathrm{R}$ & $\mathrm{C}$ & Most Likely Cluster & Most Likely Sample & Closest Cluster \\
\hline 5 & 1 & 1 & $(0.2821,17.6436,0.0889)$ & $(0.2457,17.6199,0.089)$ & $(0.2457,17.6199,0.089)$ \\
\hline 10 & 3 & 1 & $(19.186,15.791,-1.122)$ & $(26.969,-8.322,7.742)$ & $(19.186,15.791,-1.122)$ \\
\hline 15 & 4 & 1 & $(9.6949,1.7122,3.2954)$ & $(9.6949,1.7122,3.2954)$ & $(9.6949,1.7122,3.2954)$ \\
\hline 20 & 4 & 1 & $(1.7277,1.0935,-2.9803)$ & $(1.6217,1.0858,-2.9802)$ & $(1.6217,1.0858,-2.9802)$ \\
\hline 25 & 4 & 1 & $(-6.1931,-0.2131,-2.9812)$ & $(-6.1931,-0.2131,-2.9812)$ & $(-6.1931,-0.2131,-2.9812)$ \\
\hline 30 & 5 & 1 & $(-15.429,25.554,-1.56)$ & $(-15.409,25.517,-1.556)$ & $(-15.409,25.517,-1.556)$ \\
\hline 35 & 6 & 1 & $(22.121,18.389,-1.437)$ & $(22.128,18.3876,-1.4365)$ & $(22.121,18.389,-1.437)$ \\
\hline 40 & 6 & 1 & $(20.4829,17.7214,4.4043)$ & $(20.4829,17.7215,4.3927)$ & $(20.4829,17.7215,4.3927)$ \\
\hline 45 & 7 & 1 & $(3.7282,16.2131,-2.2812)$ & $(28.622,31.563,25.4776)$ & $(3.7282,16.2131,-2.2812)$ \\
\hline 50 & 7 & 1 & $(-0.0386,0.0114,-3.2407)$ & $(0.0157,-0.0002,-6.2743)$ & $(-0.0386,0.0114,-3.2407)$ \\
\hline 55 & 7 & 1 & $(0.4716,-0.0024,-3.6111)$ & $(0.5441,-0.0187,-6.2793)$ & $(0.4716,-0.0024,-3.6111)$ \\
\hline 60 & 7 & 1 & $(0.0582,-0.009,-0.0008)$ & $(0.0936,-0.0079,-0.0012)$ & $(0.0582,-0.009,-0.0008)$ \\
\hline 65 & 7 & 1 & $(-0.1687,0.4663,0.0734)$ & $(-0.1838,0.4378,0.0794)$ & $(-0.1838,0.4378,0.0794)$ \\
\hline 70 & 8 & 2 & $(-0.1818,35.7808,-3.101)$ & $(-0.1818,35.7808,-3.101)$ & $(0.234,11.9365,-0.055)$ \\
\hline 75 & 9 & 1 & $(-0.6611,25.9703,-3.3711)$ & $(-0.6611,25.9703,-3.3711)$ & $(-0.6611,25.9703,-3.3711)$ \\
\hline 80 & 9 & 1 & $(0.3998,16.4762,-3.37)$ & $(0.3998,16.4762,-3.37)$ & $(0.3998,16.4762,-3.37)$ \\
\hline 85 & 10 & 1 & $(0.1277,-1.2414,-0.0499)$ & $(0.1327,-1.3636,-0.0499)$ & $(0.1277,-1.2414,-0.0499)$ \\
\hline 90 & 10 & 1 & $(-0.1036,-1.2312,-0.051)$ & $(-0.1093,-1.0934,-0.051)$ & $(-0.1093,-1.0934,-0.051)$ \\
\hline 95 & 10 & 1 & $(-0.3557,-0.0449,-0.0493)$ & $(-0.3585,0.0569,-0.0491)$ & $(-0.3557,-0.0449,-0.0493)$ \\
\hline 100 & 10 & 1 & $(-0.5427,0.3123,-0.0508)$ & $(-0.5526,0.0289,-0.0503)$ & $(-0.5526,0.0289,-0.0503)$ \\
\hline 105 & 12 & 1 & $(-30.18,-32.166,-3.014)$ & $(-30.18,-32.166,-3.014)$ & $(-30.18,-32.166,-3.014)$ \\
\hline 110 & 13 & 2 & $(-7.3204,-2.0077,3.0393)$ & $(-7.3204,-2.0077,3.0393)$ & $(-7.3204,-2.0077,3.0393)$ \\
\hline 115 & 13 & 1 & $(1.4989,-1.2538,3.0388)$ & $(1.486,-1.2556,3.0388)$ & $(1.486,-1.2556,3.0388)$ \\
\hline 120 & 14 & 1 & $(-0.1376,0.1643,0.1155)$ & $(0.0158,0.1518,0.1155)$ & $(0.0158,0.1518,0.1155)$ \\
\hline 125 & 14 & 1 & $(0.9365,0.6051,0.1148)$ & $(0.8845,0.6094,0.1148)$ & $(0.8845,0.6094,0.1148)$ \\
\hline 130 & 15 & 1 & $(10.363,0.5424,0.0211)$ & $(10.3191,0.5419,0.0211)$ & $(10.3191,0.5419,0.0211)$ \\
\hline 135 & 16 & 1 & $(15.869,-14.651,-2.642)$ & $(4.3998,8.294,-14.388)$ & $(4.3998,8.294,-14.388)$ \\
\hline 140 & 17 & 6 & $(17.9441,-29.2873,-1.557)$ & $(17.92,-29.2845,-1.557)$ & $(-0.0001,-10.284,-0.1191)$ \\
\hline 145 & 19 & 1 & $(18.5319,-6.5598,-1.3091)$ & $(2.4199,-13.2322,23.564)$ & $(18.5319,-6.5598,-1.3091)$ \\
\hline 150 & 19 & 1 & $(0.0958,0.1761,0.0371)$ & $(-0.0201,0.2045,0.0563)$ & $(0.0958,0.1761,0.0371)$ \\
\hline 155 & 19 & 1 & $(-0.0123,0.1601,0.006)$ & $(-0.0488,0.1913,0.0204)$ & $(-0.0123,0.1601,0.006)$ \\
\hline 160 & 19 & 1 & $(-0.0028,0.0484,0.0023)$ & $(0.0056,0.0871,-0.0029)$ & $(-0.0028,0.0484,0.0023)$ \\
\hline 165 & 19 & 1 & $(0.1996,-0.0383,0.028)$ & $(0.1482,-0.1033,0.0276)$ & $(0.1482,-0.1033,0.0276)$ \\
\hline 170 & 19 & 1 & $(0.0644,-0.1587,0.0393)$ & $(0.1028,-0.1691,0.0301)$ & $(0.0644,-0.1587,0.0393)$ \\
\hline 175 & 19 & 1 & $(0.0334,-0.486,0.034)$ & $(0.0176,-0.3049,0.0306)$ & $(0.0176,-0.3049,0.0306)$ \\
\hline 180 & 19 & 1 & $(-0.0087,-0.0928,0.0162)$ & $(-0.0048,0.0283,0.014)$ & $(-0.0048,0.0283,0.014)$ \\
\hline 185 & 19 & 1 & $(-0.0285,-0.0564,0.0144)$ & $(-0.0395,-0.012,0.0143)$ & $(-0.0395,-0.012,0.0143)$ \\
\hline 190 & 19 & 1 & $(-0.5505,-0.0172,0.0022)$ & $(-0.5512,-0.0521,0.0115)$ & $(-0.5505,-0.0172,0.0022)$ \\
\hline
\end{tabular}


Table B-51: Error for Localisation Office Map (MCL) Case 6

\begin{tabular}{|c|c|c|c|c|c|}
\hline$\#$ & $\mathrm{R}$ & $\mathrm{C}$ & Most Likely Cluster & Most Likely Sample & Closest Cluster \\
\hline 5 & $\overline{0}$ & 2 & $(-2.4709,-8.6636,-3.1447)$ & $\overline{(-2.4379,-8.6391,-3.1448)}$ & $(-2.4379,-8.6391,-3.1448)$ \\
\hline 10 & 1 & 1 & $(19.188,15.742,-1.071)$ & $(26.971,-8.3699,7.793)$ & $(19.188,15.742,-1.071)$ \\
\hline 15 & 1 & 1 & $(29.5614,29.8784,4.3844)$ & $(29.5636,29.883,4.3844)$ & $(29.5614,29.8784,4.3844)$ \\
\hline 20 & 1 & 1 & $(24.185,33.7885,-1.6664)$ & $(24.194,33.7699,-1.666)$ & $(24.194,33.7699,-1.666)$ \\
\hline 25 & 1 & 1 & $(18.262,36.967,-1.6798)$ & $(18.278,36.94,-1.68)$ & $(18.278,36.939,-1.68)$ \\
\hline 30 & 1 & 1 & $(13.276,39.061,-1.687)$ & $(13.244,39.092,-1.709)$ & $(13.276,39.061,-1.687)$ \\
\hline 35 & 1 & 1 & $(9.5142,34.8672,-1.6651)$ & $(9.4898,34.8622,-1.6655)$ & $(9.4898,34.8622,-1.6655)$ \\
\hline 40 & 1 & 1 & $(7.5754,33.8364,4.3924)$ & $(7.5731,33.8321,4.4387)$ & $(7.5754,33.8364,4.3924)$ \\
\hline 45 & 1 & 1 & $(11.5516,39.4334,4.276)$ & $(11.7229,39.3269,4.3093)$ & $(11.7229,39.3269,4.3093)$ \\
\hline 50 & $\overline{2}$ & 5 & $(5.6737,32.3374,6.064)$ & $(5.6645,32.3358,6.064)$ & $(-0.6768,0.1832,0.2938)$ \\
\hline 55 & 2 & 1 & $(-5.6652,31.838,0.1576)$ & $(-5.7328,31.8526,0.1576)$ & $(-5.6652,31.838,0.1576)$ \\
\hline 60 & 2 & 1 & $(-6.6961,31.2624,0.162)$ & $(-6.7308,31.27,0.162)$ & $(-6.6961,31.2624,0.162)$ \\
\hline 65 & 3 & 4 & $(0.1285,38.6046,-3.047)$ & $(0.1659,38.6347,-3.0479)$ & $(-15.5566,6.3613,-1.7237)$ \\
\hline 70 & 3 & 1 & $(-19.3331,2.5109,-1.6673)$ & $(-19.2169,2.517,-1.6673)$ & $(-19.2169,2.517,-1.6673)$ \\
\hline 75 & 3 & 1 & $(-22.866,-2.246,-1.644)$ & $(-22.898,-2.248,-1.644)$ & $(-22.866,-2.246,-1.644)$ \\
\hline 80 & 3 & 1 & $(-26.3375,-6.9973,-1.64)$ & $(-26.3436,-6.9976,-1.64)$ & $(-26.3375,-6.9973,-1.64)$ \\
\hline 85 & 3 & 1 & $(-30.714,-11.787,-1.662)$ & $(-30.72,-11.787,-1.662)$ & $(-30.714,-11.787,-1.662)$ \\
\hline 90 & 4 & 15 & $(-1.8415,0.8195,-3.1452)$ & $(-2.0022,-3.4802,-3.1531)$ & $(-1.8415,0.8195,-3.1452)$ \\
\hline 95 & 4 & 1 & $(-0.626,-5.9909,-0.0798)$ & $(-0.6144,-6.1773,-0.0798)$ & $(-0.626,-5.9909,-0.0798)$ \\
\hline 100 & 4 & 1 & $(-1.0984,-6.125,-0.1064)$ & $(-1.1,-6.1223,-0.1062)$ & $(-1.1,-6.1223,-0.1062)$ \\
\hline 105 & 4 & 1 & $(-1.5085,-6.4591,-0.0881)$ & $(-1.4622,-6.4175,-0.0867)$ & $(-1.4622,-6.4175,-0.0867)$ \\
\hline 110 & 5 & 1 & $(-0.0952,0.3716,-0.0259)$ & $(-0.1122,0.3715,-0.0259)$ & $(-0.0952,0.3716,-0.0259)$ \\
\hline 115 & 5 & 1 & $(-0.2529,0.2975,-0.035)$ & $(-0.2134,0.298,-0.0349)$ & $(-0.2134,0.298,-0.0349)$ \\
\hline 120 & 5 & 1 & $(-0.6548,0.1137,-0.0522)$ & $(-0.5844,0.1119,-0.052)$ & $(-0.5844,0.1119,-0.052)$ \\
\hline 125 & 5 & 1 & $(-0.3546,-0.2119,-0.0729)$ & $(0.0413,-0.1746,-0.073)$ & $(0.0413,-0.1746,-0.073)$ \\
\hline 130 & 5 & 1 & $(0.2148,-0.6161,-0.0991)$ & $(0.3766,-0.5853,-0.0994)$ & $(0.2148,-0.6161,-0.0991)$ \\
\hline 135 & 5 & 1 & $(1.0415,-1.1201,-0.1366)$ & $(1.0864,-1.0974,-0.1358)$ & $(1.0415,-1.1201,-0.1366)$ \\
\hline 140 & 6 & 4 & $(26.079,-34.515,-1.605)$ & $(26.031,-34.516,-1.602)$ & $(8.353,-28.905,1.617)$ \\
\hline 145 & 6 & 1 & $(31.8618,-30.1921,-1.581)$ & $(31.822,-30.203,-1.580)$ & $(31.822,-30.203,-1.580)$ \\
\hline 150 & 6 & 1 & $(32.037,-24.877,4.71)$ & $(32.029,-24.907,4.714)$ & $(32.037,-24.877,4.71)$ \\
\hline 155 & 6 & 1 & $(28.478,-21.762,-1.68)$ & $(28.479,-21.761,-1.672)$ & $(28.479,-21.761,-1.672)$ \\
\hline 160 & 6 & 1 & $(23.295,-24.849,-1.764)$ & $(23.365,-24.855,-1.763)$ & $(23.295,-24.849,-1.764)$ \\
\hline 165 & 6 & 1 & $(18.619,-28.492,-1.797)$ & $(18.777,-28.533,-1.92)$ & $(18.619,-28.492,-1.797)$ \\
\hline 170 & 6 & 1 & $(19.724,-24.63,4.451)$ & $(19.665,-24.67,4.45)$ & $(19.665,-24.67,4.45)$ \\
\hline 175 & 6 & 1 & $(22.0179,-17.672,4.53)$ & $(22.0856,-17.628,4.53)$ & $(22.0179,-17.672,4.53)$ \\
\hline 180 & 7 & 1 & $(0.0774,0.4732,-0.04)$ & $(0.0758,0.4822,-0.0395)$ & $(0.0774,0.4732,-0.04)$ \\
\hline 185 & 7 & 1 & $(0.3195,-0.0918,-0.0117)$ & $(0.3361,-0.1178,-0.01)$ & $(0.3195,-0.0918,-0.0117)$ \\
\hline 190 & 7 & 1 & $(-0.2934,-0.0026,0.0384)$ & $(-0.1849,0.0298,0.0103)$ & $(-0.1849,0.0298,0.0103)$ \\
\hline
\end{tabular}




\section{B.2.2 CPF-MCL}

Table B-52: Error for Localisation Office Map (CPF-MCL) Case 1

\begin{tabular}{|c|c|c|c|c|}
\hline \# & $\mathrm{R}$ & $\mathrm{C}$ & Most Likely Cluster & Closest Cluster \\
\hline 5 & 0 & 1 & $(0.0881,-0.0428,-0.0006)$ & $(0.0881,-0.0428,-0.0006)$ \\
\hline 10 & 0 & 1 & $(0.0259,-0.1096,-0.0153)$ & $(0.0259,-0.1096,-0.0153)$ \\
\hline 15 & 0 & 1 & $(0.0775,0.0185,-0.016)$ & $(0.0775,0.0185,-0.016)$ \\
\hline 20 & 0 & 1 & $(0.5657,0.0724,0.0737)$ & $(0.5657,0.0724,0.0737)$ \\
\hline 25 & 0 & 8 & $(-3.1925,-0.0161,-0.0007)$ & $(0.8759,0.0912,0.0134)$ \\
\hline 30 & 0 & 1 & $(-0.2011,0.1897,0.0859)$ & $(-0.2011,0.1897,0.0859)$ \\
\hline 35 & 0 & 1 & $(-0.2088,0.0371,-0.0039)$ & $(-0.2088,0.0371,-0.0039)$ \\
\hline 40 & 0 & 8 & $(21.4721,-1.5806,1.5614)$ & $(0.0658,-0.0375,0.0112)$ \\
\hline 45 & 0 & 1 & $(0.0091,-0.0318,0.0069)$ & $(0.0091,-0.0318,0.0069)$ \\
\hline 50 & 0 & 2 & $(0.1006,0.0197,-3.6225)$ & $(0.1006,0.0197,-3.6225)$ \\
\hline 55 & 0 & 7 & $(0.5853,-0.0008,-6.2678)$ & $(-2.3112,-0.8417,-3.1376)$ \\
\hline 60 & 0 & 2 & $(0.0705,-0.0011,0.0011)$ & $(0.0705,-0.0011,0.0011)$ \\
\hline 65 & 0 & 6 & $(-0.1992,0.4776,0.0763)$ & $(-0.1992,0.4776,0.0763)$ \\
\hline 70 & 0 & 1 & $(-11.5699,13.9735,-4.7056)$ & $(-11.5699,13.9735,-4.7056)$ \\
\hline 75 & 0 & 1 & $(-11.6083,9.4662,-4.6512)$ & $(-11.6083,9.4662,-4.6512)$ \\
\hline 80 & 0 & 3 & $(0.1032,1.0101,-0.0236)$ & $(0.1032,1.0101,-0.0236)$ \\
\hline 85 & 0 & 1 & $(-0.0176,1.4983,-0.0245)$ & $(-0.0176,1.4983,-0.0245)$ \\
\hline 90 & 0 & 5 & $(-0.5092,-4.6344,-3.127)$ & $(0.0389,0.3103,0.0294)$ \\
\hline 95 & 0 & 1 & $(0.2186,0.3117,0.0325)$ & $(0.2186,0.3117,0.0325)$ \\
\hline 100 & 0 & 2 & $(-1.1454,-20.1043,-3.0673)$ & $(-1.1454,-20.1043,-3.0673)$ \\
\hline 105 & 0 & 1 & $(-0.0129,-6.9726,2.901)$ & $(-0.0129,-6.9726,2.901)$ \\
\hline 110 & 0 & 5 & $(0.1667,-0.0458,0.062)$ & $(0.1667,-0.0458,0.062)$ \\
\hline 115 & $\begin{array}{lll}0 \\
0\end{array}$ & 3 & $(-0.1466,0.1539,0.0484)$ & $(-0.1466,0.1539,0.0484)$ \\
\hline 120 & 0 & 2 & $(0.0811,0.371,0.047)$ & $(0.0811,0.371,0.047)$ \\
\hline 125 & 0 & 1 & $(-0.7543,0.1544,0.0347)$ & $(-0.7543,0.1544,0.0347)$ \\
\hline 130 & 0 & 2 & $(9.9471,0.0658,0.103)$ & $(0.5551,0.0114,-0.0933)$ \\
\hline 135 & $\begin{array}{llll}0 & \\
\end{array}$ & 3 & $(0.1216,-18.8728,0.4595)$ & $(0.1475,-0.247,0.0936)$ \\
\hline 140 & 0 & 1 & $(0.092,-0.6025,0.0337)$ & $(0.092,-0.6025,0.0337)$ \\
\hline 145 & $\begin{array}{ll}0 \\
0\end{array}$ & 1 & $(0.0077,-0.43,-0.0387)$ & $(0.0077,-0.43,-0.0387)$ \\
\hline 150 & $\begin{array}{ll}0 \\
0\end{array}$ & 1 & $(0.3605,0.339,0.0358)$ & $(0.3605,0.339,0.0358)$ \\
\hline 155 & 0 & 4 & $(-0.0408,0.3069,0.0145)$ & $(-0.0408,0.3069,0.0145)$ \\
\hline 160 & $\mathbf{0}$ & 3 & $(-0.0335,0.0592,0.0087)$ & $(-0.0335,0.0592,0.0087)$ \\
\hline 165 & $\begin{array}{ll}0 & 0 \\
\end{array}$ & 6 & $(0.1948,-0.0323,0.0277)$ & $(0.1948,-0.0323,0.0277)$ \\
\hline 170 & $\begin{array}{c}0 \\
0\end{array}$ & 3 & $(0.0711,-0.1947,0.0409)$ & $(0.0711,-0.1947,0.0409)$ \\
\hline 175 & 0 & 8 & $(-0,4.5949,0.0235)$ & $(0.032,-0.5209,0.0357)$ \\
\hline 180 & 0 & $\overline{3}$ & $(-0.2815,5.3799,0.0463)$ & $(0.0781,-1.0361,0.002)$ \\
\hline 185 & 0 & 1 & $(-0.2071,-0.1825,0.0023)$ & $(-0.2071,-0.1825,0.0023)$ \\
\hline 190 & 0 & 5 & $(-0.756,0.0064,-0.0057)$ & $(-0.756,0.0064,-0.0057)$ \\
\hline
\end{tabular}


Table B-53: Error for Localisation Office Map (CPF-MCL) Case 2

\begin{tabular}{|c|c|c|c|c|}
\hline$\#$ & $\mathrm{R}$ & $y$ & r & er \\
\hline 5 & 0 & & $.0489,24.1002,0.0239)$ & $(0.007,0.09$ \\
\hline 10 & 0 & 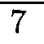 & $(7.5408,2.1006,4.7946)$ & $(0.2581,-0.1069,-0.0202)$ \\
\hline 15 & 0 & 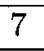 & $(0.2777,-0.1792,-0.0169)$ & $(0.2777,-0.1792,-0.0169)$ \\
\hline 20 & 0 & & $1134,0.0444)$ & $(0.9217,0.1134,0.0444)$ \\
\hline 25 & 0 & f & $247,0.0274)$ & $(1.228,0.1247,0.0274)$ \\
\hline 30 & 8 & $\tau$ & $(1.4$ & $(1.4546,0.2351,0.0312)$ \\
\hline 35 & 0 & 7 & $3171,-0.0076)$ & $(-11.0784,9.7205,-2.67)$ \\
\hline 40 & 0 & $\overline{5}$ & 1082,0.0077) & $(-12.2643,7.3382,4.0038)$ \\
\hline 45 & 0 & 8 & $5,-0.0312)$ & $(0.0229,0.2045,-0.0312)$ \\
\hline 50 & 0 & 5 & $1.2919)$ & $(0.4304,0.1103,1.2919)$ \\
\hline 55 & 0 & 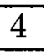 & $,-0.0055)$ & $(0.8624,0.0438,-0.0055)$ \\
\hline 60 & 0 & $r$ & $(-3.2$ & $\overline{(0.6569,0}$ \\
\hline 65 & 0 & & $(0.3$ & $(0.3924,0$ \\
\hline 70 & 0 & 0 & $(0.0$ & $(-0.07$ \\
\hline 75 & 0 & 0 & $(-0)$. & $(-0.0817,0.5406,-0.0088)$ \\
\hline 80 & 0 & 5 & $(-0$. & $(-0.1$ \\
\hline 85 & 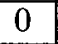 & 0 & $(-0$. & $(-0.20$ \\
\hline 90 & 0 & 5 & $(-0$. & $(-0.00$ \\
\hline 95 & 0 & 4 & & $(-0.1)$ \\
\hline 100 & 0 & 7 & & $(0.1$ \\
\hline 105 & 0 & 7 & $(-0$ & $(-0.3$ \\
\hline 110 & 0 & 6 & $(-21$ & $(-0.41$ \\
\hline 115 & 0 & 6 & $(0.1$ & $(0.1057$ \\
\hline 120 & 0 & 8 & & $(-0.03$ \\
\hline 125 & 0 & 6 & $(-0.8)$ & $(0.70$ \\
\hline 130 & 0 & 5 & $(-4.30$ & $(1.498$ \\
\hline 135 & 0 & 6 & 777) & $(-2.5721,-2.1852,-0.217)$ \\
\hline 140 & 0 & 4 & (30. & $27,-0.2135)$ \\
\hline 145 & 0 & 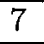 & & $11,-0.0657)$ \\
\hline 150 & 0 & 5 & & 334) \\
\hline 155 & 0 & 7 & $(2.7$ & $(0.1297,0.1364,-0.0012)$ \\
\hline 160 & 0 & 5 & & $(0.0926,0.3829,-0.0083)$ \\
\hline 165 & 0 & 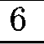 & & $(0.2513,0.1019,0.0006)$ \\
\hline 170 & 0 & 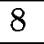 & $(0.1$ & $(0.1531,-0.239,0.0592)$ \\
\hline 175 & 0 & 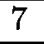 & $(-0.0$ & $(-0.01$ \\
\hline 180 & 0 & 0 & $(6.9$ & $(-0.2631,-0$. \\
\hline 185 & 0 & 8 & $(23.8$ & $.1648)$ \\
\hline 190 & $\overline{0}$ & 6 & $(-1.9428,-0.5732,0.2028)$ & $(-1.9428,-0.5732,0.2028)$ \\
\hline
\end{tabular}


Table B-54: Error for Localisation Office Map (CPF-MCL) Case 3

\begin{tabular}{|c|c|c|c|c|}
\hline$\#$ & $\mathrm{R}$ & $\mathrm{C}$ & Most Likely Cluster & Closest Cluster \\
\hline$\overline{5}$ & 0 & $\overline{1}$ & $(21.4847,15.1419,-5.7478)$ & 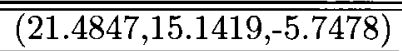 \\
\hline 10 & 0 & 1 & $(0.1876,0.0133,0.0317)$ & $(0.1876,0.0133,0.0317)$ \\
\hline 15 & 0 & 1 & $(0.0955,-0.0075,0.0311)$ & $(0.0955,-0.0075,0.0311)$ \\
\hline 20 & 0 & 7 & $(6.3257,0.1012,0.0314)$ & $(0.6135,0.0631,0.0681)$ \\
\hline 25 & 0 & 3 & $(2.1011,0.0794,0.0563)$ & $(2.1011,0.0794,0.0563)$ \\
\hline 30 & 0 & 1 & $(-15.1011,25.7968,-1.6388)$ & $(-15.1011,25.7968,-1.6388)$ \\
\hline 35 & 0 & 1 & $(-9.9897,1.5215,-3.131)$ & $(-9.9897,1.5215,-3.131)$ \\
\hline 40 & 0 & 1 & $(-0.1457,-0.2723,-0.0246)$ & $(-0.1457,-0.2723,-0.0246)$ \\
\hline 45 & 0 & 1 & $(-0.0904,0.0462,-0.0094)$ & $(-0.0904,0.0462,-0.0094)$ \\
\hline 50 & 0 & 8 & $(-7.3393,-0.8326,-3.1561)$ & $(0.0923,0.0186,-2.8532)$ \\
\hline 55 & 0 & 6 & $(0.6111,-0.0025,-4.5157)$ & $(-3.001,-0.7959,-3.1119)$ \\
\hline 60 & 0 & 3 & $(0.053,-0.0031,-0.0018)$ & $(0.053,-0.0031,-0.0018)$ \\
\hline 65 & 0 & 1 & $(-0.1826,0.5009,0.0629)$ & $(-0.1826,0.5009,0.0629)$ \\
\hline 70 & 0 & 8 & $(0.0054,1.7642,-0.0078)$ & $(-0.0491,0.271,0.026)$ \\
\hline 75 & 0 & 1 & $(0.0659,0.0751,0.0267)$ & $(0.0659,0.0751,0.0267)$ \\
\hline 80 & 0 & 2 & $(0.1946,0.1979,0.0278)$ & $(0.1946,0.1979,0.0278)$ \\
\hline 85 & 0 & 7 & $(-0.0028,4.4458,0.0055)$ & $(0.2996,-0.3093,0.027)$ \\
\hline 90 & 0 & 3 & $(-0.3937,-5.6662,-3.1691)$ & $(0.4489,0.5892,0.0819)$ \\
\hline 95 & 0 & 1 & $(-11.8485,-8.9781,-4.9196)$ & $(-11.8485,-8.9781,-4.9196)$ \\
\hline 100 & 0 & 2 & $(0.1628,0.4103,0.0112)$ & $(0.1628,0.4103,0.0112)$ \\
\hline 105 & 0 & 1 & $(-0.1481,-0.0735,0.0391)$ & $(-0.1481,-0.0735,0.0391)$ \\
\hline 110 & 0 & 2 & $(0.0061,0.0221,0.0229)$ & $(0.0061,0.0221,0.0229)$ \\
\hline 115 & 0 & 1 & $(-0.2233,0.1036,0.0188)$ & $(-0.2233,0.1036,0.0188)$ \\
\hline 120 & 0 & 1 & $(-0.6625,0.1783,0.0171)$ & $(-0.6625,0.1783,0.0171)$ \\
\hline 125 & 0 & 1 & $(-0.582,0.256,0.0164)$ & $(-0.582,0.256,0.0164)$ \\
\hline 130 & 0 & 1 & $(9.689,-0.0116,-0.0572)$ & $(9.689,-0.0116,-0.0572)$ \\
\hline 135 & 0 & 5 & $(11.8166,-29.2637,1.5868)$ & $(-1.9884,-8.9629,-1.5511)$ \\
\hline 140 & 0 & 1 & $(16.1872,-28.9765,1.6004)$ & $(16.1872,-28.9765,1.6004)$ \\
\hline 145 & 0 & 5 & $(-0.0488,-7.0384,-0.0387)$ & $(-0.0573,0.4057,0.0708)$ \\
\hline 150 & 0 & 2 & $(0.391,-0.5471,0.0165)$ & $(0.391,-0.5471,0.0165)$ \\
\hline 155 & 0 & 1 & $(0.1086,0.2929,-0.013)$ & $(0.1086,0.2929,-0.013)$ \\
\hline 160 & 0 & 1 & $(-0.0031,0.0663,-0.0078)$ & $(-0.0031,0.0663,-0.0078)$ \\
\hline 165 & 0 & 2 & $(0.1538,-0.045,0.0205)$ & $(0.1538,-0.045,0.0205)$ \\
\hline 170 & 0 & 1 & $(0.057,-0.2161,0.0331)$ & $(0.057,-0.2161,0.0331)$ \\
\hline 175 & 0 & 6 & $(0.0295,-0.5452,0.0311)$ & $(0.0295,-0.5452,0.0311)$ \\
\hline 180 & 0 & 5 & $(-0.025,-0.1187,0.0165)$ & $(-0.025,-0.1187,0.0165)$ \\
\hline 185 & 0 & 5 & $(24.5254,-7.2823,1.5563)$ & $(-0.0952,-0.0776,0.0166)$ \\
\hline 190 & 0 & 4 & $(-0.6355,-0.0275,0.0049)$ & $(-0.6355,-0.0275,0.0049)$ \\
\hline
\end{tabular}


Table B-55: Error for Localisation Office Map (CPF-MCL) Case 4

\begin{tabular}{|c|c|c|c|c|}
\hline \# & $\mathrm{R}$ & C & $\mathrm{M}$ & ister \\
\hline$\overline{5}$ & $\overline{00}$ & 8 & 5) & $\overline{(-2.2}$ \\
\hline 10 & 0 & 8 & $(31.8516,8.3886,3.2738)$ & $(-0.0664,-0.3761,-0.0547)$ \\
\hline 15 & 0 & 7 & $(0.1212,-0.1708,-0.0658)$ & $(0.1212,-0.1708,-0.0658)$ \\
\hline 20 & 0 & 5 & $(0.7658,0.1213,0.0434)$ & $(0.7658,0.1213,0.0434)$ \\
\hline 25 & 0 & 7 & $(1.1965,0.1166,0.0248)$ & $(1.1965,0.1166,0.0248)$ \\
\hline 30 & 0 & 6 & $(0.1203,0.1909,-0.0323)$ & $(0.1203,0.1909,-0.0323)$ \\
\hline 35 & 0 & 6 & $4,0.2157,-0.0289)$ & $(-0.1524,0.2157$ \\
\hline 40 & 0 & 6 & $(0.1127,0.11$ & $(0.1127,0.1)$ \\
\hline 45 & 0 & 6 & $(-0.0333,-0.0442,0.0097)$ & $(-0.0333,-0.0442,0.0097)$ \\
\hline 50 & 0 & 8 & $2293)$ & $\overline{(0.3839,0.0474,2.2293)}$ \\
\hline 55 & 0 & 6 & $2858)$ & $2858)$ \\
\hline 60 & 0 & 4 & $(0.7311,0.0045,-0.0168)$ & $(0.7311,0.0045,-0.0168)$ \\
\hline 65 & 0 & 7 & $(0.2011,0.5832,-0.0006)$ & $(0.2011,0.5832,-0.0006)$ \\
\hline 70 & 0 & 7 & $(-24.5578,-3.8082,1.4194)$ & $(0.716,0.4209,0.1966)$ \\
\hline 75 & 0 & 5 & $(-19.7082,-7.7856,1.4325)$ & $(1.5901,0.11$ \\
\hline 80 & 0 & 6 & 919) & $(-0.099$ \\
\hline 85 & 0 & 6 & $5,0.5634$ & $(0.2955,0.5634,0.0703)$ \\
\hline 90 & 0 & 8 & $(-0.0397,-5.127$ & $(0.5839,0.0827,0.0564)$ \\
\hline 95 & 0 & 4 & $(0.9479,0.18$ & $56,0.0925)$ \\
\hline 100 & 0 & 4 & $(-1.4072,-3.2201,-0.1323)$ & $(1.1966,-0.4252,0.0657)$ \\
\hline 105 & 0 & 8 & $(0.7344,-0.0411,-0.0125)$ & $(0.7344,-0.0411,-0.0125)$ \\
\hline 110 & 0 & 8 & $(-0.212,-0.2837,-0.0733)$ & $(-0.212,-0.2837,-0.0733)$ \\
\hline 115 & 0 & 6 & $(0.1049,-0.4912,-0.0755)$ & $(0.1049,-0.4912,-0.0755)$ \\
\hline 120 & 0 & 5 & $(-1.7729,-$ & $(-0.1878,-0.8402,-0.0935)$ \\
\hline 125 & 0 & 8 & $0.0637)$ & $(1.2117,0.0028,-0.0507)$ \\
\hline 130 & 0 & 7 & $\overline{(1.4}$ & $718,-0.044)$ \\
\hline 135 & 0 & 6 & $(9.2$ & $(0.51,-0.7184,-0.0787)$ \\
\hline 140 & 0 & 4 & $, 0.0659)$ & $(0.6203,-1.6024,0.0586)$ \\
\hline 145 & 0 & 4 & $(26.8184,-3$ & $(0.3981,-1.1682,0.0836)$ \\
\hline 150 & 0 & 4 & $(0.3757,-0.3002,-0.0375)$ & $(0.3757,-0.3002,-0.0375)$ \\
\hline 155 & 0 & 4 & $(0.3249,0.1447,-0.0581)$ & $(0.3249,0.1447,-0.0581)$ \\
\hline 160 & 0 & 6 & $(0.1383,0.4196,-0.0361)$ & $(0.1383,0.4196,-0.0361)$ \\
\hline 165 & 0 & 6 & $(0.1837,0.101,-0.0201)$ & $(0.1837,0.101,-0.0201)$ \\
\hline 170 & 0 & 8 & $(-0.188,-4.2318,-0.1397)$ & $(0.1302,-0.2434,0.0482)$ \\
\hline 175 & 0 & 8 & $(-0.0033,-0.9535,0.1202)$ & $(-0.0033,-0.9535,0.1202)$ \\
\hline 180 & 0 & 6 & $(-0.2626,-0.508,0.1257)$ & $(-0.2626,-0.508,0.1257)$ \\
\hline 185 & 0 & 5 & $(-0.9489,-\mathrm{C}$ & $(-0.9489,-0.8122,0.1532)$ \\
\hline 190 & 0 & 8 & $(10.614,3.36$ & $(0.8$ \\
\hline
\end{tabular}


Table B-56: Error for Localisation Office Map (CPF-MCL) Case 5

\begin{tabular}{|c|c|c|c|c|}
\hline$\#$ & $\mathrm{R}$ & $\mathrm{C}$ & Most Likely Cluster & Closest Cluster \\
\hline 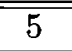 & 0 & 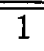 & 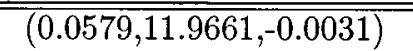 & $\overline{(0.0579,11.9661,-0.0031)}$ \\
\hline 10 & 0 & 1 & $(29.517,25.2752,0.082)$ & $(29.517,25.2752,0.082)$ \\
\hline 15 & 0 & 1 & $(31.9121,35.3538,2.3825)$ & $(31.9121,35.3538,2.3825)$ \\
\hline 20 & 0 & 5 & $(15.9012,1.1887,-3.1297)$ & $(1.2296,0.0978,0.0358)$ \\
\hline 25 & 0 & 4 & $(2.706,0.2022,0.0344)$ & $(2.706,0.2022,0.0344)$ \\
\hline 30 & 0 & 1 & $(-15.2778,14.2641,-1.6922)$ & $(-15.2778,14.2641,-1.6922)$ \\
\hline 35 & 0 & 2 & $(23.983,-5.9238,0.1035)$ & $(23.983,-5.9238,0.1035)$ \\
\hline 40 & $\begin{array}{l}0 \\
\end{array}$ & 1 & $(3.4536,11.3446,-1.077)$ & $(3.4536,11.3446,-1.077)$ \\
\hline 45 & 0 & 1 & $(-13.9735,-3.9218,0.3933)$ & $(-13.9735,-3.9218,0.3933)$ \\
\hline 50 & 0 & 1 & $(17.1386,-0.8561,-3.116)$ & $(17.1386,-0.8561,-3.116)$ \\
\hline 55 & 0 & 7 & $(-9.2182,-0.0322,-0.049)$ & $(-0.1376,-0.0047,-0.1063)$ \\
\hline 60 & 0 & 3 & $(-0.2033,0.4462,6.1766)$ & $(-0.2033,0.4462,6.1766)$ \\
\hline 65 & 0 & 1 & $(-0.5371,24.8437,-5.2298)$ & $(-0.5371,24.8437,-5.2298)$ \\
\hline 70 & 0 & 1 & $(-0.3245,21.0003,-3.1073)$ & $(-0.3245,21.0003,-3.1073)$ \\
\hline 75 & 0 & 1 & $(-11.6079,9.5039,-4.4127)$ & $(-11.6079,9.5039,-4.4127)$ \\
\hline 80 & 0 & 2 & $(-0.3362,4.5748,-3.0317)$ & $(-0.3362,4.5748,-3.0317)$ \\
\hline 85 & 0 & 1 & $(-0.2935,-2.801,-3.1087)$ & $(-0.2935,-2.801,-3.1087)$ \\
\hline 90 & 0 & 1 & $(-0.5615,2.1328,-3.1027)$ & $(-0.5615,2.1328,-3.1027)$ \\
\hline 95 & 0 & 1 & $(-11.4794,-9.1586,-4.3698)$ & $(-11.4794,-9.1586,-4.3698)$ \\
\hline 100 & 0 & 1 & $(-2.8475,-20.7402,-4.4246)$ & $(-2.8475,-20.7402,-4.4246)$ \\
\hline 105 & 0 & 2 & $(-30.1326,-12.54,-1.833)$ & $(-30.1326,-12.54,-1.833)$ \\
\hline 110 & 0 & 2 & $(-22.566,-0.1505,0.0032)$ & $(-18.0685,-1.9517,-3.0797)$ \\
\hline 115 & 0 & 1 & $(-2.4444,-15.3712,-2.3624)$ & $(-2.4444,-15.3712,-2.3624)$ \\
\hline 120 & 0 & 1 & $(-5.4792,-0.1861,-0.149)$ & $(-5.4792,-0.1861,-0.149)$ \\
\hline 125 & 0 & 1 & $(6.8725,-15.0871,-3.1132)$ & $(6.8725,-15.0871,-3.1132)$ \\
\hline 130 & 0 & 1 & $(10.2303,0.0232,-0.1436)$ & $(10.2303,0.0232,-0.1436)$ \\
\hline 135 & 0 & 1 & $(-4.4287,-16.9133,3.4537)$ & $(-4.4287,-16.9133,3.4537)$ \\
\hline 140 & 0 & 1 & $(17.9536,-11.7837,-1.4683)$ & $(17.9536,-11.7837,-1.4683)$ \\
\hline 145 & 0 & 3 & $(21.5733,8.0477,2.3304)$ & $(8.1591,7.7507,4.6144)$ \\
\hline 150 & 0 & 1 & $(29.4314,-11.086,1.3011)$ & $(29.4314,-11.086,1.3011)$ \\
\hline 155 & 0 & 2 & $(0.3745,0.0142,-0.0768)$ & $(0.3745,0.0142,-0.0768)$ \\
\hline 160 & 0 & 2 & $(0.1419,0.0329,-0.0606)$ & $(0.1419,0.0329,-0.0606)$ \\
\hline 165 & 0 & 3 & $(26.2946,-11.1314,-1.8211)$ & $(0.1079,-0.0449,0.0133)$ \\
\hline 170 & 0 & 2 & $(0.0556,-0.2253,0.0294)$ & $(0.0556,-0.2253,0.0294)$ \\
\hline 175 & 0 & 3 & $(0.0294,-0.4951,0.028)$ & $(0.0294,-0.4951,0.028)$ \\
\hline 180 & 0 & 2 & $(0.0276,-0.0367,0.0068)$ & $(0.0276,-0.0367,0.0068)$ \\
\hline 185 & 0 & 2 & $(0.0247,-0.0329,0.0061)$ & $(0.0247,-0.0329,0.0061)$ \\
\hline 190 & 0 & 3 & $(-0.4725,-0.0049,-0.0021)$ & $(-0.4725,-0.0049,-0.0021)$ \\
\hline
\end{tabular}


Table B-57: Error for Localisation Office Map (CPF-MCL) Case 6

\begin{tabular}{|c|c|c|c|c|}
\hline$\#$ & $\mathrm{R}$ & $\mathrm{C}$ & Most Likely Cluster & Closest Cluster \\
\hline 5 & 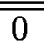 & 8 & $(-0.6579,0.1673,-0.4133)$ & $(-0.6579,0.1673,-0.4133)$ \\
\hline 10 & 0 & 6 & $(0.347,17.5845,0.0794)$ & $(-0.2527,-0.0235,-0.2058)$ \\
\hline 15 & 0 & 6 & $(-0.0477,-1.2877,-0.7458)$ & $(0.776,0.4985,-0.2288)$ \\
\hline 20 & 0 & 8 & $(14.5265,1.3991,-3.1567)$ & $(0.4746,1.072,-0.0498)$ \\
\hline 25 & 0 & 7 & $(5.3687,-0.8554,-3.0772)$ & $(-0.4386,1.1227,-0.063)$ \\
\hline 30 & 0 & 6 & $(-2.5336,-2.1747,-3.0797)$ & $(-0.6999,-0.7138,0.0532)$ \\
\hline 35 & 0 & 7 & $(-0.5657,0.125,-0.0526)$ & $(-0.5657,0.125,-0.0526)$ \\
\hline 40 & 0 & 6 & $(-0.3172,0.086,-0.0685)$ & $(-0.3172,0.086,-0.0685)$ \\
\hline 45 & 0 & 6 & $(-12.6731,-1.6946,1.6736)$ & $(-0.164,0.0528,-0.0497)$ \\
\hline 50 & 0 & 4 & $(0.2288,0.0737,1.514)$ & $(0.2288,0.0737,1.514)$ \\
\hline 55 & 0 & 8 & $(0.728,0.0438,0.1449)$ & $(0.728,0.0438,0.1449)$ \\
\hline 60 & 0 & 7 & $(0.6823,-0.0155,-0.012)$ & $(0.6823,-0.0155,-0.012)$ \\
\hline 65 & 0 & 6 & $(0.1709,0.5395,0.0084)$ & $(0.1709,0.5395,0.0084)$ \\
\hline 70 & 0 & 7 & $(-0.1307,20.5823,-3.1502)$ & $(0.4621,0.1645,0.1109)$ \\
\hline 75 & 0 & 4 & $(0.9836,0.0519,0.134)$ & $(0.9836,0.0519,0.134)$ \\
\hline 80 & 0 & 8 & $(0.1354,0.9403,-0.1152)$ & $(0.1354,0.9403,-0.1152)$ \\
\hline 85 & 0 & 7 & $(-1.5604,6.6914,-3.1078)$ & $(-0.0564,1.4182,0.0331)$ \\
\hline 90 & 0 & 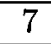 & $(0.1402,1.8017,0.0193)$ & $(0.1402,1.8017,0.0193)$ \\
\hline 95 & 0 & 7 & $(-29.9269,4.9907,0.0199)$ & $(0.3376,2.0188,0.0554)$ \\
\hline 100 & 0 & 5 & $(-2.8395,-20.2266,-3.1121)$ & $(0.4557,1.6817,0.0288)$ \\
\hline 105 & 0 & 8 & $(-29.3741,-18.6168,-1.5297)$ & $(0.7092,0.1403,0.0408)$ \\
\hline 110 & 0 & 6 & $(-0.0438,0.1734,-0.018)$ & $(-0.0438,0.1734,-0.018)$ \\
\hline 115 & 0 & 5 & $(-0.21,0.1369,-0.0272)$ & $(-0.21,0.1369,-0.0272)$ \\
\hline$\overline{120}$ & 0 & 8 & $(-0.62,-0.0081,-0.0443)$ & $(-0.62,-0.0081,-0.0443)$ \\
\hline 125 & 0 & 8 & $(-5.4604,0.28,0.0662)$ & $(-0.9584,-0.3566,-0.0651)$ \\
\hline 130 & 0 & 5 & $(-0.4453,-0.7252,-0.0911)$ & $(-0.4453,-0.7252,-0.0911)$ \\
\hline 135 & 0 & 5 & $(-5.8407,0.4186,0.0119)$ & $(-0.2562,-1.5333,-0.1283)$ \\
\hline 140 & 0 & 7 & $(0.1137,-18.7566,0.1204)$ & $(0.2557,-2.9733,-0.2212)$ \\
\hline 145 & 0 & 7 & $(-0.3849,-18.5313,0.1403)$ & $(1.3568,-2.7429,-0.2113)$ \\
\hline 150 & 0 & 8 & $(39.9623,-1.976,5.2611)$ & $(2.0744,-1.5514,5.9947)$ \\
\hline 155 & 0 & 2 & $(1.7371,-0.38,-0.3099)$ & $(1.7371,-0.38,-0.3099)$ \\
\hline 160 & 0 & 3 & $(0.5729,0.1239,-0.2986)$ & $(0.5729,0.1239,-0.2986)$ \\
\hline 165 & 0 & 7 & $(7.3836,-4.3025,-1.6234)$ & $(-0.2603,0.0593,-0.2231)$ \\
\hline 170 & 0 & 6 & $(-0.0006,-0.2662,-0.0314)$ & $(-0.0006,-0.2662,-0.0314)$ \\
\hline 175 & 0 & 4 & $(-0.009,-0.7036,0.0725)$ & $(-0.009,-0.7036,0.0725)$ \\
\hline 180 & 0 & 4 & $(0.1816,-0.4705,0.0648)$ & $(0.1816,-0.4705,0.0648)$ \\
\hline 185 & 0 & 6 & $(-4.9351,13.4332,4.6368)$ & $(-0.235,-0.6665,0.0926)$ \\
\hline 190 & 0 & 6 & $(-1.1153,-0.3614,0.1281)$ & $(-1.1153,-0.3614,0.1281)$ \\
\hline
\end{tabular}




\section{B.2.3 UCPF-MCL}

Table B-58: Error for Localisation Office Map (UCPF-MCL) Case 1

\begin{tabular}{|c|c|c|c|c|}
\hline \# & $\mathrm{R}$ & $\mathrm{C}$ & Most Likely Cluster & Closest Cluster \\
\hline 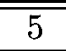 & $\overline{0}$ & $\overline{77}$ & $(49.4543,43.5791,-0.3354)$ & $\overline{(25.5291,42.5851,-1.0761)}$ \\
\hline 10 & 1 & 1 & $(0.0844,0.6947,5.8173)$ & $(0.0844,0.6947,5.8173)$ \\
\hline 15 & 2 & 3 & $(0.3654,0.2203,0.0801)$ & $(0.3654,0.2203,0.0801)$ \\
\hline 20 & 2 & 2 & $(24.6491,-3.9296,-3.0946)$ & $(0.8615,0.051,0.0776)$ \\
\hline 25 & 2 & 2 & $(0.8412,0.0559,0.0059)$ & $(0.8412,0.0559,0.0059)$ \\
\hline 30 & 2 & 2 & $(-0.0727,0.0364,0.0117)$ & $(-0.0727,0.0364,0.0117)$ \\
\hline 35 & 2 & $\overline{1}$ & $(-0.1349,0.0734,-0.0145)$ & $(-0.1349,0.0734,-0.0145)$ \\
\hline 40 & 2 & 1 & 0395) & $(0.0785,-0.0688$ \\
\hline 45 & 2 & $\overline{1}$ & $-0.0053,0.0013)$ & $(0.0639,-0.0053$ \\
\hline 50 & 2 & 1 & $(-0.2195,0.0286,-2.2815)$ & $(-0.2195,0.0286,-2.2815)$ \\
\hline 55 & 2 & 1 & $(-0.3276,0.069,-2.1956)$ & $(-0.3276,0.069,-2.1956)$ \\
\hline 60 & 2 & 1 & $(-0$. & $(-0.0287,0.15$ \\
\hline 65 & 2 & $\overline{1}$ & $\left(-0 . a^{2}\right.$ & $(-0.3375,0.3883,0.1004)$ \\
\hline 70 & $\overline{2}$ & 1 & $(-0.17$ & $(-0.1766,0.0261,-0.0171)$ \\
\hline 75 & 2 & 1 & $(-0.0$ & $(-0.0797,0.1972,0.0166)$ \\
\hline 80 & 2 & 1 & & $(0.01$ \\
\hline 85 & 2 & 1 & & $(0.0$ \\
\hline 90 & 2 & 1 & $(0.1$ & $(0.1525,0.7468,0$ \\
\hline 95 & 2 & $\overline{1}$ & $(0.21)$ & $(0.2106,0.1383,-0.0033)$ \\
\hline 100 & 2 & 1 & $(0.1$ & $(0.1818,0.119,-0.0101)$ \\
\hline 105 & $\overline{2}$ & $\overline{1}$ & $(-0.0$ & $73,0.0072)$ \\
\hline 110 & 2 & 1 & $(-0$. & $(-0.0583$ \\
\hline 115 & 2 & $\overline{1}$ & $(0.0$ & $(0.0544,0.0536,0.0096)$ \\
\hline 120 & 2 & 1 & $(-0.4$ & $(-0.451,0.0842,0.0084)$ \\
\hline 125 & 2 & 1 & $(-0.262$ & $(-0.262$ \\
\hline 130 & 2 & 1 & $(-0.2576$ & $(-0.2576$ \\
\hline 135 & 2 & 1 & $(0.0$ & $(0.0599$, \\
\hline 140 & 2 & 1 & $(0.134$ & $(0.1346,-0.2879,0.0507)$ \\
\hline 145 & $\overline{2}$ & 1 & $0525,0.0384)$ & $(-0.0075,0.0525,0.0384)$ \\
\hline 150 & 2 & 1 & $(0.4252,0.0713,0.0936)$ & $(0.4252,0.0713,0.0936)$ \\
\hline 155 & $\overline{2}$ & 1 & $(-0.042,0.0549,0.0001)$ & $(-0.042,0.0549,0.0001)$ \\
\hline 160 & 2 & 1 & $(-0.0308,0.0315,0.0048)$ & $(-0.0308,0.0315,0.0048)$ \\
\hline 165 & 2 & 1 & $(0.1795,-0.0312,0.0222)$ & $(0.1795,-0.0312,0.0222)$ \\
\hline 170 & 2 & 1 & $(0.1024,-0$. & $(0.1024,-0.3016,0.0405)$ \\
\hline 175 & 2 & 1 & $(-0.0459,-0.522,0.0476)$ & $(-0.0459,-0.522,0.0476)$ \\
\hline 180 & 2 & 1 & $(-0.1153,-0.2126,0.0172)$ & $(-0.1153,-0.2126,0.0172)$ \\
\hline 185 & 2 & $\overline{1}$ & $(-0.2012,-0.0446,0.0127)$ & $(-0.2012,-0.0446,0.0127)$ \\
\hline 190 & 2 & 1 & $(-0.449,0.0803,-0.0512)$ & $(-0.449,0.0803,-0.0512)$ \\
\hline
\end{tabular}


Table B-59: Error for Localisation Office Map (UCPF-MCL) Case 2

\begin{tabular}{|c|c|c|c|c|}
\hline$\#$ & $\mathrm{R}$ & $\mathrm{C}$ & Most Likely Cluster & Closest Cluster \\
\hline 5 & $\overline{0}$ & 8 & $(-0.1874,18.1029,0.0568)$ & $\overline{(-0.1324,0.0191,-0.0795)}$ \\
\hline 10 & 0 & 2 & $(0.0651,-0.0908,-0.0114)$ & $(0.0651,-0.0908,-0.0114)$ \\
\hline 15 & 0 & 1 & $(0.1753,-0.0763,-0.0255)$ & $(0.1753,-0.0763,-0.0255)$ \\
\hline 20 & 0 & 1 & $17,-0.0016,0.019)$ & $(0.8117,-0.0016,0.019)$ \\
\hline 25 & 0 & 1 & $4,0.0179)$ & $(1.234,0.0334,0.0179)$ \\
\hline 30 & 0 & 1 & $5,-0.0909)$ & $(0.061,-0.05,-0.0909)$ \\
\hline 35 & 0 & 1 & $685,-0.0252)$ & $(0.0934,0.2685,-0.0252)$ \\
\hline 40 & 0 & 1 & $11,-0.0114)$ & $(-0.0255,0.0311,-0.0114)$ \\
\hline 45 & 0 & 1 & $(-0.0509,0.1266,-0.0181)$ & $(-0.0509,0.1266,-0.0181)$ \\
\hline 50 & 0 & 1 & $(0.2736,0.0111,0.4645)$ & $(0.2736,0.0111,0.4645)$ \\
\hline 55 & 0 & 1 & $(-0.0007,-0.0294,3.9404)$ & $(-0.0007,-0.0294,3.9404)$ \\
\hline 60 & 0 & 1 & $(0.2305,0.0638,-0.0244)$ & $(0.2305,0.0638,-0.0244)$ \\
\hline 65 & 0 & 1 & $(-1.6547,-0.0534,0.4389)$ & $(-1.6547,-0.0534,0.4389)$ \\
\hline 70 & 0 & 1 & $55,0.0947)$ & $(-0.2508,-0.6555,0.0947)$ \\
\hline 75 & 0 & 1 & $26,0.0052)$ & $(0.0622,-0.0426,0.0052)$ \\
\hline 80 & 0 & 1 & & $(0.1171,0.225,-0.0579)$ \\
\hline 85 & 0 & 1 & $(0.0$ & $(0.0101,-0.3795,0.0038)$ \\
\hline 90 & 0 & 1 & $.1908)$ & $(0.2122,0.6591,-0.1908)$ \\
\hline 95 & 0 & 1 & 104) & $(-0.1686,0.2836,-0.0604)$ \\
\hline 100 & 0 & 1 & $(0.0$ & $(0.0483,0.1285,0.007)$ \\
\hline 105 & 0 & 1 & $(-0.0$ & $(-0.0854,-0.1097,0.0079)$ \\
\hline 110 & 0 & 1 & $(-0.0$ & $(-0.0393,-0.0384,-0.0508)$ \\
\hline 115 & 0 & 1 & $(0.0$ & $(0.0091,0.0336,-0.1149)$ \\
\hline 120 & 0 & 1 & $(-0.5$ & $(-0.5083,0.0726,-0.0915)$ \\
\hline 125 & 0 & 1 & $(0.0$ & $(0.0699,0.0019,0.0156)$ \\
\hline 130 & 0 & 1 & $(-1.2$ & $(-1.299,-0.0268,0.0257)$ \\
\hline 135 & 0 & 1 & $928,0.0246)$ & $(-0.0111,-0.0928,0.0246)$ \\
\hline 140 & 0 & 1 & $(0.0$ & $(0.0347,-0.3627,0.0559)$ \\
\hline 145 & 0 & 1 & $(0.02$ & $(0.0262,-0.3492,-0.0232)$ \\
\hline 150 & 0 & 1 & & $(0.1324,0.287,0.0576)$ \\
\hline 155 & 0 & 1 & $0.1611,0.0153)$ & $(-0.0556,0.1611,0.0153)$ \\
\hline 160 & 0 & 1 & $(0.0145,0.0929,0.0204)$ & $(0.0145,0.0929,0.0204)$ \\
\hline 165 & 0 & 1 & $(0.3499$, & $(0.3499,0.1421,0.0148)$ \\
\hline 170 & 0 & 1 & $(-0.116,0.6657,0.0643)$ & $(-0.116,0.6657,0.0643)$ \\
\hline 175 & 0 & 1 & $(0.0477,-1.3697,0.133)$ & $(0.0477,-1.3697,0.133)$ \\
\hline 180 & 0 & 1 & $(-0.1419,-0.1911,-0.044)$ & $(-0.1419,-0.1911,-0.044)$ \\
\hline 185 & 0 & 1 & $(-0.0558,-0.2044,-0.0153)$ & $(-0.0558,-0.2044,-0.0153)$ \\
\hline 190 & 0 & 1 & $(-0.0864,0.2087,0.027)$ & $(-0.0864,0.2087,0.027)$ \\
\hline
\end{tabular}


Table B-60: Error for Localisation Office Map (UCPF-MCL) Case 3

\begin{tabular}{|c|c|c|c|c|}
\hline$\#$ & $R$ & $\mathrm{C}$ & $r$ & er \\
\hline 5 & & 1 & $0.0088,12.0103,0.0325)$ & $\overline{(-3.1584,-0.8437,-0.9832)}$ \\
\hline 10 & & & $0148,6.3$ & $(0.8596$ \\
\hline 15 & & 1 & $(10.3724,1.4283,2.7882)$ & $(-3.7215,7.9712,1.3418)$ \\
\hline 20 & 1 & 2 & $(-6.8998,17.3046,-4.7399)$ & $(-7.1142,4.9554,-4.7465)$ \\
\hline 25 & & 1 & $(-9.7296,12.2787,-5.095)$ & $(-9.7296,12.2787,-5.095)$ \\
\hline 30 & 2 & 1 & $(-4.9125,32.4943,-5.6957)$ & $(-4.9125,32.4943,-5.6957)$ \\
\hline 35 & 3 & 12 & $(21.8999,34.7917,-1.5413)$ & $(-9.3536,2.0119,-3.0483)$ \\
\hline 40 & 3 & & $(3.0832,-10.3676,0.1298)$ & $(3.0832,-10.3676,0.1298)$ \\
\hline 45 & 4 & & $\overline{(3.8}$ & $(3.85$ \\
\hline 50 & 4 & $J$ & $(-8.0$ & $(-0.42$ \\
\hline 55 & 4 & 4 & $(-0$. & $(-2.1068$ \\
\hline 60 & 4 & 4 & $(-13$ & $(-0.130$ \\
\hline 65 & 4 & 1 & $(-0$. & $(-0.311$ \\
\hline 70 & 4 & 1 & $(-0$ & $(-0.13$ \\
\hline 75 & 4 & 1 & $(-0)$. & $(-0.04$ \\
\hline 80 & 4 & 1 & $(0.0$ & $(0.0238$ \\
\hline 85 & 4 & 1 & $(0.0$ & $(0.089,1.28$ \\
\hline 90 & 4 & 1 & $(0.13$ & $(0.1303,0.7214$, \\
\hline 95 & 4 & 1 & $(0.17$ & $(0.1762,0$. \\
\hline 100 & 4 & 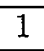 & $\overline{(0.1}$ & $(0.137$ \\
\hline 105 & 4 & 1 & $(-0.0$ & $(-0.0936,-c$ \\
\hline 110 & 4 & 1 & $(-0.0474,-0.0$ & $(-0.0474,-0.0224,0.0132)$ \\
\hline 115 & 4 & 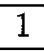 & & $(0.0547,0.0$ \\
\hline 120 & 1 & 2 & & $(-0.4393$ \\
\hline 125 & 1 & 1 & & $(-0.2839$ \\
\hline 130 & 1 & 1 & $(-0.28$ & $(-0.2899,0.1474,0.0072)$ \\
\hline 135 & 4 & 1 & & $(0.0472,-0.04$ \\
\hline 140 & $z$ & & $0.0507)$ & $(0.1311,-0.2824,0.0507)$ \\
\hline 145 & 4 & \pm & $(-0.0111,0.062,0.0402)$ & $(-0.0111,0.062,0.0402)$ \\
\hline 150 & 4 & 1 & $\left(0.4188,0.0^{\prime}\right.$ & $(0.4188,0.0739,0.0908)$ \\
\hline 155 & 4 & 1 & $0.0071)$ & $(-0.0596,0.0496,0.0071)$ \\
\hline 160 & 4 & 1 & $(-0$. & $(-0.033,0.0$ \\
\hline 165 & 4 & 1 & $(0.1$ & $(0.1807,-0$ \\
\hline 170 & 4 & 1 & $(0.1$ & $347,0.0374)$ \\
\hline 175 & 4 & 1 & $0.0449)$ & $(-0.0313,-0.4951,0.0449)$ \\
\hline 180 & 4 & 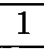 & $(-0.1)$ & $.2147,0.0178)$ \\
\hline 185 & 4 & 1 & & $(-0.2$ \\
\hline 190 & 4 & 1 & $(-0.4772,0.1186,-0.0619)$ & $(-0.4772,0.1186,-0.0619)$ \\
\hline
\end{tabular}


Table B-61: Error for Localisation Office Map (UCPF-MCL) Case 4

\begin{tabular}{|c|c|c|c|c|}
\hline \# & $\mathrm{R}$ & $\mathrm{C}$ & Most Likely Cluster & Closest Cluster \\
\hline 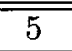 & 0 & 2 & $\overline{(-0.0066,30.7263,0.0397)}$ & $(-1.6482,17.9501,-1.4013)$ \\
\hline 10 & 1 & 1 & $(19.136,15.5443,-0.7573)$ & $(19.136,15.5443,-0.7573)$ \\
\hline 15 & 1 & 2 & $(0.2721,-0.0673,0.017)$ & $(0.2721,-0.0673,0.017)$ \\
\hline 20 & 1 & 1 & $(0.4176,0.0093,0.0198)$ & $(0.4176,0.0093,0.0198)$ \\
\hline 25 & 1 & 1 & $(1.1032,0.0797,0.0211)$ & $(1.1032,0.0797,0.0211)$ \\
\hline 30 & 1 & 1 & $(-0.0738,0.0542,-0.0576)$ & $(-0.0738,0.0542,-0.0576)$ \\
\hline 35 & 1 & 1 & $.0546,-0.0083)$ & $(0.0278,0.0546,-0.0083)$ \\
\hline 40 & 1 & 1 & $56,0.0048)$ & $(0.0638,-0.056,0.0048)$ \\
\hline 45 & 1 & 1 & $0.1259,-0.0171)$ & $(-0.0208,0.1259,-0.0171)$ \\
\hline 50 & 1 & 1 & $(0.2876,0.0122,0.4903)$ & $(0.2876,0.0122,0.4903)$ \\
\hline 55 & 1 & 1 & $2.0074)$ & $(0.3767,-0.023,2.0074)$ \\
\hline 60 & 1 & 1 & $6,-0.017)$ & $(0.2039,0.0486,-0.017)$ \\
\hline 65 & 1 & 1 & $3086)$ & $(0.2142,0.27$ \\
\hline 70 & 1 & 1 & $.0726)$ & $(-0.12,0$ \\
\hline 75 & 1 & 1 & $\left(-0.0^{\prime}\right.$ & $(-0.0781,-0.0219,0.0257)$ \\
\hline 80 & 1 & 1 & $(-0.0464,0.3235,0.0426)$ & $(-0.0464,0.3235,0.0426)$ \\
\hline 85 & 1 & 1 & $(0.00$ & $(0.0062,-0.3926,0.0042)$ \\
\hline 90 & 1 & 1 & $(0.1$ & $(0.195,1.3024,-0.0061)$ \\
\hline 95 & 1 & 1 & $(-0.0$ & $(-0.0607,0.3096,0.0043)$ \\
\hline 100 & 1 & 1 & $(0.0$ & $(0.07$ \\
\hline 105 & 1 & $\overline{1}$ & $(-0.0$ & $-0.0792,0.0034)$ \\
\hline 110 & 1 & 1 & $(-0$ & 5501) \\
\hline 115 & 1 & 1 & $(-0)$. & $(-0.00$ \\
\hline 120 & 1 & $\overline{1}$ & $(-0$. & $(-0.582$ \\
\hline 125 & 1 & 1 & $(-1$. & $(-1.2677,0.0472,0.0132)$ \\
\hline 130 & 1 & 1 & $(-1.2$ & $(-1.3646,-0.033,0.0201)$ \\
\hline 135 & 1 & 1 & $(-0.0$ & $(-0.0182,-0.1076,0.0269)$ \\
\hline 140 & 1 & 1 & $648,0.0537)$ & $(0.0301,-0.3648,0.0537)$ \\
\hline 145 & 1 & 1 & $6,-0.3995,-0.0328)$ & $(0.0096,-0.3995,-0.0328)$ \\
\hline 150 & 1 & 1 & $76,0.0183,0.1207)$ & $(0.1776,0.0183,0.1207)$ \\
\hline 155 & 1 & 1 & $(-0.0489,0.0323,0.0144)$ & $(-0.0489,0.0323,0.0144)$ \\
\hline 160 & 1 & 1 & $(0.0442,0.0949,0.0124)$ & $(0.0442,0.0949,0.0124)$ \\
\hline 165 & 1 & 1 & $(0.3426,0.155,0.0205)$ & $(0.3426,0.155,0.0205)$ \\
\hline 170 & 1 & 1 & $(-0.1138,0.6874,0.0642)$ & $(-0.1138,0.6874,0.0642)$ \\
\hline 175 & 1 & 1 & & $(0.053,-1.3666,0.1329)$ \\
\hline 180 & 1 & 1 & $(-0.1251,0.019,-0.0302)$ & $(-0.1251,0.019,-0.0302)$ \\
\hline 185 & 1 & 1 & $(-0.0218,-0.1$ & $(-0.0218,-0.1981,-0.0073)$ \\
\hline 190 & 1 & 1 & $(-0.0544,0.201,0.0275)$ & $(-0.0544,0.201,0.0275)$ \\
\hline
\end{tabular}


Table B-62: Error for Localisation Office Map (UCPF-MCL) Case 5

\begin{tabular}{|c|c|c|c|c|}
\hline \# & $\mathrm{R}$ & $\mathrm{C}$ & $\mathrm{r}$ & ster \\
\hline$\overline{5}$ & U & 1 & $48,11.908,-0.0089)$ & $\overline{(0.1348,11.908,-0.0089)}$ \\
\hline 10 & 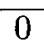 & 2 & $(1.2708,12.545,6.3714)$ & $(1.2708,12.545,6.3714)$ \\
\hline 15 & & 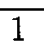 & $.9853,-0.0467)$ & $(16.0953,18.9853,-0.0467)$ \\
\hline 20 & 1 & 8 & $(3.4022,-5.1316,-0.0434)$ & $(0.5725,-0.7969,-0.7379)$ \\
\hline 25 & 1 & 2 & $(8.013,-0.0811,-0.0143)$ & $(2.2046,-5.3208,-0.0818)$ \\
\hline 30 & 1 & 1 & $(10.293,0.3489,-0.0132)$ & $(10.293,0.3489,-0.0132)$ \\
\hline 35 & 2 & 1 & $(21.8032,18.874,-1.5882)$ & $(21.8032,18.874,-1.5882)$ \\
\hline 40 & 2 & 1 & $(20.7067,17.1683,4.3259)$ & $(20.7067,17.1683,4.3259)$ \\
\hline 45 & 3 & 1 & $(3.73$ & $(3.7398,16.4295,-1.2046)$ \\
\hline 50 & 3 & 8 & $(-16.5062,9.4614,-1.4031)$ & $(0.8119,-1.0192,-7.2043)$ \\
\hline 55 & 3 & 1 & $(-21.0203,9.8235,-1.4328)$ & $(-21.0203,9.8235,-1.4328)$ \\
\hline 60 & 3 & 1 & $(-24$. & $(-24.7776,12.0475,4.9583)$ \\
\hline 65 & 4 & 1 & $(-11$ & $(-11.5$ \\
\hline 70 & $\overline{4}$ & 1 & $4,-0.1921)$ & $(0.090$ \\
\hline 75 & 4 & 1 & $(-0.7$ & $(-0.7951,7.8323,-0.3852)$ \\
\hline 80 & 5 & 4 & $11.1,-3.1731)$ & $(-0.0789,3$ \\
\hline 85 & 5 & 4 & $(-0.0$ & $(-0.0147,3$ \\
\hline 90 & 5 & 3 & $(-0.3995$ & $(0.0$ \\
\hline 95 & 5 & 3 & $(0121001$ & $\overline{(0.124}$ \\
\hline 100 & 5 & 1 & & $(0.1062,0.0$ \\
\hline 105 & 5 & 1 & $(-0.1$ & $(-0.1006,-0.078$ \\
\hline 110 & 5 & 1 & $(-0.04$ & $(-0.0437,-1$ \\
\hline 115 & 5 & 1 & & $(0.038,0.6$ \\
\hline 120 & 5 & 1 & $(-0$. & $57,0.0123)$ \\
\hline 125 & $=$ & 1 & & $(-0.2983,0.1568,0.0111)$ \\
\hline 130 & 5 & 1 & & $(-0.2928$ \\
\hline 135 & 5 & 1 & & $(0.0489,-$ \\
\hline 140 & 5 & 1 & $(0$ & $(0.125,-0.2804$ \\
\hline 145 & 5 & 1 & $(-0$. & $(-0.0112,0.0473,0.0359)$ \\
\hline 150 & 5 & 1 & & $(0.4209,0.0742,0.0962)$ \\
\hline 155 & $J$ & 1 & & $(-0.04,0.0528,0.0011)$ \\
\hline 160 & 5 & 1 & $(-0.0$ & $(-0.0311,0.0376,0.0029)$ \\
\hline 165 & 5 & 1 & & $(0.1577,-0.0479,0.0213)$ \\
\hline 170 & $J$ & 1 & & $26,0.0341)$ \\
\hline 175 & $J$ & 1 & $(-0)$. & $(-0.0372,-0.4777,0.0417)$ \\
\hline 180 & 0 & 1 & $(-0.1) \quad-1$ & $(-0.110$ \\
\hline 185 & 0 & 1 & & $(-0.203$ \\
\hline 190 & 5 & 7 & $(-0.477,0.1$ & $(-0.477,0.1026,-0.0563)$ \\
\hline
\end{tabular}


Table B-63: Error for Localisation Office Map (UCPF-MCL) Case 6

\begin{tabular}{|c|c|c|c|c|}
\hline \# & & X & Mos & Closest Cluster \\
\hline$\overline{5}$ & & 1 & $\bar{~} .2223)$ & $\overline{\overline{21.4}}$ \\
\hline 10 & & & 28.9531 & $(28.9531,33.7474,-0.0571)$ \\
\hline 15 & & & $16.0667,18.8523,-0.844$ & $(16.0667,18.8523,-0.8442)$ \\
\hline 20 & & & $(23.8275,-3.0662,-7.0814)$ & $(23.8275,-3.0662,-7.0814)$ \\
\hline 25 & & 1 & $(12.3554,-0.7003,-3.1267)$ & $(12.3554,-0.7003,-3.1267)$ \\
\hline 30 & & 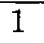 & $(3.5156,-1.7006,-2.9676)$ & $(3.5156,-1.7006,-2.9676)$ \\
\hline 35 & & 1 & $(3.4399$ & 815) \\
\hline 40 & & 2 & $(-20.1$ & ,2.9586) \\
\hline 45 & & 1 & $(4.0$ & $5536)$ \\
\hline 50 & & 7 & $(14.7$ & 191) \\
\hline 55 & & & $(-20$ & $(1.73$ \\
\hline 60 & & & & \\
\hline 65 & & 1 & $(-17$. & $(-17.6)$ \\
\hline 70 & & & $(-0.3)+3)-1$ & \\
\hline 75 & & 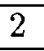 & & $(-0.27$ \\
\hline 80 & & 1 & & \\
\hline 85 & & 1 & & \\
\hline 90 & & 1 & & \\
\hline 95 & & 1 & & $(-0$. \\
\hline 100 & & 1 & & \\
\hline 105 & & 1 & & \\
\hline 110 & & 1 & & $(-0$. \\
\hline 115 & & 1 & $(-0$. & $(-0$. \\
\hline 120 & & 1 & & \\
\hline 125 & & 1 & & $25)$ \\
\hline 130 & & 1 & & 65) \\
\hline 135 & & 1 & & $276)$ \\
\hline 140 & & 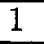 & & \\
\hline 145 & & 1 & & \\
\hline 150 & & 1 & & \\
\hline 155 & & 1 & $(12$. & $7,-5.227)$ \\
\hline 160 & & 1 & (11 & $1,-4.757)$ \\
\hline 165 & & 1 & $(10$. & ,-5.709) \\
\hline 170 & & 1 & $(12$. & ,3.0485) \\
\hline 175 & I & 1 & $(9.9$ & $(9.9143,-12$ \\
\hline 180 & 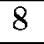 & 1 & $(6.3$ & $\left(6.312^{2}\right.$ \\
\hline 185 & & 1 & $(4.5$ & $(4.5 !$ \\
\hline 190 & 8 & 3 & $(-2.2759,14.2309,-1.7401)$ & $(-2.2759,14.2309,-1.7401)$ \\
\hline
\end{tabular}

